\title{
Nuovi metodi e risultati nella geometria sulle varietà algebriche.
}

\author{
Memoria di Beniamino Segre (a Roma).
}

Sunto. - $\grave{E}$ dato dall' ultimo capoverso dell'Introduzione e dai quattro che lo precedono.

\section{INTRODUZIONE}

Nel suggestivo rapporto presentato al Congresso Internazionale dei Matematici del 1950, lo ZARISKI [1] (1) dà giusto rilievo ai bei risultati ottenuti da lui e dalla sua Scuola negli ultimi 25 anni, col proseguire ed estendere sistematicamente quel processo di aritmetizzazione della geometria algebrica i cui primordi risalgono a Dedekind e Weber. In tale rapporto, mentre si riconosce ai geometri italiani il merito di aver eretto - sia pure, in una prima fase, su basi non del tutto compinte - il magnifico edificio della teoria delle superficie, si afferma in pari tempo che - agli albori del nostro secolo - la geometria algebrica era giunta ad un punto morto, si che il processo di aritmetizzazione si presentava come l'unica via atta a porne $i$ fondamenti su basi sicure ed a preparare la costruzione della teoria delle varietà superiori.

Ora, se anche ̀̀ vero che un' esposizione concisa delle questioni di geometria algebrica fatta coi metodi algebricc-geometrici - che hanno generalmente carattere più sintetico - può dare talora l'impressione di difetto di rigore, pare tuttavia che $i$ suddetti apprezzamenti non tengano nel debito conto l'ampio lavoro di consolidamento dei fondamenti della geometria algebrica nell' indirizzo classico compiuto dal Severi nell' ultimo ventennio, e dimentichino del tutto numerosi ed importanti contributi costruttivi in tale indirizzo alla teoria delle varieta superiori occorsi nell'ultimo cinquantennio. Basti citare in proposito l'estensione alle varieta dell'invariante di Zeuthen (C. Segre, Alexander), l'irregolarità superficiale delle varietà (Castelnuovo ed Enriques), un complesso di essenziali proprietà fondamentali di geometria sulle varietà (Severi), la teoria delle varietà abeliane e quasi abeliane (Lefschetz, Scorza, Rosati, Conforto, Severi), le proprietà di unirazionalità e razionalità di varietà superiori e le applicazioni all'analisi diofantea (Enriques, Fano, Roth, B. Segre), la teoria delle varieta grassmanniane (Severi, Hodge). Ma sopratutto occorre ricordare il profondo rinnovamento apportato all'indirizzo classico dalla

(1) I numeri entro parentesi quadre rinviano alla Bibliografia posta alla fine del presente lavoro. 
teoria delle serie e dei sistemi d'equivalenza, nella quale sono dominanti i concetti di varietà e d'intersezioni virtuali, con l'introduzione del relativo simbolo d'intersezione. A questa teoria, creata dal Severi a partire dal 1932, hanno fra gli altri contribuito Enriques, Comessatti, B. Segre, J. A. Todd ed Eger $\left({ }^{2}\right)$ : ed appunto alla teoria medesima d dedicato il presente lavoro.

Uno dei risultati salienti in essa già acquisiti è l'esistenza - sopra una qualunque $V_{k}$ algebrica (irriducibile e non singolare) - di $k$ sistemi di equivalenza, invarianti di fronte alle trasformazioni birazionali regolari di $V_{k}$; tali sistemi furono chiamati i sistemi canonici di $V_{k}$, e constano di varietà (effettive o virtnali) - in generale fra loro indipendenti rispettivamente di dimensione $0,1, \ldots, k-1\left(^{3}\right)$. Intersecando fra loro varieta canoniche di dimensioni opportune si ottengono su $V_{k}$ gruppi di punti appartenenti a serie di equivalenza invarianti, l'ordine delle quali fornisce dunque degli invarianti birazionali di $V_{k}$, collegati in parte alla teoria delle forme differenziali di $1^{a}$ specie attaccate a $V_{k}$. Nè mancano profondi legami fra le varietà canoniche e gli integrali armonici su $V_{k}$, come risulta da recenti lavori di Chens [1] e di Hodge [1].

Altre notevoli applicazioni delle nuove teorie geometriche sono quelle concernenti i problemi d'intersezione non regolare fra varietà algebriche, nell'ipotesi cioè che dell'intersezione faccia parte qualche componente di dimensione maggiore del normale. Si tratta di problemi non facili, ai quali il SEverI aveva dedicato nel 1902 un classico lavoro [1] coi metodi della geometria numerativa, ma a cui per oltre un trentennio non fu arrecato nessun contributo ulteriore degno di nota; ed è soltanto a partire dal 1934 che, poggiando sulla teoria delle serie e dei sistemi d'equivalenza, quei pro. blemi furono ripresi ed approfonditi, specialmente nel caso di una varietat ambiente a tre o quattro dimensioni ( $\left.{ }^{4}\right)$.

(2) Cfr. i lavori dei suddetti autori citati nella Bibliografia, ed inoltre l'esposizione d'assieme di SEVERI [6] ed il rapporto di Conforto [1], ove trovansi anche numerose indi. cazioni bibliografiche uiteriori.

(3) Le $V_{k-1}$ canoniche di $V_{k}$ si definiscono e si presentano come estensioni di quanto fu fatto da Riemann e Clebsch per $k=1$ e da Noether ed Enriques per $k=2$. Il primo caso essenzialmente nuọvo è quello della serie di equivalenza canonica sulle superficie, acquisita dal Severi [3,4], e che l'Enriquas [1] ha percio suggerito di chỉamare la serie di Severi. I sistemi canonici delle varie dimensioni furono poi introdotti sulle varieta tridimensionali da B. SEGRE [1] e successivamente sulle varietà superiori da EGER [2-3] e J. A. Topd [2, 7, 8].

(4) Cfr. B. Segre [1, 3], Severi [10], J. A. Tond [5, 8], Archpold [1], Barker [1]. Il Todd, nel primo dei due suoi lavori testè citati, riferendosi ai contributi precedenti sull' argomento così si esprime:

-Segre's work represents an advance on that of his predecessors in three respects. - First, it applies to intersections on a general $V_{3}$, whereas previous results had referred " only to intersections in ordinary space $S_{3}$. Secondly, the results are presented in a form - which is birationally invariant, whereas previous results were of a purely projective cha. - racter. And finally, while the previous results were purely enumerative, Segre's results - are stated in the form of relations of equivalence between eurves or sets of point on $V_{3}$. 
Nonostante l'importanza dei risultati finora conseguiti nei suddetti argomenti, si era ben lungi dal possedere una teoria generale delle intersezioni non regolari : quanto già si conosceva al riguardo, poteva anzi lasciar presumere che, per fondare una siffatta teoria, si avessero a dover superare difficoltà gravissime, sia concettuali che formali. E neppure la teoria delle varietà canoniche poteva dirsi sistemata in modo del tutto soddisfacente, si che ad esempio il Hodge - nel lavoro citato - osserva che «the only papers which 》 deal with canonical systems treat them in broad outline, and many points $\gg$ of detail have still to be investigated, with the result that there are very "few properties of canonical systems which can be used".

La presente Memoria, non solo arreca numerosi nuovi contributi a quelle teorie, ma introduce nello studio di esse nuovi metodi - sia concettuali che formali - ohe ne cambiano radicalmente l'aspetto. Tali metodi, pur avendo le loro radici nell'intuizione geometrica, sono formalmente algebrici e concettualmente topologici, e potranno presum: Imente venir applicati a casi molto più generali, in guisa p. es. da trasportare dette teorie alle varietà topologiche differenziabili (il che permetterebbe di gingere alle classi di omologia di Stiefel-Whitney $e$ di Pontrjagin in modo assai più semplice di come oggi non si sappia fare con la teoria delle ostruzioni, e di ottenere per quelle un vasto assieme di proprietà nuove) Per ragioni di spazio, qui non ci occuperemo però di siffatte estensioni $\left(^{5}\right)$; ed ometteremo altresi lo studio dal punto di vista assiomatico di certe nuove strutture algebriche che qui si presentano, che pure parrebbe degno d'interesse.

Allo studio algebrico-geometrico di dette strutture, è, dedicato il primo dei sette Capitoli in cui il lavoro è diviso. Tali strutture fanno intervenire opportune nozioni gruppali $\left({ }^{6}\right)$, e consistono di un' infinita di anelli (gli anelli d'equivalenza di $V_{k} \theta$ delle varietà contenute in $V_{k}$ ), i cui elementi sono convenientemente fra loro collegati mediante operazioni di vari tipi (fra cui taluno del tutto nuovo) e parzialmente ordinati a mezzo della relazione d'inclusione. Accanto all'introduzione di tali operazioni, va segna. lata la considerazione di certe successioni di varieta e l'estensione a queste ultime di un utile formalismo algebrico.

$\mathrm{Nel}$ Capitolo $2^{\circ}$, poggiando sulle precedenti nozioni, perveniamo ad associare ad ogni varietà $M_{h}^{\prime}$ di $V_{k}$ una successione covariante d'im. mersione di sostegno $M_{h}$, ossia $h$ varietà $M_{h-1}, M_{n-2}, \ldots, M_{0}$ definite intrinsecamente su $M_{h}$ a meno di un'equivalenza (e riducentisi alle successive

(5) Estensioni che ho proposte ad un mio discepolo, e per le quali si dovxà tener conto della teoria dell'omologia su di una varieta topologica relativa, dovata a Lefschetz (cfr. p. es. Lefschetz [1] p. 35 o [2] p. 209).

(6) L'opportunità d'introdurre nozioni gruppali nello studio dell'equivalenza fra varietà algebriche trovasi in J. A. TODD [1] e StrERI [7]. La considerazione esplicita dell' an ello di equivalenza deresi a $J$. A. TODD [8], il quale $\nabla i$ è pervenuto poggiando sulle nozioni di varieta ed intersezioni virtuali secondo Severi, nonchè sul fatto - pure acquisito in SEvert [5] - che l'equivalenza sopra una sottovarieta di $V_{k}$ implica l'equivalenza su $V_{k}$. 
varietà caratteristiche del sistema lineare $\left|M_{h}\right|$, prese con segni opportuni nel caso particolare in cui $M_{h}$ sia un'ipersuperficie di $V_{k}$ ). Varietà siffatte già erano state costruite da ToDD [8] con l'uso delle varietà canoniche di $M_{h}$ e di $V_{k}$; e caratteri numerici d'immersione, di una varietà in un'altra, erano stati considerati prima ancora dal SEVERI [1]. Qui però raggiungiamo lo scopo in modo semplice e diretto, poggiante essenzialmente sulla considerazione di certe intersezioni non regolari, sì da ottenere in pari tempo con relativa facilità varie proprietà e relazioni funzionali notevoli concennenti quelle successioni covarianti. Mostriamo pure (n. 16') come alle suddette successioni covarianti si possa pervenire anche più rapidamente con mezzi di carattere topologico, dopo aver applicato a $V_{k}$ una dilatazione di base $M_{h}$.

L' utilità e, per cosi dire, la spontaneità dei concetti e dei metodi suacennati si palesa appieno nei due Capitoli successivi, dedicati rispettivamente alla teoria generale delle intersezioni non regolari, ed allo studio di varie altre questioni funzionali in cui intervengono varieta do. tate di punti multipli. E persino maggiore si dimostra la portata delle nozioni precedenti nel Capitolo sesto, ove le varieta canoniche di $V_{k}$ si ottengono considerando la successione covariante d'immersione della varietà diagonale del prodotto diretto $V_{k} \times V_{k}$ entro il prodotto medesimo. La nnova definizione che cosl si ottiene per le varieta canoniche $\left({ }^{7}\right)$ è enormemente più semplice di quelle note fino ad oggi, poggianti su intricate considerazioni concernenti le varietà jacobiane di certi sistemi lineari d'ipersuperficie, e - a differenza di queste - ha carattere essenzialmente topolo. gico. Essa permette di stabilire rapidamente ed in modo suggestivo tutto un insieme di proprietà nuove delle varietà canoniche, e di ottenere $i$ legami che intercorrono fra le varietà covarianti d'immersione e le varietà canoniche. All' uopo vengono fra l'altro adoperati alcuni risultati - per sè non privi d'interesse - concernenti i prodotti diretti di due o più varietà algebriche, ai quali appunto $\partial$ dedicato il precedente Capitolo $5^{\circ}$.

L' ultimo capitolo stabilisce i necessari raffronti fra la nuova e le vecchie definizioni delle varietà canoniche $\left({ }^{8}\right)$, ed espone un certo numero di esempi scelti in modo da istituire collegamenti espliciti fra le teorie qui esposte e qualche risultato anteriormente noto. Non insistiamo però granchè a tale riguardo per ragioni di spazio: in particolare, tasciamo per lo più al Lettore il non difficile compito di dedurre dai nostri risultati funzionali corrispondenti relazioni di carattere numerativo, ritrovando cosi fra l'altro - sovente sotto forma più estesa - varie formule di SEvERI $[1,2,9]$, Albanese [1], J. A. Todd [3], Todd e Maxwell [1]. Per lo stesso motivo,

(7) Per la quale vi era soltanto un precedente, dovuto a Comessatri [1], nel caso estremamente particolare della serie d'equivalenza di Severi : efr. quanto è detto al riguardo più oltre, in nota al n. 66 .

(8) Ciò porge, fra l'altro, il significato funzionale della nota definizione topologica di Alexander per l'invariante di Zeuthen-Segre di una $V_{k}$ algebrica (n. 90). 
vien qui omesso d'indagare il comportamento degli enti ottenuti su $V_{h}$ di fronte alle trasformazioni birazionali non regolari di $V_{k}$ (dotate cioè di elementi fondamentali); per uno studio parziale di tale questione, rinviamo a B. Stgre [3] ed a J. A. Todd $[4,6]$.

Un elenco abbastanza particolareggiato dei diversi argomenti trattati nel presente lavoro vien fornito dall'Indice, posto alla fine; cfr. altresì le due Note riassuntive di B. SEGre [8,9]. Aggiungasi che buona parte dei risultati qui conseguiti erano già fin dal 1939 in possesso dell'antore, che l' 8 novembre 1939 ne fece oggetto di una conferenza presso l'Università di Cambridge.

\section{CAPITOLO PRIMO}

\section{PRELIMINARI}

\section{§ I. - L'anello d'equivalenza $\mathfrak{Z}_{V}$ sopra una varietà algebrica $V$.}

1. Nel presente lavoro tratteremo varie questioni di geometria sopra una varietà algebrica $V$, nel campo complesso; la. $V$ sarà sempre supposta irri. ducibile, di dimensione $v \geq 1$ e non singolare (ossia priva di punti multi. pli). Le sottovarieta algebriche di $V$ verranno denotate con lettere latine maiuscole; useremo i caratteri tondi nel caso generale di varieta pure od impure, ed i caratteri ordinari $M, N, P, \ldots$ (eventualmente muniti di indici inferiori o superiori) per designare varieta pure, riservando le prime lettere $A, B, C, \ldots$ dell'alfabeto per le ipersuperficie (ossia per le varietà pure di dimensione $v-1)$ di $V$. Le dimensioni delle varietà pure $M$, $N, P, \ldots$ e gli ordini delle ipersuperficie $A, B, C, \ldots$ saranno denotati con le rispettive lettere latine minuscole $m, n, p, \ldots$ ed $a, b, c, \ldots$.

Una qualunque varietà $\mathscr{N}$ effettiva contenuta in $V$ definisce univocamente le proprie componenti irriducibili (in numero finito), ciascuna $M^{*}$ delle quali da contarsi in essa con una certa molteplicita $\mu_{i}>0$. Cio si esprime con l'uguaglianza

$$
\mathfrak{N C}=\Sigma \mu_{i} M^{i} ;
$$

e si noti che non resta escluso che una delle $M^{i}$ coincida con la $V$, e che di due diverse $M^{i}$ una contenga l'altra. L'insieme delle sottovarietà effettive di $V$ risulta chiuso rispetto all'operazione di somma, e costituisee quindi un semigruppo abeliano. Da esso si deduce un gruppo abeliano, che denoteremo con $\Gamma_{V}$, con l'ampliarlo mediante l'aggiunzione delle varietà virtuali, differenze fra due varietà effettive di $V$; gli elementi $9 R$ di $\Gamma_{V}$ si rappresentano ancora mediante somme finite del tipo (1), ove attualmente i coefficienti $\mu_{i}$ possono assumere valori interi $(0)$ qualsiansi, e fra essi si opera per somma e sottrazione in modo del tatto ovvio. In particolare, lo zero di $T_{V}$ si designerà - secondo l'uso - col simbolo 0 e si chiamerà la varietà nulla di $V$. 
Il gruppo $\Gamma_{V}$ risulta la somma diretta di $v+1$ gruppi abeliani, ciaseuno $\Gamma_{V}^{i}$ dei quali consta degli elementi puri di data dimensione $i$ $(=0,1, \ldots, v)$; in particolare $\Gamma_{V}^{v}$ ̀े il gruppo ciclico infinito formato dai multipli interi (positivi, nulli o negativi) di $V$. Corrispondentemente, ogni sottovarietà $\mathscr{T}$ di $V$ può scriversi in uno ed in un sol modo nella forma

$$
\text { ⿰R丨 }=M^{0}+M^{1}+\ldots+M^{v} \text {, }
$$

dove $M^{i}$ denota una varietà pura (eventualmente nulla) di dimensione $i$.

2. Preso un qualunque intero $m$ soddisfacente alle limitazioni $0 \leq m \leq v-1$, consideriamo su $V v-m$ sistemi lineari d'ipersuperficie effettive

$$
\left|A^{1}\right|,\left|A^{2}\right|, \ldots,\left|A^{v-m}\right|
$$

situati in posizione generica. Con ciò intendiamo significare che, fuori di eventuali varietà fisse o semifisse (appartenenti cioè alle basi di quei sistemi), $v-m$ ipersuperficie tratte in modo generico rispettivamente dai sistemi (1) debbano segarsi semplicemente secondo una varietà pura, $M$, di dimensione $m$. Un insieme di varieta $M$ effettive ottenibili in tal guisa, completato con i suoi elementi di accumularione, è ciò. che chiamasi un sistema elementare effettivo; e la nozione si estende subito ai sistemi elemen. tari virtuali, assumendo virtuali i sistemi lineari d'ipersuperficie (1).

Si dimostra, col Severi [6, nn. 40-48], che la totalità dei sistemi elementari virtuali (ma non quella dei-sistemi elementari effettivi) risulta chiusa di fronte alle operazioni di somma e di differenza. Ciò val quanto dire che le varietà pure, di dimensione $m$, esprimibili quali differenze di due varietà di uno stesso sistema elementare costituiscono un gruppo abeliano, $\Omega_{V}^{m}$, sottogruppo di $\Gamma_{V}^{m}$. Ogni varietà pura $M$ di $V$ appartiene ad una ed una sola classe laterale di $\Omega_{V}^{m}$ in $\Gamma_{V}^{m}$, data da $M+\Omega_{V}^{m}$; tale classe chiamasi il sistema d'equivalenza definito da $M$. Per non moltiplicare troppo le notazioni, noi designeremo una di quelle classi con la stessa lettera $M$ che denota un suo rappresentante; quando ciò si prestasse ad ambiguità, un particolare rappresentante della classe $M$ verrà indicato col simbolo $\langle M\rangle$. Con tali convenzioni, un' uguaglianza fra classi

$$
M=N
$$

si traduce nel fatto che un qualunque rappresentante $\langle M\rangle$ dell' una ed un qualunque rappresentante $\langle N\rangle$ dell'altra appartengono allo stesso sistema d'equivalenza, ossia sono - come si suol dire - fra loro equivalenti su $V$; in tal caso - secondo l'uso - scriveremo che è

$$
\langle M\rangle \equiv\langle N\rangle
$$

(su V),

e ne seguirà l'. nguaglianza $m=n$ fra le relative dimensioni. Qualora i rappresentanti delle classi $M$ ed $N$ si designino con queste stesse lettere, la (2) non esprime naturalmente che $\mathrm{i}$ due rappresentanti debbano coincidere, ma 
soltanto che è

$$
M \equiv N
$$

(su V);

per significare che tali rappresentanti coincidono, introdurremo il segno $\doteq$ e scriveremo che è

$$
M \doteq N
$$

I sistemi d'equivalenza di varietà di data dimensione $m$, composti per somma, costituiscono un gruppo abeliano $\mathcal{C}_{V}^{m}$, gruppo quoziente di $\Gamma_{V}^{m}$ ed $\mathbf{Q}_{V}^{m}$. Quale somma diretta dei gruppi $\mathfrak{C}_{V}^{0}, \mathfrak{C}_{V}^{1}, \ldots, \mathfrak{C}_{V}^{v-1}$ e $\Gamma_{V}^{v}$ si ottiene un gruppo abeliano, che chiameremo il modulo d'equivalenza di $V$ e denoteremo con $\mathfrak{C}_{V}$; esso può anche ottenersi come gruppo quoziente di $\Gamma_{V}$ e della somma diretta $\Omega_{V}^{0}+\Omega_{V}^{1}+\ldots+\Omega_{V}^{v-1}$. Elementi di $\mathcal{C}_{V}$ sono i sistemi generali d'equivalenza, ciascuno dei quali risulta univocamente definito da un suo elemento arbitrario, o rappresentante, sottovarietà (effettiva o virtuale, pura od impura) di $V$; un tal sistema verrà denotato con la stessa lettera $\mathscr{K}$ che designa un suo rappresentante, specificando quest' ultimo con (TK) quando occorra. Ogni sistema d'equivalenza $\mathscr{K}$ si decompone univocamente in una somma di sistemi d' equivalenza puri, mediante la formula $\left(2_{1}\right)\left({ }^{9}\right)$.

Osservazions $1^{\text {a }}$. - Le considerazioni precedenti potrebbero venir estese al caso in cui $V$ sia riducibile, generalizzando ciò che è stato fatto dal Severi [6, cap. V] per le curve riducibili. Inoltre la definizione dei gruppi abeliani $\Gamma_{V}^{m}, \mathbf{Q}_{V}^{m}, \mathfrak{C}_{V}^{m}$ si applica senza modificazioni ad una varietà irriducibile $V$ che sia singolare, nell'ipotesi che il luogo dei punti multipli di $\checkmark$ abbia dimensione inferiore ad $m$.

OSSERVAzIONe 2a - Accanto all'equivalenza dianzi considerata, e che SEVERI ha chiamato l'equivalenza razionale, sono stati introdotti altri tipi d'equivalenza, detti l'equivalenza algebrica e l'equivalenza aritmetica (cfr. SEVERi [7]). Tutti gli sviluppi del presente lavoro potrebbero - con lievi modificazioni venir trasportati a queste equivalenze; basta però limitarsi, come noi faremo, al caso dell' equivalenza razionale, ponendosi nel quale i nostri risultati assumono la massima significativita funzionale.

3. Se $M$ ed $N$ sono due varietà pure effettive di $V$ (di dimensioni $m$ ed $n$ ), designeremo con $(M N\rangle$ la loro interferenza; è noto che questa è una varietà algebrica, ogni componente della quale ha dimensione non inferiore ad $m+n-v$, e che - in generale, e nell'ipotesi che sia $m+n-v \geq 0-$ cia. scuna componente di $\langle M N\rangle$ ha di fatto la dimensione $m+n-v$. Diremo che $M$ ed $N$ sono in posizione regolare entro $V$, se valgono le seguenti condizioni:

(9) Qui ed in seguito, l'indice in basso sta per indicare che ci si riferisce ad una for. mula di un numero precedente, $\left(2_{4}\right)$ denotando precisamente la formula (2) del n. 1 . Si noti però che, attualmente, la $\left(2_{1}\right)$ viene ad assumere significato un po' diverso da quello che avera la (2) nel n. 1, esprimendo ora un legame fra sistemi d'equivalenza (anzichè fra curve). 
B. SFGRE: Nuovi metodi, e risultati nella geometria, esc.

a) l'interferenza $\langle M N\rangle$ ̀̀ una varietà pura $P$, avente dimensione regolare (1)

$$
p=m+n-v
$$

con l'intesa che $p<0$ implichi che $M$ ed $N$ non abbiano punti a comune, e ciò̀ che risulti $P \doteq 0$.

b) se $p \geq 0$, le $M, N$ si segano semplicemente lungo $P$. Con cio intendiamo significare, che, preso un punto $O$ generico di $P$ (e cioe di una qua. lunque componente di $P$ ), non solo le $V, P$, ma anche le $M, N$, debbano passare semplicemente per $O$; inoltre, detti rispettivamente $O_{V}, O_{P}, O_{M}, O_{N}$ gli spazi lineari tangenti in $O$ alle $V, P, M, N$, debba $O_{V}$ risultare lo spazio congiungente di $O_{M}, O_{N}$, il che - a norma della (1) - val quanto dire che $O_{M}$ ed $O_{N}$ debbano segarsi secondo $O_{P}$.

La nozione di varietà in posizione regolare entro $V$, si estende subito ad un qualunque numero di sottovarieta $d i$

Nelle ipotesi suddette, e tenuto conto del n. 2, si vede che, se le varietà $M$ ed $N$ variano ciascuna in un sistema elementare effettivo, lo stesso puo dirsi della $P$. Il risultató si estende nel modo seguente al caso di varietà pure $M, N$ variabili entro sistemi d'equivalenza. Si ponga (com'è sempre possibile)

$$
M=\Sigma M_{i}-\Sigma M_{i^{\prime}}^{\prime}, \quad N=\Sigma N_{j}-\Sigma N_{j^{\prime}}^{\prime},
$$

dove le varietà $M_{i}, M_{i^{\prime}}^{\prime}, N_{j}, N_{j^{\prime}}^{\prime}$ varino entro sistemi elementari effettivi, in guisa che per ciascuna coppia $\left(M_{i}, N_{j}\right),\left(M^{\prime}{ }^{\prime}, N_{j}\right),\left(M_{i}, N^{\prime}{ }^{\prime}\right),\left(M^{\prime}{ }_{i}, N^{\prime}{ }^{\prime}\right)$ valgano le suddette condizioni $a$ ) e b). Si vede allora che, comunque si scelgano tali varieta, la

$$
P \doteq \Sigma\left\langle M_{i} N_{j}\right\rangle-\Sigma\left(M^{\prime}{ }_{i} N_{j}\right\rangle-\Sigma\left\langle M_{i} N^{\prime}{ }_{j^{\prime}}\right\rangle+\Sigma\left\langle M_{i^{\prime}}{ }^{\prime} N^{\prime}{ }_{j^{\prime}}\right\rangle
$$

varia entro un sistema d'equivalenza. La varietà $P$ chiamasi l'intersezione virtuale delle $M, N$; la sua dimensione $p$ è data dalla (1), e $p<0$ implica ch'essa si annulli. Qualora $M, N$ e $\boldsymbol{P}$ si pensino quali elementi del modulo d'equi. valenza $\mathcal{C}_{V}$ di $V$, si potrà considerare $P$ come prodotto di $M$ ed $N$, e scrivere

od anche semplicemente

$$
P=(M N)_{V},
$$

$$
P=M N
$$

quando non vi sia possibiliti d' equivoco.

La definizione del prodotto si estende subito ad elementi

$$
\mathfrak{T}=\Sigma M^{i}, \quad \mathscr{T}=\Sigma N^{j}
$$

arbitrari di $\mathfrak{C}_{V}$, assumendo

$$
\mathscr{\mathfrak { T }}=\Sigma M^{i} N^{j} .
$$

Si constata senza difficolta che tale prodotto risulta commutativo, associativo e distributivo rispetto alla somma, sicchè $\mathfrak{C}_{V}$ acquista mediante esso la struttura di un anello commutativo graduato. Denoteremo con $\mathfrak{E}_{V}$ questo 
anello, e lo chiameremo l'anello d' equivalenza di $V$ (cfr. ToDD [1], [7] § 2); esso è dotato di unità, l'unità di $\mathfrak{z}_{V}$ essendo manifestamente $V$. L'elevazione a potenza (con esponente intero positivo o nullo) di un elemento di $\mathfrak{A}_{V}$, verrà indicata scrivendo l'esponente in alto a destra entro parentesi quadre (onde evitare ch'esso possa confondersi con un indice superiore).

4. Prese su $V$ una qualunque sottovarietà (effettiva o virtuale) $M$ ed una sottovarieta effettiva $V^{\prime}$ (di dimensione $v^{\prime}<v$ ), diremo che $M$ appartiene a (od è contenuta in) $V^{\prime}$ e che $V^{\prime}$ contiene $M$, e scriveremo che è

$$
\left.M \subset V^{\prime} \quad \text { (od anche } V^{\prime} \supset M\right),
$$

se esistono due sottovarieta effettive $M_{1}, M_{2}$ di $V^{\prime}$ (eventualmente costituite da multipli di $V^{\prime}$ ) tali che si abbia

$$
M_{1}-M_{2} \equiv M
$$

(su V).

E si noti che, scrivendo la (1), oltre a quest' ultima condizione intendiamo significare che la varietà $V^{\prime}$ debba essere effettiva. $\dot{E}$ subito visto che la relazione espressa dal simbolo $\subset$ ha carattere transitivo; inoltre, qualunque siano le sottovarietà $N$ e $V^{\prime}$ di $V$, risulta

e dalla (1) seguono le:

$$
\left(N V^{\prime}\right)_{r} \subset V^{\prime}
$$

$$
(M N)_{V} \subset\left(N V^{\prime}\right)_{V} \subset V^{\prime}
$$

Si ha poi che, se $V^{\prime} \dot{\theta}$ una sottovarietà irriducibile di $V$, che sia non singolare od almeno tale che il luogo dei suoi panti multipli abbia dimen* sione inferiore ad $m$, e se $M, N$ denotano due sottovarietà di $V^{\prime}$, dalla

segue la

$$
M \equiv N
$$

$\left(s u V^{\prime}\right)$

$$
M \equiv N
$$

$(s u \quad V)$

ma non necessariamente viceversa. La prima parte di questa proposizione si prova agevolmente tenuto conto del $n$. 2, stabilendola dapprima nell'ipotesi che $M$ ed $N$ appartengano ad un medesimo sistema elementare effettivo, e passando poi al caso generale. La seconda parte si stabilisce senza difficoltà con esempi (cfr. SEvERI [6], n. 45).

Nelle suddette ipotesi per $V^{\prime}$, ogni classe d'equivalenza $M$ di $V^{\prime}$ definisce quindi una classe d' equivalenza di $V$, che si potrà ancora indicare col simbolo $M$, soddisfacente alla (1). E subito visto che si ottiene cosi un omomorfismo di $\mathfrak{C}_{V^{\prime}}^{m}$ in $\mathfrak{C}_{V}^{m}$, e precisamente un omomorfismo di $\mathfrak{C}_{V}^{m}, s u l$ sottomodulo degli elementi $M d i \mathfrak{C}_{V}^{m}$ che soddisfano alla (1). Se $\nabla^{\prime}$ è non singolare tutto ciò vale per $m=0,1, \ldots, v^{\prime}$, e dà luogo ad un omomorfismo del modulo d'equivalenza $\mathfrak{C}_{V}$, di $V^{\prime}$ nell' analogo modulo d' equivalenza $\mathfrak{C}_{V}$ di $V$; 
questo, però, non si estende ad un omomorfismo fra gli anelli di equivalenza $\mathfrak{Z}_{V^{\prime}}, \mathfrak{z}_{V}$, come si ha ovviamente notando che le varietà $(M N)_{V^{\prime}}$ ed $(M N)_{V}$, nell'ipotesi che siano entrambe definite e non nulle, risultano di dimensioni diverse (rispettivamente uguali ad $m+n-v^{\prime}$ e $m+n-v$ ).

5. Ci proponiamo ora di stabilire alcune proposizioni relative agli anelli $d i V$, leganti ciod fra loro l'anello d'equivalenza di $V$ e gli anelli d'equiva. lenza delle sottovarieta di $V$. Supporremo sempre che ciascuna delle sottovarietà che cosl intervengono sia effettiva e non singolare, od almeno che sia dotata soltanto di singolarità di dimensione inferiore a quella delle classi d'equivalenza che su essa si considerano.

a). - Se $M, N, \ldots, P, R, V^{\prime}$ appartengono a $V$ e sussistono le$$
R \subset V^{\prime} \subset V \text {. }
$$

posto per abbreviare

risulta

$$
M^{\prime}=\left(M V^{\prime}\right)_{V}, N^{\prime}=\left(N V^{\prime}\right)_{V}, \ldots, P^{\prime}=\left(P V^{\prime}\right)_{V}
$$

$$
(R M N \ldots P)_{V}=\left(R M^{\prime} N^{\prime} \ldots P^{\prime}\right)_{V^{\prime}} .
$$

Supposto anzitutto che ciascuna delle varietà che compaiono nelle (1), (2), (3) sia effettiva e non nulla, in virtù delle (2) fra le relative dimensioni intercedono le relazioni

$$
m-v=m^{\prime}-v^{\prime}, n-v=n^{\prime}-v^{\prime}, \ldots, p-v=p^{\prime}-v^{\prime}
$$

sicohè i due membri della (3) hanno la stessa dimensione virtuale

(4) $r+(m-v)+(n-v)+\ldots+(p-v)=r+\left(m^{\prime}-v^{\prime}\right)+\left(n^{\prime}-v^{\prime}\right)+\ldots+\left(p^{\prime}-v^{\prime}\right)$.

Se inoltre, entro $V$, la $V^{\prime}$ risulta in posizione regolare rispetto a ciascuna delle $M, N, \ldots, P$, si pud assumere (n. 3$)$ :

$$
M^{\prime} \doteq\left\langle M V^{\prime}\right\rangle, N^{\prime} \doteq\left\langle N V^{\prime}\right\rangle, \ldots, P^{\prime} \doteq\left\langle P V^{\prime}\right\rangle
$$

allora, in base alle (1), il luogo dei punti comuni alle $R, M, N, \ldots, P$ coincide col luogo dei punti comuni alle $R, M^{\prime}, N^{\prime}, \ldots, P^{\prime}$, e si ha quindi

$$
\langle R M N \ldots P\rangle \doteq\left\langle R M^{\prime} N^{\prime} \ldots P^{\prime}\right\rangle \text {. }
$$

Supponiamo infine ulteriormente che le varieta $R, M, N, \ldots, P$ risultino fra loro in posizione regolare entro $V$, e cioè (n. 3) che $(R M N \ldots P)$ sia una varietà pura, avente la dimensione regolare (4), e che lungo essa le $R, M, N, \ldots, P$ si seghino semplicemente. Allora un generico punto $O$ della (6) è semplice per ciascuna delle varietà $R, M, N, \ldots, P$, ed i relativi spazi tangenti $O_{n}$, $O_{M}, O_{N}, \ldots, O_{P}$ si segano secondo lo spazio $O_{6}$ tangente in $O$ alla (6). In virtù di quanto si è ammesso al principio di questo numero, il punto $O$ risulta semplice anche per $V^{\prime}$, e sia $O_{V}$, il relativo spazio tangente. Questo, 
a norma delle (1) e delle ipotesi fatte sulle (5), conterra $O_{R}$ - e quindi pure $O_{6}$ - e segherà $O_{R}, O_{M}, O_{N}, \ldots, O_{P}$ rispettivamente secondo gli spazi $O_{R}$, $O_{M^{\prime}}, O_{N^{\prime}}, \ldots, O_{P^{\prime}}$ tangenti in $O$ alle $R, M^{\prime}, N^{\prime}, \ldots, P^{\prime} ;$ ne consegue che l'intersezione di questi ultimi spazi tangenti coincide col suddetto $O_{6}$, sicchè le $R, M^{\prime}, N^{\prime}, \ldots, P^{\prime}$ si segano semplicemente lungo la (6) e sono quindi in posizione regolare entro $V^{\prime}$. Ma allora, nelle ipotesi ammesse, dalla (6) si deduce appunto la relazione (3).

Il ragionamento precedente mostra pure che, se una delle varietà $M$, $N, \ldots, P, M^{\prime}, N^{\prime}, \ldots, P^{\prime}$ si annulla, allora ciascnno dei due membri della (3) anche si annulla, sicchè la (3) continua a sussistere.

Dal caso dianzi trattato, di varietà $M, N, \ldots, P, M^{\prime}, N^{\prime}, \ldots, P^{\prime}$ effettive e soggette ad opportune condizioni di genericità, si passa poi agevolmente al caso più generale, notando ohe sia l'uno ohe l'altro dei due membri della (3) dipende linearmente ed omogeneamente da ciascuna delle $M$, $N, \ldots, P$. D'altro canto, si può in ogni caso assumere

$$
M=M_{1}-M_{2}, N=N_{1}-N_{2}, \ldots, P=P_{1}-P_{2},
$$

facendo in modo che le condizioni suddette abbiano a valere qualora, per ogni scelta di $i, j, \ldots, k=1,2$, si sostituiscano in quelle le $M_{i}, N_{j}, \ldots, P_{k}$ alle $M, N, \ldots, P$. Per quanto gia stabilito, sussistono allora le

$$
\left(R M_{i} N_{j} \ldots P_{k}\right)_{V}=\left(R M^{\prime}{ }_{i} N_{j}^{\prime} \ldots P_{k}^{\prime}\right)_{V^{\prime}} ;
$$

e basta combinare linearmente fra loro queste relazioni in modo opportuno, tenendo conto delle (7), per dedurne la (3).

Con argomentazioni simili a quelle dianzi impiegate per la a), si stabili. scono le seguenti proposizioni.

b). - Se valgono le $P \subset M \subset V, Q \subset N \subset V$, allora risulta:

$$
(P Q)_{V}=\left((P N)_{V}(Q M)_{V}\right)_{(M N)} \text {. }
$$

c). - Se valgono le $P \subset V, V^{\prime} \subset V, P^{\prime} \subset P, P^{\prime} \subset P$, allora risulta:

$$
\begin{aligned}
\left(\left(P^{\prime} P^{\prime \prime}\right)_{P} V\right)_{V} & =\left(\left(P^{\prime} V^{\prime}\right)_{V}\left(P^{\prime \prime} V^{\prime}\right)_{V}\right)_{\left(P V^{\prime}\right)_{V}}= \\
& =\left(\left(P^{\prime} V^{\prime}\right)_{V} P^{\prime \prime}\right)_{P}=\left(\left(P^{\prime \prime} V^{\prime}\right)_{V} P^{\prime}\right)_{P} .
\end{aligned}
$$

d). - Se valgono le $P \subset M \subset N \subset V, \boldsymbol{M}^{\prime} \subset M, N^{\prime} \subset N$, allora risulla:

$$
\left(\left(P M^{\prime}\right)_{M} N^{\prime}\right)_{N}=\left(P\left(M^{\prime} N^{\prime}\right)_{N}\right)_{M}
$$

e). - Se valgono le $P \subset M \subset V, P^{\prime} \subset P, P^{\prime \prime} \subset P, M^{\prime} \subset M, M^{\prime \prime} \subset M$, allora risulta:

$$
\left(\left(P^{\prime} M^{\prime}\right)_{M}\left(P^{\prime \prime} M^{\prime \prime}\right)_{M}\right)_{P}=\left(\left(P^{\prime} P^{\prime \prime}\right)_{P}\left(M^{\prime} M^{\prime \prime}\right)_{M}\right)_{M} \text {. }
$$

f). - Se valgono le $P \subset M \subset V, P \subset N \subset V, P^{\prime} \subset P, M^{\prime} \subset M, N^{\prime} \subset N$, allora risulta:

$$
\left(\left(P^{\prime} M^{\prime}\right)_{M} N^{\prime}\right)_{N}=\left(\left(P^{\prime} N^{\prime}\right)_{N} M^{\prime}\right)_{M}
$$


g). - Se valgono le $P=(M N)_{V}, M^{\prime} \subset M, M^{\prime \prime} \subset M, N^{\prime} \subset N, N^{\prime \prime} \subset N$, allora risulta:

$$
\left(\left(M^{\prime} N^{\prime}\right)_{V}\left(M^{\prime \prime} N^{\prime \prime}\right)_{v}\right)_{P}=\left(\left(M^{\prime} M^{\prime \prime}\right)_{M}\left(N^{\prime} N^{\prime \prime}\right)_{N}\right)_{V}
$$

Più precisamente, ciascuna delle (8)-(13) può dapprima venir stabilita nell'ipotesi che le varietà che in essa compaiono siano effettive e soggette ad opportune condizioni di genericità; e si passa poi al caso più generale mediante considerazioni analoghe a quelle svolte dianzi relativamente alla (3), dopo aver constatato che - in virtù delle ipotesi ammesse - i vari membri delle relazioni da stabilire hanno senso a norma delle definigioni dei nn. 3,4 . Lasciando al Lettore i dettagli delle dimostrazioni, ci limiteremo a verificare che in ciascuna delle (8)-(13) i due membri hanno la stessa dimensione virtuale, data ordinatamente da:

$$
\begin{gathered}
p+q-v=(p+n-v)+(q+m-v)-(m+n-v), \\
\left(p^{\prime}+p^{\prime \prime}-p\right)+v^{\prime}-v=\left(p^{\prime}+v^{\prime}-v\right)+\left(p^{\prime \prime}+v^{\prime}-v\right)-\left(p+v^{\prime}-v\right)= \\
=\left(p^{\prime}+v^{\prime}-v\right)+p^{\prime \prime}-p=\left(p^{\prime \prime}+v^{\prime}-v\right)+p^{\prime}-p, \\
\left(p+m^{\prime}-m\right)+n-n=p+\left(m^{\prime}+n^{\prime}-n\right)-m, \\
\left(p^{\prime}+m^{\prime}-m\right)+\left(p^{\prime \prime}+m^{\prime \prime}-m\right)-p=\left(p^{\prime}+p^{\prime \prime}-p\right)+\left(m^{\prime}+m^{\prime \prime}-m\right)-m, \\
\left(p^{\prime}+m^{\prime}-m\right)+n^{\prime}-n=\left(p^{\prime}+n^{\prime}-n\right)+m^{\prime}-m, \\
\left(m^{\prime}+n^{\prime}-v\right)+\left(m^{\prime \prime}+n^{\prime \prime}-v\right)-p=\left(m^{\prime}+m^{\prime \prime}-m\right)+\left(n^{\prime}+n^{\prime \prime}-n\right)-v .
\end{gathered}
$$

Le prime cinque di queste uguaglianze sono identità d'immediato controllo, e l'ultima sussiste in forza della $p=m+n-v$, che subito discende dalla relazione $P=(M N)_{V}$ ammessa in $\mathrm{g}$ ).

Le proposizioni precedenti, e altre consimili, possono venir combinate fra loro per sostituzione, dando luogo a proposizioni più complesse. Al riguardo si potrebbe porre il problema - di cui però qui non ci occupiamo di caratterizzare le varie formule cosi ottenibili, p. es. con l'introduzione di un opportuno simbolismo. Rileviamo ancora che dalle a)-g) si deducono subito altre proposizioni più semplici particolarizzando qualcuna delle varieta che in esse intervengono, come mostrano gli esempi seguenti, a cui potrebbero facilmente venir aggiunti altri.

Dalla a), assumendo $R=V^{\prime}$ e rammentando (n. 3) che $V^{\prime}$ è l'elemento unità dell' anello $\mathfrak{A}_{V^{\prime}}$, si deduce che:

a') Definite le $M^{\prime}, N^{\prime}, \ldots, P^{\prime}$ con le (2), risulta

$$
\left(V^{\prime} M N \ldots P\right)=\left(M^{\prime} N^{\prime} \ldots P^{\prime}\right)_{V^{\prime}} \text {. }
$$

Se nella a) si suppone compaia una sola, $M$, delle $M, N, \ldots, P$, e si assume questa coineidente con $V^{\prime}$, si ottiene che:

$a^{\prime \prime)}$ Supposto $R \subset M \subset V$, risulta :

$$
(R M)_{V}=\left(R\left(M^{[2]}\right)_{V}\right)_{M} .
$$


Basta poi applicare ripetutamente la a) per vedere che

$\left.\mathrm{a}^{\prime \prime \prime}\right)$ Supposto $M^{\prime} \subset M \subset V, N^{\prime} \subset N \subset V, \ldots, P^{\prime} \subset P \subset V$, risulta:

$$
\left(M^{\prime} N^{\prime} \ldots P^{\prime}\right)_{V}=\left(\ldots\left[\left((M N \ldots P)_{V} M^{\prime}\right)_{M} N^{\prime}\right]_{N} \ldots P^{\prime}\right)_{P} \text {. }
$$

Dalla b), fattovi $P=Q, M=N$, segue che :

$\left.b^{\prime}\right)$ Se $P \subset M \subset V$, posto $P^{\prime}=(P M)_{V}, M^{\prime}=\left(M^{[2]}\right)_{V}$ risulla:

$$
\left(P^{[2]}\right)_{V}=\left(P^{[[2]}\right)_{M} \cdot
$$

Facendo in c) $P^{\prime \prime}=P$, si ottiene la seguente proposizione [contenuta anche nella a) come caso particolare]:

$\left.c^{\prime}\right)$ Se valgono le $P^{\prime} \subset P \subset V, V^{\prime} \subset V$, risulta:

$$
\left(P^{\prime} V^{\prime}\right)_{V}=\left(\left(P V^{\prime}\right)_{V} P^{\prime}\right)_{P}
$$

Le ipotesi fatte in d) sono in particolare soddisfatte, ove si assuma:

$$
P \subset V^{\prime} \subset V, P \subset N \subset V, N^{\prime} \subset N, M=M^{\prime}=\left(N V^{\prime}\right)_{V}
$$

Allora è ovviamente $\left(P M^{\prime}\right)_{M}=P$ ed inoltre, in virtù della a), $\left(M^{\prime} N^{\prime}\right)_{N}=\left(N^{\prime} V^{\prime}\right)_{V}$. La (10) così diventa $\left(P N^{\prime}\right)_{N}=\left(P\left(N^{\prime} V^{\prime}\right)_{V}\right)_{M}$, sicchè, scrivendo $M$ ed $M^{\prime}$ in luogo di $N$ ed $N^{\prime}$, vediamo che

$\left.\mathrm{d}^{\prime}\right)$ Se valgono le $P \subset V^{\prime} \subset V, P \subset M \subset V, M^{\prime} \subset M$, risulta

$$
\left(P M^{\prime}\right)_{M}=\left(P\left(M^{\prime} V^{\prime}\right)_{V}\right)_{\left(M V^{\prime}\right)} V^{\prime}
$$

\section{§ II. - Successioni di varietà, loro inverse ed alternanti.}

6. Su di una varietż algebrica $P$, irriducibile, non singolare e di dimensione $p>0$, avremo sovente da considerare delle successioni di elementi (puri, effettivi o virtuali, ed eventualmente nulli)

$$
P_{0}, P_{1}, \ldots, P_{p}
$$

del relativo anello di equivalenza $\mathfrak{A}_{P}$, soddisfacenti alla condizione che le dimensioni di quegli elementi valgano rispettivamente

$$
p, p-1, \ldots, 0 \text {. }
$$

e che il primo $P_{0}$ di essi coincida con l'unità $P$ di $\mathfrak{A}_{P}$. Una successione siffatta verra brevemente denominata una successione di sostegno $P$, e sarà designata con un simbolo del tipo $\{P\}$.

Giova talora di estendere la (1) completandola a destra con un'infinità di zeri, il che conduce ad identificare la più generale successione di sostegno $P$ con una successione infinita

$$
\{P\}=P_{0}, P_{1}, P_{2}, \ldots,
$$

i cui elementi $P_{i}$ siano soggetti alle sole condizioni

$$
P_{0}=P, \quad \operatorname{dim} P_{i}=p-i \quad(i=1,2, \ldots),
$$


ove (in conformità col n. 3) ogni elemento di dimensione negativa si assuma uguale allo zero.

Alla successione $\{P\}$ si può poi utilmente coordinare l'elemento di $\mathfrak{A}_{P}$ somma dei suoi elementi

$$
\mathscr{B}=P_{0}+P_{1}+\ldots+P_{p}
$$

od anche la serie di potenze formali in un'indeterminata $x$ avente la $\{P\}$ quale successione dei coefficienti :

$$
[\{P\}, x]=P_{0}+P_{1} x+P_{2} x^{2}+\ldots .
$$

Si noti che, in forza delle precedenti convenzioni, quest' ultima si riduce di fatto ad un polinomio (di grado $\leq p$ ) nella $x$. Inoltre, in virtù delle (4), (5), manifestamente risulta

$$
\mathfrak{B}=[\{P\}, 1]
$$

sicchè si ha un isomorfismo fra gli elementi $\mathfrak{B}$ di $\mathfrak{A}_{P}$ composti per somma e moltiplicazione e le relative serie di potenze (5).

Le serie di potenze in un'indeterminata $x$ con $i$ coefficienti in un anello commutativo dotato di unita, nell'ipotesi che il loro termine indipendente dalla $x$ sia l'unità, costituiscono un gruppo moltiplicativo abeliano. È subito visto che la stessa proprietà sussiste se l'anello è graduato, e si suppone inoltre che $\mathrm{i}$ suddetti coefficienti siano omogenei ed abbiano gradi in progressione aritmetica di ragione fissa. $\mathrm{Ne}$ discende che:

Gli elementi (4) di $\mathfrak{A}_{P}$ soddisfacenti alle (3), composti moltiplicativamente, costituiscono un gruppo abeliano, isomorfo a quello formato dalle corrispondenti serie di potenze (5).

Dunque, date due successioni $\{P\},\left\{P^{\prime}\right\}$ aventi lo stesso sostegno $P$, se $\mathfrak{B}$ e $\mathfrak{J}^{\prime}$ denotano gli elementi di $\mathfrak{A}_{P}$ che ad esse restano coordinati a norma della (4), il prodotto $\left(\mathfrak{B S}^{\prime}\right)_{P}$ di questi risulta coordinato ad una nuova successione di sostegno $P$, che chiameremo il prodotto di quelle due in $\mathfrak{A}_{P}$ e denoteremo con $\{P\} \cdot\left\{P^{\prime}\right\}$. Ciò equivale ad assumere

$$
\left[\{P\} \cdot\left\{P^{\prime}\right\}, x\right]=[\{P\}, x] \cdot\left[\left\{P^{\prime}\right\}, x\right] .
$$

Si ottiene così che

Le successioni di dato sostegno, composte moltiplicativamente nel modo suddetto, costituiscono un gruppo abeliano (isomorfo a quelli considerati nel precedente enunciato).

Ne consegue che un'arbitraria successione $\{P\}$ di sostegno $P$ ammette in $\mathfrak{A}_{P}$ un' inversa $\{P\}^{-1}$, avente ancora il sostegno $P$, che denoteremo per comodità con

tale che

$$
\{\tilde{P}\}=\tilde{P}_{0}, \tilde{P}_{1}, \tilde{P}_{2}, \ldots,
$$

$$
[\{\tilde{P}\}, x]=[\{P\}, x]^{-1} \text {. }
$$


$\grave{E}$ chiaro che in ogni caso risulta

$$
\{\tilde{\tilde{P}}\}=\{P\} \quad \text { (ossia }\left(\tilde{\tilde{P}}_{i}=P_{i} \text { per } i=0,1,2, \ldots\right) ;
$$

e che dalla

(9)

$$
\{P\} \cdot\left\{P^{\prime}\right\}=\left\{P^{\prime \prime}\right\}
$$

si traggono le

(10) $\quad\{\boldsymbol{P}\}=\left\{\tilde{\boldsymbol{P}}^{\prime}\right\} \cdot\left\{\boldsymbol{P}^{\prime \prime}\right\}, \quad\left\{\boldsymbol{P}^{\prime}\right\}=\{\tilde{\boldsymbol{P}}\} \cdot\left\{\boldsymbol{P}^{\prime \prime}\right\}, \quad\left\{\tilde{\boldsymbol{P}}^{\prime \prime}\right\}=\{\tilde{\boldsymbol{P}}\} \cdot\left\{\tilde{\boldsymbol{P}}^{\prime}\right\}$.

Inoltre, se

$$
\tilde{\mathfrak{E}}=\tilde{P}_{0}+\tilde{P}_{1}+\ldots+\tilde{P}_{p}
$$

è l'elemento di $\mathfrak{A}_{P}$ coordinato alla successione (6), si ha manifestamente:

$$
(\mathfrak{B} \tilde{\mathfrak{S}})_{P}=P \text {. }
$$

Sarà utile talvolta di associare alla successione (2) la

$$
\{\bar{P}\}=P_{0},-P_{1}, P_{2},-P_{3}, \ldots,
$$

che chiameremo l'alternante di quella, assumendo dunque

$$
\bar{P}_{i}=(-1)^{i} P_{i} \quad(i=0,1,2, \ldots) \text {. }
$$

In base alla (5) ed a ciò che precede, ne consegue che è

$$
[\{\bar{P}\}, x]=[\{P\},-x]
$$

e si constatano subito le identità :

$$
\{\overline{\bar{P}}\}=\{P\}, \quad\{\tilde{\bar{P}}\}=\{\overline{\tilde{P}}\}, \quad \overline{\{P\} \cdot\left\{P^{\prime}\right\}}=\{\bar{P}\} \cdot\left\{\bar{P}^{\prime}\right\} \text {. }
$$

7. In virtù del numero precedente, la $\left(9_{6}\right)$ equivale alle relazioni esplicite

$$
P_{i}^{\prime \prime}=\sum_{j=0}^{i} P_{j} P_{i-j}^{\prime}
$$

nelle quali è sottinteso che ciascuno dei prodotti che compaiono a secondo membro dev' essere calcolato su $P$ (operando cioè nell' anello $\mathfrak{z}_{P}$ ). Sulle (1). si constata subito direttamente che, siccome le $P_{i},{P^{\prime}}_{i}$ soddisfano alle $\left(3_{6}\right)$, lo stesso accade per le $P^{\prime \prime}{ }_{1} ;$ e si vede inoltre che $P^{\prime \prime}{ }_{i}$ dipende soltanto dalle $P_{0}, P_{1}, \ldots, P_{i} e$ dalle $P_{0}^{\prime}, P_{1}^{\prime} \ldots, P_{i}^{\prime}$, eppertanto, se $i<p$, non da tutti gli elementi delle successioni $\{P\}$ e $\left\{P^{\prime}\right\}$.

Parimenti, la definizione data nel n. 6 per la successione $\left(6_{6}\right)$ si traduce con le

$$
\sum_{j=0}^{i} P_{j} \tilde{P}_{i-j}=\left\{\begin{array}{lll}
P & \text { se } & i=0 \\
0 & \text { se } & i>0
\end{array} \quad(i=0,1,2, \ldots) .\right.
$$

la prima delle quali esprime semplicemente che $\Theta$

$$
\tilde{P}_{\circ}=P_{0}=P \text {. }
$$


Le $i$ equazioni (2) successive, sono lineari non omogenee nelle $P_{1}, P_{2}, \ldots, P_{i}$, col relativo determinante dei coefficienti uguale all' unità di $\mathfrak{A}_{P}$; da esse si trae subito che è :

$$
\tilde{P}_{i}=(-1)^{i}\left|\begin{array}{cccccc}
P_{1} & P & 0 & 0 & \ldots & 0 \\
P_{2} & P_{1} & P & 0 & \ldots & 0 \\
P_{3} & P_{2} & P_{1} & P & \ldots & 0 \\
\ldots & \ldots & \ldots & \ldots & \ldots & \ldots \\
P_{i} & P_{i-1} & P_{i-2} & P_{i-3} \ldots & P_{1}
\end{array}\right| \quad(i \geq 1)
$$

ossia, ponendo qui successivamente $i=1,2,3,4, \ldots$ e sviluppando,

$$
\begin{gathered}
\tilde{P}_{1}=-P_{1}, \quad \tilde{P}_{2}=\left(P_{1}^{\left[{ }^{[2]}\right)_{P}}-P_{2}, \quad \tilde{P}_{3}=-\left(P_{1}^{\left[{ }^{[}\right]}\right)_{P}+2\left(P_{1} P_{2}\right)_{P}-P_{3},\right. \\
\tilde{P}_{4}=\left(P_{1}^{[1]}\right)-3\left(P_{1}{ }^{[2]} P_{2}\right)_{P}+2\left(P_{1} P_{3}\right)_{P}+\left(P_{z}{ }^{[2]}\right)_{P}-P_{4}, \ldots .
\end{gathered}
$$

Le (3), (4) mostrano che $\tilde{P}_{i}$ dipende soltanto dalle $P_{0}, P_{1}, \ldots, P_{i}$ e che, poichè le $P_{i}$ soddisfano alle $\left(3_{6}\right)$, lo stesso è delle $\tilde{P}_{i}$. Meno immediato sarebbe da quelle dedurre la $\left(8_{6}\right)$ o che ciascuna delle $\left(10_{6}\right)$ equivale alla $\left(9_{6}\right)$. Un'altra riprova dell' utilità delle considerazioni del n. 6 vien fornita dall'esempio che ora daremo, e di cui avremo poi ripetutamente a servirci nel seguito.

Sulla varietà $V$, fissiamo un qualunque numero $s(\geq 1)$ d'ipersuperficie $A$ arbitrarie (effettive o virtuali):

$$
A^{1}, A^{2}, \ldots, A^{s}
$$

e definiamo la successione $\{V\}$ delle

$$
V_{i}=V_{i}(A)=V_{i}^{s}(A)=V_{i}\left(A^{1}, A^{2}, \ldots, A^{s}\right) \quad(i=0,1,2, \ldots)
$$

assumendo

$$
[\{V\}, x]=\prod_{h=1}^{s}\left(1+A^{h} x\right)
$$

dove a secondo membro i prodotti vanno eseguiti su $V$ ed 1 denota l'unità dell' anello $\mathfrak{A}_{V}$, ossia $V$. Dalle $\left(5_{6}\right),(7)$ si trae che è

$$
V_{0}(A)=V \text {, }
$$

che, quando $i$ soddisfa alle $i \geq 1, i \leq v, i \leq s$, la $V_{i}(A)$ uguaglia la somma di tutti $i$ prodotti delle $A$ prese ad $i$ ad $i$ senza ripetizione (ed è quindi una varietà pura di dimensione $v-i$ ), infine che risulta

$$
V_{i}(A)=0 \quad \text { se } i>v \text {, od anche se } i>s .
$$

Ne consegue che le (6) costituiscono una successione $\{V\}$ di sostegno $V$, a norma della definizione del n. 6 . 
Allo scopo di determinare la successione inversa $\{\tilde{V}\}$, osserviamo che dalla (7) si deduce :

$$
\begin{gathered}
{[\{V\},-x]^{-1}=\prod_{h=1}^{s}\left(1-A^{h} x\right)^{-1}=} \\
=\prod_{h=1}^{s}\left(1+A^{h} x+-\left(A^{h}[]^{[2]} x^{2}+\left(A^{h}\right)^{[8]} x^{3}+\ldots\right) .\right.
\end{gathered}
$$

Avato riguardo alle $\left(7_{6}\right),\left(14_{6}\right),\left(15_{6}\right)$, ne consegue che è

$$
\tilde{\bar{V}}_{0}(A)=V
$$

e che, se $i \geq 1, \tilde{\bar{V}}_{i}(A)$ uguaglia la somma di tutti $i$ prodotti delle $A$ prese con ripetizione ad $i$ ad $i$ (talchè risulta $\tilde{V}_{i}(A)=0$ per $i>v$ ). Cio fornisce subito l'espressione esplicita delle $\tilde{V}_{i}(A)$, essendo, in virtù della $\left(13_{6}\right)$,

$$
\tilde{V}_{i}(A)=(-1)^{i} \tilde{\bar{V}}_{i}(A) \text {. }
$$

8. Nella solita varietà ambiente $V$, consideriamo due varietà irriducibili $P$ e $P^{\prime}$, situate in posizione regolare (n. 3) e segantisi lungo una varietà irriducibile $P^{\prime \prime}$ (di dimensione $p^{\prime \prime}=p+p^{\prime}-v \geq 0$ ), talchè risulterà $P^{\prime \prime}=\left(P P^{\prime}\right)_{V}$. Prese due qualunque successioni

$$
P\}=P_{0}, P_{1}, P_{2}, \ldots, \quad\left|P^{\prime}\right|=P_{0}^{\prime}, P_{1}^{\prime}, P_{2}^{\prime}, \ldots
$$

di sostegni $P$ e $P^{\prime}$, possiamo ad esse coordinare una terza successione

$$
\left|P^{\prime \prime}\right|=P_{0}^{\prime \prime}, P_{1}^{\prime \prime}, P_{2}^{\prime \prime}, \ldots,
$$

di sostegno $P^{\prime \prime}$, definendo le $P^{\prime \prime}{ }_{i}$ mediante le $\left(1_{7}\right)$, con la convenzione che ciascuno dei prodotti che ivi compaiono a secondo membro debba ora venir calcolato su $V$. Diremo che la successione (2) è il prodolto delle (1) su $V$, ed esprimeremo tale relazione fra le successioni (1) e (2) mediante la $\left(9_{6}\right)$ od anche, per maggior chiarezza, con la

$$
\left|P^{\prime \prime}\right|=\left(\left\{P|\cdot| P^{\prime} \mid\right)_{V} .\right.
$$

Detto prodotto si definirà anche nel caso escluso in cui sia $p^{\prime \prime}<0$, assumendolo allora uguale allo zero.

La (3) equivale manifestamente alla

$$
\mathfrak{S}^{\prime \prime}=\left(\mathfrak{B} \cdot \mathfrak{B}^{\prime}\right)_{V},
$$

dove $\mathfrak{B}, \mathfrak{B}^{\prime}$ e $\mathfrak{g}^{\prime \prime}$ sono gli elementi di $\mathfrak{A}_{V}$ (dotati anche ordinatamente di rappresentanti in $\mathfrak{A}_{P}, \mathfrak{A}_{P^{\prime}}$ ed $\mathfrak{A}_{P^{\prime \prime}}$ ) coordinati rispettivamente alle successioni (1) e (2) giusta il n. 6. Il prodotto dianzi definito è pertanto commutativo ed associativo, sicchè :

Le successioni aventi per sostegni le varie sottovarietà di una data varietà $V$ costituiscono un semigruppo abeliano moltiplicativo, avente la successione $\nabla, 0,0, \ldots$ come elemento unitò. Quest'ultima verrà pertanto denominata la successione unitaria di $\mathfrak{z}_{V}$. 
Ne consegue che, nel suddetto semigruppo, le successioni che ammettono un' inversa sono quelle di sostegno $V$. L'inversa di una successione $\mid V$ siffatta ha ancora $V$ per sostegno, e non differisce dall'inversa $|\tilde{V}|$ di $|V|$ definita in $\mathfrak{z}_{V}$ giusta $\mathrm{i}$ nn. 6,7 . Si vede inoltre che, nelle condizioni attuali, se (e soltanto se) il sostegno $P^{\prime}$ della seconda successione (1) coincide con la varietà ambiente $V$, la (3) equivale alla

$$
|P|=\left(\left|\tilde{P}^{\prime}\right| \cdot\left|P^{\prime \prime}\right|\right)_{V}
$$

Più in generale, due successioni aventi per sostegni due sottovarietà di $V$, possono venir moltiplicate fra loro su di una sottovarieta di $V$ che contenga quei sostegni; e per prodotti siffatti valgono relazioni analoghe a talune di quelle stabilite nel $n .5$ per prodotti fra sottovarietà di $V$. Cosi, ad esempio, come estensione della d) del n. 5 si ha che:

Prese in $V$ due sottovarietà $M, N$ e tre successioni $\left.\mid P\}, \mid M^{\prime}\right\},\left|N^{\prime}\right| d i$ sostegni $P, M^{\prime}, N^{\prime}$ soddisfacenti alle

$$
P \subset M \subset N \subset V, \quad M^{\prime} \subset M, \quad N^{\prime} \subset N,
$$

sussiste l'uguaglianza:

$$
\left(\left\{|P| \cdot\left\{M^{\prime} \mid\right)_{M} \cdot \mid N^{\prime}\right\}\right)_{N}=\left(|P| \cdot\left(\left\{M^{\prime}\right\} \cdot\left|N^{\prime}\right|\right)_{N}\right\}_{M} \text {. }
$$

Per dimostrarlo, osserviamo che le (4) implicano le

$$
P_{j} \subset M \subset N \subset V, \quad M^{\prime} \subset M, \quad N^{\prime} \subset N
$$

per ogni scelta delle $j, h, k=0,1,2, \ldots$. Pertanto, in forza della $\left(10_{5}\right)$ risulta

$$
\left(\left(P_{j} M_{h}^{\prime}\right)_{M} N_{k}^{\prime}\right)_{N}=\left(P_{j}\left(M_{h}^{\prime}{ }_{h} N_{h}^{\prime}\right)_{N}\right)_{M}
$$

I due membri della (5) sono pertanto due successioni aventi lo stesso sostegno, i loro sostegni essendo precisamente dati dai due membri della relazione fornita dalla (6) per $j=h=k=0$. Per stabilire la (5), basta allora far vedere che, per ogni $i \geq 1$, l' $(i+1)^{\text {mo }}$ elemento di una di quelle successioni coincide con l' $(i+1)^{\text {mo }}$ elemento dell' altra. Ebbene, è subito visto che si ragginge questo scopo sommando a membro a membro le relazioni (6) provenienti dai valori di $j, h, k$ che soddisfano alle

$$
j+h+k=i, \quad j \geq 0, h \geq 0, k \geq 0 .
$$

Con argomentazione simile a quella svolta or oră, dalla e) del n. 5 si deduce che:

Se $P \subset M \subset V$, prese comunque due successioni $\left\{P^{\prime}|,| P^{\prime \prime} \mid\right.$ di sostegno $P$ e due successioni $\left\{M^{\prime}\right\},\left\{M^{\prime \prime}\right\}$ di sostegno $M$, risulta :

(7) $\left.\left(\mid\left\{P^{\prime}|\cdot| M^{\prime} \mid\right)_{M} \cdot\left(\left|P^{\prime \prime}\right| \cdot\left|M^{\prime \prime}\right|\right)_{M}\right)_{P}=\left(|| P^{\prime}|\cdot| P^{\prime \prime} \mid\right)_{P} \cdot\left(\left|M^{\prime}\right| \cdot\left|M^{\prime \prime}\right|\right)_{M}\right)_{M}$.

In particolare, se assumiamo $\left|P^{\prime \prime}\right|=\left|\tilde{P}^{\prime}\right|,\left|M^{\prime \prime}\right|=\left|\tilde{M}^{\prime}\right|$, i prodotti $\left(\left\{P^{\prime}|\cdot| P^{\prime \prime}\right\}\right)_{P}$ e $\left(\left|M^{\prime}\right| \cdot\left\{M^{\prime \prime}\right\}\right)_{M}$ riduconsi alle successioni unitarie di $\mathfrak{Z}_{P}$ ed $\mathfrak{A}_{M}$, onde l'ultima relazione mostra che: 
Se $\left|P^{\prime}\right|,\left|M^{\prime}\right|$ sono due qualunque successioni tali che il sostegno $P$ della prima appartenga al sostegno $M$ della seconda, il prodotto $\left.\left(\left\{P^{\prime}\right\} \cdot \mid M^{\prime}\right\}\right)_{M}$ è una successione di sostegno $P$, la cui inversa in $\mathfrak{A}_{P}$ vale:

$$
\left(\left|\widehat{P^{\prime}|\cdot| M^{\prime}}\right|\right)_{M}=\left(\left|\tilde{P}^{\prime}\right| \cdot\left|\tilde{M}^{\prime}\right|\right)_{\text {a }} \text {. }
$$

Più in generale, basandosi sulla g) del n. 5 , si vede che:

Se $P=(M N)_{V}$, prese comunque due successioni $\left|M^{\prime}\right|,\left|M^{\prime \prime}\right|$ di sostegno $M$ e due successioni $\left|N^{\prime}\right|,\left|N^{\prime \prime}\right|$ di sostegno $N$, risulta:

$$
\left.\left.\left(\left(! M^{\prime}|\cdot| N^{\prime}\right\}\right)_{V} \cdot\left(\mid M^{\prime \prime}\right\} \cdot\left|N^{\prime \prime}\right|\right)_{V}\right)_{P}=\left(\left(\mid M^{\prime}\right\} \cdot\left\{M^{\prime \prime} \mid\right)_{M} \cdot\left(\mid N^{\prime}\right\} \cdot\left\{N^{\prime \prime}||_{N}\right)_{V} \cdot\right.
$$

Pertanto :

Se $|M|,|N|,\{P \mid$ sono tre successioni di sostegni $M, N, P$, aventi rispettivamente $|\tilde{M}|,|\tilde{N}|,|\tilde{P}|$ come inverse in $\mathfrak{A}_{M}, \mathfrak{Z}_{N}, \mathfrak{H}_{P}$,

dalla $\{P\}=(\{M\} \cdot|N|)_{V}$ segue $\left.\left.l a \mid \tilde{P}\right\}=(\{\tilde{M}\} \cdot \mid \tilde{N}\}\right)_{V}$

$e$ questo risultato si estende subito al prodotto di un qualunque numero di successioni.

Notiamo infine che, applicando il n. 5, a), si ottiene che:

Se $\{M\}$ ed $\{N\}$ denotano due successioni qualsiansi coi sostegni $M$ ed $N$ appartenenti a $V$, risulta

$$
\left(|M| \cdot(|N| M)_{V}\right)_{M}=(|M| \cdot|N|)_{V}
$$

\section{§ III. - Un'operazione a tre o più termini fra elementi di $\mathfrak{q}_{V}$ •}

9. Consideriamo in $V$ tre sottovarietà $P, M, N$, la prima delle quali sia non singolare, e supponiamo che le loro dimensioni soddisfino alle

$$
p \geq m+n-v \geq 0 \text {. }
$$

Se le $M, N$ passano semplicemente $\theta$-in modo generico per $P$, la loro interferenza $\langle M N\rangle$ viene ad essere costituita da $P$ e da una residua varietà

di dimensione regolare

$$
Q \doteq\langle M N\rangle-P
$$

$$
q=m+n-v,
$$

lungo la quale le $M, N$ si segano semplicemente. Allora diremo che $Q$ è il prodotto simbolico di $M$ ed $N$ entro $V$, relativo $a \cdot P$, e scriveremo che o

$$
Q=(M N)_{V}^{P}
$$

Più generalmente, se $s(\geq 2)$ varietà $M^{1}, M^{*}, \ldots, M^{s}$ passano in modo generico per $P$, e se è:

$$
p \geq m^{1}+m^{2}+\ldots+m^{s}-(s-1) v \geq 0,
$$

l'intersezione delle $M$ residua a $P$ consta di una varietà $Q$ di dimensione regolare

$$
q=m^{1}+m^{2}+\ldots+m^{s}-(s-1) v
$$


la quale si dirà il prodotto simbolico delle $M$ entro $V$, relativo $a P$, e si designerà col simbolo

$$
Q=\left(M^{1} M^{*} \ldots M^{s}\right)_{V}^{P} .
$$

Il prodotto si definirà anche nel caso escluso in cui sia $q<0$, assumendolo allora uguale allo zero.

$\grave{\mathrm{E}}$ chiaro che un tal prodotto risulta commutativo, ma non necessariamente associativo, rispetto ai fattori $M^{4}, M^{2}, \ldots, M^{s}$. Qualora la dimensione $p$ di $P$ uguagli il numero $q$ definito dalla (6), le $M^{1}, M^{2}, \ldots, M^{s}$ sono in posizione regolare entro $V$ (n. 3), e si segano semplicemente lungo la varietà pura $P+Q$. Tenuto conto della (7) e del n. 3 , si ha quindi che, se $p=q$, risulta :

$$
\left(M^{1} M^{*} \ldots M^{s}\right)_{V}^{P}=\left(M^{1} M^{2} \ldots M^{s}\right)_{V}-P .
$$

10. Ci proponiamo ora di dimostrare il seguente

Teorema. - Se le varietà $P, M^{4}, M^{2}, \ldots, M^{s}$, dianzi considerate, variano su $V$ entro a sistemi di equivalenza, lo stesso accade del prodotto simbolico $\left(7_{\mathrm{g}}\right)$. Questo pù̀ conseguentemente venir altresi considerato come una classe di equivalenza, univocamente determinata dalle classi di equivalenza $P, M^{1}$, $M^{2}, \ldots, M^{s}$, soddisfacenti alle condizioni $P \subset M^{i}$ e $\left(5_{9}\right)$.

Il teorema $\theta$ evidente se $p=q$, essendo allora una conseguenza immediata della $\left(8_{9}\right)$. Basterà dunque dimostrarlo nell' ipotesi che sia

$$
p>q \text {. }
$$

Si ha anzitutto che, se si tien $\mathrm{fisso} P$ e si fanno variare le varietà $M^{4}, M^{2}, \ldots, M^{s}$ per $P$ entro a sistemi elementari (n. 2), anche la varietà $Q$ data dalla $\left(7_{g}\right)$ varia entro ad un sistema elementare. Da qui, tenuto conto del n. 2, si deduce la parte del teorema che concerne la variabilità delle $M^{i}, M^{z}, \ldots, M^{s}$. Allo scopo di estendere il risultato al caso in cui si faccia variare $l$, premettiamo alcune osservazioni che ci verranno utili anche più tardi.

a) Supponiamo che un certo numero $r(\leq s)$ di fattori del prodotto simbolico $\left(7_{9}\right)$ si spezzino nella $P$ ed in una varietà residua, ancora di dimensione $p$; si abbia ad esempio

$$
M^{i}=N^{i}+P \quad\left(m^{i}=n^{i}=p\right) \quad \text { per } i=1,2, \ldots, r .
$$

Allora su $V$ risulta

$$
\left(M^{1} M^{2} \ldots M^{s}\right)_{V}^{P}=\left(N^{1} N^{2} \ldots N^{r}\right)_{V} \cdot\left(M^{r+1} \ldots M^{s}\right)_{V}^{P} .
$$

Questa formula è invero ovvia per $r=1$, in base ai nn. 3, 9; e la si dimostra subito per $r>1$, procedendo per induzione rispetto ad $r$. Rileviamo il 
caso $r=s$, in cui la (2) riducesi alla

$$
\left(M^{1} M^{*} \ldots M^{s}\right)_{V}^{P}=\left(N^{1} N^{2} \ldots N^{s}\right)_{V}=\left(\left(M^{i}-P\right)\left(M^{2}-P\right) \ldots\left(M^{s}-P\right)_{V}\right.
$$

ed è chiaro che, nelle ipotesi attuali, il suddetto teorema si legge senz' altro sulla (3).

b) Date in $V s+1$ varietà $V^{\prime}, M^{4}, \ldots, M^{s}$, supponiamo che la prima sia in posizione regolare rispetto a ciascuna delle altre, e le incontri quindi secondo varietà

$$
M^{\prime} \doteq\left\langle M^{i} V^{\prime}\right\rangle \quad(i=1,2, \ldots, s)
$$

aventi dimensioni regolari

$$
m^{\prime}=m^{i}+v^{\prime}-v
$$

E chiaro che ogni punto comune alle $V^{\prime}, M^{1}, \ldots, M^{s}$ è pure comune alle $M^{\prime \prime}$, $M^{\prime 2}, \ldots, M^{\prime s}$, e viceversa. Inoltre, se le $M$ e la $V^{\prime}$ sono varietà di $V$ passanti in modo generico per $P$, le $M^{\prime}$ risultano varietà di $V^{\prime}$ passanti in modo generico per $P$, le une e le altre avendo una medesima intersezione semplice residua, $Q$, di dimensione

$$
v^{\prime}+\sum_{i=1}^{s} m^{i}-s v=\sum_{i=1}^{s} m^{\prime i}-(s-1) v^{\prime}
$$

e si noti che - supposto valga la (1) - dev'essere $s \geq 2$, poichè altrimenti (ossia se $s=1$ ) la varietà $\left\langle M^{\prime 1} M^{\prime 2} \ldots M^{\prime s}\right)$ sarebbe pura, e non potrebbe quindi coincidere con la $P+Q$. In virtù del n. 9 , su $V$ sussiste pertanto l'equivalenza:

$$
\left(V^{\prime} M^{1} \ldots M^{s}\right)_{V}^{P}=\left(M^{\prime 1} M^{\prime 2} \ldots M^{s}\right)_{V^{\prime}}^{P} \text {. }
$$

Siamo ora in grado di completare la dimostrazione del teorema enunciato in principio, procedendo per induzione rispetto al carattere $\pi=v-p$, il quale deve manifestamente essere positivo. Se $\pi=1$, e cioè se $p=v-1$, la $P$ è un'ipersuperficie di $V$; sicchè ciascuna delle $M$ - dovendo contenere $P$ - si spezza in $P$ ed in un'ipersuperficie residua, ed il teorema è vero in forza di a). Più generalmente, se ciascuna delle $m^{t}$ uguaglia $p$ il teorema sussiste in virtù di $a$ ), sicchè basterà limitarci al naso in cui almeno una delle $m^{i}$ superi $p$.

Se $\pi>1$ ed $m^{4}>p$, possiamo ammettere il teorema per valori inferiori del carattere $\pi$ ed applicare $b$ ). La (6), attualmente, con ovvio cambiamento di notazioni, si scrive nella forma

$$
\left(M^{1} M^{2} \ldots M^{s}\right)_{V}^{P}=\left(M^{\prime 2} \ldots M^{t^{s}}\right)_{M^{1}}^{P} ;
$$

ed il prodotto simbolico che qui figura a secondo membro ha il carattere $\pi^{\prime}=m^{t}-p<\pi$. Ne discende che la varietà $Q$ a primo membro varia in un sistema d'equivalenza, non soltanto se si fanno variare le varietà $M^{1}, M^{2}, \ldots$, $M^{s}$ per $P$ entro a sistemi d'equivalenza su $V$, ma pure se si fa variare la $P$ 
entro a un sistema d'equivalenza su $M^{1}$. Si ha quindi il teorema, dopo aver osservato che ogni varietà equivalente a $P$ può ottenersí applicando alternatamente un conveniente numero di volte le due operazioni di far variare la $P$ entro ad un sistema d'equivalenza su $M^{1}$ e di far variare $M^{1}$, attorno ad una varietà equivalente a $P^{\prime}$ così ottenuta, entro ad un sistema d'equivalenza su $V$.

11. La moltiplicazione simbolica $\left(7_{9}\right)$ di due o più sottovarietà $M^{4}, M^{2}, \ldots, M^{s}$ di $V$, relativa ad una sottovarietà $P$ di $V$, ̀̀ un'operazione che a priori non ha nulla a che vedere con le operazioni dell' anello d'equivalenza $\mathfrak{A}_{V}$. Nel caso banale in cui, con le notazioni del n. 9 , sia $p=q$, v'è tuttavia un semplice legame fra quella moltiplicazione e le operazioni di $\mathfrak{z}_{V}$, espresso dalla $\left(8_{9}\right)$. Estenderemo in seguito siffatto risultato al caso non banale in cui si abbia $p>q$, facendo intervenire certe varietà, opportunamente definite, covarianti delle singole $M^{4}, M^{2}, \ldots, M^{s}, P$ in $V$. Tenuto conto del n. 9 e del teorema del n. 10, l'espressione a cui così perverremo per la differenza

$$
\left(M^{1} M^{2} \ldots M^{s}\right)_{V}-\left(M^{1} M^{2} \ldots M^{s}\right)_{V}^{P},
$$

la quale è una varietà (virtuale) pura, di dimensione $q$, definita a meno di un'equivalenza, potrà dirsi l'equivalenza funzionale di $P$ nell'intersezione delle varietà $M^{\prime}, M^{*}, \ldots, M^{s}$ passanti semplicemente per quella.

Si ottiene una varietà $Q^{\prime}$ equiralente alla (1), quale intersezione residua a $Q$ di varietà $M^{\prime 1}, M^{\prime 2}, \ldots, M^{\prime s}$ rispettivamente equivalenti alle $M^{1}, M^{2}, \ldots$, $M^{s}$ e passanti genericamente per $Q$. E chiaro che, se le $M^{\prime 1}, M^{\prime \prime}, \ldots, M^{\prime s}$ variano tendendo alle $M^{1}, M^{2}, \ldots, M^{s}$, la suddetta varietà $Q^{\prime}$ varia mantenendosi equivalente alla (1) e tendendo ad una sottovarietà di l'. Pertanto:

La varietà virtuale (1) appartiene ad $\mathfrak{A}_{P}$, ossia può dirsi virtualmente contenuta in $P$.

Nonostante ciò che si è detto al principio del presente numero, possiamo osservare che v'è una certa analogia fra i prodotti in $\mathfrak{A}_{V}$ e le moltiplicazioni simboliche di cui al n. 9. Questa analogia, ginstificata dal teorema che daremo in nota nel numero successiro, rende conto del perchè da taluno dei risultati del n. 5 , ove compaiono soltanto operazioni in $\mathfrak{A}_{V}$, possano trarsi uno o più risultati consimili inerenti alle moltiplicazioni simboliche. Cosł̀, ad esempio, la $\left(6_{10}\right)$ - stabilita in $\left.b\right)$ al n. 10 - può venire considerata come un' estensione della proposizione data in $\left.a^{\prime}\right)$ nel $n$. 5. Un altro modo di estendere tale proposizione è quello espresso dalla

$$
\left(V^{\prime} \cdot\left(M^{1} M^{2} \ldots M^{s}\right)_{V}^{P}\right)_{V}=\left(M^{\prime 1} M^{\prime 2} \ldots M^{\prime s}\right)_{V^{\prime}}^{P^{\prime}},
$$

dove le notazioni sono le stesse del n. $10, b)$ e si è posto $P^{\prime}=\left(I^{\prime} V^{\prime}\right)_{V}$. 
Per dimostrare la (2), è lecito supporre le $M^{1}, M^{2}, \ldots, M^{s}$ passanti in modo generico per $P$, e le $P, V^{\prime}$ situate in posizione regolare entro $V$; si possono inoltre assumere le $M^{\prime i}$ definite dalle $\left(4_{10}\right)$, e $P^{\prime}$ coincidente con $\left\langle P V^{\prime}\right\rangle$. Allora è chiaro che l'intersezione delle $M^{1}, M^{2}, \ldots, M^{s}$, residua a $P$, vien segata da $V^{\prime}$ secondo l'intersezione delle $M^{\prime \prime}, M^{\prime 2}, \ldots, M^{\prime s}$, residua a $P^{\prime}$. Poichè, in forza delle $\left(\overline{0}_{10}\right)$, i due membri della (2) hanno la stessa dimensione virtuale

$$
v^{\prime}+\left(m^{1}+m^{2}+\ldots+m^{s}-(s-1) v\right)-v=m^{\prime 1}+m^{\prime 2}+\ldots+m^{\prime s}-(s-1) v^{\prime},
$$

cosi, tenuto conto dei nn. 3,9, ne consegue la (2). Si sarebbe potuto raggiunger lo scopo più rapidamente, applicando ill teorema dato nella nota ("1) al seguente n. 12 .

12. Conservando le notazioni del primo capoverso del n. 9, dimostriamo che

Le due varietà $P$ e $Q$, ivi considerate, sono appoggiate l'una all'altra lungo una varietà pura (eventualmente nulla):

(1)

di dimensione $r=q-1$.

$$
R=\langle P Q\rangle
$$

A tal fine, trasformiamo birazionalmente $V$ in una varieta $V^{\prime}$ (di dimensione $v$ ), mediante una dilatazione di base $P\left({ }^{10}\right)$. Con ciò $P$ mutasi in un'ipersuperficie $P^{\prime}$ non singolare di $V^{\prime}$; ed alle $M, N$ corrispondono su $V^{\prime}$ due sottovarietà $M^{\prime}, N^{\prime}$, ancora di dimensioni $m, n$, che non giacciono nell'ipersuperficie $P^{\prime}$ e quindi incontrano questa lungo varietà $M^{\prime}{ }_{1}, N^{\prime}$, aventi rispettivamente le dimensioni $m-1, n-1$. Osserviamo ora che, a norma della $\left(2_{g}\right)$, alla varietà $Q$ di $V$ viene a corrispondere si $V^{\prime}$ una varieta pura $Q^{\prime}$, di dimensione $q$, intersezione semplice delle $M^{\prime}, N^{\prime}\left({ }^{11}\right)$. La $Q^{\prime}$ non appartiene all'ipersuperficie $P^{\prime}$ e quindi incontra quest' ultima lungo una varietà pura $R$, di dimensione $q-1$, soddisfacente manifestamente alla:

$$
R^{\prime} \doteq\left(P^{\prime} Q^{\prime}\right) \doteq\left\langle M_{1}^{\prime} N_{1}^{\prime}\right\rangle
$$

In viriù della (1), questa $R^{\prime}$ viene mutata nella $R$ dalla corrispondenza fra $V^{\prime}$ e $V$ (la quale opera univocamente senza eccezioni nel passaggio da $V^{\prime}$ a $V$ ); pertanto il risultato da stabilire ne seguirà, dopo aver dimostrato ohe il riferimento subordinato fra $R$ ed $R^{\prime}$ da quella corrispondenza è birazionale.

(10) Relativamente a siffatte trasformazioni, ved. B. SEGRE [6], § 1.

(11) Poichè $Q^{\prime}=\left(M^{\prime} N^{\prime}\right)_{V^{\prime}}$, cosı̀ (n. 3) $Q^{\prime}$ varia su $V^{\prime}$ entro ad un sistema d'equivalenza se lo stesso è di $M^{\prime}$ ed $N^{\prime}$. Ritornando a $V$, da qui si deduce una nuova dimostrazione del fatto (n. 10) che, al variare di $M$ ed $N$ per $P$ entro a sistemi d'equivalenza su $V$, anche $Q$ varia in un sistema d'equivalenza. Si noti ehe l'argomentazione del testo prova, più generalmente, che:

Una dilatazione di $V$ in $\nabla^{\prime}$, di base $P$, muta ogni varietà $\left(M^{1} M^{2} \ldots M^{s}\right)_{V}^{P}$ in una varietà del tipo $\left(M^{\prime} M^{\prime 2} \ldots M^{\prime}\right)_{V \prime}$, dove le $M^{\prime}$ hanno un opportuno comportamento rispetto alla varietà $P^{\prime}$ in cui $P$ si dilata passando da $V$ a $V^{\prime}$ (su ciò, cfr. quanto specificato nel testo al capoverso successivo, ed anche il n. 16'). 
Ricordiamo, a questo scopo, che si può prendere un modello proiettivo di $V^{\prime}$, su cui l'ipersuperficie $P^{\prime}$ risulti luogo di $\infty^{n}$ spazi lineari $O^{\prime}$, di dimensione $v-p-1$, ciascuno trasformato di un punto $O$ di $P$. Il generico di tali spazi $O^{\prime}$ sega le $M^{\prime}, N^{\prime}$ secondo spazi lineari $O_{M^{\prime}}^{\prime}, O_{N^{\prime}}^{\prime}$, i quali corrispondono agli intorni del punto $O$ sulle $M, N$, ed hanno rispettivamente le dimensioni $m-p-1, n-p-1$. Ora è chiaro che i luoghi degli $\infty^{p}$ spazi $O_{M}^{\prime}, O_{N^{\prime}}^{\prime}$ sono le suddette varietà $M_{i}^{\prime}, N_{1}^{\prime}$. Inoltre, mentre per un generioo $O$ questi due spazi subordinati di $O^{\prime}$ sono sghembi fra loro, d'accordo col fatto che in virtù della $\left(1_{9}\right)$ risulta

$$
(m+p-1)+(n-p-1)-(v-p-1)<0,
$$

avuto riguardo alla genericità della varietà $M$ ed $N$ per $P$ si ha che due di detti spazi che s'incontrino hanno generalmente un sol punto a comune. D'altro canto, tenuto conto della (2), si vede che $O_{M^{\prime}}^{\prime}$ ed $O_{N^{\prime}}^{\prime}$ hanno punti a comune (necessariamente situati su $R^{\prime}$ ) se, e soltanto se, $O$ giace su $R$, nel qual caso tali punti sono precisamente i trasformati di $O$ nel riferimento fra $R$ ed $R^{\prime}$. Ne consegue che ad un generico punto $O$ di $R$ corrisponde un sol punto su $R^{\prime}$, e ciò prova l'asserto.

Mostreremo che :

Se le varietà $M, N, P$ descrivono su $V$ dei sistemi d'equivalenza, in guisa che $M$ ed $N$ continuino a passare per. $P$, anche la varietò $R-d i$ cui al precedente enunciato - varia su $V$ entro ad un sistema d'equivalenza.

Scegliamo comunque in $V$ una generica ipersuperficie $V^{\prime}$ passante semplicemente per $P$, e quindi pure per $R$. La $V^{\prime}$ non conterrà alcuna componente della varietà $Q$ definita dalla $\left(2_{g}\right)$, e segherà $Q$ lungo una varietà pura, di dimensione regolare $q-1=r$, composta della $R$ e di un' ulteriore varietà $R_{1}$. E chiaro che $R_{1}$ risulta l'intersezione delle $M, N, V^{\prime}$ residua a $P$, in guisa che si può scrivere:

$$
R_{\mathrm{t}}=\left(M N V^{\prime}\right)_{V}^{P}, \quad\left(Q V^{\prime}\right)_{V}=R+R_{4}
$$

Ne consegue la

$$
R=\left(Q V^{\prime}\right)_{V}-\left(M N V^{\prime}\right)_{V}^{P}
$$

per ogni scelta di $V^{\prime}$ soggetta alle condizioni indicate. E dalla (3) si trae subito quanto asserito, poggiando sui nn. 3,10 .

I precedenti risultati, concernenti la varietà (1) relativa ad un prodotto simbolico $\left(4_{9}\right)$ di due fattori, si trasportano senza difficoltà all'analoga varietà, definibile ancora con la (1), relativa ad un prodotto simbolico $\left(7_{g}\right)$ di un qualunque numero di fattori. In seguito potremo dunque valerci di tale estensione. 
CAPITOLO SECONDO

VARIETA COVARIANTI D'IMMERSIONE DI UNA VARIETA IN UN'ALTRA

\section{§ I. - La successione covariante di dato sostegno.}

13. Fissata una qualunque sottovarieta $P$ irriducibile e non singolare di $V$ $(0<p<v)$, scegliamo un intero (positivo) $s$, variabile nell'intervallo

$$
v-p \leq s \leq v
$$

in corrispondenza al quale assumiamo

$$
t=s+p-v \quad(0 \leq t \leq p) .
$$

Prese inoltre in $V$ comunque $s$ ipersuperficie passanti genericamente per $P$ :

$$
A^{1}, A^{2}, \ldots, A^{s}
$$

poniamo $V_{0}^{s}=V$ e denotiamo con $V_{i}^{s}=V_{i}^{s}(A)$ la somma dei prodotti delle (3) combinate ad $i$ ad $i$ senza ripetizione $(i=1,2, \ldots, s)$. Possiamo allora defi. nire le varietà $P_{V, i}$ col porre:

$$
\left(A^{1} A^{3} \ldots A^{s}\right)_{V}-\left(A^{1} A^{2} \ldots A^{s}\right)_{V}^{P}=\sum_{i=0}^{t} P_{V, i} V_{t-i}^{s}(A)
$$

Pì̀ precisamente, tenuto conto della $\left(8_{9}\right)$, l'equazione fornita dalla (4) per $s=v-p$ porge

$$
P_{V, 0}=P \text {. }
$$

Inoltre le equazioni date dalle (4) per $s=v-p+1, v-p+2, \ldots, v$ determinano successivamente - e in modo unico - le varietà $P_{V, 1}, P_{V, 2}, \ldots, P_{V, p}$ quali elementi di $\mathfrak{A}_{V}$; ed anzi, poichè (n: 11) il primo membro della (4) 仓̀ una varietà pura di dimensione $q=v-s=p-t$ contenuta in $P$, cosi $P_{V, i}$ risulta nel modo anzidetto una varietà pura di dimensione $p-i$, appartenente a $P$. Con la terminologia del n. 6, possiamo dunque dire che le (4) definiscono una successione

$$
\left\{P_{V}\right\}=P_{V, 0}, P_{V, 1}, P_{V, 2}, \ldots
$$

di sostegno $P$.

Così, ad esempio, nel caso particolare in oui si assumano le (3) equivalenti ad una stessa ipersuperficie $A$ di $V$, posto per abbreviare

$$
D_{s+p-v}=\left(A^{1} A^{2} \ldots A^{s}\right)_{V}-\left(A^{1} A^{2} \ldots A^{s}\right)_{V}^{P}-\left(\begin{array}{c}
s \\
s+p-v
\end{array}\right)\left(P A^{[s+p-v]}\right)_{V}
$$


(talchè $D_{s+p} v$ è una varietà pura di dimensione $v-s$ appartenente a $P$ ), risulta :

$$
P_{V, i}=\mid \begin{array}{llll}
V & 0 & 0 & \ldots D_{1} \\
\left(\begin{array}{c}
v-p+2 \\
1
\end{array}\right) A & V & 0 & \ldots D_{2} \\
\left(\begin{array}{c}
v-p+3 \\
2
\end{array}\right) A^{[2]} & \left(\begin{array}{c}
v-p+3 \\
1
\end{array}\right) A & V & \ldots D_{3} \\
\ldots \ldots \ldots \ldots & \ldots \ldots \ldots \ldots
\end{array} .
$$

A priori la successione (6), oltre che da $P$ e $V$, dipende dalla scelta delle ipersuperficie (3). È assai notevole che, come dimostreremo nel numero successivo, essa risulta invece indipendente da tale scelta. La (6) costituisce pertanto una successione covariante d'immersione di $P$ in $V$, la corarianza sussistendo sia di fronte alle trasformazioni birazionali senza eccezioni della $V$, che alla variazione di $P$ su $V$ entro ad un sistema d'equivalenza. Quando non vi sia luogo ad equivoco, chiameremo tale successione brevemente la successione covariante relativa a $P$ (o di sostegno $P$ ) e adotteremo per essa la notazione

$$
\{P\}=P_{0}, P_{1}, P_{2}, \ldots
$$

già usata in senso più lato nel n. 6, per designare una successione qualsiasi di sostegno $P$.

Altre possibili definizioni per la suddetta successione covariante $\left\{P_{V}\right\}$ risulteranno dai nn. $16^{\prime}$ e 24.

14 Dimostreremo dapprima il fatto asserito nel penultimo capoverso, per il caso in cui $P$ sia un'ipersuperficie di $V(p=v-1)$. Allora occorre definire le ipersuperficie $\left(3_{13}\right)$ con equazioni del tipo:

$$
A^{i} \doteq P+B^{i} \quad(i=1,2, \ldots, s)
$$

dove anche le $B$ sono ipersuperficie; sicchè, avuto riguardo al n. 10, $a$ ) ed al n. 13 , otteniamo $t=s-1$ ed inoltre

$$
\begin{aligned}
\left(A^{1} A^{2} \ldots A^{s}\right)_{V}^{P} & =\left(B^{1} B^{z} \ldots B^{s}\right)_{V}=\left(\left(A^{1}-P\right)\left(A^{z}-P\right) \ldots\left(A^{s}-P\right)\right)_{V}= \\
& =\left(A^{1} A^{2} \ldots A^{s}\right)_{V}-\sum_{i=0}^{t}(-1)^{i}\left(P^{[i+1]}\right)_{V} V_{t-i}^{s}(A) .
\end{aligned}
$$

Poichè, come s'è visto, la successione $\left(6_{13}\right)$ resta definita univocamente dalle $\left(4_{13}\right)$, il confronto delle $\left(4_{13}\right)$ con le precedenti equazioni porge le uguaglianze (1) $\quad P_{V, i}=(-1)^{i}\left(P^{[i+1]}\right)_{V} \quad(p=v-1) ;$ e si noti che queste sussistono per tutti i valori $i=0,1,2, \ldots$ (anche per $i \geq v$ ), in virtù delle convenzioni fatte nei nn. 3,6 . 
L'intento prefissoci è cosi raggiunto per $p=v-1$, in quanto è ovvio che i secondi membri delle (1) non dipendono dalle $A$. Le (1) - tenuto anche conto del n. 7 - mostrano più precisamente che, chiamando (secondo l' uso) i-ma varietà caratteristica di una qualunque varietà $P$ di $V$ la potenza $(i+1)$-ma di $P$ come elemento di $\mathfrak{A}_{V}$,

La successione covariante relativa ad un'ipersuperficie $P$ di $V$ non $\dot{e}$ che l'alternante della successione delle varietà caratteristiche di $P$ in $V$, ed ha quindi come inversa (n. 7) la successione $P, P^{[2]}, 0,0, \ldots$.

15. Possiamo ora stabilire in tutta generalita che, come asserito alla fine del n. 13,

La varietà $P_{V, t}$, definita nel $n .13$ per $t=0,1,2, \ldots$, non dipende dalla scelta delle ipersuperficie $\left(3_{13}\right)$, e quindi dipende soltanto da $P, V$ e dall'indice $t$.

Questo risultato vale sempre senz'altro per $t=0$, in forza della $\left(5_{13}\right)$. Esso inoltre, in virtù del n. 14, sussiste per ogni valore di $t$ nell' ipotesi che il carattere

$$
\pi=v-p
$$

della coppia $P, V$ raggiunga il valore minimo $\pi=1$. Potremo quindi assumere per i caratteri $\pi, t$ valori soddisfacenti alle $\pi \geq 2, t \geq 1$, onde la $\left(2_{13}\right)$ fornisce $s \geq 3$; inoltre, procedendo per doppia induzione completa rispetto a $t, \pi$, ammetteremo la validità del teorema enunciato per i valori inferiori dell'indice $t$ e per quel valore di $\pi$, come pure per $\mathrm{i}$ valori inferiori di $\pi$ e $t$ arbitrario. Allora la $\left(4_{42}\right)$ definisce $P_{V, t}$ come funzione simmetrica delle $A$ : sicchè l'asserto seguirà subito ove si mostri che $P_{V, t}$ non dipende da una almeno delle $A$.

Scegliamo una delle $\left(3_{13}\right)$, p. es. l'ultima, e, per semplicità di scrittura, poniamo

$$
V^{\prime}=A^{s}, \quad\left(A^{\prime} V^{\prime}\right)_{V}=A^{\prime i} \quad(i=1,2, \ldots, s-1)
$$

Allora manifestamente risulta:

$$
V_{i}^{s}(A)=\nabla_{i}^{s-1}(A)+V^{\prime} V_{i-1}^{s-1}(A) \quad(i=0,1,2, \ldots)
$$

ore si convenga di assumere

$$
\nabla_{-1}(A)=0 \text {. }
$$

Inoltre, dal n. 5, a), a') e dal n. 10, b) rispettivamente discendono le:

$$
\begin{aligned}
\left(P_{V, i} \cdot V_{j}^{s-1}(A)\right)_{V} & =\left(P_{V, i} \cdot V_{j}^{\prime s-1}\left(A^{\prime}\right)\right)_{V^{\prime}} \\
\left(A^{1} A^{2} \ldots A^{s}\right)_{V} & =\left(A^{\prime 1} A^{\prime 2} \ldots A^{\prime s-1}\right)_{V^{\prime}} \\
\left(A^{1} A^{2} \ldots A^{s}\right)_{V}^{P} & =\left(A^{\prime 1} A^{\prime 2} \ldots A^{s-1}\right)_{V^{\prime}}^{P}
\end{aligned}
$$

Dunque la $\left(4_{43}\right)$ riducesi alla seguente equivalenza su $V^{\prime}$ :

$\left(A^{\prime \prime} A^{\prime 2} \ldots A^{\prime s-1}\right)_{V^{\prime}}-\left(A^{\prime 1} A^{\prime 2} \ldots A^{\prime s-1}\right)_{V^{\prime}}^{P}=\sum_{i=0}^{t} P_{V ; i} V_{t-i}^{\prime s-1}\left(A^{\prime}\right)+\sum_{i=0}^{t}\left(V^{\prime} P_{V, i}\right)_{V} V_{t-i-1}^{\prime s-1}\left(A^{\prime}\right)$. 
Avuto riguardo alla (3), nell' ultima sommatoria basta far variare l'indice $i$ da 0 a $t-1$; pertanto, scrivendo in essa $i-1$ in luogo di $i$ ed assumendo per definizione

$$
P_{V,-1}=0,
$$

l'altima relazione diventa:

(5) $\left(A^{\prime 1} A^{\prime 2} \ldots A^{\prime s-1}\right)_{V^{\prime}}-\left(A^{\prime 1} A^{\prime 2} \ldots A^{\prime s-1}\right)_{V^{\prime}}^{P}=\sum_{i=0}^{t}\left[P_{V, i}+\left(V^{\prime} P_{V, i-1}\right)_{V}\right] V_{t-i}^{\prime s-1}\left(A^{\prime}\right)$.

D'altro canto, il carattere (1) relativo alla coppia $P, V^{\prime}$ vale

$$
\pi^{\prime}=v^{\prime}-p=(v-1)-p<\pi ;
$$

sicchè, in relazione ad essa ed alle $s=s-1$ ipersuperficie

$$
A^{\prime 1}, A^{\prime 2}, \ldots, A^{\prime s-1}
$$

di $V^{\prime}$, le quali - a norma delle (2) - contengono $P$ genericamente, per l'ammessa induzione avremo

$$
\left.\left(A^{\prime 1} A^{\prime 2} \ldots A^{\prime s-1}\right)_{V^{\prime}}-\left(A^{\prime \prime} A^{\prime 2} \ldots A^{s-1}\right)_{V^{\prime}}^{P}=\sum_{i=0}^{t^{\prime}} P_{V^{\prime}, i} V_{t^{\prime}-i}^{s-1}\left(A^{\prime}\right) \quad \text { (su } V^{\prime}\right),
$$

dove le $P_{V^{\prime}, i}$ dipendono soltanto da $P, V^{\prime}$ ed è inoltre

$$
t^{\prime}=s^{\prime}+p-v^{\prime}=(s-1)+p-(v-1)=t .
$$

Basta quindi confrontare le (5), (6) per vedere che risulta :

$$
\sum_{i=0}^{t}\left[P_{V, i}+\left(V^{\prime} P_{V, i-1}\right)_{V}-P_{V^{\prime}, i}\right] V_{t-i}^{\prime s-1}\left(A^{\prime}\right)=0
$$

(su $\left.V^{\prime}\right)$.

Osserviamo ora che l'espressione che compare nella (7) entro parentesi quadre è identicamente nulla per $i=0$, in forza delle $\left(5_{13}\right)$, (4). Proveremo per induzione ch' essa si annulla per tutti i valori di $i$, e cioè che - su $V^{\prime}$, e perciò anche su $V$ - si ha

$$
P_{V, i}=P_{V^{\prime}, i}-\left(V^{\prime} P_{V, i-1}\right)_{V} \quad(i=0,1,2, \ldots) .
$$

All' uopo basta mostrare che la (8) sussiste per $i=t$, nell'ipotesi ch'essa valga per $i=0,1, \ldots, t-1$; ora questo segue subito dalla (7), dopo aver rammentato che $V_{0}^{\prime s-1}\left(A^{\prime}\right)=V^{\prime}$ è l'elemento unità dell'anello d'equivalenza di $V^{\prime}$. Si ha pertanto:

$$
P_{V, t}=P_{V^{\prime}, t}-\left(V^{\prime} P_{V, t-1}\right)_{V}
$$

sicchè, nelle ipotesi attuali. $P_{V, t}$ viene a dipendere al più da $P, V \ominus V^{\prime}$, ed è quindi indipendente da $A^{\mathrm{l}}$; per quanto già osservato, eiò è sufficiente per stabilire l' asserto. 
Si noti da ultimo che le equazioni (8) determinano univocamente le $P_{V}$ in funzione delle $P_{V^{i}}$, equivalendo esse precisamente alle

$$
P_{V, i}=\sum_{j=0}^{i}(-1)^{i-j}\left(P_{V^{\prime}, j} V^{\prime}[i-j]\right)_{V} \quad(i=0,1,2, \ldots) .
$$

Di più, a norma di ciò che precede, i secondi membri di queste equazioni risultano indipendenti da $V^{\prime}$ (che è una qualunque ipersuperficie di $V$ passante per $P$ ).

\section{§ II. - Prime applicazioni ed esempi.}

16. Una prima applicazione dei precedenti concetti e risultati, la quale sarà poi largamente generalizzata, vien fornita dal teorema:

Se $s(\geq v-p)$ ipersuperficie $A^{1}, A^{*}, \ldots, A^{s} d i V$ passano genericamente per $P$ e si segano ulteriormente secondo una varietà $Q$, di dimensione $q=v-s>0$, allora $Q$ si appoggia a $P$ lungo una varietò $R$ ldi dimensione $r=q-1)$ data dall' equivalenza

$$
R=\sum_{i=0}^{t+1} P_{V, i} V_{t-i+1}^{s}(A)
$$

ove $t=s+p-v$.

In virtù del n. 12, presa una generica ipersuperficie $V^{\prime}$ di $V$ passante per $P$, risulta :

$$
R=\left(Q V^{\prime}\right)_{V}-\left(A^{1} A^{2} \ldots A^{s} V^{\prime}\right)_{V}^{P}
$$

Inoltre, in base alla definizione del simbolo $\left(7_{9}\right)$, la $\left(4_{13}\right)$ porge

$$
Q=\left(A^{1} A^{2} \ldots A^{s}\right)_{V}-\sum_{i=0}^{t} P_{V, i} V_{t-i}^{s}(A) \text {. }
$$

Infine, applicando ancora la $\left(4_{13}\right)$, si ottiene:

$$
\left(A^{1} A^{z} \ldots A^{s} V^{\prime}\right)_{V}^{P}=\left(A^{1} A^{2} \ldots A^{s} V^{\prime}\right)_{V}-\sum_{i=0}^{t+1} P_{V, i}\left[V_{t-i+1}^{s}(A)+V^{\prime} V_{i-i}^{s}(A)\right] .
$$

Basta allora eliminare $Q$ ed $\left(A^{1} A^{2} \ldots A^{s} V^{\prime}\right)_{V}^{P}$ fra queste tre equazioni, ricordando la $\left(3_{15}\right)$, per dedurne la (1).

16'. Ci proponiamo ora di dare una nuova definizione, di carattere topologico, della successione covariante d'immersione $\left|P_{V}\right|$ di $P$ in $V$. Se $p=v-1$, la definizione fornita dalla $\left(1_{14}\right)$ ha gia il requisito voluto: mostreremo come, nell'ipotesi in cui sia $p<v-1$, ci si possa sostanzialmente ricondurre al caso $p=v-1$ (trattato appunto nel n. 14), sostituendo a $V$ la varietà $V^{\prime}$ che si deduce dalla $V$ applicandole una dilatazione $D$ di base $P$. 亡̀ chiaro anzitutto che quest'ultimo concetto è di natura topologica: 
invero [ofr. loc. cit. in $\left({ }^{10}\right)$ ], la suddetta varietà $V^{\prime}$ (di dimensione $v$ ) si può pensare ricavata da $V$ col tagliarla lungo $P$ e col chiudere la varietà aperta $V-P$. così ottenuta, mediante la varietà fibrata $P^{\prime}$ (di dimensione $v-1$ ) avente per base $P$ e per fibre degli spazi proiettivi complessi $O^{\prime}$, di dimensione

$$
0=v-p-1 \text {. }
$$

Incominciamo con l'osservare che un'ipersuperficie $A$ di $V$, che non passi per $P$, viene trasformata da $D$ in un'ipersuperficie $A^{\prime}$ segante $P^{\prime}$ secondo una varietà fibrata, composta con le fibre $O^{\prime}$ anzidette; si ha pertanto:

$$
\left(A^{\prime} O^{\prime}\right)_{V^{\prime}}=0 \text {. }
$$

Se $A$, variando su $V$ entro ad un sistema d'equivalenza, tende ad una posizione limite passante genericamente per $P$, la $A^{\prime}$ varia pure su $V^{\prime}$ entro ad un sistema d'equivalenza e, al limite, si spezza in $P^{\prime}$ ed in un'ipersuperficie $B^{\prime}$ residua, la quale sega le fibre $O^{\prime}$ di $P^{\prime}$ secondo varietà $\bar{O}$ isomorfe a loro iperpiani (n. 12). Si ha così :

$$
A^{\prime}=P^{\prime}+B^{\prime}, \quad\left(B^{\prime} O^{\prime}\right)_{V^{\prime}}=\bar{O}
$$

ed inoltre, tenuto conto della (1), la varietà

$$
(\bar{O}[0])_{O r}
$$

si riduce ad an sol punto di $O^{\prime}$ (contato semplicemente).

Dalle (2), (3) si trae l' equivalenza

$$
\left(O^{\prime} P^{\prime}\right)_{V}=-\bar{O}
$$

e poichè il secondo membro della (5) è una varietà (virtuale) non effet. tiva, ne consegue che

La varietà caratteristica $\left(P^{[2]}\right)_{V^{\prime}}$ di $P^{\prime}$ in $V^{\prime}$ ed anche, più generalmente, ciascuna delle $\left(P^{\prime[\hat{i}+1]}\right)$ ammettono come rappresentanti delle sottovarietà (virtuali) di $P^{\prime}$ composte di $\left(\infty^{p-i}\right)$ fibre $O^{\prime}$. Si ha dunque $\left(P^{\prime[i+1]}\right)_{V^{\prime}}=0$ per tutti $i$ valori $d i \quad i>p$.

Se $M$ è una qualunque varietà di $P^{\prime}$, denoteremo con $[M]^{*}$ il luogo delle fibre $O^{\prime}$ di $P^{\prime}$ passanti per i vari punti di $M$, ossia lá varietà che si ricava da $M$ quando le si applichino successivamente le trasformazioni $D^{-1}$ e $D$. Ricordando ciò ohe dianzi si è detto relativamente alla (4), ed usufruendo della (5), si vede che - per $k>0$ - risulta

$$
\left[\left(P^{\prime[k]}\right)_{V^{\prime}}\right]^{*}=(-1)^{o}\left(P^{\prime[k-0]}\right)_{V^{\prime}} \text {. }
$$

Ciò premesso, rifacciamoci alle considerazioni del n. 16 , e siano

$$
A^{i^{\prime}}=P^{\prime}+B^{i^{\prime}} \quad(i=1,2, \ldots, s)
$$


le ipersuperficie di $V^{\prime}$ trasformate delle $A^{i}$, ed $R^{\prime}$ la varietà di $P^{\prime}$ (fibrata con le $O^{\prime}$ ) trasformata di $R$ mediante $D$. Allora, con ovvi significati per i simboli, dalla $\left(1_{16}\right)$ si trae l'equivalenza

$$
R^{\prime}=\sum_{i=0}^{t+1}\left(P_{V, i}\right)^{\prime} V_{t-i+1}^{\prime s}\left(A^{\prime}\right) .
$$

D'altro canto, le $\left(2_{13}\right)$, (1) porgono

$$
s-o=t+1
$$

sicchè in virtù del n. 12 , e tenuto conto delle (7), (6), si rêle che ̀े :

$$
\begin{aligned}
& R^{\prime}=\left[\left(P^{\prime} B^{\prime \prime} B^{2^{\prime}} \ldots B^{s^{\prime}}\right)_{V^{\prime}}\right]^{*}=\left[P^{\prime}\left(A^{1 \prime}-P^{\prime}\right)\left(A^{2^{\prime}}-P^{\prime}\right) \ldots\left(A^{s^{\prime}}-P^{\prime}\right)\right]^{*}= \\
& =\sum_{i=0}^{t+1}(-1)^{s-t+i-1}\left[\left(P^{\prime[s-t+i]}\right)_{V}\right]^{*} V_{t-i+1}^{\prime s}\left(A^{\prime}\right)= \\
& =\sum_{i=0}^{t+1}(-1)^{i}\left(P^{\prime[i+1]}\right)_{V}, V_{t-i+1}^{\prime s}\left(A^{\prime}\right) \text {. }
\end{aligned}
$$

Basta quindi confrontare l'ultimo valore di $R^{\prime}$ con quello fornito dalla (8), per dedurre l'equivalenza

$$
\sum_{i=0}^{t+1}\left[\left(P_{V, i}\right)^{\prime}-(-1)^{i}\left(P^{\prime i+1+1}\right)_{V}\right] \cdot V_{t-i+1}^{\prime s}\left(A^{\prime}\right)=0 .
$$

L'espressione che figura nella (9) entro parentesi quadre è nulla per $i=0$; sicchè, procedendo per induzione rispetto ad $i$ ed utilizzando appunto la (9) (valida per $t=0,1,2, \ldots$ ), si deduce ch'essa si annulla pure per $i=1,2,3, \ldots$. Risulta dunque

talchè :

$$
\left(P_{V, i}\right)^{\prime}=(-1)^{i}\left(P^{\prime i+1]}\right)_{V^{\prime}},
$$

Data una varietà $P$ immersa in $V(p<v-1)$, si trasformi $V$ in $V^{\prime}$ mediante una dilatazione $D$ di base $P$; e sia $P^{\prime} l^{\prime}$ ipersuperficie di $V^{\prime}$ nella quale $D$ dilata la $P$. La successione covariante d'immersione di $P$ in $\nabla$ non $\grave{e}$ allora altro che la trasformata, mediante $D^{-1}$, dell'alternante della successione caratteristica dell' ipersuperficie $P^{\prime}$ in $V^{\prime}$.

Come corollario immediato, da qui si ottiene una nuova dimostrazione della proprietà enunciata nel penultimo capoverso del n. 13 (e già altrimenti stabilita nei nn. 14, 15).

17. Un altro esempio, che poi generalizzeremo, è dato dal seguente teorema.

Le varietà covariantì d'immersione $d^{\prime}$ 'una $P$ che sia intersezione regolare e semplice dis ipersuperficie $A^{1}, A^{2}, \ldots, A^{s} d i V(s=v-p \geq 1)$, si esprimono con le

$$
P_{V, i}=P \cdot \tilde{V}_{i}(A),
$$

dove le $\tilde{V}_{i}$ si calcolano nel modo indicato alla fine del $n .7$. 
Nell'ipotesi che sia $s=1$, questo teorema riducesi a quello del n. 14; potremo quindi supporre $s \geq 2$ ed ammettere il teorema nel caso di $s-1$ ipersuperficie. Definite le $V^{\prime}, A^{\prime \prime}$ mediante le $\left(2_{15}\right)$, in virtù del n. $\left.3, a^{\prime}\right)$ abbiamo :

$$
P=\left(A^{3} A^{2} \ldots A^{s}\right)_{V}=\left(A^{\prime 1} A^{\prime 2} \ldots A^{\prime s-1}\right)_{V^{\prime}},
$$

onde l'ammessa induzione fornisce:

$$
P_{V^{\prime}, j}=\left(\left.P \cdot \tilde{V}_{j}^{\prime}\left(A^{\prime}\right)\right|_{V^{\prime}} .\right.
$$

Basta allora applicare la $\left(9_{15}\right)$, e ricordare il n. 5 , a) ed i nn. 6, 7, per dedurre che è:

$$
\begin{aligned}
P_{V, i} & =\sum_{j=0}^{i}(-1)^{i-j}\left(P \cdot \tilde{V}_{j}^{\prime}\left(A^{\prime}\right)\right)_{V^{\prime}} \cdot V^{\prime[i-j]}= \\
& =\sum_{j=0}^{i}(-1)^{i-j}\left(P \cdot \tilde{V}_{j}^{s-1}(A)\right)_{V} \cdot V^{\prime[i-j]}= \\
& =P \cdot(-1)^{i} \sum_{j=0}^{i} \tilde{\bar{V}}_{j}^{s-1}(A) \cdot V^{\prime[-j]}=P \cdot(-1)^{i} \tilde{\bar{V}}_{i}^{s}(A)=P \cdot \tilde{V}_{i}(A) .
\end{aligned}
$$

Ia successione covariante $\left\{P_{V}\right\}$ della $P$ suddetta, ammette in $\mathfrak{z}_{P}$ un'inversa $\left\{\tilde{P}_{V}\right\} \cdot\left(\right.$ n. 6). Per determinare gli elementi $\tilde{P}_{V, i}$ di quest'ultima, moltiplichiamo ambo i membri della (1) per $\nabla_{j-i}(A)$, e sommiamo per $i=0,1, \ldots, j$ Avuto riguardo al n, 5, a), otteniamo cosi le relazioni

$\left.\sum_{i=0}^{j}\left(P_{V, i} \cdot P V_{j-i}(A)\right)_{P}=\sum_{i=0}^{j} \mid P_{V, i} V_{j-i}(A)\right)_{V}=P \cdot \sum_{i=0}^{j}\left(\tilde{V}_{i}(A) \cdot V_{j-i}(A)=\left\{\begin{array}{l}P \text { se } j=0 . \\ 0 \text { se } j>0,\end{array}\right.\right.$

dalle quali discende (n. 7) che è:

$$
\tilde{P}_{V, i}=P \cdot V_{i}(A) \text {. }
$$

\section{\& III. - Relazioni fra successioni covarianti.}

18. Completiamo la definizione data nel $\& 1$ per la successione $\left\{P_{V}\right\}$ covariante d'immersione di $P$ in $V$, ponendola uguale alla successione unitaria di $\mathfrak{A}_{P}$ nell'ipotesi in cui $V$ coincida con $P$, ossia assumendo:

$$
\left\{P_{P}\right\}=P, 0,0, \ldots \text {. }
$$

Possiamo allora dimostrare il

Teorema DeLL'APPARTENENZA. - Fra le successioni covarianti relative a tre varietà $P, M, V$ tali che sia

$$
P \subset M \subset V
$$

intercede la relazione

$$
\left\{P_{V}\right\}=\left(\left\{P_{M}\right\} \cdot\left|M_{V}\right|\right)_{M} .
$$


Osserviamo anzitutto che, in forza della (1), la (3) è ovviamente verificata nell'ipotesi che $M$ coincida con $P$ o con $V$. Possiamo dunque limitarci al caso in cui le (2) valgano in senso stretto, e cosi risulti

$$
p<m<v \text {. }
$$

Inoltre, in virtù della seconda proposizione in corsivo del n. 8, la (3) equi. vale alla

$$
\left|P_{M}\right|=\left(\left|P_{V}\right| \cdot\left|\tilde{M}_{V}\right|\right)_{M} \text {. }
$$

Ora, nell' ipotesi che $M$ sia un'ipersuperficie di $V$, e ciò̀ se

$$
m=v-1 \text {, }
$$

la $\left(2_{17}\right)$ fornisce:

$$
\tilde{M}_{V, 0}=M, \quad \tilde{M}_{V, 1}=\left(M^{[2]}\right)_{V}, \quad \tilde{M}_{V, i}=0 \quad \text { se } i \geq 2 ;
$$

sicohè, avuto riguardo ai nn. 7 e 5 , a), e tenuta presente la $\left(4_{15}\right)$, la (4) si traduce nelle

$$
P_{M, i}=P_{V, i}+\left(P_{V, i-1} M\right)_{V} \quad(i=0,1,2, \ldots),
$$

le quali attualmente di fatto sussistono, in forza delle $\left(8_{15}\right)$.

Introdotto per una terna (2) il carattere

$$
\pi=v-m,
$$

abbiamo pertanto che il teorema dell'appartenenza sussiste se $\pi=1$. Possiamo quindi supporre $\pi \geq 2$, e procedere per induzione rispetto a $\pi$ ammettendo il teorema per terne aventi quel carattere inferiore a $\pi$. Scelto un qualunque intero $n$ intermedio fra $m$ e $v$, fissiamo comunque in $V$ una varietà $N$ - di dimensione $n$ - passante genericamente per $M$. Potremo allora applicare il teorema dell'appartenenza a ciascuna delle terne

$$
P \subset N \subset V, \quad P \subset M \subset N, \quad M \subset N \subset V,
$$

il che fornisce le

$\left.\left.\left.\left.\left\{P_{V}\right\}=\left(\left|P_{N}\right| \cdot\left|N_{V}\right|\right)_{N}, \quad \mid P_{N}\right\}=\left(\left\{P_{M}|\cdot| M_{N}\right\}\right)_{s t}, \quad \mid M_{V}\right\}=\left(\mid M_{N}\right\} \cdot \mid N_{V}\right\}\right)_{N}$.

Se infine eliminiamo $\left\{P_{N}\right\}$ fra le prime due di queste relazioni, e poi suecessivamente applichiamo la $\left(5_{8}\right)$ e l'ultima di tali relazioni, otteniamo precisamente la (3).

Il teorema dell' appartenenza è così stabilito. In virtù della terz' ultima proposizione del n. 8, dalle (3), (4) si traggono le

(5) $\left.\left.\quad \mid \tilde{P}_{V}\right\}=\left(\left\{\tilde{P}_{M}\right\} \cdot \mid \tilde{M}_{V}\right\}\right)_{M}$,

(6) $\quad\left|\tilde{P}_{M}\right|=\left(\left\{\tilde{P}_{V}\right\} \cdot\left\{M_{V}\right\}\right)_{M}$.

È superfluo aggiungere che, avuto riguardo ai nn. 6,7 , ciascuna delle (3)-(6) equivale a $p$ relazioni non banali fra le varietà delle successioni covarianti che in esse figurano. Così, p. es., la (3) si traduce con le:

$$
P_{V, i}=\sum_{j=0}^{i}\left(P_{M, j} M_{V, i-j}\right)_{M} \quad(i=1,2, \ldots, p) .
$$


19. Ci proponiamo ora di dimostrare il

TeORema DeLLA Sezione. - Se due sottovarietà $P, V^{\prime} d i \quad V$ si segano regolarmente lungo una varietà irriducibile e non singolare, $P^{\prime}$, la sezione con $V^{\prime}$ della successione covariante di $P$ in $V$ coincide con la successione covariante di $P^{\prime}$ in $V^{\prime}$.

Si tratta di far vedere che dalla

seguono le

$$
P^{\prime}=\left(P V^{\prime}\right) v
$$

$$
P^{\prime} V^{\prime}, i=\left(P_{V, i} V^{\prime}\right)_{V} \quad(i=0,1,2, \ldots) .
$$

Ora ̀̀ chiaro, tenuto conto della $\left(5_{13}\right)$, che la (2) sussiste per $i=0$, riducendosi allora alla (1); la (2) ̇̀ inoltre banale se $i>p^{\prime}$, ove - a norma delle solite convenzioni $-p^{\prime}$ denoti la dimensione di $P^{\prime}$, riducendosi in tal caso all' uguaglianza $0=0$. Basteræ̀ dunque, per induzione, provare che la (2) è verificata per un valore $t$ dell'indice $i$ fissato comunque nell'intervallo

$$
1 \leq t \leq p^{\prime},
$$

supponendone già acquisita la validità per $i=0,1, \ldots, t-1$. In corrispondenza ad un $t$ siffatto, la $\left(2_{13}\right)$ fornisce per $s$ un valore $(s=t \dashv v-p)$ che manifestamente soddisfa alle $\left(1_{13}\right)$; sicchè, scelte genericamente $s$ ipersuperficie $\left(3_{13}\right)$ di $V$ passanti per $P$, potremo applicare la $\left(4_{13}\right)$. Posto per abbreviare

$$
A^{\prime i}=\left(A^{\prime} V^{\prime}\right)_{V} \quad(i=1,2, \ldots, s),
$$

se moltiplichiamo ambo i membri della $\left(4_{43}\right)$ per $V^{\prime}$, servendoci dei nn. 5, a') ed 11, otteniamo

$$
\begin{aligned}
\left(A^{\prime 1} A^{\prime 2} \ldots A^{\prime s}\right)_{V^{\prime}}-\left(A^{\prime 1} A^{\prime 2} \ldots A^{\prime s}\right)_{V^{\prime}}^{P^{\prime}} & =\sum_{i=0}^{t}\left(P_{V, i} V_{t-i}^{s}(A) V^{\prime}\right)_{V}= \\
& =\sum_{i=0}^{t}\left[\left(P_{V, i} V^{\prime}\right)_{V} V_{t-i}^{\prime s}\left(A^{\prime}\right)\right]_{V^{\prime}} .
\end{aligned}
$$

D'altro canto, poichè - a norma della (1) - risulta $p^{\prime}=p+v^{\prime}-v$, così quel valore di $s$ soddisfa alle relazioni

$$
v^{\prime}-p^{\prime} \leq s \leq v^{\prime}, \quad t=s+p^{\prime}-v^{\prime},
$$

analoghe alle $\left(1_{13}\right),\left(2_{13}\right)$. Avuto riguardo alle (1), (3), su $V^{\prime}$ (e quindi pure su $V$ ) vale pertanto l'equivalenza

$$
\left(A^{\prime 1} A^{\prime 2} \ldots A^{\prime s}\right)_{V^{\prime}}-\left(A^{\prime 1} A^{\prime 2} \ldots A^{\prime s}\right)_{V^{\prime}}^{P^{\prime}}=\sum_{i=0}^{t}\left(P_{V^{\prime}, i}^{\prime} V_{t-i}^{\prime s}\left(A^{\prime}\right)\right)_{V^{\prime}},
$$

che si deduce dalla $\left(4_{13}\right)$ accentuando tutte le lettere. Basta ora confrontare le altime due equivalenze, tenendo conto dell'ammessa induzione, per dedurne la

$$
0=\sum_{i=0}^{t}\left[\left(P_{V^{\prime}, i}^{\prime}-\left(P_{V, i} V^{\prime}\right)_{V}\right) V_{t-i}^{\prime s}\left(A^{\prime}\right)\right]_{V^{\prime}}=P_{V^{\prime}, t}^{\prime}-\left(P_{V, t} V^{\prime}\right)_{V}
$$


La (2) è così dimostrata per $i=t$, e quindi per ogni valore di $i$. Applicando ad essa un'argomentazione analoga a quella che, alla fine del n. 17, ci ha permesso di derivare le $\left(2_{17}\right)$ dalle $\left(1_{17}\right)$, si ottiene che dalle (1) seguono le (4)

$$
\tilde{P}_{V^{\prime}, i}^{\prime}=\left(\tilde{P}_{V, i} V^{\prime}\right)_{V} \quad(i=0,1,2, \ldots) \text {. }
$$

20. Stabiliremo infine il

TEOREMA DEL PRODOTTO. - La successione covariante in $V$ del prodotto di due o più sottovarietà di $V$, coincide col prodotto in $\mathfrak{A}_{V}$ delle successioni covarianti relative ai singoli fattori.

Incominciamo dal caso in cui il prodotto abbia due fattori. Si tratta allord di dimostrare che la $P=M N$ implica la $\{P\}=|M| \cdot \mid N\}$, ossia - più specificamente - che dalla

$$
P=(M N)_{V}
$$

segue la

$$
\left.\left|P_{V}\right|=\left(\mid M_{V}\right\} \cdot \mid N_{V}\right\}_{V} \text {. }
$$

All' uopo si osservi che (n. 4) - in virtù della (1) - valgono le $\left(2_{18}\right)$, e quindi pure la $\left(3_{18}\right)$ :

$$
\left.\left|P_{V}\right|=\left(\left\{P_{M}\right\} \cdot \mid M_{V}\right\}\right)_{M} \text {. }
$$

Inoltre, in base al teorema della sezione (n. 19), dalla (1) si trae la

$$
\left|P_{M}\right|=\left(\left|N_{V}\right| M\right)_{V}
$$

Risulta pertanto

$$
\left|P_{V}\right|=\left(\left\{M_{V}\right\} \cdot\left(\mid N_{V}\{M)_{V}\right)_{M}\right.
$$

e da qui si deduce subito la (2), applicando la proposizione finale del n. 8 . Dimostreremo ora per induzione il teorema nel caso di un prodotto

$$
Q=\left(M^{1} M^{2} \ldots M^{s}\right)_{V}
$$

di $s \geq 3$ fattori, supponendolo già acquisito per prodotti di $s-1$ fattori. Posto

$$
N=\left(M^{2} \ldots M^{s}\right)_{V},
$$

avremo dunque

$$
\left.\left\{N_{V}\right\}=\left(\left|M_{V}^{2}\right| \ldots \mid M_{V}^{s}\right\}\right)_{V} .
$$

D'altronde le (3), (4) porgono:

$$
Q=\left(M^{1} N\right)_{V}
$$

sicchè, per il caso del teorema già acquisito, risulta

$$
\left\{Q_{V}\right\}=\left(\left\{M_{V}^{1}\right\} \cdot\left\{N_{V}\right\}\right)_{V} .
$$


Non v'è quindi che da eliminare $\left\{N_{V}\right\}$ fra le (5), (6), per ottenere la formula richiesta :

$$
\left.\left.\left|Q_{V}\right|=\left(\mid M_{V}^{1}\right\} \cdot \mid M_{V}^{2}\right\} \ldots\left|M_{V}^{s}\right|\right)_{V} .
$$

Rileviamo poi che basta applicare la penultima proposizione del n. 8 alle (2), (7), per dedurre che:

Le (1), (3) rispettivamente implicano le

$$
\left.\left.\left\{\tilde{P}_{V}\right\}=\left(\mid \tilde{M}_{V}\right\} \cdot\left\{\tilde{N}_{V}\right\}\right)_{V}, \quad(9) \quad\left\{\tilde{P}_{V}\right\}=\left(\left\{\tilde{M}_{V}^{\mathrm{i}}\right\} \cdot\left\{\tilde{M}_{V}^{2}\right\} \ldots \mid \tilde{M}_{V}^{s}\right\}\right)_{V}
$$

Questo risultato permette, fra l'altro, di riottenere la proposizione àel n. 17 come conseguenza immediata di quella del n. 14.

\section{§ IV. - Dipendenze fra varietà di una successione covariante.}

21. Siano $P$ una varietà irriducibile non singolare (di dimensione $p$ ) $\Theta$ $V^{\prime}$ un'ipersuperficie di $V$, tali che

$$
P \subset V^{\prime} \subset V \text {. }
$$

Posto per abbreviare

$$
P^{\prime}=\left(P V^{\prime}\right)_{V}
$$

$$
W=\left(V^{\prime}[?]\right)_{V},
$$

dalle (1), (2) discende (n. 4) che è :

$$
P^{\prime} \subset W \subset V
$$

inoltre dalla (3), poggiando sul n. 17, si traggono le:

$$
W_{V, h}=(-1)^{h}(h+1)\left(W V^{\prime[h]}\right)_{V} \quad(h=0,1,2, \ldots) .
$$

Ci proponiamo di dimostrare che,

Con le precedenti notazioni, risulta:

$$
\tilde{P}_{V, i}=\tilde{P}_{V^{\prime}, i}+\tilde{P}_{W, i-1}^{\prime} \quad(i=0,1,2, \ldots) .
$$

Osserviamo anzitutto che, poichè - a normà della (2) $-P^{\prime}$ ha dimensione $p^{\prime}=p-1$, così ciascuna delle varietà che compaiono nella (6) ha la dimensione $p-i$. La (6) ̀े quindi soddisfatta (e banalę) se $i>p$, e lo è pure per $i=0$, in forza delle $\left(5_{13}\right),\left(4_{15}\right)$; basterà dunque dimostrarla nell'ipotesi che sia $i=1,2, \ldots, p$. A tal nopo, applichiamo alla terna (1) il teorema dell'appartenenza sotto la forma espressa dalla seconda delle $\left(5_{18}\right)$ (nella quale ora va scritto $V^{\prime}$ in luogo di $M$ ), ciò che fornisce le:

$$
\tilde{P}_{V^{\prime}, i}=\sum_{j=0}^{i}\left(\tilde{P}_{V, j} V^{\prime}{ }_{V, i-j}\right)_{V} \cdot
$$

Poichè - in virtù del n. $14-$ si ha

$$
V^{\prime}{ }_{V, i-j}=(-1)^{i-j}\left(V^{\prime} V^{\prime[i-j]}\right)_{V},
$$


cosi, tenuto conto del n. 5, a), l'ultima relazione diventa

ossia:

$$
\tilde{P}_{V^{\prime}, i}=\sum_{j=0}^{i}(-1)^{i-j}\left(\tilde{P}_{V, j} V^{\prime[i-j]}\right)_{V}
$$

$$
\tilde{P}_{V^{\prime}, i}=\tilde{P}_{V, i}+\sum_{j=0}^{i-1}(-1)^{i-j}\left(\tilde{P}_{V, j} V^{\prime[i-j]}\right)_{V} .
$$

Applicando quindi alla terna (4) il teorema dell'appartenenza, ancora sotto la forma suddetta, otteniamo

$$
\tilde{P}_{W, i-1}^{\prime}=\sum_{j=0}^{i-1}\left(\tilde{P}_{V, i}^{\prime} W_{V, i-j-1}\right)_{W}
$$

E questa relazione, avuto riguardo alle (5) ed al n. 5, a), può scriversi nella forma :

$$
\tilde{P}_{W, i-1}^{\prime}=-\sum_{j=0}^{i-1}(-1)^{i-j}(i-j)\left(\tilde{P}_{V, j}^{\prime} \nabla^{\prime i-j-1]}\right)_{V}
$$

Infine dalla (2), usufruendo del teorema del prodotto sotto la forma $\left(8_{20}\right)$, si traggono le

$$
\tilde{P}_{V, j}^{\prime}=\sum_{h=0}^{j}\left(\tilde{P}_{V, j-h} \tilde{V}_{V, h}^{\prime}\right)_{V}
$$

e cioes, essendo (n. 14): $\left|\tilde{V}_{V}^{\prime}\right|=V^{\prime}, \nabla^{\prime 2]}, 0,0, \ldots$, le

$$
\tilde{P}_{V, j}^{\prime}=\left(\tilde{P}_{V, j} V^{\prime}\right)_{V}+\left(\tilde{P}_{V, j-1} V^{\prime 2]}\right)_{V} .
$$

Sostituendo nella (8), dopo facili riduzioni si ottiene

$$
P^{\prime} W, i-1=-\sum_{j=0}^{i-1}(-1)^{i-i}\left(\tilde{P}_{V, j} V^{\prime i-j]}\right)_{V}
$$

Basta quindi sommare a membro a membro questa relazione con la (7) per ricavare la $(6)$.

22. Se $P \subset V$, le varieta $P_{V, i}$ risultano - eome vedremo - fra loro legat $\theta$ in $\mathfrak{F}_{V}$ non appena la dimensione $p$ di $P$ sia abbastanza grande rispetto alla dimensione $v$ di $V$. Cosi, ad esempio se $P$ è un'ipersuperficie di $V$, in base al n. 14 risulta:

$$
\tilde{P}_{V, 1}=\left(P^{[2]}\right)_{V}, \quad \tilde{P}_{V, i}=0 \quad \text { per } \quad i \geq 2 \quad(p=v-1) .
$$

Ci proponiamo, più generalmente, di dimostrare che:

Nell' ipotesi che sia $p>v / 2$, si ha

$$
\tilde{P}_{V, i}=0
$$

per ogni $i$ soddisfacente alle

$$
v-\boldsymbol{p}<i \leq p
$$


Introdotto all' nopo il carattere

$$
\pi=v-p
$$

relativo alla coppia $P, V$ (con $P \subset V$ ), abbiamo intanto, in virtù di ciò che precede, che il risultato enunciato sussiste se $\pi=1$. Possiamo quindi supporre $\pi \geq 2$, ed ammettere induttivamente quel risultato per valori di detto carattere inferiori a $\pi$. Scelta genericamente in $V$ un'ipersuperficie $V^{\prime}$ passante per $P$, definiamo le varietà $P^{\prime}, W$ mediante le $\left(2_{21}\right),\left(3_{21}\right)$ (talchè $P^{\prime} \subset W$ ), ed osserviamo che la coppia da queste formata ha il carattere

$$
\pi^{\prime}=w-p^{\prime}=(v-2)-(p-1)=v-p-1<\pi .
$$

Ne consegue che, per ogni $i$ che soddisfi alle $w-p^{\prime}<i-1 \leq p^{\prime}$, e cioè alle (2), risulta

$$
\tilde{P}_{W, i-1}^{\prime}=0 \text {. }
$$

Ragionando similmente sulla coppia $P, V^{\prime}$ (con $P \subset V^{\prime}$, e col carattere $\left.\pi_{1}=v^{\prime}-p=(v-1)-p<\pi\right)$, vediamo che dev' essere

$$
\tilde{P}_{V^{\prime}, i}=0
$$

per ogni $i$ soddisfacente alle

$$
v-p-1<i \leq p,
$$

e quindi a fortiori per ogni $i$ che soddisfi alle (2).

Per ogni $i$ siffatto sussistono quindi le (3), (4), dalle quali si ricava tosto la (1) applicando la $\left(6_{21}\right)$.

23. Proveremo da ultimo che:

Conservando le notazioni del numero precedente, la prima varietà caralteristica di $P$ in $V$ equivale alla $\tilde{P}_{V, v-p}$, ossia sempre risulta

$$
\tilde{P}_{V, v-p}=\left(P^{[2]}\right)_{V} \text {. }
$$

La (1) $\partial$ soddisfatta in modo banale se $p=v$, ed anche se $v>2 p$ in quanto allora la dimensione virtuale $2 p-v$ di ciascuno dei suoi due membri risulta negativa. Basterà dunque stabilirla nell'ipotesi.che sia

$$
1 \leq v-p \leq p \text {. }
$$

In virtù del primo capoverso del n. 22, e con le notazioni del n. 22, la (1) sussiste se il carattere $\pi=v-p$ ha il valore 1. Potremo quindi supporre $\pi \geq 2$ e, procedendo per induzione completa, applicare il risultato enunciato alla coppia $P^{\prime}, W$ (la quale ha quel carattere espresso da $\pi^{\prime}=v-$ $-p-1<\pi)$. Otteniamo cosi intanto :

$$
\tilde{P}_{W, v-p-1}^{\prime}=\left(P^{\prime[2]}\right)_{W} \text {. }
$$


Osserviamo, in secondo luogo, che per $i=v-p$ sono soddisfatte le $\left(5_{22}\right)$ e quindi pure (n. 22, la $\left(4_{22}\right)$. Pertanto dalla $\left(6_{21}\right)$ si trae la:

$$
\tilde{P}_{V, v-p}=\tilde{P}_{W, v-p-1}^{\prime} \text {. }
$$

Da ultimo, avuto riguardo alle $\left(1_{21}\right),\left(2_{21}\right),\left(3_{24}\right)$ ed applicando il n. $\left.5, b^{\prime}\right)$, otteniamo

$$
\left(P^{\prime 21}\right)_{W}=\left(P^{[2]}\right)_{V}
$$

Basta allora sommare questa relazione a membro a membro con le (2), (3), per dedurne la (1).

\section{$\S$ V. - Costruzione di varietà covarianti operando entro anelli di $V$.}

23'. Le varietà $P_{V, i}$ - covarianti d'immersione di $P$ in $V$ - sono state introdotte nel $n .13$ poggiando sulla $\left(4_{13}\right)$, e quindi mediante un procedimento che fa intervenire le operazioni su $V$, a tre o più termini, considerate nel $\S$ III del Capitolo primo. L'altra definizione possibile ottenuta nel n. $16^{\prime}$ per le $P_{V, i}$ non presenta più questo inconveniente, ma esige operazioni sopra un'altra varieta $V^{\prime}$ (dedotta da $V$ con una dilatazione di base $P$ ). Nel presente paragrafo mostreremo che, per costruire le $P_{V, i}=P_{i}$, basta effettuare opportune operazioni entro gli anelli di $V$. Cio permetterebbe di definire le $P_{V, i}$ anche per varietà $P$ singolari o spezzate; ma non ci intratterremo qui su tali applicazioni.

Vedremo poi, nel Capitolo successivo, come sia possibile di esprimere le suddette operazioni a tre o più termini facendo intervenire le varietà covariantì delle sottovarietà di $V$ fra cui esse operano; ciò permetterà - in ultima analisi - di ridurre tali operazioni ad operazioni entro gli anelli di $V$. Avremo cosi modo di risolvere nel senso più largo, sia funzionalmente che numerativamente, vari problemi (regolari od irregolari) d'intersezione sopra $V$.

24. Avuto riguardo alla $\left(5_{13}\right)$, potremo stabilire quanto enunciato nel primo capoverso del n. 23', supponendo $i>0$ e procedendo per induzione rispetto ad $i$. Ammetteremo quindi di aver già determinate sulle $P$ di $V$ le varietà covarianti $P_{0}, P_{1}, \ldots, P_{i-1} ;$ e da queste, giusta il n. 7 , si potranno subito dedurre le $\tilde{P}_{0}, \tilde{P}_{1}, \ldots, \tilde{P}_{i-1}$ mediante operazioni dell'anello $\mathfrak{A}_{P}$. Si tratta ora di costruire $P$, operando entro gli anelli di $V$. All'uopo distinguiamo tre casi, secondochè $i$ risulta uguale, maggiore o minore della differenza $v-p$ : ed osserviamo che, in ogni easo, è lecito supporre $i \leq p$, poichè altrimenti si ha $P_{i}=0$ e non v'è altro da aggiungere.

1) Se $i=v-p \leq p$, possiamo applicare la $\left(1_{23}\right)$, in virtù della quale la formula data dalla $\left(2_{\gamma}\right)$ per tale valore di $i$ fornisce $\operatorname{la}$ :

$$
P_{v-p}=-\left(P^{[2]}\right)_{V}-\sum_{j=1}^{v-p-1}\left(P_{j} \tilde{P}_{v-p-j}\right)_{P}
$$

che porge per $P_{i}=P_{v-p}$ una costruzione del tipo voluto. 
2) Se $v-p<i \leq p$, possiamo applicare la $\left(1_{2 z}\right)$, valida per quell' $i$ e per tutti i valori di $i$ soddisfacenti alle $\left(2_{22}\right)$. Tenuto conto di cio, dalla $\left(2_{7}\right)$ si trae la :

$$
P_{i}=-\sum_{j=1}^{v-p}\left(P_{i-j} \tilde{P}_{j}\right)_{P}
$$

la quale porge per $P_{i}$ una costruzione del tipo richiesto.

3) Se $i<v-p$, il numero

$$
s=v-p-i
$$

risulta positivo. Prese in $V$ ad arbitrio $s$ ipersuperficie

$$
A^{1}, A^{2}, \ldots, A^{s}
$$

passanti genericamente per $P$, la loro intersezione è una varietà

di dimensione

$$
M=\left(A^{1} A^{2} \ldots A^{s}\right)_{V}
$$

$$
m=v-s=p+i,
$$

passante semplicemente per $P$. La varietà $P$ presenta il caso 1) relativamente ad $M$, sicchè - a norma di quanto ottenuto in 1) - è lecito supporre di aver determinato la varietà covariante d'immersione $P_{M, i}$. Se ora applichiamo alla terna $P \subset M \subset V$ il teorema dell' appartenenza nella forma espressa dalla $\left(4_{48}\right)$, determinandovi $\left\{\tilde{M}_{V}\right\}$ in base al n. 16, otteniamo:

$$
P_{i}=P_{M, i}-\sum_{j=1}^{i}\left(P_{i-j} V_{j}(A)\right)_{V}
$$

Questa formula porge per $P_{i}$ una costruzione del tipo voluto: si potrebbe dimostrare direttamente che la varietà $P_{i}$ da essa definita non dipende dalla scelta delle ipersuperficie $A$, il che però è già chiaro a priori in base al $\$ 1$ del presente Capitolo.

25. Le considerazioni dei due numeri precedenti suggeriscono il pro. blema generale che ora enunceremo.

Siano date in $V$ due o più varietà $M^{1}, M^{2}, \ldots, M^{s}$, dalle quali si deduca una varietà $N$ operando fra esse in modo determinato entro gli anelli $\mathfrak{A}_{M}$ ed $\mathfrak{H}_{V}$; si tratta di esprimere le varietà covarianti d'immersione di $N$ in $V$, in funzione delle varietà covarianti delle $M$, mostrando come le prime possano ottenersi dalle seconde operando tra esse entro gli anelli suddetti.

Esempi in proposito abbiamo già dati nei nn. 17-20, trattando questioni abbastanza generali del tipo indicato, per le quali la risposta si presenta re. lativamente semplice dal punto di vista formale. Una trattazione esauriente di quel problema porterebbe però a difficoltà algoritmiche assai gravi, come già apparirà dal caso elementare - a cui ci limiteremo - in cui le $M$ siano 
ipersuperficie di $V$ ed $N$ sia la loro somma; vedremo infatti che in tal caso il problema indicato si risolve effettuando soltanto operazioni in $\mathfrak{A}_{V}$, ma che la determinazione esplicita di queste non è esente da complicazioni formali.

Incominciamo all' nopo col richiamare i risultati del n. 17. Se $A^{\prime}$, $A^{2}, \ldots, A^{s}$ sono $s$ ipersuperficie di $V$, $\Theta$ se $P=A^{1} A^{2} \ldots A^{s}$ denota il loro prodotto in $\mathfrak{Z}_{V}$ (di dimensione $p=v-s \geq 0$ ), la successione covariante di $P$ in $V$ ha come alternante quella formata dalle varieta

$$
\bar{P}_{i}=P \cdot \tilde{\bar{V}}_{i}{ }^{8} \quad(i=0,1,2, \ldots),
$$

dove (n. 7) $\tilde{\bar{V}}_{\mathrm{o}}=V$ e (per $\left.i \geq 1\right) \tilde{\bar{V}}_{i}^{s}$ denota la somma di tutti gli $\left(\begin{array}{c}s+i-1 \\ i\end{array}\right)$ prodotti delle $A^{1}, A^{2}, \ldots, A^{s}$ prese con ripetizione ad $i$ ad $i$. Questo risultato conserva la sua validità nel caso in cui fra le $A$ vi siano delle equivalenze, e può quindi venir utilizzato per determinare, più generalmente, le varietà $\bar{M}_{i}$ relative ad un qualunque monomio $M$ del tipo

$$
M=\left(A^{1}\right)^{\left[k_{1}\right]}\left(A^{z}\right)^{\left[k_{2}\right]} \ldots\left(A^{s}\right)^{\left[k_{s}\right]} \quad\left(k_{1} \geq 0, k_{2} \geq 0, \ldots, k_{s} \geq 0\right) .
$$

In vista di future applicazioni, osserviamo che, in base alla definizione delle $\tilde{\bar{V}}_{i}^{s}$, risulta

$$
\tilde{\bar{V}}_{i}^{s}=\tilde{\bar{V}}_{i}^{s-1}+A^{s} \tilde{\bar{V}}_{i-1}^{s} \quad\left(\tilde{\bar{V}}_{-1}^{s}=0\right)
$$

Dalla (1) si ha quindi che

Se $B$ denota una qualunque ipersuperficie di $V$ ed inoltre, come dianzi, assumiamo $P=A^{1} A^{2} \ldots A^{s}$, allora:

$$
(\overline{B P})_{t}=B\left(\bar{P}_{t}+(\overline{B P})_{t-1}\right)
$$

ov' è soltinteso che tutti $i$ prodotti vanno effeltuali $s u \quad V$.

Riferendoci ancora al monomio $M$ dato dalla (2), e denotandone con $k=k_{1}+k_{z}+\ldots+k_{s}$ il grado (talchè $M$ risulta una varietà di dimensione $v-k$, uguale allo zero di $\mathfrak{A}_{V}$ se $k>v$ ), sarà comodo per il seguito porre

$$
[M]_{|i|}=\bar{M}_{i-k+s} \text {, }
$$

dove il secondo membro si calcola nel modo anzidetto, ed ̀े quindi una varietà di dimensione $v-i-1$, da porsi uguale allo zero di $\mathfrak{A}_{V}$ se $i \geq v$ o se $k>i+1$. Più in generale, consideriamo una qualunque serie formale di potenze nelle $A$ (a coefficienti $c$ interi):

$$
f(A)=\Sigma c\left(A^{i}\right)^{\left[k_{1}\right]}\left(A^{z}\right)^{\left[k_{2}\right]} \ldots\left(A^{s}\right)^{\left[k_{s}\right]}=\Sigma c M,
$$

la quale potrà però sempre venir ridotta ad un polinomio (non necessariamente omogeneo) nelle $A$, col sopprimere tutti i monomi di grado $k>v$, d'accordo col fatto che ciascuno di questi, come elemento di $\mathfrak{A}_{V}$, risulta uguale allo zero (n. 3). Assumeremo allora, per definizione,

$$
[f(A)]_{i \mid}=\Sigma c[M]_{\{i\}} \quad(i=0,1, \ldots, v-1)
$$


il che val quanto dire che conveniamo che l'operazione $[. . .]_{\{i\}}$ sia distributiva rispetto alla somma e tale che risulti

$$
[c M]_{\{i\}}=c[M]_{\{i\}} .
$$

Si noti che il secondo membro della penultima equazione risulta in ogni caso un polinomio omogeneo di grado $i+1$ nelle $A$, rappresentante una varietà pura - di dimensione $v-i-1-$ di $V$.

Cio premesso, siamo in grado di scrivere la formula rispondente alla questione propostaci. Consideriamo le funzioni simmetriche elementari delle $A$ :

$$
V_{i}=\Sigma A^{1} A^{2} \ldots A^{i} \quad(i=1,2, \ldots, s),
$$

e denotiamo con $f(A)$ la serie formale di potenze nelle $A$ (a coefficienti interi non negativi) che si ottiene sviluppando in serie la funzione razionale delle $A$ data dal secondo membro della

$$
f(A)=\frac{V_{1}+2 V_{2}+\ldots+s V_{s}}{1-V_{2}-2 V_{3}-\ldots-(s-1) V_{s}} .
$$

Proveremo che le varietà covarianti dell' ipersuperficie C somma delle A:

$$
C=A^{1}+A^{2}+\ldots+A^{s}
$$

prese con segno opportuno, si esprimono come somme di varietà covarianti di prodotti delle $A$, mediante la formula

$$
\bar{C}_{i}=[f(A)]_{\{i\}} \quad(i=0,1,2, \ldots) .
$$

A questo risultato si può dare forma più semplice ed esplicita; facendo intervenire anche a secondo membro l'ipersuperficie $C$. Si ottiene così, come vedremo, la formula:

$$
\begin{aligned}
\bar{C}_{i} & =\Sigma{\overline{A^{1}}}_{i}+2 \Sigma\left(\overline{A^{1} A^{2}}\right)_{i-1}+\ldots+s\left(\overline{A^{1} \ldots A^{s}}\right)_{i-s+1}+ \\
& +\Sigma\left(\overline{C A^{1} A^{2}}\right)_{i-2}+2 \Sigma\left(\overline{C A^{1} A^{2} A^{3}}\right)_{i-3}+\ldots+(s-1)\left(\overline{C A^{1} \ldots A^{s}}\right)_{i-s},
\end{aligned}
$$

dove le somme a secondo membro vanno estese alle combinazioni semplici delle $A$ ad una ad una, a due a due, ecc.

Generalizzeremo inoltre la (8) nel modo seguente. Consideriamo un qualunque monomio $Q$ di grado $h$, del tipo (2), i cui fattori possano anche essere ipersuperficie distinte dalle $A$, e denotiamo con $r$ un intero non negativo arbitrario. Proveremo allora la formula

$$
\left(\overline{Q C^{[r]}}\right)_{i}=\left[Q f(A)^{[r]}\right]_{\left\{i^{\prime}\right\}} \text { ove } i^{\prime}=i+h+r-1
$$

si noti che questa include di fatto la (8) per $h=0, r=1$, e si riduce (con altre notazioni) alla (4) per $r=0$. 
26. Ci proponiamo anzitutto di stabilire $i$ risultati precedentemente enunciati, nel caso più semplice $s=2$. Attualmente la $\left(7_{* s}\right)$ si riduoe a

$$
C=A^{1}+A^{2}
$$

e la (9) diventa :

$$
\bar{C}_{\iota}={\overline{A^{1}}}_{i}+{\overline{A^{2}}}_{i}+2\left(\overline{A^{1} A^{2}}\right)_{i-1}+\left(\overline{C A^{1} A^{2}}\right)_{i-2} \quad(i=0,1,2, \ldots),
$$

dove, naturalmente, $i$ termini con indice negativo vanno posti uguali allo zero. La (2) è pertanto soddisfatta per $i=0$, riducendosi allora alla (1). Basterà dunque provarla supponendo $i>0$ ed ammettendo, per induzione, di aver già dimostrato l'eguaglianza

$$
\bar{C}_{i-1}=\bar{A}_{i-1}^{1}+\bar{A}_{i-1}^{2}+2\left(\overline{A^{1} A^{2}}\right)_{i-2}+\left(\overline{C A^{1} A^{1}}\right)_{i-3} \text {. }
$$

All' uopo non v'è che da osservare come la (2) si ottenga moltiplicando fra loro a membro a membro le (1), (3), tenendo presente che, in forza della $\left(3_{85}\right)$, risulta:

$$
\begin{gathered}
C \cdot \bar{C}_{i-1}=\bar{C}_{i}, \quad A^{1} \cdot{\overline{A^{1}}}_{i-1}=\bar{A}^{1}{ }_{i}, \quad A^{2} \cdot \bar{A}_{i-1}=\bar{A}_{i}^{2}, \\
A^{1} \cdot{\overline{A^{2}}}_{i-1}+A^{1} \cdot\left(\overline{A^{1} A^{2}}\right)_{i-2}=\left(\overline{A^{1} A^{2}}\right)_{i-1}, \quad A^{2} \cdot \bar{A}_{i-1}^{1}+A^{2} \cdot\left(\overline{A^{1} A^{2}}\right)_{i-2}=\left(\overline{A^{1} A^{2}}\right)_{i-1}, \\
C\left(\left(\overline{A^{1} A^{2}}\right)_{i-2}+\left(\overline{C A^{1} A^{2}}\right)_{i-2}\right)=\left(\overline{\left.C A^{1} A^{2}\right)_{i-2} .}\right.
\end{gathered}
$$

In secondo luogo, estenderemo la (2) nel modo seguente. Consideriamo in $V$ un qualunque numero $h \geq 0$ d' ipersuperficie $B^{1}, B^{2}, \ldots, B^{h}$, e poniamo

$$
Q=\left(B^{1} B^{z} \ldots B^{h}\right)_{V},
$$

talchè $Q$ sarà una varietà di dimensione $v-h$, da assumersi coincidente con $V$ per $h=0$. Proveremo che è :

$$
(\overline{C Q})_{i}=\left(\overline{A^{1} Q}\right)_{i}+\left(\overline{A^{2} Q}\right)_{i}+2\left(\overline{A^{1} A^{2} Q}\right)_{i-1}+\left(\overline{C A^{1} A^{2} Q}\right)_{i-2},
$$

con la solita convenzione per i simboli ad indice negativo.

A tal fine, osserviamo che la (5) si riduce alla (2) se $h=0$ e che, per $i=0$ ed $h$ qualunque, essa coincide con l'equazione che si ricava dalla (1) moltiplicandone i due membri per $Q$. Potremo dunque supporre $h \geq 1, i \geq 1 \mathrm{e}$ procedere per doppia induzione rispetto ad $h$ ed $i$. Posto per abbreviare

$$
P=\left(P^{1} B^{2} \ldots B^{n-1}\right)_{V}, \quad B^{h}=B,
$$

talchè la (4) può anche scriversi nella forma

$$
Q=B P \text {, }
$$

basterà dunque stabilire la (5) nell'ipotesi di aver già dimostrate le:

$$
\begin{aligned}
& (\overline{C P})_{i}=\left(\overline{A^{1} P}\right)_{i}+\left(\overline{A^{2} P}\right)_{i}+2\left(\overline{A^{1} A^{2} P}\right)_{i-1}+\left(\overline{C A^{1} A^{2} P}\right)_{i-2}, \\
& \left(\overline{C Q} \bar{Q}_{i-1}=\left(\overline{A^{1} Q}\right)_{i-1}+\left(\overline{A^{2} Q}\right)_{i-1}+2\left(\overline{A^{1} A^{2} Q}\right)_{i-2}+\left(\overline{C A^{1} A^{2} Q}\right)_{i-3} .\right.
\end{aligned}
$$


Ora infatti, avuto riguardo alle $(6),\left(3_{25}\right)$, si ottiene la (5) col sommare a membro a membro le (7), (8) - riunendo i termini che in esse appaiono scritti in colonna - e moltiplicare quindi per $B$ ambo, i membri dell'equazione risultante.

La (5) è così dimostrata. Essa, in particolare, fornisce le:

$$
\begin{aligned}
& \left.\left.\left.\left(\overline{C A^{1} A^{2}}\right)_{i-2}=\left(\overline{A^{1}\left(A^{1} A^{2}\right.}\right)\right)_{i-2}+\left(\overline{A^{2}\left(A^{1} A^{2}\right.}\right)\right)_{i-2}+2\left(\overline{A^{1} A^{2}\left(A^{1} A^{2}\right.}\right)\right)_{i-3}+\left(\overline{C\left(A^{1} A^{2}\right)^{[2]}}\right)_{i-4},
\end{aligned}
$$

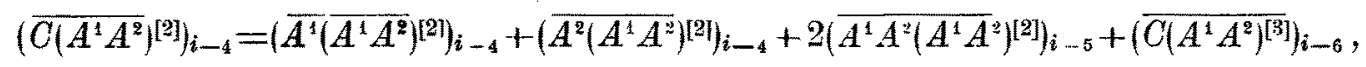

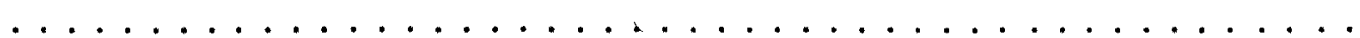

E si noti che, in virtù della convenzione richiamata concernente $\mathrm{i}$ termini ad indice negativo, la successione delle formule così ottenute che non si riducano all' uguaglianza banale $0=0$ risulta $f$ in ita Sommando queste formule $e$ la (2) fra loro a membro a membro, e sopprimendo i termini che vengono a comparire due volte a destra ed a sinistra del segno di uguaglianza, si ottiene una relazione che, con le notazioni del n. 25, può essere scritta in modo compatto sotto la forma:

$$
\bar{C}_{i}=\left[\frac{A^{1}+A^{2}+2 A^{1} A^{2}}{1-A^{1} A^{2}}\right]_{\{i\}} .
$$

La frazione che qui figura entro parentesi quadre è ciò che diventa la funzione $f(A)$, definita dalla $\left(6_{z_{5}}\right)$, nel presente caso $s=2$. Pertanto la (9) dimostra la $\left(8_{2_{5}}\right)$ per $s=2$.

27. Proveremo ora come, in ogni caso, dalla $\left(8_{25}\right)$ possa dedursi la $\left(10_{25}\right)$ : poichè $(n .26)$ la $\left(8_{25}\right)$ è gia stata stabilita per $s=2$, così anche la $\left(10_{25}\right)$ ne seguirà per $s=2$.

Scriviamo la $f(A)$, come nel n. 25, sotto la forma :

$$
f(A)=\Sigma c M
$$

dove $c$ denoti un intero ed $M$ sia un monomio di grado $k$ nelle $A$ con coefficiente unitario. Allora la $\left(8_{25}\right)$ non è che nn modo compatto per esprimere la:

$$
\bar{C}_{i}=\Sigma c \bar{M}_{i-k+1} \text {. }
$$

Orbene, allo stesso modo come nel n. 26 abbiamo dedotto la $\left(5_{26}\right)$ dalla $\left(2_{26}\right)$ |mediante doppia induzione rispetto agli interi $i$ e $h$, e facendo uso della $\left(3_{25}\right)$, così dalla (2) segue la

$$
(\overline{Q C})_{i}=\mathrm{\Sigma} c(\overline{Q M})_{i-k+1},
$$

dove $Q$ denoti un qualunque monomio $\left(4_{26}\right)$, di grado $h$. Poichè il monomio $Q M$ è di grado $h+k$, così, a norma delle convenzioni fatte nel n. 25, risulta

$$
(Q \bar{M})_{i-k+1}=[Q M]_{\left\{i^{\prime}\right\}},
$$


dove, per abbreviare, si ̀̀ posto

$$
i^{\prime}=i+h \text {. }
$$

Avuto riguardo alla (1), si ha pertanto che la (3) equivale alla

$$
(\overline{Q C})_{i}=[Q f(A)]_{i, j}
$$

sicchè è dimostrato che, nell'ipotesi in cui $r=1$ ed $h$ sia qualunque, la $\left(10_{25}\right)$ risulta di fatto una conseguenza della $\left(8_{25}\right)$.

Proveremo quanto asserito al principio di questo numero anche per $r>1$, procedendo per induzione rispetto ad $r$, e quindi ammettendo che valga la formula fornita dalla $\left(10_{25}\right)$ col porvi $r-1$ in luogo di $r$. Elevando la serie formale di potenze (1) alla potenza $(r-1)$-ma, si ottenga la

$$
f^{\prime}(A)=\Sigma c^{\prime} M^{\prime},
$$

ove $c^{\prime}$ denoti un intero ed $M^{\prime}$ sia un monomio di grado $k^{\prime}$ nelle $A^{\prime}$ con coefficiente unitario; sarà allora

$$
f(A)^{[r]}=f(A) \cdot f^{\prime}(A)=\Sigma \Sigma c c^{\prime} M M^{\prime} .
$$

Ciò premesso, per l'ammessa induzione, tenuto conto della $\left(4_{25}\right)$, e rilevato che ̀े $i+(h+1)+(r-1)-1=i^{\prime}$, abbiamo:

$$
\begin{aligned}
\left(\overline{\left.Q \cdot C^{[r]}\right)_{i}}\right. & =\left(\overline{Q C \cdot C^{[r-1}}\right)_{i}=\left[Q C \cdot f^{\prime}(A)\right]_{\left\{i^{\prime}\right\}}= \\
& =\Sigma c^{\prime}\left[Q M^{\prime} C\right]_{\left\{i^{\prime}\right\}}=\Sigma c^{\prime}\left(\overline{Q M^{\prime} C}\right)_{i^{\prime}-\left(h+k^{\prime}+1\right)+1} .
\end{aligned}
$$

D'altro canto, posto per abbreviare

$$
i_{1}=i^{\prime}-h-k^{\prime}=i-k^{\prime}+r-1,
$$

in forza della (1) e della formula che si deduce dalla $\left(10_{25}\right)$ ponendovi rispettivamente $1, i_{1}, Q M^{\prime}$ in luogo di $r, i, Q$, formula che sussiste in virtù del caso $(r=1)$ gia stabilito, risulta :

$$
\left(\overline{Q M^{\prime} \cdot C}\right)_{i_{1}}=\left[Q M^{\prime} \cdot f(A)\right]_{\left\{i^{\prime}\right\}}=\Sigma c\left[Q M M^{\prime}\right]_{\left\{i^{\prime}\right\}} .
$$

Basta quindi sostituire nella (5), e ricordare la (4), per ottenere la $\left(10_{25}\right)$.

28. Avendo dianzi (nei nn. 26, 27) dimostrato i risultati enunciati nel n. 25 nell'ipotesi che si abbia $s=2$, non ci resta che da stabilirli per $s \geq 3$. All' uopo procederemo per induzione rispetto ad $s$, ammettendo quei risultati (oltre che per $s=2$ ) nel caso che in essi si sostituisca $s$ con $s-1$; e basterà provare le $\left(8_{95}\right),\left(9_{25}\right)$, poichè già sappiamo (n. 27) che la $\left(10_{25}\right)$ è conseguenza della $\left(8_{25}\right)$.

Consideriamo in $V$ le $s-1$ ipersuperficie

$$
A^{\prime}=A^{1}+A^{2}, \quad A^{3}, A^{4}, \ldots, A^{*},
$$


aventi ancora per somma $C$, e denotiamo con $V_{j}^{\prime}(j=1,2, \ldots, s-1)$ la loro funzione simmetrica elementare di grado $j$ e con $V_{f}^{*}(j=1,2, \ldots, s-2)$ l'analoga funzione simmetrica delle

$$
A^{3}, A^{4}, \ldots, A^{s} \text {. }
$$

Allora, posto $V_{0}^{*}=V, V_{s-1}^{*}=0$, risulta manifestamente

$$
V^{\prime}{ }_{j}=A^{\prime} V_{j-1}^{*}+V_{j}^{*} \quad(j=1,2, \ldots, s-1) .
$$

In virtù dell'ammessa induzione, possiamo applicare la $\left(8_{25}\right)$ alle $s-1$ ipərsuperficie (1), il che fornisce

$$
\bar{C}_{i}=\left[f^{\prime}\left(A^{\prime}\right)\right]_{\{i\}} \quad(i=0,1,2, \ldots),
$$

dove $f^{\prime}\left(A^{\prime}\right)$ sta per indicare la serie formale di potenze delle (1) definita dalla

$$
f^{\prime}\left(A^{\prime}\right)=\frac{V_{1}^{\prime}+2 V_{2}^{\prime}+\ldots+(s-1) V_{s-1}^{\prime}}{1-V_{2}^{\prime}-2 V_{3}^{\prime}-\ldots-(s-2) V_{s-1}^{\prime}} .
$$

Più precisamente, posto

$$
f^{\prime}\left(A^{\prime}\right)=\Sigma c^{\prime} Q A^{\prime[r]},
$$

dove $c^{\prime}, r$ sono interi non negativi e $Q$ designa un prodotto di potenze delle sole ipersuperficie (2), il cui grado denotiamo con $h$, la (4) non è che un modo compatto di scrivere la:

$$
\overline{C_{i}}=\Sigma c^{\prime}\left[Q A^{\prime}[r]\right]_{\{i\}},
$$

ossia, in forza della $\left(4_{25}\right)$, la :

$$
\overline{C_{i}}=\Sigma c^{\prime}\left(\overline{\left.Q A^{\prime[r]}\right)_{i-h-r+1}} .\right.
$$

D'altro canto, avuto riguardo alla prima delle (1), ed applicando la $\left(10_{25}\right)$ con $s=2$, vediamo che è

$$
\left(\overline{Q A^{\prime[r]}}\right)_{i-h-r+1}=\left[Q g(A)^{[r]}\right]_{\{i\}},
$$

dove $g(A)$ sta per indicare la serie di potenze di $A, A^{2}$ definita da

$$
g(A)=\frac{A^{1}+A^{2}+2 A^{1} A^{2}}{1-A^{1} A^{2}} .
$$

Pertanto la (7), tenuto conto della (6), fornisce

$$
\overline{C_{i}}=\Sigma c^{\prime}\left[Q g(A)^{[r]}\right]_{\mid i\}}=\left[\Sigma c^{\prime} Q g(A)^{[r]}\right]_{\{i\}}=\left[f^{\prime}(g(A))\right]_{\{i \mid},
$$

ossia vale la (8), dove $f(A)$ è la serie formale di potenze delle $A$ definita dalla

$$
f(A)=f^{\prime}(g(A)) \text {. }
$$


Tutto sta ora nel verificare che quest'ultima coincide con quella data dalla $\left(6_{25}\right)$; ciò si effettua con facili calcoli algebrici, poggiando sulle (5), (8) ed utilizzando, oltre alle (3), le identità :

$$
V_{j}=V_{j}^{*}+\left(A^{1}+A^{2}\right) V_{j-1}^{*}+A^{1} A^{2} V_{j-2}^{*},
$$

valide ovviamente per $j=1,2, \ldots, s$ fore si assuma $V_{-1}^{*}=V_{s}^{*}=0$ ).

Stabilita cosi in tutta generalità la $\left(8_{25}\right)$, e quindi pure la $\left(10_{25}\right)$, non ei resta che da dimostrare la $\left(9_{25}\right)$ (per $s>2$ ). A tal uopo, consideriamo i polinomi nelle $A$ :

$$
\varphi(A)=V_{1}+2 V_{2}+3 V_{3}+\ldots+s V_{s}, \quad \psi(A)=V_{2}+2 V_{3}+\ldots+(s-1) V_{s}
$$

(fra i quali passa la relazione $\varphi(A)-\psi(A)=V_{1}+V_{2}+\ldots+V_{s}$ ), e seriviamo più esplicitamente il secondo di essi sotto la forma

$$
\Psi(A)=\Sigma d Q
$$

dove $d$ è un intero e $Q$ denota un prodotto di potenze delle $A$, il cui grado indichiamo con $h$. Allora la $\left(6_{25}\right)$ fornisce

$$
f(A)=\frac{\varphi(A)}{1-\psi(A)}
$$

sicchè risulta identicamente :

$$
f(A)=\varphi(A)+\psi(A) f(A) .
$$

Pertanto dalla $\left(8_{25}\right)$, avuto riguardo alla $(10)$ ed alle convenzioni del n. 25, deduciamo

$$
\overline{C_{i}}=[\varphi(A)]_{\{i\}}+[\psi(A) f(A)]_{\{i\}}=[\varphi(A)]_{\{i\}}+\Sigma d[Q f(A)]_{\{i\}} .
$$

Da qui, poggiando sulle $\left(10_{25}\right),\left(4_{25}\right)$ ed applicando ancora una volta la $(10)$, traiamo:

Risulta dunque:

$$
\begin{aligned}
\overline{C_{i}}-[\varphi(A)]_{\{i\}} & =\Sigma d(\overline{Q C})_{i-h}=\Sigma d[Q C]_{\{i\}}=[(\Sigma d Q) C]_{\{i\}}= \\
& =[C \psi(A)]_{\{i\}} .
\end{aligned}
$$

$$
\overline{C_{t}}=[\varphi(A)+C \psi(A)]_{\{i\}}
$$

e quest' equazione, tenuto conto delle $(9),\left(4_{25}\right)$, non è che un modo compatto di scrivere la $\left(9_{25}\right)$.

29. In relazione ai risultati precedentemente ottenuti, faremo ora un' ulteriore estensione ed una verifica.

Consideriamo anzitutto su $V$ un qualunque numero $t(\geq 1)$ di serie d'ipersuperficie

$$
A^{l 1}, A^{l 2}, \ldots, A^{l s_{l}} \quad(l=1,2, \ldots, t),
$$


e poniamo

$$
\begin{gathered}
V_{i}^{l}= \pm A^{l l} A^{l 2} \ldots A^{l i} \\
C^{l}=A^{l 1}+A^{l 2}+\ldots+A^{l_{l}}, \\
f^{l}(A)=\frac{V_{1}^{l}+2 V_{2}^{l}+\ldots+s_{l} V_{s_{l}}^{l_{l}}}{1-V_{2}^{l}-2 V_{3}^{l}-\ldots-\left(s_{l}-1\right) V_{s_{l}}^{l}} .
\end{gathered} \quad\left(i=1,2, \ldots, s_{l}\right),
$$

Allora, se $Q$ d̀ un qualunque monomio $\left(4_{26}\right)$ di grado $h$ ed $r_{1}, r_{2}, \ldots, r_{t}$ denotano interi non negativi arbitrari, risulta:

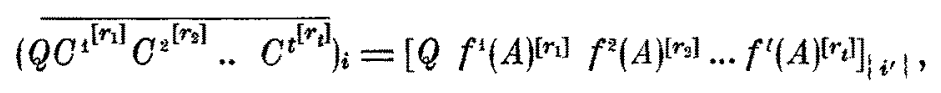

dove, per abbreviare, si è posto

$$
i^{\prime}=i+h+r_{1}+r_{2}+\ldots+r_{t}-1 .
$$

Infatti la (1) si riduce alla $\left(10_{25}\right)$ per $t=1 ; \theta$, per $t>1$, la si dimostra subito induttivamente poggiando sulla $\left(10_{25}\right)$ medesima.

Vediamo, in secondo luogo, come si possa verificare direttamente la $\left(8_{25}\right)$ nell' ipotesi semplificatrice che si abbia

$$
A^{*}=A^{2}=\ldots=A^{s}=A,
$$

e quindi

$$
C=s A \text {. }
$$

La $\left(1_{2 s}\right)$ mostra intanto che, se $P=A^{[s]}$, risulta

$$
\overline{P_{i}}=\left(\overline{\left.A^{[s]}\right)_{i}}=\left(\begin{array}{c}
s+i-1 \\
i
\end{array}\right) A^{[s+i]}\right.
$$

In particolare, e d'accordo col n. 14, si ha quindi $\bar{A}_{i}=A^{[i+1]}$, eppertanto:

$$
\bar{C}_{i}=(s A)^{[i+1]}=s^{i+1} A^{[i+1]} \text {. }
$$

Inoltre la $\left(4_{z 5}\right)$ fornisce

$$
\left(A^{[k]}\right]_{\{i\}}=\left(\overline{\left.A^{[k]}\right]_{i-k+1}}=\left(\begin{array}{c}
i \\
k-1
\end{array}\right)^{[[i+1]}\right.
$$

Osserviamo poscia che la $\left(5_{25}\right)$ attualmente fornisce $V_{i}=\left(\begin{array}{l}s \\ i\end{array}\right) A^{[i]}$, sicch̀̀ la $\left(6_{25}\right)$ diventa :

$$
f(A)=\frac{s A+2\left(\begin{array}{l}
s \\
2
\end{array}\right) A^{[2]}+3\left(\begin{array}{l}
s \\
3
\end{array}\right) A^{[3]}+\ldots+s A^{[s]}}{1-\left(\begin{array}{l}
s \\
2
\end{array}\right) A^{[2]}-2\left(\begin{array}{l}
s \\
3
\end{array}\right) A^{[3]}-\ldots-(s-1) A^{[s]}}
$$


Ora, argomentando ad esempio per induzione rispetto ad $i$, si stabiliscono agevolmente le identità

$$
i\left(\begin{array}{l}
s \\
i
\end{array}\right)=s(s-1)^{i-1}-s \sum_{j=0}^{i-3}(i-j-2)\left(\begin{array}{c}
s \\
i-j-1
\end{array}\right)(s-1)^{j} \quad(1 \leq i \leq s),
$$

dove la somma a secondo membro va soppressa per $i=1,2$; in virtù di queste, l'ultima relazione si riduce alla

$$
f(A)=s A+s(s-1) A^{[2]}+s(s-1)^{2} A^{[3]}+s(s-1)^{s} A^{[4]}+\ldots .
$$

Da qui si trae che, per $i=0,1,2, \ldots$, risulta:

$$
\begin{aligned}
{[f(A)]_{\{i\}} } & =s[A]_{\{i\}}+s(s-1)\left[A^{[2]}\right]\{i\} \\
& =s\left[1+s(s-1)^{2}\left[A^{[8]}\right]_{\{i\}}+\ldots=\right. \\
& =s[1+(s-1)]^{i} A^{[i+1]}=s^{i+1} A^{[i+1]} .
\end{aligned}
$$

Basta quindi confrontare con la (2), per dedurne la $\left(8_{95}\right)$.

30. Ci proponiamo da ultimo di dimostrare che:

La successione covariante di un'ipersuperficie $B$, che sia differenza di due date ipersuperficie $C, A$ di $V$, si calcola con la formula

$$
\bar{B}_{i}=[g(A, C)]_{|i|},
$$

nella quale $g(A, C)$ denota la serie formale di potenze di $A, C$ definita dalla funzione razionale

$$
g(A, C)=\frac{C-A}{1+2 A+A C} .
$$

Poichè $C=A \dashv B$, risulta $\bar{C}_{i}=[f(A, B)]_{\{i\}}$ (n. 25), dove

$$
f(A, B)=\frac{A+B+2 A B}{1-A B} .
$$

Scritta la (2) come serie di potenze:

$$
g(A, C)=\mathbf{s} c A^{[h]} C^{[r]},
$$

avuto riguardo alle $\left(4_{25}\right),\left(10_{25}\right)$ risulta

$$
\begin{gathered}
{\left[g(A, C]_{\{i\}}=\Sigma c\left[A^{[h]} C^{[r r}\right]_{\{i\}}=\Sigma c\left(\overline{A^{[h]} C^{[r]}}\right)_{i-h-r+1}=\right.} \\
\quad=\Sigma c\left[A^{[h]} f(A, B)^{[r]}\right]_{|i|}=\left[g(A, f(A, B)]_{\{i\}} .\right.
\end{gathered}
$$

Ne consegue la (1), osservando che le (2), (3) implicano la $g(A, f(A, B))=B$ e che d'altro canto, ancora per la $\left(4_{25}\right)$, si ha $B_{\{i\}}=\bar{B}_{i}$. 
Passiamo a dare forma esplicita all'equivalenza (1) dianzi stabilita. Anzitutto, avuto riguardo alla (2), con facile calcolo si vede che nel secondo membro della (4) la somma va estesa a tutte le coppie di interi $h, r$ non negativi soddisfacenti alla

$$
r \leq h+1
$$

esclusa la coppia $h=0, r=0$, e che il coefficiente $c$ vale

$$
c=(-1)^{h 2^{h-r-4}}\left[\left(\begin{array}{c}
h-1 \\
h-r-1
\end{array}\right)+4\left(\begin{array}{c}
h \\
h-r+1
\end{array}\right)\right],
$$

dove un simbolo combinatorio $\left(\begin{array}{l}a \\ b\end{array}\right)$ va posto uguale allo zero se $a<0$, o se $b<0$, o se $b>a$. Con tali precisazioni circa la serie di potenze (4), e posto per abbreviare:

$$
j=i-h-r+1,
$$

dalla (1) - avuto riguardo ai $\mathrm{nn} .25,17$ - si ricava

$$
\begin{gathered}
\bar{B}_{i}=\sum_{r \leq h+1} c\left(\overline{\left.A^{[h]} C^{[r}\right]}\right)_{j}= \\
=\sum_{r \leq h+1} c A^{[h]} C^{[r]} \sum_{l=0}^{j}\left(\begin{array}{c}
h+l-1 \\
l
\end{array}\right)\left(\begin{array}{c}
i-h-l \\
j-l
\end{array}\right) A^{[l]} C^{[j-l]} .
\end{gathered}
$$

L'espressione di $\bar{B}_{i}$ cosi ottenuta, risulta una forma di grado $i+1$, a coefficienti numerici, nelle $A, C$. Essa potrebbe venir raffrontata con quella che si desume dalla $B=C-A$ in base al $n$. 14, e cioè con la:

$$
\bar{B}_{i}=\sum_{l=0}^{i+1}(-1)^{l}\left(\begin{array}{c}
i+1 \\
l
\end{array}\right) A^{[l]} C^{[i-l+1]}
$$

deducendone delle identità che, per brevità, omettiamo di scrivere esplicitamente.

CAPITOLO TERZO

\section{ALCUNI PROBLEMI D'INTERSEZIONE}

\section{§ I. - Equivalenza funzionale di una varietà nell'intersezione di due o pì̀ altre.}

31. Prese in $V$ tre varieta $M, N, P$, per le quali conserviamo le notazioni e le ipotesi del primo capoverso del n. 9, ei proponiamo di determinare l' equivalenza funzionale $E$ di $P$ nell'intersezione $d i M$ ed $N$, ossia (n. 11) di esprimere la differenza

$$
E=(M N)_{V}-(M N)_{V}^{P}
$$

operando opportunamente - entro anelli di $V$ - sulle varietà covarianti delle $M, N, P$. 
A questo scopo, premettiamo alcune considerazioni sulla successione $\left|P^{0}\right|$, di sostegno $P$, definita ponendo:

$$
\left.\left.\left\{P^{\prime}\right\}=\left(\left(\mid P_{V}\right\} \mid \tilde{M}_{V}\right\}\right)_{M}\left\{\tilde{N}_{V}\right\}\right)_{N} \text {. }
$$

Si vede anzitutto ch'essa dipende in modo simmetrico da $M$ ed $N$, risultando

$$
\left.\left|P^{0}\right|=\left(|| P_{V}\left|\quad \tilde{N}_{V}\right|\right)_{N}\left|M_{V}\right|\right)_{M}
$$

in base al n. 5, f). Inoltre, applicando alle $P \subset M \subset V, P \subset N \subset V$ il teorema dell' appartenenza nella forma $\left(4_{18}\right)$, le (2), (3) forniscono:

$$
\left\{P^{0}\right\}=\left(\left|P_{M}\right|\left|\tilde{N}_{V}\right|\right)_{N}=\left(\left|P_{N}\right|\left|\tilde{M}_{V}\right|\right)_{M} \text {. }
$$

La dipendenza simmetrica di $\left\{P^{0}\right\}$ da $M$ ed $N$ risulta anche direttamente dalla formula

(5)

che ora dimostreremo.

$$
\left.\left|P^{0}\right|=\left(\left|P_{M}\right|\left|P_{N}\right| \mid \tilde{P}_{V}\right\}\right)_{P}
$$

A tal fine notiamo che, in virtù della $\left(7_{8}\right)$, risulta

$$
\left(\left(\left\{P_{V}\left|\left\{\tilde{M}_{V} \mid\right)_{M}\right| \tilde{P}_{V}\right\}\right)_{P}=\left(\left(\mid \tilde{P}_{V}\right\}\left|P_{V}\right|\right)_{P}\left\{\tilde{M}_{V}\right\}_{M}=\left(P\left|\tilde{M}_{V}\right|\right)_{M}\right. \text {. }
$$

Pertanto, avuto riguardo alla $\left(4_{18}\right)$ ed all'ultima proposizione del $\mathrm{n}$. 8 , si ottiene: $\left.\left.\left.\left.\left(\left\{P_{M}|| P_{N} \mid\left\{\tilde{P}_{V}\right\}\right)_{P}=\left(|| P_{V}\right\} \mid \tilde{M}_{V}\right\}\right)_{M} \mid P_{N}\right\}\left\{\tilde{P}_{V} \mid\right)_{P}=\left(\left\{P\left|\tilde{M}_{V}\right|\right)_{M}\right\} P_{N}\right\}\right)_{P}=$ $\left.=\left(\mid P_{N}\right\}\left|\tilde{M}_{V}\right|\right)_{M}$

e da qui, in base alla (4), segue la (5).

Osserviamo poi che, in virtù delle $\left(1_{9}\right),\left(3_{9}\right)$, l'intero

$$
t=p-q=p+v-m-n
$$

risulta non negativo. Si risponde allora alla questione postaci in principio col seguente

Teonema. - L'equivalenza funzionale (1) di $P$ nell' intersezione di $M$ ed $N$ in $V$ si esprime con la

$$
E=P^{0}{ }_{t},
$$

la successione $\left\{P^{0} \mid\right.$ essendo quella dianzi definita e $t$ essendo dato dalla (6) (12).

Avuto riguardo alle (1)-(5), la (7) può scriversi in modo più esplicito sotto una qualunque delle forme:

$$
\begin{aligned}
(M N)_{V}-(M N)_{V}^{P} & =\sum_{i=0}^{t} \sum_{j=0}^{i}\left(\left(P_{V, j} \tilde{M}_{V, i-j}\right)_{M} \tilde{N}_{V, t-i)_{N}=}=\right. \\
& =\sum_{i=0}^{t}\left(P_{M, i} \tilde{N}_{V, t-i}\right)_{N}= \\
& =\sum_{i=0}^{t} \sum_{j=0}^{t-i}\left(P_{M, i} P_{N, j} \tilde{P}_{V, t-i-j}\right)_{P}
\end{aligned}
$$

(12) In base a risultati che verranno stabiliti nel Cap. $7^{\circ}$, si potrebbe dimostrare che la formula fornita dal precedente teorema quando in particolare vi si faccia $p=m-1$ (e quindi $t=v-n-1$ ) coincide con la (7.02) in ToDd [8]. 
e sotto quelle che si deducono dalle prime due con lo scambio di $M$ ed $N$. Rileviamo che, tenuto conto dei nn. 6, 9 e della (6), ciascuno dei membri delle $(7),(8)$ ha la dimensione $p-t=q$, e si riduce a $P$ per $t=0$. Ciò intanto dimostra il suddetto teorema nell' ipotesi che sia $t=0$.

Osservato che gli interi $m$ ed $n$ sono inferiori a $v$ e che nessuno di essi può risultare inferiore a $p$, procederemo alla dimostrazione di quel teorema distinguendo i quattro casi seguenti :

$$
\begin{array}{ll}
1^{\circ}: p=m ; & 2^{\circ}: n=v-1, \quad p<m ; \\
3^{\circ}: n<v-1, \quad p=m-1 ; & 4^{\circ}: n<v-1, \quad p<m-1 .
\end{array}
$$

32. Nel primo dei casi dianzi indicati si ha $P=M$; ciò implica (n. 9) che la varietà

$$
Q=(M N)_{V}^{P}
$$

si riduca allo zero e che (n. 18) la successione $\left|P_{M}\right|=\left\{M_{M} \mid\right.$ non sia altro che la $M, 0,0, \ldots$. Poichè la $\left(6_{31}\right)$ ora fornisce $t=v-n$, cosi la relazione $\left(8_{31}\right)$ da stabilire diventa:

$$
(M N)_{V}=\left(M \tilde{N}_{V, v-n}\right)_{N}
$$

In virtù del n. 23, l'ultima relazione equivale alla

$$
(M N)_{V}=\left(M\left(N^{[2]}\right)_{V}\right)_{N}
$$

e questa è certamente soddisfatta, in forza delle $M=P \subset N$ (n. 5 , a").

Nel secondo dei quattro casi contemplati alla fine del n. $31, N$ è una ipersuperficie di $V$ non contenente $M$. Pertanto le due componenti $P$ e $Q$ dell'interferenza $\langle M N\rangle$ di $M$ e $N$ hanno entrambe la dimensione $m-1$. Si ha quindi $p=q$, ossia $t=0$, ed il teorema da dimostrare sussiste in base al penultimo capoverso del n. 31 .

33. Nel terzo caso, procederemo per induzione rispetto al carattere $v-n$ relativo al prodotto simbolico $(M N)_{V}^{P}$ (il quale risulta ora $>1$ ). Poichè, in base ai casi $1^{\circ}$ e $2^{\circ}$ trattati nel numero precedente, il teorema del $n .31$ vale per il valore 1 di tale carattere, così potremo ammettere che il teorema stesso sussista per prodotti simbolici aventi il carattere $v-n-1$. Prendiamo in $V$ un'ipersuperficie $V^{\prime}$ passante genericamente per $N$ : questa taglierà la $M$ lungo $P$ e, ulteriormente, lungo una varietà $M^{\prime}$ di dimensione $m^{\prime}=m-1=p$. È chiaro che quest'ultima passerà per $Q$, e segherà $N$ (semplicemente) lungo $Q$ e, ulteriormente, lungo una varietà $P^{\prime}$ appartenente a $P$, luogo dei punti comuni ad $M^{\prime}, P$. Poichè $M^{\prime}$ e $P$ sono due ipersuperficie di $M$ prive di componenti comuni, cosi $P^{\prime}$ avrà la dimensione $p^{\prime}=m-2=p-1$ e risulterà

$$
\begin{aligned}
P^{\prime} \doteq\left(M^{\prime} P\right) & =\left(M^{\prime} P\right)_{M}, \\
P+M^{\prime} \doteq\left(M V^{\prime}\right) & =\left(M V^{\prime}\right)_{V} .
\end{aligned}
$$


Inoltre $Q$, non soltanto è la componente regolare dell'intersezione di $M, N$ in $V$ residua a $P$, ma è anche l'intersezione regolare di $M^{\prime}, N$ in $V^{\prime}$ residua a $P^{\prime}$, sicchè :

$$
(M N)_{V}^{P}=\left(M^{\prime} N\right)_{V}^{P^{\prime}}
$$

Poichè per quest'ultimo prodotto simbolico il suddetto carattere vale $v^{\prime}-n=v-n-1$, mentre la $\left(6_{31}\right)$ ora fornisce:

$$
t^{\prime}=p^{\prime}+v^{\prime}-m^{\prime}-n=t-1,
$$

così per l'ammessa induzione avremo:

$$
\left(M^{\prime} N\right)_{V^{\prime}}-\left(M^{\prime} N\right)_{V^{\prime}}^{P^{\prime}}=\sum_{i=0}^{t-1}\left(P_{M^{\prime}, i}^{\prime} \tilde{N}_{V^{\prime}, t-i-1}\right)_{N}
$$

In virtù della (1) e del teorema della sezione (n. 19), si ha inoltre

$$
P_{M r, i}^{\prime}=\left(P_{M, i} M^{\prime}\right)_{M} \text {. }
$$

Da qui, tenuto conto della (2) e del n. 5, a), discende

$$
\begin{aligned}
P_{M^{\prime}, i}^{\prime} & =\left(P_{M, i}\left(M V^{\prime}\right)_{V}\right)_{M}-\left(P_{M, i} P\right)_{M L}= \\
& =\left(P_{M, i} V^{\prime}\right)_{V}-\left(P_{M, i} P\right)_{M} ;
\end{aligned}
$$

e poichè (n. 14): $P_{M, i}=(-1)^{i}\left(P^{[i+1]}\right)_{M}$, ne consegue

$$
P_{M M^{\prime}, i}^{\prime}=\left(P_{M, i} V^{\prime}\right)_{V}+P_{M, i+1} \text {. }
$$

Se si applica alla terna $N \subset V^{\prime} \subset V$ il teorema dell'appartenenza nella forma $\left(5_{18}\right)$, si ottiene

$$
\left\{\tilde{N}_{V}\right\}=\left(\left\{\tilde{N}_{V^{\prime}}\right\}\left\{\tilde{V}_{V}^{\prime}\right\}\right)_{V^{\prime}} \text {. }
$$

Avuto riguardo al n. 14 ed al n. 5, a), si ha dunque:

$$
\tilde{N}_{V, i}=\tilde{N}_{V^{\prime}, i}+\left(\tilde{N}_{V^{\prime}, i-1} V^{\prime}\right)_{V}
$$

Osservato che, in forza delle $\left(6_{31}\right), p=m-1$, attualmente risulta :

$$
v^{\prime}-n=t
$$

basta applicare successivamente il n. 5, a), la (2), ed i nn. 5 a"), 23, per dedurre la catena di uguaglianze

$$
\begin{aligned}
(M N)_{V} & =\left(\left(M V^{\prime}\right)_{V} N\right)_{V^{\prime}}=(P N)_{V^{\prime}}+\left(M^{\prime} N\right)_{V^{\prime}}= \\
& =\left(P\left(N^{[2]}\right)_{V^{\prime}}\right)_{N}+\left(M^{\prime} N\right)_{V^{\prime}}=\left(P \tilde{N}_{V^{\prime}, t}\right)_{N}+\left(M^{\prime} N\right)_{V^{\prime}} .
\end{aligned}
$$

Sommando a membro a membro la (4) con la relazione che da qui si ottiene uguagliando il primo e l'ultimo membro, e quindi applicando le (3), (5) ed il n. 5, c), si ricava:

$$
\begin{aligned}
(M N)_{V}-(M N)_{V}^{P} & =\left(P \tilde{N}_{V^{\prime}, t}\right)_{N}+\sum_{i=0}^{t-1}\left(P_{M^{\prime}, i}^{\prime} \tilde{N}_{V^{\prime}, t-i-1}\right)_{N}= \\
& =\left(P \tilde{N}_{V^{\prime}, t}\right)_{N}+\sum_{i=0}^{t-1}\left(P_{M, i}\left(\tilde{N}_{V^{\prime}, t-i-1} V^{\prime}\right)_{V}\right)_{N}+\sum_{i=0}^{t-1}\left(P_{M, i+1} \tilde{N}_{V^{\prime}, t-i-1}\right)_{N}
\end{aligned}
$$


Nella prima dolle dne sommatorie scritte per ultime, è lecito far andare l'indice $i$ da 0 a $t$, essendo $\tilde{N}_{V,-1}=0$ (n. 15); inoltre, poichè $P=P_{M, 0}$, la somma dei rimanenti termini dell'ultima riga, ove si assuma $i+1$ come nuovo indice di sommazione, diventa

$$
\sum_{i=0}^{t}\left(P_{M, i} \tilde{N}_{V^{\prime}, t-i}\right)_{N}
$$

Facendo infine di quell'ultima riga un'unica somma rispetto ad $i$ variante da da 0 a $t$, ed applicando la (6), si ha subito la formula $\left(8_{3_{1}}\right)$ che si voleva stabilire.

34. Nell' ultimo dei quattro casi considerati alla fine del $\mathrm{n}$. 31 , procederemo per induzione rispetto al carattere $m-p$ del prodotto simbolico $(M N)_{V}^{P}$ (il quale, attualmente, risulta $>1$ ). Scelta in $V$, come dianzi, un'ipersuperficie $V^{\prime}$ passante per $N$, denotiamo con $M^{\prime}$ la varietà pura di dimensione $m^{\prime}=m-1$, intersezione di $M$ e $V^{\prime}$. Poichè dalle nostre ipotesi risulta $m^{\prime}>p$, così ora $M^{\prime}$, pur passando (semplicemente) per $P$, non contiene più la $P$ quale componente. Pertanto, accanto alla

$$
M^{\prime} \doteq\left\langle M V^{\prime}\right\rangle=\left(M V^{\prime}\right)_{V}
$$

attualmente si ha che è $\left\langle M^{\prime} N\right\rangle \doteq P+Q$, e quindi (n. 9):

$$
(M N)_{V^{r}}^{P}=\left(M^{\prime} N\right)_{V^{\prime}}^{P}
$$

Dalla (1) discende inoltre (n. 5, a)) la :

$$
(M N)_{V}=\left(M^{\prime} N\right)_{V^{\prime}}
$$

Siccome il carattere suddetto nel caso del prodotto simbolico $\left(M^{\prime} N\right)_{V}^{P}$, vale

$$
m^{\prime}-p=(m-p)-1,
$$

così - per l'ammessa induzione - è lecito applicare il teorema del n. 31 all'equivalenza funzionale di $P$ nell' intersezione di $M^{\prime}$ ed $N$ in $V^{\prime}$. Per tale intersezione, il carattere analogo a $\left(6_{31}\right)$ è dato da

e si ha quindi

$$
t^{\prime}=p+v^{\prime}-m^{\prime}-n=t
$$

$\left(M^{\prime} N\right)_{V^{\prime}}-\left(M^{\prime} N\right)_{V^{\prime}}^{P}=\sum_{i=0}^{t}\left(P_{N, i} \tilde{M}_{\left.V^{\prime}, t-i\right) \bar{M}^{\prime}}^{\prime}\right.$

Infine, se si rammenta il teorema della sezione nella forma $\left(4_{12}\right)$, si vede che è

$$
\tilde{M}_{V^{\prime}, t-i}^{\prime}=\left(\tilde{M}_{V, t-i} V^{\prime}\right)_{V}
$$

Sostituendo nella (4), ed usufruendo delle (1), (2), (3) e del n. 5, d'), si ottiene senz'altro il risultato che ci eravamo proposti di stabilire (n. 31), sotto la forma:

$$
(M N)_{V}-(M N)_{V}^{P}=\sum_{i=0}^{t}\left(P_{N, i} \tilde{M}_{V, t-i}\right)_{M}
$$


35. Generalizzeremo ora il risultato del n. 31 riferendoci ad $s(\geq 2)$ varieta $M^{1}, M^{2}, \ldots, M^{s}$ di $V$, intersecantisi (regolarmente o no) lungo una varieta $P$, per le quali adottiamo le notazioni e le ipotesi del n. 9. A tal uopo, consideriamo la successione $\left\{P^{0}\right\}$ - di sostegno $P$ - definita dalla:

$$
\left|P^{0}\right|=\left(\ldots\left[\left(\left|P_{V}\right|\left|\tilde{M}_{V}^{1}\right|\right)_{M^{1}}\left|\tilde{M}_{V}^{2}\right|\right]_{M^{a}} \ldots\left|\tilde{M}_{V}^{s}\right|\right)_{M s} .
$$

Mediante considerazioni analoghe a quelle svolte nel n. 31 per la $\left(2_{3 !}\right)$, si constata l'indipendenza di questa successione dall'ordine in cui vi figurano le $M$, e si perviene di fatto a scrivere la (1) sotto la seguente forma, simmetrica rispetto alle $M$ :

$$
\left|P^{0}\right|=\left(\left|P_{M^{1}}\right| \cdot\left|P_{M^{2}}\right| \ldots\left|P_{M S}\right| \cdot\left|\tilde{P}_{V}\right|^{[s-1]}\right)_{P}\left({ }^{13}\right) .
$$

Designamo inoltre con $t$ l'intero non negativo

$$
t=p-q=p+(s-1) v-\left(m^{1}+m^{2}+\ldots+m^{s}\right) .
$$

Possiamo allora enunciare il

Teorema. - L'equivalenza funzionale $E$ di $P$ nell'intersezione delle $M^{i}$, $M^{2}, \ldots, M^{s}$ in $V$ si esprime con la

$$
E=\left(M^{2} M^{2} \ldots M^{s}\right)_{V}-Q=P_{t}^{0},
$$

dove $\left|P^{0}\right|$ e $t$ son date dalle (1)-(3), il che val quanto dire che risulta:

$$
\begin{aligned}
& \left(M^{1} M^{2} \ldots M^{s}\right)_{V}-\left(M^{1} M^{2} \ldots M^{s}\right)_{V}^{P}= \\
& (5)=\sum_{i_{1}=0}^{t} \sum_{i_{2}=i_{1}}^{t} \sum_{i_{3}=i_{2}}^{t} \ldots \sum_{i_{3}=i_{s-1}}^{t}\left(\ldots\left[\left(P_{V, i_{1}} \tilde{M}_{V, i_{2}-i_{1}}^{1}\right)_{M^{1}} \tilde{M}_{V, i_{3}-i_{3}}^{2}\right]_{M^{2}} \ldots \tilde{M}_{V, t-i_{s}}^{s}\right)_{M *}=
\end{aligned}
$$

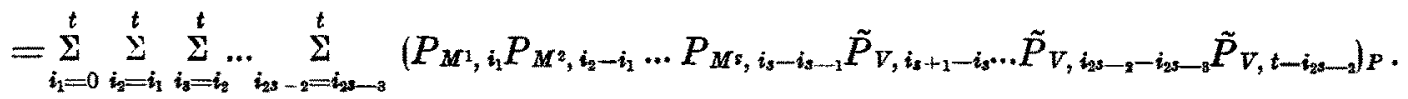

Questo teorema si riduce a quello del n. 31 per $s=2$. Potremo quindi supporre $s \geq 3$, ed ammettere la validita del teorema per l'intersezione di $s-1$ varietà. Scriviamo per comodità :

$$
\begin{array}{ll}
M^{s}=V^{\prime}, & M^{\prime j}=\left(M^{j} V^{\prime}\right)_{V} \\
m^{s}=v^{\prime}, & m^{\prime j}=m^{j}+v^{\prime}-v
\end{array} \quad(j=1,2, \ldots, s-1),
$$

ed osserviamo che, in virtù del n. $\left.5, a^{\prime}\right)$ e del n. 10, b), risulta :

$$
\left(M^{1} M^{2} \ldots M^{s}\right)_{V}=\left(M^{\prime 1} M^{\prime 2} \ldots M^{\prime s-1}\right)_{V}, \quad\left(M^{1} M^{2} \ldots M^{s}\right)_{V}^{P}=\left(M^{\prime 1} M^{\prime 2} \ldots M^{\prime s-1}\right)_{V}^{P} .
$$

Da queste relazioni, sottraendole a membro a membro, si deduce che l'equivalenza funzionale $E$ cercata uguaglia quella di $P$ nell'intersezione delle

(13) L'equivalenza fra la (1) e la (2) verrà altrimenti controllata nel n. 74. 
B. Segre: Nuovi motodi e risultati nella geometria ecc.

$M^{\prime 1}, M^{\prime 2}, \ldots, M^{\prime s-1}$ in $V^{\prime}$. A norma dell' ammessa induzione si ha quindi

$$
E=P_{t^{\prime}}^{\prime 0}
$$

dove si definisca la successione $\left\{P^{\prime 0}\right\}$ (di sostegno $P$ ) mediante la

$$
\left.\mid P^{\prime 0}\right\}=\left(\ldots\left[\left(\mid P_{V^{\prime}}\right\}\left\{\tilde{M}_{V^{\prime}}^{1} \mid\right)_{M^{n}}\left\{\tilde{M}_{V^{\prime}}^{\prime 2}\right\}\right]_{M^{\prime 2}} \ldots\left|\tilde{M}_{V^{\prime}}^{s-1}\right|_{M^{\prime s-1}},\right.
$$

ed inoltre, avuto riguardo alle (3), (6), si assuma

$$
t^{\prime}=p+(s-2) v^{\prime}-\left(m^{\prime 1}+m^{\prime 2}+\ldots+m^{\prime s-1}\right)=t .
$$

D'altro canto, in virtù dei teoremi dell'appartenenza (n. 18) e della sezione (n. 19), risulta:

$$
\begin{aligned}
& \left.\left\{P_{V^{\prime}}\right\}=\left(\left\{P_{V}\right\}\left\{\tilde{V}_{V}^{\prime}\right\}\right)_{V^{\prime}}=\left(\left\{P_{V}\right\} \mid \tilde{M}_{V}^{s}\right\}\right)_{M} s \\
& \quad\left\{\tilde{M}_{V^{\prime}}^{\prime}\right\}=\left(\left\{\tilde{M}_{V}^{j}\right\} \cdot V^{\prime}\right)_{V}=\left(\left\{\tilde{M}_{V}^{j}\right\} \cdot M^{s}\right)_{V} \quad(j=1,2, \ldots, s-1) .
\end{aligned}
$$

Basta quindi sostituire nella (8), applicare ripetutamente il n. 5, d'), e ricordare la simmetria della (1) rispetto alle $M$, per dedurre che è :

$$
\left\{P^{\prime 0}\right\}=\left\{P^{0}\right\} \text {. }
$$

Da qui segue subito la (4), in base alle (7), (9).

\section{§ II. - Intersezioni residue e varietà d'appoggio.}

36. Se è nota una componente pura $P$ dell'intersezione di due varietà $M, N$ di $V$ (la quale, come tale, possa avere dimensione $p$ regolare od irregolare), e nell'ipotesi che $M$ ed $N$ passino in modo generico (e quindi semplicemente) per $P$, l'intersezione di $M$ ed $N$ consta di $P$ - contata semplicemente - e di una residua varietà

$$
Q \doteq(M N)-P
$$

di dimensione $q$ regolare, la cui classe d'equivalenza è stata designata (n. 9) con

$$
Q=(M N)_{V}^{P}
$$

Per determinare - in funzione delle sole $M, N, P$ e loro varietà covarianti tale interseaione residua $Q$, basta ricorrere al teorema dẻl $n$. 31, il che fornisce:

$$
Q=(M N)_{V}-P_{t}^{0},
$$

dove $t$ si esprime con la $\left(6_{31}\right)$ e $P_{t}^{0}$ è dato da una qualunque delle somme che compaiono nella $\left(8_{31}\right)$.

Più in generale, a norma dei nn. 9, 35, l'intersezione $Q$ di date varietă $M^{1}, M^{2}, \ldots, M^{s}$ di $V$ residua ad una varietà nota $P$ ad esse comune, nell'ipotesi che la dimensione $q$ di $Q$ sia regolare, mentre $P$ abbia dimensione $p(\geq q)$ arbitraria, vien definita funzionalmente - mediante le sole $M, P e$ 
loro varietà covarianti - dall'equivalenza

$$
Q=\left(M^{i} M^{2} \ldots M^{s}\right)_{V}-P_{t}^{0}
$$

ora, in questa, $t$ si esprime con la $\left(3_{35}\right)$, e $P_{t}^{0}$ è dato dal secondo o terzo membro della $\left(5_{35}\right)$.

37. In base al n. 12, le varietà $P$ e $Q$ dianzi considerate si appoggiano mutuamente lungo una varietà pura $R$, di dimensione $q-1$, questa varietà essendo generalmente non nulla se $q \geq 1$. Dimostreremo al riguardo il seguente

Teorema. - Se $q \geq 1$ (e quindi $p \geq t+1)$, la suddetta varietò d'appoggio $R$ si esprime in funzione delle $M, P$ mediante l' equivalenza

$$
R=P^{0}{ }_{t+1},
$$

dove le notazioni sono quelle richiamate nel $n .36$.

Riferendoci al caso dell'intersezione di un qualunque numero $s(\geq 2)$ di varietà $M^{1}, M^{2}, \ldots, M^{s}$ di $V$, e ciò̀ supposto

$$
\left\langle M^{1} M^{z} \ldots M^{s}\right\rangle \doteq P+Q
$$

in base alla fine del n. 12 risulta:

$$
R=\left(Q V^{\prime}\right)_{V}-\left(M^{1} M^{*} \ldots M^{s} V^{\prime}\right)_{V}^{P}
$$

dove $V^{\prime}$ denota un'ipersuperficie di $V$ passante per $P$, scelta ivi in modo arbitrario purchè generico.

D'altro canto, applicando due volte il risultato finale del n. 36 , abbiamo:

$$
\begin{aligned}
& \left(Q V^{\prime}\right)_{V}=\left(M^{\prime} M^{2} \ldots M^{s} V^{\prime}\right)_{V}- \\
& -\sum_{i_{1}=0}^{t} \sum_{i_{2}=i_{1}}^{t} \ldots \sum_{i_{s=i s-1}}^{t}\left(\left[\ldots\left(P_{V, i_{1}} \tilde{M}_{V, i_{2}-i_{1}}^{1}\right)_{M^{1}} \ldots \tilde{M}_{V, t-i_{s}}^{s}\right]_{M s} V^{\prime}\right)_{V}, \\
& \left(M^{1} M^{z} \ldots M^{s} V^{\prime}\right)_{V}^{P}=\left(M^{1} M^{z} \ldots M^{s} V^{\prime}\right)_{V}
\end{aligned}
$$

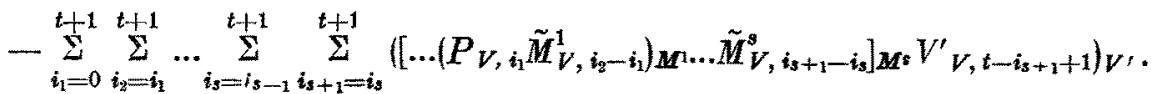

Ora è (n. 14):

$$
\left|\tilde{V}^{\prime}\right|=V^{\prime},\left(V^{[2]}\right)_{V}, 0,0, \ldots ;
$$

sicche i termini non nulli della sommatoria che figura nella (4) sono soltanto quelli obe provengono dai valori $t+1$ e $t$ dell'indice $i_{s+1}$. In forza della $\left(1_{35}\right)$, la somma dei primi vale precisamente $P_{t+1}^{0}$. Nel fare la somma dei secondi $\left(i_{s+1}=t\right)$, si possono omettere i termini che provengono dal valore $t+1$ dell'indice $i_{s}$, in quanto in essi compare il fattore

$$
\tilde{M}_{V, i_{s+1}-i_{s}}^{s}=\tilde{M}_{V,-1}^{s}=0 ;
$$


similmente, essendo per i termini che restano $i_{s} \leq t$, basta limitarci a quelli con $i_{s-1} \leq t$, in quanto nei rimanenti $\left(i_{s-1}=t+1\right)$ compare il fattore nullo $\tilde{M}_{i_{s}-i_{s-1}}^{s-1}$; e così via. La (4) equivale dunque alla:

$$
\begin{aligned}
\left(M^{1} M^{2} \ldots M^{s} V^{\prime}\right)_{V}^{P} & =\left(M^{1} M^{2} \ldots M^{s} V^{\prime}\right)_{V}-P_{t+1}^{0}- \\
& -\sum_{i_{1}=0}^{t} \sum_{i_{2}=i_{2}}^{t} \ldots \sum_{i_{s}=i_{s}-1}^{t}\left(\left[\ldots\left(P_{V, i_{1}} \tilde{M}_{V, i_{-}-i_{1}}^{t}\right) \ldots \tilde{M}_{V, t-i_{s}}^{s}\right]_{M^{s}}\left(V^{\prime}[2]\right)_{V}\right)_{V^{\prime}} ;
\end{aligned}
$$

e l'ultima sommatoria risulta uguale a quella ohe compare nella (3), in forza. del n. $\left.5, \mathbf{a}^{\prime \prime}\right)$.

Si ottiene quindi subito la formula (1) da stabilire, sottraendo a membro a membro le (3), (5) ed applicando la (2).

\section{§ III. - Successioni covarianti di alcune delle varietà dianzi considerate.}

38. Con le notazioni del paragrafo precedente, possiamo proporci di determinare le varietà covarianti delle $Q, R$ in funzione delle $M, P$ e loro varietà covarianti. La questione presenta notevoli difficoltà algoritmiche, e ci limiteremo a trattarla in qualche caso particolare.

Il caso più semplice si ha quando manchi la $P$, e quindi risulti

$$
Q=\left(M^{1} M^{2} \ldots M^{s}\right)_{V} .
$$

Allora manca la $R$, e le varietà covarianti $Q_{V, i}$ son date dalla $\left(7_{2 n}\right)$ (teorema del prodotto). Tenuto anche conto del n. 5, $\left.\mathrm{a}^{\prime \prime \prime}\right)$, si ha così che la (1) implica le:

(2) $\left.\left.\left\{Q_{V}\right\}=\left(\left\{M_{V}^{1}\right\} \cdot\left\{M_{V}^{2}\right\} \ldots\left\{M_{V}^{s}\right\}\right)_{V}=\left(\ldots\left[\left(Q \mid M_{V}^{1}\right\}\right)_{M^{1}} \cdot \mid M_{V}^{2}\right\}\right]_{M^{a}} \ldots\left\{M_{V}^{s}\right\}\right)_{M^{s}}$.

39. Passiamo ora ad esaminare il caso in cui $P$ abbia dimensione regolare, e ciò̀ risulti

$$
t=p-q=0,
$$

e sia inoltre $q \geq 1$, Allora $R$ è un' ipersuperficie tanto di $P$ che di $Q$, data da (n. 37):

$$
R=P_{1}^{0}=\sum_{i_{1}=0}^{1} \underset{i_{2}=i_{1}}{\stackrel{1}{\Sigma} \ldots} \underbrace{1}_{i_{s}=i_{s-1}}\left(\ldots\left[\left(P_{V, i_{1}} \tilde{M}_{V, i_{2}-i_{1}}^{1}\right)_{M^{1}} \tilde{M}_{V, i_{3}-i_{2}}^{2}\right]_{M^{2}} \ldots \tilde{M}_{V, 1-i_{s}}^{s}\right)_{M s} .
$$

Poichè nell'ultima somma gli indici $i_{1}, i_{2}-i_{1}, i_{3}-i_{2}, \ldots, 1-i_{s}$ sono numeri interi non negativi aventi per somma 1 , cos̀̀ quella somma conterrà comples. sivamente $s+1$ termini, corrispondenti a valori di quegli indici tutti nulli tranne uno uguale all' unita. Tenuto presente che $\dot{e}($ nn. 6, 7):

$$
P_{V, 0}=P, \quad \tilde{M}_{V, 0}^{l}=M^{l}, \quad \tilde{M}_{V, 1}^{l}=-M_{V, 1}^{l} \quad(l=1,2, \ldots, s),
$$


risulta pertanto

$$
R=P_{V, 1}-\sum_{l=1}^{1}\left(P M_{V, 1}^{l}\right)_{M^{l}}
$$

La (1) permette di esprimere in funzione delle $P, M, P_{1}, M_{1}$, e con sole operazioni negli anelli $\mathfrak{A}_{P}, \mathfrak{A}_{M}, \mathfrak{A}_{V}$, ciascuna delle $\left(R^{[i]}\right)_{P}$, e quindi pure ciascuna delle $R_{P, i}$ tenuto conto che è (n. 14):

$$
R_{P, i}=(-1)^{i}\left(R^{[i+1]}\right)_{P} \quad(i=0,1,2, \ldots) .
$$

Da qui si deducono subito le $R_{V, i}$, poichè (n. 18) dalle $R \subset P \subset V$ segue che ̀े $\left.\left\{R_{V}\right\}=\left(\mid R_{P}\right\}\left\{P_{V}\right\}\right)_{P}$, sicchè :

$$
R_{V, i}=\sum_{j=0}^{i}(-1)^{j}\left(\left(R^{[j+1]}\right]_{P} P_{V, i-j}\right)_{P} .
$$

Allo scopo di determinare le $Q_{V, i}$ in funzione delle $M, P$ e loro varietà covarianti, fissiamo un qualunque intero $k$ compreso fra 1 e $q$, e consideriamo in $V k$ ipersuperficie

$$
A^{1}, A^{2}, \ldots, A^{k}
$$

passanti genericamente per $Q$. La $A^{\prime}$ segherà $P$ lungo la $R$ e, ulteriormente, lungo una varietà $P^{\prime}$, di dimensione $p-1=r$, e si arrà :

$$
P^{\prime}=\left(P A^{i}\right)_{V}-R \quad(i=1,2, \ldots, k) .
$$

Nell' ipotesi che sia $k \leq v-q$, il che non è limitativo per $k(\leq q)$ se si suppone $v \geq 2 q$, le $A^{l}$ si segano lungo una varietà $M$, passante per $Q$, di dimensione regolare $v-k$. Risulta allora:

$$
\begin{gathered}
M=\left(A^{1} A^{2} \ldots A^{k}\right), \\
\left\langle M^{1} M^{2} \ldots M^{k} M\right\rangle \doteq Q+H,
\end{gathered}
$$

dove $H$ denota la varieta intersezione delle $M^{1}, \ldots, M^{s}, M$ residua a $Q$; questa ha dimensione regolare:

$$
m^{4}+m^{2}+\ldots+m^{\varepsilon}+(v-k)-s v=q-k,
$$

e soddisfa manifestamente (n. 9) all'equivalenza

$$
H=\left(M^{4} M^{2} \ldots M^{s} M\right)_{V}^{Q}
$$

ed inoltre alla :

$$
H=\left(P^{\prime \prime} P^{\prime 2} \ldots P^{\prime k}\right)_{P}
$$

In virtù del n. 35, dalle (5), (6) si trae che è :

$$
\begin{gathered}
\left(M^{1} M^{2} \ldots M^{s} M\right)_{V}=H+ \\
+\sum_{i_{1}=0}^{k} \sum_{i_{2}=i_{1}}^{k} \ldots \sum_{i_{s}=i_{s-1}}^{k} \sum_{i=i_{s}}^{k}\left[\left(\ldots\left[\left(Q_{V, i_{1}} \tilde{M}_{V, i_{2}-i_{1}}^{k}\right)_{M^{1}} \tilde{M}_{V, i_{2}-i_{2}}^{2}\right]_{M M^{2}} \ldots \tilde{M}_{V, i-i_{s}}^{s}\right)_{M_{s}} \tilde{M}_{V, k-i}\right]_{M} .
\end{gathered}
$$


Ora la (4) fornisce senz' altro (n. 17):

$$
\check{M}_{V, k-i}=\left(M \cdot V_{k-i}(A)_{V} \quad(i=0,1, \ldots, k),\right.
$$

dove $V_{k-i}(A)$ indica la funzione simmetrica elementare di grado $k-i$ delle ipersuperficie (2) di V. D'altro canto dalla (7), poggiando sulle (3) e sul n. 5, a), si deduce:

$$
H=\sum_{i=0}^{k}(-1)^{i}\left(\left(R^{[i]}\right]_{P} V_{k-i}(A)\right)_{V} .
$$

Se dunque sostituiamo in (8) ad $M, H, \tilde{M}_{V, k-i}$ le espressioni fornite dalle (4), (10), (9), utilizzando il n. 5, c'), e poi riduciamo i termini contenenti a fattore $V_{k}(A)$, i quali compaiono nei due membri con uguali coefficienti :

$$
\left(M^{1} M^{2} \ldots M^{s}\right)_{V}=P+Q
$$

ricaviamo un'uguaglianza del tipo:

$$
\sum_{i=1}^{k}\left(\mathrm{C}_{i} V_{k-i}(A)\right)_{V}=0,
$$

dove, per abbreviare, abbiamo posto:

(12) $\Gamma_{i}=(-1)^{i}\left(R^{[i]}\right)_{P}+\underset{i_{1}=0}{\stackrel{i}{i}} \underset{i_{2}=i_{1}}{\stackrel{i}{\Sigma} \ldots} \sum_{i_{s}=i_{s-1}}^{i}\left(\ldots\left[\left(Q_{V, i_{1}}^{i} \tilde{M}_{V, i_{2}-i_{1}}^{1}\right)_{M^{1}} \tilde{M}_{V, i_{3}-i_{2}}^{2}\right]_{M^{2}} \ldots \tilde{M}_{V, i-i_{s}}^{s}\right)_{M s}$.

$\mathrm{Se}$ - nelle considerazioni precedenti - si assume (come è lecito) $k=1$, la (11) si riduce a $\Gamma_{1}=0$; vogliamo dimostrare che ̀े

$$
\Gamma_{i}=0 \quad \text { per } i=1,2, \ldots, q .
$$

Fd invero, ammesso di aver stabilita la (13) per $i=1,2, \ldots, k-1$, la si ha subito per $i=k$ in forza della (11). Le (13) sono cosi dimostrate. Avuto riguardo alle (12), esse esprimono che è :

$$
\left.\left|Q^{0}\right|=\left(\ldots\left[\left|Q_{V} \| \tilde{M}_{V}^{1}\right|\right)_{M^{1}}\left|\tilde{M}_{V}^{2}\right|\right]_{M^{2}} \ldots\left|\tilde{M}_{V}^{s}\right|\right)_{M^{s}},
$$

dove $\left|Q^{0}\right|$ designi la successione di sostegno $Q \supset R$ data da:

$$
\left\{Q^{0}\right\}=Q, R,-\left(R^{[2]}\right)_{P},\left(R^{[3]}\right)_{P},-\left(R^{[4]}\right)_{P}, \ldots .
$$

Applicando ripetutamente la seconda proposizione del n. 8 , dalla (14) si trae la

$$
\left.\left\{Q_{V}\right\}=\left(\ldots\left[\left(\left|Q^{0}\right|\left\{M_{V}^{\mathrm{t}}\right\}\right)_{\boldsymbol{M}^{1}} \cdot\left\{M_{V^{\mathrm{a}}}^{\mathrm{a}}\right\}\right]_{\boldsymbol{M}^{\mathrm{s}}} \ldots \mid M_{V}^{\mathrm{s}}\right\}\right\}_{\boldsymbol{M}^{s}}
$$

Se consideriamo la successione $\left|P^{0}\right|$ di sostegno $P$ formata dalle

$$
P_{i}^{0}=(-1)^{i}\left(R^{[i]}\right)_{P} \quad(i=0,1,2, \ldots),
$$

e definiamo $N$ assumendo

$$
N=P+Q=\left(M^{1} M^{2} \ldots M^{s}\right)_{V},
$$

in base alla (15) - con ovvia notazione - risulta

$$
\left\{Q^{0}\right\}=-\left\{P^{0}\right\}+N \text {. }
$$


Sostituendo quest' espressione di $\left\{Q^{\prime}\right\}$ nella (16), e ricordando le (17), (18), $\left(\boldsymbol{2}_{38}\right)$, vediamo che,

Nelle ipotesi ammesse, le varietò $Q_{V}$; cercate sono fornite dalla

$$
\left.\left.\left.\left.\left.\mid Q_{V}\right\}=\left\{N_{V}\right\}-\left(\ldots\left[\left(\mid P^{0}\right\} \mid M_{V}^{1}\right\}\right)_{M^{1}} \cdot \mid M_{V}^{2}\right\}\right]_{M^{2} \ldots} \ldots M_{V}^{s}\right\}\right)_{M^{s}},
$$

$e$ ciò̀ si esprimono (per $i=0,1,2, \ldots$ ) con le equivalenze:

$$
\begin{aligned}
Q_{V, i} & =\sum_{i_{1}=0}^{i} \sum_{i_{2}=i_{1}}^{i} \ldots \sum_{i_{s-1}=i_{s-2}}^{i}\left(M_{V, i_{1}}^{1} M_{V, i_{2}-i_{1}}^{2} \ldots M_{V, i-i_{s-1}}^{s}\right)- \\
& -\sum_{i_{1}=0}^{i} \sum_{i_{2}=i_{1}}^{i} \ldots \sum_{i_{s}=i_{s-1}}^{\sum_{i}^{i}}(-1)^{i_{1}}\left(\ldots\left[\left(\left(R^{\left[i_{1}\right]}\right)_{P} M_{i_{2}-i_{1}}^{1}\right)_{M^{1}} M_{i_{3}-i_{2}}^{2}\right]_{M^{2}} \ldots M_{i-i_{3}}^{s}\right)_{M^{s}} .
\end{aligned}
$$
diventa

Così ad esempio, per $i=1$, la prima somma - avuto riguardo al n. $38-$

$$
N_{V, 1}=\sum_{l=1}^{s}\left(N M_{V, 1}^{l}\right)_{\boldsymbol{M}^{l}}
$$

e la seconda somma si spezza nelle due somme parziali dei termini provenienti dai valori 0 ed 1 dell'indice $i_{i}$, le quali rispettivamente valgono

$$
\sum_{l=1}^{s}\left(P M_{V, 1}^{l}\right)_{M^{i}} \quad \text { e }-R
$$

Tenuto conto delle (1), (18) e del n. 5, e'), abbiamo dunque:

$$
Q_{V, 1}=\sum_{l=1}^{s}\left(M^{1} \ldots M^{l-1} M_{V, 1}^{l} M^{l+1} \ldots M^{s}\right)_{V}+P_{V, 1}-2 \sum_{l=1}^{s}\left(P M_{V, 1}^{l}\right)_{M^{l}} .
$$

A questa stessa equivalenza si sarebbe anche potuto giungere più rapidamente, sottraendo a membro a membro dalla (1) la relazione che da essa si deduce con la sostituzione lecita delle $P, P_{V, 1}$ con le $Q, Q_{V, 1}$, e poi usufruendo della (18).

Osserviamo da ultimo che, avendo dianzi determinate le successioni covarianti $\left|R_{V}\right|$ e $\left|Q_{V}\right|$, se ne può trarre la $\left|R_{Q}\right|$, espressa con la

$$
\left|R_{Q}\right|=\left(\left|R_{V}\right| \cdot\left|\tilde{Q}_{V}\right|\right)_{Q}
$$

la quale subito segue da $R \subset Q \subset V$ in base al teorema dell' appartenenza (n. 18). Anoora più semplicemente si ha che, poichè $K$ è un'ipersuperficie di $Q$, in virtù del n. 14 risulta

$$
R_{Q, i}=(-1)^{i}\left(R^{[i+1]}\right)_{Q} \quad(i=0,1,2, \ldots) ;
$$

e questa fornisce le $R_{Q, i}$ quando si tenga conto della (1). Si noti però che tanto la (19) che le (20) fanno intervenire delle operazioni sopra la varieta $Q$, la quale non è stata direttamente assegnata. Nel numero successivo determineremo $\left\{R_{Q}\right\}$ sotto ipotesi più generali, in funzione unicamente delle $M, P$ e loro varietà covarianti, in modo da dover effettuare su queste soltanto operazioni entro gli anelli $\mathfrak{A}_{\mathcal{B}}, \mathfrak{z}_{P}, \mathfrak{A}_{V}$. 
40. Riferendoci ancora alle $s(\geq 2)$ varietà $M^{1}, M^{*}, \ldots, M^{s}$ situate in $V$ come nei nn. 9, 35, passiamo a considerare il caso generale in cui il numero $t$ dato dalla $\left(3_{35}\right)$ sia un intero (non negativo) qualsiasi. Proveremo che:

Per ogni $k$ soddisfacente alle $0 \leq k \leq v-p-1$ vale l'equivalenza:

$$
R_{Q, k}=P_{t+k+1}^{0}
$$

dove $t$ è l'intero suddetto $\left.e \mid P^{0}\right\}$ denota la successione di sostegno $P$ definita dalla $\left(1_{35}\right)$.

Osserviamo anzitutto che $i$ due membri della (1) hanno la stessa dimensione

$$
\dot{r}-k=p-(t+k+1)
$$

in forza della $\left(3_{35}\right)$ e della $r=q-1$ (n. 12). Basterà quindi stabilire la (1) per i valori $\mathrm{di} k$ in corrispondenza ai quali tale dimensione riesce non negativa, e cioè per $k \leq r$. Inoltre, poichè (n. 6) $R_{Q, 0}=R$, così la (1) sussiste per $k=0$ in conseguenza del n. 37. Potremo quindi, supposto $1 \leq k \leq r, k \leq v-p-1$, dimostrare la (1) ammettendo per induzione che valgano le

$$
R_{Q, 0}=P_{t+1}^{0}, R_{Q, 1}=P_{t+2}^{0}, \ldots, R_{Q, k-1}=P_{t+k}^{0} \text {. }
$$

E rileviamo che anche ora sussistono le $\left(20_{39}\right)$, giusta quanto si è detto alla fine del n. 39.

Scegliamo in $V$ genericamente $k+1$ ipersuperficie

$$
A^{1}, A^{2}, \ldots, A^{n+1}
$$

passanti per $P$. Poichè $v-k-1 \geq p$, così le (3) s'intersecano regolar. mente lungo una varieta

$$
M=\left(A^{3} A^{2} \ldots A^{k+1}\right)_{V}
$$

(di dimensione $v-k-1$ ), passante semplicemente per $P$. Inoltre, ciascuna delle (3) incontra $Q$ secondo $R$ e, ulteriormente, lungo una varietà di dimensione $q-1=r$ :

$$
Q^{i}=\left(Q A^{i}\right)_{V}-R \quad(i=1,2, \ldots, k+1) .
$$

Le (5) sono $k+1$ ipersuperficie di $Q$, la cui intersezione

$$
Q^{\prime}=\left(Q^{\prime} Q^{2} \ldots Q^{k+1}\right)_{Q}
$$

è una varietà pura di dimensione $q^{\prime}=q-k-1=r-\not \geq \geq 0$; ed è chiaro che questa non è altro che l'intersezione delle $s+1$ varietà $M^{ \pm}, M^{2}, \ldots, M^{s}, M$ residua a $P$. Relativamente ad essa, il numero $t^{\prime}$ analogo a $\left(3_{35}\right)$ vale

$$
t^{\prime}=p-q^{\prime}=p-q+k+1=t+k+1
$$

onde, applicando il teorema del n. 35, otteniamo:

$$
\begin{aligned}
& \left(M^{3} M^{\prime} \ldots M^{s} M\right)_{V}-Q^{\prime}= \\
& \text { (8) }=\sum_{i_{1}=0}^{t^{\prime}} \sum_{i_{2}=i_{1}}^{t^{\prime}} \ldots \sum_{i_{s}=i_{s-1}}^{t^{\prime}} \underset{i=i_{s}}{t^{\prime}}\left[\left(\ldots\left[\left(P_{V, i_{1}} \tilde{M}_{V, i_{2}-i_{1}}^{1}\right)_{M^{1}} \tilde{M}_{V, i_{3}-i_{2}}^{2}\right]_{M^{2}} \ldots \tilde{M}_{V, i-i_{s}}^{s}\right)_{M^{s}} \tilde{M}_{V, t^{\prime}-i}\right]_{M} .
\end{aligned}
$$


Si ricordi ora che, in virtù della (4), risulta (n. 17):

$$
\tilde{\mathbb{M}}_{V, i}=\left(M \cdot V_{i}(A)\right)_{V} \quad(i=0,1,2, \ldots),
$$

dove $V_{0}=V$ e $V_{j}(A)$ per $j=1,2, \ldots, k+1$ denota la funzione simmetrica elementare di grado $j$ delle (3), mentre $\otimes V_{j}=0$ per $j>k+1$. Avuto riguardo alla (7), si ha quindi che ciascuno dei termini provenienti dal secondo membro della (8) per $i=0,1, \ldots, t-1$ vale zero, sicchè nella (8) basta far assumere ad $i$ i valori interi da $t$ a $t^{\prime}=t+k+1$ (corrispondente ai quali $t^{\prime}-i$ decresce da $k+1$ allo zero). Poichè gli indici nella somma multipla che com. pare in (8) soddisfano alle

$$
i_{1} \leq i_{2} \leq i_{3} \leq \ldots \leq i_{s} \leq i,
$$

cosi, fissato in quella l'indice $i$ uguale ad uno dei suddetti valori, occorre limitare superiormente a tale valore la variabilità degli altri indici. Pertanto, tenuto conto delle $(4),(9)$, del n. $\left.5, c^{\prime}\right)$ e della $\left(1_{35}\right)$, posto $i=t+j$, la (8) può scriversi nella forma:

$$
\left(M^{1} M^{z} \ldots M^{s} \cdot V_{k+1}(A)\right)_{V}-Q^{\prime}=\sum_{j=0}^{k+1}\left(P^{0}{ }_{t+j} V_{k-j+1}(A)\right)_{V} .
$$

Esprimiamo da ultimo nella (6) le $Q^{\prime i}$ mediante le (5). Avuto riguardo alle (4), $\left(20_{99}\right)$ e rammentando che $\mathrm{e} R \subset Q$, otteniamo cosi :

$$
Q^{\prime}=\left(Q \cdot V_{k+1}(A)\right)_{V}-\sum_{j=1}^{k+1}\left(R_{Q, j-1} V_{k-j+1}(A)\right)_{V}
$$

Basta allora sostituire quest'espressione di $Q^{\prime}$ nella (10), ed usufruire delle (4), (2), per dedurre la (1).

Poichè $R \subset Q \subset V$, e tenuto conto del teorema dell'appartenenza (n. 18), note $\operatorname{cosi}$ le $R_{Q, k}$ se ne possono subito derivare le $R_{V, k}$, non appena si conoscano le $Q_{V, i}$.

Osservazione. - Il procedimento che testè ci ha condotti a stabilire la (1), può venir semplificato col sostituire al teorema del n. 37 ed al metodo d'induzione completa un principio d'identità fra polinomi in $\mathfrak{A}_{V}$, nel modo seguente. All' nopo si osservi che l'eliminazione di $Q^{\prime}$ fra le (10), (11) fornisce un' uguaglianza esprimente l'identita fra due polinomi, di grado $k+1$ nelle $A$. Tenuto conto della latitudine con cui possono venir scelte le $A$ (che sono ipersuperficie arbitrarie di $V$ passanti per $P$ ), se ne può trarre l'uguaglianza fra i coefficienti dei monomi simili nelle $A$, ciò che appunto porge la (1), assieme alle (2) ed alla $\left(1_{37}\right)$. La (1), precisamente, si ottiene uguagliando fra loro i termini di quei polinomi indipendenti dalle $A$, ed è quindi eiò ohe diventa l'identità suddetta quando vi si assuma ciascuna delle $A$ virtualmente nulla.

Un'analoga osservazione potrebbe altresi farsi relativamente agli sviluppi dei nn. 14, 15, 19, 39. 
41. Il problema di determinare $l e Q_{V, i}$ in funzione soltanto delle $M, N, P$ e loro varietà covarianti, al quale dianzi ci siamo ricondotti, presenta serie difficoltà nel caso generale. Esso è già stato da noi risolto nell'ipotesi che sia $P=0$ (n. 38) oppure $t=0$ (n. 39), ed ora lo risolveremo nel caso in cui $Q$ sia intersezione regolare di due date varietà $M, N d i V$ residua ad una varietà nota $P$ ad esse comune, supponendo:

$$
m \text { qualunque }(3 \leq m \leq v-1), \quad n=v-2, \quad p=q+1,
$$

e quindi :

$$
q=m+n-v=m-2, \quad p=m-1, \quad t=p-q=1 .
$$

A tal uopo, scegliamo in $V$ una generica ipersuperficie $A$ passante per $N$; questa segherà la $M$ secondo $P$ e, ulteriormente, lungo una varietà pura, $K$, di dimensione

(3)

Posto per abbreviare

$$
k=m-1=p .
$$

$$
P^{\prime}=(A M)_{V},
$$

le $P, K, P^{\prime}$ saranno tre ipersuperficie di $M$, ivi legate dall' equivalenza

$$
K=P^{\prime}-P
$$

(su $M$ ).

La $K$ suddetta e la $N$ sono manifestamente due varietà di $V$ aventi a comune la $Q$, lungo cui esse si intersecano regolarmente, essendo in virtù delle (1)-(3) :

$$
q=k+n-(v-1) \text {. }
$$

L'intersezione residua delle $K, N$ entro $A$ è una varietà pura di dimensione $q$. che denoteremo con $H$, la quale coincide con la varietà comune alle ipersuperficie $P$ e $K$ di $M$. Risulta pertanto

ed inoltre

$$
H=(P K)_{M},
$$

$$
Q=H^{\prime}-H,
$$

dove, per abbreviare, abbiamo posto

$$
H^{\prime}=(K N)_{A}
$$

Poichè $Q \subset K \subset M$, in forza del teorema dell'appartenenza (n. 18) si ha:

$$
Q_{M, i}=\sum_{j=0}^{i}\left(Q_{K, j} K_{M, i-j}\right)_{K} \text {. }
$$

Inoltre, in virtù del n. 14 valgono le

$$
Q_{M, j}=(-1)^{j}\left(Q^{[j+1]}\right)_{K}, \quad K_{M, i-j}=(-1)^{i-j}\left(K^{[i-j+1]}\right)_{M} .
$$

Sostituendo nella (9), ed usufrnendo del n. 5, c'), otteniamo:

$$
Q_{M}, i=(-1)^{i} \sum_{i=0}^{i}\left(\left(Q^{[j+1]}\right)_{K}\left(K^{[i-j]}\right)_{M}\right)_{M}
$$


Applicando il teorema dell'appartenenza alle varietà $N, A, V$ (di rispettive dimensioni $v-2, v-1, v$, abbiamo :

$$
\left|N_{A}\right|=\left(\left|N_{V}\right| \cdot\left|\tilde{A}_{V}\right|\right)_{A} .
$$

Inoltre, omettendo qui e nel seguito l'indice $V$ in basso per le moltiplicazioni da effettuarsi in $\mathfrak{A}_{V}$, ancora in base al n. 14 risulta

$$
N_{A, l}=(-1)^{l}\left(N^{[l+1]}\right)_{A}, \quad\left|\tilde{A}_{V}\right|=A, A^{[2]}, 0,0, \ldots .
$$

Si ha quindi

$$
(-1)^{l}\left(N^{[l+1]}\right)_{A}=N_{A, l}=N_{V, l}+N_{V, l-1} A .
$$

Notiamo poi che, in virtù della (6), e tenuto conto della $K \subset M$ e del n. 5, a') e della (5), risulta:

$$
\left(H^{[j+1]}\right)_{K}=\left(K P^{[j+1]}\right)_{M}=\left(P^{\prime} P^{[j+1]}\right)_{M}-\left(P^{[j+2]}\right)_{M} ;
$$

basta dunque valersi della (4) e del n. 5, a), per vedere che è :

$$
\left(H^{[j+1]}\right)_{K}=\left(P^{[j+1]}\right)_{M} A-\left(P^{[j+2]}\right)_{M} .
$$

Se $b \geq 0, c>0$ denotano due interi qualsiansi, avuto riguardo alla (6), alla $H^{\prime} \subset K$, ed al n. 5 , a), si perviene alla:

$$
\left(H^{[b]} H^{[c]}\right)_{K}=\left(P^{[b]}\left(H^{\prime}[0]\right)_{K}\right)_{M} \text {. }
$$

D'altro canto, tenendo conto delle (8), (5) ed usufruendo del n. 5, a'), si ricava:

$$
\left(H^{\prime}[c]\right)_{K}=\left(K N^{[c]}\right)_{A}=\left(P^{\prime} N^{[c]}\right)_{A}-\left(P N^{[c]}\right)_{A} .
$$

$\mathrm{E}$ siccome, in forza delle (4), (11) e del n. 5, a), si ha:

$$
\begin{gathered}
\left(P^{\prime} N^{[0]}\right)_{A}=M\left(N^{[c]}\right)_{A}=(-1)^{c-1} M\left(N_{V, c-1}+N_{V, c-2} A\right), \\
\left(P N^{[c]}\right)_{A}=\left(P\left(N^{[c+1]}\right)_{A}\right)_{N}=(-1)^{c}\left(P N_{V, c}\right)_{N}+(-1)^{c}\left(P N_{V, c-1}\right)_{N} A,
\end{gathered}
$$

così risulta:

$$
(-1)^{c-1}\left(H^{\prime c l}\right)_{K}=M N_{V, c-1}+M N_{V, c-2} A+\left(P N_{V, c}\right)_{N}+\left(P N_{V, c-1}\right)_{N} A .
$$

Osserviamo infine che, in virtù della (7), si ha

$$
(-1)^{j}\left(Q^{[j+1]}\right)_{K}=\sum_{c=0}^{j+1}(-1)^{o-1}\left(H^{[j-c+1]} H^{\prime[c]}\right)_{K}
$$

e da qui, in base alle (12), (13), (14), si deduce:

$$
\begin{aligned}
& (-1)^{j}\left(Q^{[j+1]}\right)_{K}=\sum_{c=1}^{j+1}\left(P^{[j-c+1]}\right)_{M}\left(N_{V, c-1}+N_{V, c-2} A\right)+ \\
& +\sum_{c=1}^{j+1}\left(\left(P N_{V}, d\right)_{N} P^{[j-c+1]}\right)_{M}+\sum_{c=1}^{j+1}\left(\left(P N_{V, c-1}\right)_{N} P^{[j-c+1]}\right)_{M} A+ \\
& +\left(P^{[j+2]}\right)_{M}-\left(P^{[j+1]}\right)_{M} A .
\end{aligned}
$$

Annali di Matematica 
Inoltre dalla (5), avuto riguardo alla (4), si trae

$$
\left(K^{[i-j]]_{M}}=\sum_{l=0}^{i-j}(-1)^{l}(i-j)\left(P^{[l]}\right)_{M^{A^{[i-j-l]}}}\right.
$$

Basta quindi sostituire i valori cosi ottenuti per $\left(Q^{[j+1]}\right)_{K}$ e $\left(K^{[i-j]}\right)_{M}$ nella (10), per dedurre un'espressione del tipo voluto per la $Q_{M, i}$. Tale espressione risulta indipendente da $A$, in conformità con l'osservazione finale del n. 40 ; fatte le debite riduzioni, e scritto $c+1$ in luogo di $c$, si perviene alla formula:

$$
\begin{aligned}
Q_{M, i} & =(i+1)\left(P^{[i+2]}\right)_{M}+ \\
& \left.+\sum_{c=0}^{\dot{\Sigma}}(i-c+1)\left[\left(P^{[i-c]}\right)_{M} N_{V, c}\right)_{V}+\left(\left(P N_{V, c+1}\right)_{N} P^{[i-c]}\right)_{M}\right]
\end{aligned}
$$

la quale rimane cosi stabilita per $i=0,1,2, \ldots$.

Possiamo agevolmente controllare la (15) nel caso più semplice $i=0$, in cui essa si riduce alla

$$
Q=(P[2])_{M}+(M N)_{V}+\left(P N_{V, 1}\right)_{N}
$$

mostrando come questa attualmente non differisca dalla formula

$$
Q=(M N)_{V}-P_{V, 1}-\left(P \tilde{M}_{V, 1}\right)_{M}-\left(P \tilde{N}_{V, 1}\right)_{N}
$$

fornita dai nn. 35, 36. Si ha intanto (n. 7):

$$
\tilde{N}_{V, 1}=-N_{V, 1}
$$

inoltre, in virtù del n. 14, ed applicando alle $P \subset M \subset V$ il teorema del l'appartenenza nolla forma $\left(4_{18}\right)$, otteniamo

$$
\left(P^{[2]}\right)_{M}=-P_{M, 1}=-P_{V, 1}-\left(P \tilde{M}_{V, 1}\right)_{M}
$$

sicchè la (16) equivale effettivamente alla (17).

La questione propostaci al principio del presente numero si risolve ormai in modo immediato. In virtù delle $Q \subset M \subset V$, si ha infatti (n. 18) :

$$
Q_{V, i}=\sum_{j=0}^{i}\left(Q_{M, j} M_{V, i-j}\right)_{M}
$$

e basta esprimere qui le $Q_{M, j}$ mediante le (15), per ottenere le $Q_{V, i}$ determinate nel modo voluto. 


\section{CAPITOLO QUARTO}

\section{ESTENSIONI A VARIETA SINGOLARI}

\section{§ I. - Intersezione di due o più ipersuperficie, residua ad una loro varietà multipla.}

42. Per ragioni di semplicità, le sottovarietà di $V$ considerate finora sono tutte state supposte (virtualmente) prive di punti multipli. Ci si può domandare in qual modo occorra modificare gli sviluppi precedenti qualora si lasci cadere quest'ipotesi. Una risposta esauriente a tale domanda esigerebbe un ampio complesso di ricerche, il cui compimento ci farebbe uscire dai confini segnati al presente lavoro. Ci limiteremo perciò a trattare qualche problema particolare in cui intervengono varietà singolari, il che basterà per mostrare quali accorgimenti tecnici convenga usare per affrontare simili questioni nel nuovo indirizzo.

Premettiamo un'utile convenzione algoritmica. Fissata una varietà $P$ non singolare di $V$, sappiamo (n. 13) come su essa si definisca la varieta covariante d'immersione $P_{V, i}$, di dimensione $p-i(i=0,1,2, \ldots)$. Ebbene, in conformità con gli sviluppi del n. 16, è utile talvolta considerare questa $P_{V, i}$ come una potenza simbolica di $P$ in $V$, di esponente

$$
j=v-p+i
$$

più precisamente, assumeremo per definizione

$$
[P]_{V}^{j}=P_{V, i},
$$

per tutte le coppie di interi $i, j$ soddisfacenti alla (1). E poichè i soli valori dell'indice $i$ per cui la $P_{V, i}$ non sia identicamente nulla sono quelli soddisfacenti alle limitazioni $0 \leq i \leq p$, così risulterà :

$$
[P]_{v}^{j}=0 \quad \text { tanto se } j<v-p \text { quanto se } j>v .
$$

Riferiamoci ora ad un polinomio simbolico in $P$, in cui $i$ coeffi. cienti siano varietă $M^{j}$ (virtuali) arbitrarie di $V$ :

$$
f(P)=\sum_{j=0}^{n} M^{j P^{[j]}}
$$

\section{Porremo per definizione}

$$
[f(P)]_{V}=f\left([P]_{V}\right)=\sum_{j=0}^{n} M^{j}[P]_{V}^{j},
$$

ossia in virtù delle (1), (2), (3), $[f(P)]_{V}=0$ se $n<v-p$, ed altrimenti

$$
[f(P)]_{V}=\sum_{j=v-p}^{n}\left(M^{j} P_{V, j+p-v}\right)_{V} ;
$$


è quindi chiaro che, se i coefficienti $M^{j}$ di $f(P)$ sono varietà pure di dimensione $r+j\left(\operatorname{con} r\right.$ ccstante), la $[f(P)]_{V}$ risulta una varietà pura di dimensione $r$.

Come conseguenza immediata della precedente definizione, si ha che l'operazione $[\ldots]_{V}$ è distributiva rispetto alla somma, e cioè risulta

$$
[f(P)+g(P)]_{V}=[f(P)]_{V}+[g(P)]_{V}
$$

Inoltre, con le convenzioni testè introdotte, la formula fondamentale $\left(4_{13}\right)$, che (nel n. 13) ci ha permesso di definire le varieta $P_{V, i}$, si riduce sim. bolicamente alla

$$
\begin{aligned}
& \left(A^{\mathrm{t}} A^{2} \ldots A^{s}\right)_{V}-\left(A^{1} A^{2} \ldots A^{s}\right)_{V}^{P}= \\
= & {\left[\left(A^{1}+P\right)\left(A^{2}+P\right) \ldots\left(A^{s}+P\right]_{V}\right.}
\end{aligned}
$$

qui, naturalmente, è inteso che il secondo membro abbia a calcolarsi serivendo anzitutto l'espressione entro parentesi quadre come un polinomio in $P$ della forma (4), e poi applicando la (5).

43. Sieno $M^{1}, M^{2}, \ldots, M^{s} s(\geq 2)$ varietà di $V$ passanti con date molteplicita $k_{1}, k_{2}, \ldots, k_{s}$ per una varietà irriducibile e non singolare $P$. Supporremo (come nel n. 9) che le dimensioni della varietà $M, P, V$ soddisfino alle

$$
p \geq m^{1}+m^{2}+\ldots+m^{s}-(s-1) v \geq 0 ;
$$

ammetteremo inoltre che le $M^{i}$ contengano $P$ con le assegnate molteplicita $k_{i}(\geq 1)$, senza presentare lungo $P$ ulteriori particolarità. Allora le $M^{4}, M^{2}, \ldots, M^{s}$ si segano secondo la $P$ (contata un certo numero di volte) ed inoltre lungo una varietà pura, $Q$, di dimensione

$$
q=m^{2}+m^{2}+\ldots+m^{s}-(s-1) v .
$$

Con argomentazioni consimili a quelle impiegate per dimostrare il teorema del n. 10, ed anche usufruendo con ovvie varianti del n. $16^{\prime}$ e di cio che s'è detto in nota nel n. 12, si prova che

Se le suddette varietà $M^{1}, M^{2}, \ldots, M^{s}, P$ variano su $V$ entro a sistemi d'equivalenza, in modo che le $\boldsymbol{M}^{i}$ continuino a passare genericamente per $P$ con le molteplicità $k_{i}$, anche l'intersezione $Q$ delle $M$ residua a $P$ varia entro ad un sistema d'equivalenza.

Quest' ultimo sistema d' equivalenza verrà denotato con

$$
Q=\left(\begin{array}{llll}
M^{1} M^{2} & \ldots & M^{s} \\
k_{1} & k_{2} & \ldots & k_{s}
\end{array}\right)_{V}^{P}
$$

E chiaro allora (n. 11) che l'equivalenza funzionale $E$ di $P$ nell'intersezione delle $M^{1}, M^{2}, \ldots, M^{s}$, quando queste abbiano a contenere genericamente $P$ con le molteplicita assegnate $k_{1}, k_{2}, \ldots, k_{s}$, si esprime mediante la

$$
E=\left(M^{i} M^{2} \ldots M^{s}\right)_{V}-\left(\begin{array}{llll}
M^{1} M^{2} & \ldots & M^{s} \\
k_{1} & k_{2} & \ldots & k_{s}
\end{array}\right)_{V}^{P}
$$


44. Risponderemo alla questione di determinare la varietà di cui è detto nel titolo del presente paragrafo, dimostrando che:

$L$ 'equivalenza funzionale $E$ di una varietà $P$ nell'intersezione di $s(\geq v-p)$ ipersuperficie $A^{1}, A^{2}, \ldots, A^{s} d i V$, quando queste siano soggette a contenere genericamente $P$ con date molteplicità $k_{1}, k_{2}, \ldots, k_{s}$, vale:

$$
E=\left[\left(A^{1}+k_{1} P\right)\left(A^{2}+k_{2} P\right) \ldots\left(A^{s}+k_{s} P\right)\right]_{V} .
$$

Il secondo membro di quest' equazione va calcolato in base alle convenzioni del $\mathrm{n} .42$, ed è pertanto una varieta pura di dimensione $v-s$ appartenente a $P$, ivi definita (a meno di un'equivalenza) non appena - oltre alle $A-$ si conoscano le varietà covarianti $P_{V, i}$; e si noti che l'ammessa limitazione $s \geq v-p$ non è che la forma attuale della $\left(1_{43}\right)$. Nel caso particolare di passaggi tutti semplici, e cioè se $k_{1}=k_{z}=\ldots=k_{s}=1$, la (1) si riduce manifestamente alla $\left(6_{4}\right)$, ed è quindi valida per definizione delle $P_{V, i}$.

All' nopo di stabilire la (1) negli altri casi, in virtù del n. $16^{\prime}$ è lecito assumere

$$
A^{i}=B^{i 1}+B^{i 2}+\ldots+B^{i k_{i}} \quad(i=1,2, \ldots, s),
$$

dove le $B$ sono ipersuperficie (virtuali) di $V$, passanti per $P$ semplice. mente ed in modo generico. Risulta allora (n. 3):

$$
\left(A^{1} A^{2} \ldots A^{s}\right)_{V}=\sum_{(j)}\left(B^{1 j_{1}} B^{2 j_{2}} \ldots B^{\left.s j_{s}\right)_{V}}\right.
$$

dove la somma va estesa a tutti i complessi $(j)=\left(j_{1}, j_{2}, \ldots, j_{s}\right)$ che si ottengono dando a $j_{i}$ i valori $1,2, \ldots, k_{i}$ (per $i=1,2, \ldots, s$ ). Inoltre, dalle (2) e dalla definizione data nel n. 43 per il simbolo $\left(2_{43}\right)$, si trae:

$$
\left(\begin{array}{llll}
A^{1} A^{2} & \ldots & A^{s} \\
k_{1} & k_{2} & \ldots & k_{s}
\end{array}\right)_{V}^{P}=\underset{(j)}{\Sigma}\left(B^{1 j_{1}} B^{2 j_{2}} \ldots B^{s j_{s}}\right)_{V}^{P}
$$

Basta quindi sottrarre le (3), (t) a membro a membro ed applicare le $\left(3_{4}\right)$, $\left(6_{49}\right)$, per dedurre l'equivalenza:

$$
E=\sum_{(j)}\left[\left(B^{ \pm j_{1}}+P\right)\left(B^{2 j_{2}}+P\right) \ldots\left(B^{s j_{s}}+P\right)\right]_{V} .
$$

In virtủ del n. 42, e poggiando sulle (2), ne consegue:

$$
\begin{aligned}
E & =\left[\sum_{(j)}\left(\left(B^{1 j_{1}}+P\right)\left(B^{2 j_{2}}+P\right) \ldots\left(B^{s j_{s}}+P\right)\right)\right]_{V}= \\
& =\left[\left(A^{1}+k_{1} P\right)\left(A^{2}+k_{2} P\right) \ldots\left(A^{s}+k_{s} P\right)\right]_{V} .
\end{aligned}
$$

45. Osserviamo infine come si possa estendere il significato delle $\left(2_{43}\right)$, $\left(3_{43}\right)$ al caso in cai qualeuno degli interi $k$ che ivi figurano sia nullo, assumendo ad esempio

$$
\begin{aligned}
\left(\begin{array}{cccc}
M^{1} M^{2} & \ldots & M^{s} \\
0 & k_{2} & \ldots & k_{s}
\end{array}\right)_{V}^{P} & =M^{1} \cdot\left(\begin{array}{lll}
M^{2} & \ldots & M^{s} \\
k_{2} & \ldots & k_{s}
\end{array}\right)_{V}^{P}= \\
& =\left(\begin{array}{cccc}
\left(M^{1} M^{2}\right)_{V} & \ldots & \left(M^{1} M^{s}\right)_{V} \\
k_{2} & \ldots & k_{s}
\end{array}\right)_{M^{1}}^{\left(M^{1} P\right)_{V}}
\end{aligned}
$$


Con cio, avuto anche riguardo al teorema della sezione (n. 19), ə̀ subito visto che:

Il teorema del $n$. 44 conserva la sua validità nell'ipotesi che taluna delle molteplicità $k$ sia uguale allo zero.

\section{§ II. - II luogo del punti doppi Impropri d' una varietà subordinata di $V$.}

46. Sia $P$ una varieta irriducibile di $V$, di dimensione abbastanza elevata rispetto a quella dj $V$, e precisamente tale che si abbia

$$
2 p \geq v \text {. }
$$

Si vede allora che, se si vuole che $P$ - come varietà del proprio ordine appartenente a $V$ - risulti a moduli generali; occorre supporre che $P$ contenga dei punti doppi impropri, costituenti una varietà algebrica pura $D$, di dimensione

$$
d=2 p-v\left({ }^{(4)}\right)
$$

Cio accade p. es. quando, $V$ essendo uno spazio lineare $S_{v}$, si ammette che $P$ possegga cosidette singolarità ordinarie (del tipo cioè di quelle che acquista la proiezione di una varietà $P^{*}$ non singolare di $S_{2 p+1}$, quando si proietti $P^{*}$ genericamente su di un $S_{v}$ subordinato di $S_{2 p+1}$ ).

Supponiamo dunque che $P$ contenga un luogo $D$ siffatto di punti doppi impropri, la cui dimensione $d$ soddisfi alla (2), e ohe ogni punto di $P$ non appartenente a $D$ sia semplice per $P$. Cio non significa naturalmente che $P$ non possa contenere singolarità di tipo più elevato; ma soltanto che il luogo di queste ultime (se esistono) abbia a costituire un certo numero di sottovarieta di $D$, ond'esso verrà ad avere dimensione inferiore a $d$. Pertanto il generico punto di $D$ risulta un punto doppio improprio di $P$ del tipo più generale, sicchè - dal punto di vista della geometria sopra la varietà $P$ - esso deve considerarsi come la sovrapposizione di due punti semplici di $P$. Corrispondentemente, $D$ - pensata quale varietà sopra $P$ - dovrà valutarsi, su $V$, come equivalente al doppio della varietà algebrica semplice lnogo dei suoi punti; per evitare equivoci, questa varietà di $V$ verrà denotata con $(D)$, e potremo così scrivere l'equivalenza:

$$
D=2(D) \quad \text { (su } V) \text {. }
$$

Indicheremo poi con $(P)$ la varietà $P$ in cui il luogo singolare $D$ si consideri come virtualmente inesistente. Così, ad esempio, se in un sistema di equivalenza contenente totalmente $P$ esiste qualche varietì priva di punti multipli, ognuna di queste può venir assunta come un rappresentante di $(P)$, ed in tal caso $P$ può ottenersi come posizione limite della varietà non

(14) Cfr. SeverI [1], § 2. 
singolare $(P)$. In ogni caso, varrà ovviamente l'equivalenza

$$
P=(P)
$$

(su $V$ ).

Un' ipersuperficie $A$ di $V$ passante genericamente per $P$ viene necessariamente ad acquistare $D$ come luogo di punti doppi ordinari. Denoteremo similmente con $(A)$ l'ipersuperficie $A$ considerata come virtualmente priva di punti multipli, e quindi passante virtualmente con molteplicita 1 anche per $D$.

Con le suddette locuzioni, ed avuto riguardo al n. 13 , si ha senz'altro cio che deve intendersi per le varietà covarianti $P_{V, i}$ e $(P)_{V, i}$ rispettivamente di $P \Theta(P)$ in $V$. Ed è chiaro che, fintantochè la dimensione $p-i$ di queste varietà si mantiene superiore alla dimensione $d$ di $D$, il procedimento del n. 13 non subisce alterazioni per effetto della varietà doppia $D$ di $P$. Poichè, in forza della (2), la limitazione $p-i>d$ equivale alla $i \leq v-p-1$, cosi intanto s a $P$ risulta

$$
P_{V, i}=(P)_{V, i} \quad \text { per } i=0,1, \ldots, v-p-1 .
$$

Si ha inoltre che la (5) [la quale si riduce per $i=0$ alla (4)] dovrà ge* neralmente cessare di sussistere a partire dal valore $i=v-p$ dell' indice $i$; vedremo nel numero successivo com'essa debba venir modificata per $i=v-p\left({ }^{15}\right)$. Ora aggiungiamo soltanto che, in forza del n. 7, dalle (5) discendono immediatamente le

$$
\tilde{P}_{V, i}=(\tilde{P})_{V, i} \quad \text { per } i=0,1, \ldots, v-p-1 .
$$

47. Per rispondere alla questione posta or ora, introduciamo l'intero

$$
s=2 v-2 p,
$$

ed osserviamo ch esso soddisfa alle limitazioni $\left(1_{13}\right)$, in forza della $\left(1_{46}\right)$; corrispondentemente, il numero $t$ definito dalla $\left(2_{13}\right)$ vale:

$$
t=v-p \text {. }
$$

Possiamo cosı considerare $s$ generiche ipersuperficie

$$
A^{2}, A^{2}, \ldots, A^{s}
$$

di $V$ passanti per $P$ : l'intersezione $Q$ di queste residna a $P$ ha dimensione

$$
q=v-s=2 p-v=d,
$$

e - giusta i nn. 9, 46 - può venir denotata con $\left(A^{1} A^{2} \ldots A^{s}\right)_{V}^{P}$. L'analoga varietà $\left(A^{1} A^{2} \ldots A^{s}\right)_{V}^{(P)}$, che si ottiene in simil guisa a partire dalla $P$ considerata come virtualmente priva di punti maltipli, risulta equivalente alla somma di $Q$ con la varietà $D$ (n. 46), ossia

$$
\left(A^{4} A^{2} \ldots A^{s}\right)_{V}^{(P)}=\left(A^{1} A^{2} \ldots A^{s}\right)_{V}^{P}+D .
$$

(15) L' analoga questione per i valori dell'indice $i>v-p$ è assai più complessa, portando a dover considerare i vari luoghi dei punti di $D$ ove $P$ possiede una singolarità di tipo più elevato di quella che $P$ armette nel generico punto di $D$. 
In base ai nn. 13,46 , valgono tanto la $\left(4_{13}\right)$ quanto la relazione che da essa si ottiene scrivendo dappertutto $(P)$ in luogo di $P$, e quindi $(P)_{V, i}$ in luogo di $P_{V, i}$. Basta quindi sottrarre queste due relazioni a membro a membro, e ricordare le (1), (2), $\left(5_{46}\right)$, per ottenere la richiesta equivalenza:

$$
P_{V, v-p}=(P)_{V, v-p}+D \text {. }
$$

48. Possiamo dare all'ultima relazione forma più significativa, osservando che, sottraendo a membro a membro le equivalenze

$$
\sum_{i=0}^{v-p} P_{V, v-p-i} \tilde{P}_{V, i}=0, \quad \sum_{i=0}^{v-p}(P)_{V, v-p-i}(\tilde{P})_{V, i}=0,
$$

fornite dal n. 7 , ed applicando le $\left(5_{46}\right),\left(6_{46}\right)$, otteniamo:

$$
P_{V, v-p}+\tilde{P}_{V, v-p}=(P)_{V, v-p}+(\tilde{P})_{V, v-p} \text {. }
$$

In virtù di quest' ultima, la $\left(3_{47}\right)$ diventa:

$$
D=(\tilde{P})_{V, v-p}-\tilde{P}_{V, v-p} \text {. }
$$

Infine, poichè in base al $\mathrm{n} .23$ risulta

$$
\left(\tilde{P}_{V, v-p}=\left(P^{[2]}\right)_{V},\right.
$$

perveniamo così all'equivalenza

$$
D=\left(P^{[2]}\right)_{V}-\tilde{P}_{V, v-p},
$$

esprimente il luogo dei punti doppi impropri di $P$ come differenza fra la prima varietà caratteristica di $P$ in $V$ e la varietà covariante $\tilde{P}_{V, v-p}\left({ }^{16}\right)$.

Tale differenza è nulla se $P$ non possiede punti multipli, ed allora si ricade nel teorema del n. 23. Si rilevi poi che le $\left(3_{47}\right),(1)$ possono pensarsi come equivalenze su $P$, nel qual caso la varietà doppia $D$ dev'essere intesa nel modo specificato nel $n$. 46 ; mentre invece, qualora si vogliano ottenere delle equivalenze su $V$, converrà esprimere in esse $D$ mediante la $\left(3_{46}\right)$.

\section{§ III. - Sul luogo dei punti singolari imposti ad una varietà dal passaggio per un'altra,}

49. Assegnata in $V$ una varietà irriducibile $P$, si consideri una qualunque sottovarietà $M$ di $V$ passante per $P$, la cui dimensione $m$ soddisfi alle limitazioni

$$
p<m \leq 2 p-1 \quad(m<v)
$$

(16) In base a risultati che verranno stabiliti nel Cap. $7^{\circ}$, si potrebbe dimostrare la sostanziale equivalenza fra la formula (1) del testo e la (7.01) in ToDD [8]. Casi particolari della formula suddetta erano stati precedentemente ottenuti da ThuLLEN [1], B. SEgRE [4], Yoxalt [1]. 
(implicanti la $p \geq 2$ ). Se, essendo $2 p \geq v, P$ ammette un luogo $D$ di punti doppi impropri avente dimensione regolare (n. 46) :

$$
d=2 p-v
$$

anche $M$ dovrà passare doppiamente per $D$; il luogo $D$ viene naturalmente a mancare quando $P$ sia priva di punti multipli, il che sempre supporremo che accada se $2 p<v$, E interessante rilevare che in ogni caso, in forza delle (1), $M$ viene generalmente a dover avere su $P$ dei punti multipli fuori di $D$, e ciò in punti semplici di $P$. Mostreremo infatti, con un conto di costanti, che:

Se valgono le (1), la varietà $M$ - in conseguenza del suo passaggio per $P-$ deve generalmente acquistare un luogo $\Delta$ di punti doppi, contenuto semplicemente in $P$ ed avente dimensione

$$
\delta=2 p-m-1\left({ }^{17}\right) \text {. }
$$

All'uopo trasformiamo $V$ in una varietà $V^{*}$ di ugual dimensione, mediante una dilatazione di base $P\left({ }^{18}\right)$. Questa muterà i punti $O$ di una conveniente porzione $p$-dimensionale di $P$ in $\infty^{p}$ spazi lineari, $O^{*}$, di dimensione $v-p-1$. Ciasouno di questi ultimi potrà venir considerato come luogo del punto

$$
x=\lambda_{1} x^{1}+\lambda_{2} x^{2}+\ldots+\lambda_{v-p} x^{v-p},
$$

dove le $\lambda$ sono parametri ed $x^{1}, x^{4}, \ldots, x^{v-p}$ denotano $v-p$ punti indipendenti di $O^{*}$, funzioni analitiche regolari di $p$ parametri $\mu_{i}, \mu_{2}, \ldots, \mu_{p}$, fissanti la posizione di $O$ entro quella porzione di $P$.

Detta $M^{*}$ la varietà trasformata di $M$, il fatto che $M$ passi semplicemente per $O$ si traduce in ciò che $M^{*}$ incontra $O^{*}$ lungo uno spazio lineare subordinato, di dimensione $m-p-1$. Quest'ultimo potrà quindi venire rappresen. tato con un sistema di $v-m$ equazioni lineari indipendenti nelle $\lambda_{1}, \lambda_{2}, \ldots, \lambda_{v-p}$, a coefficienti funzioni analitiche dei parametri $\mu_{1}, \mu_{2}, \ldots, \mu_{p}$. Scrivendo che la matrice di tali coefficienti ha rango inferiore a $v-m$, si vengono ad imporre alle $\mu$

$$
v-p-(v-m-1)=m-p+1
$$

condizioni distinte; sicchè, in generale, vi saranno $\infty^{p-(m-p+1)}=\infty^{\delta}$ valori delle $\mu$ che le soddisfano. Questi proverranno da punti generalmente semplici di $P$, per oiascuno dei quali la $M$ dovrà passare (almeno) doppiamente. Il luogo $\Delta$ - necessariamente algebrico - costituito dai punti semplici di $P$ doppi per $M$ e dai loro punti di accumulazione, dovrà dunque avere di fatto la dimensione (3).

Abbiamo gia osservato che, se $2 p \geq v$, la varietà $P$ - pur essendo in un certo senso generica - può ammettere un luogo $D$ di punti doppi impropri,

(17) Questo risultato trovasi già stabilito in SEverr [1], $\$ 3$, nell'ipotesi che $V$ sia uno spazio lineare $\theta$ che tanto $P$ quanto $M$ risultino intersezioni complete di forme.

(i8) Ofr. B. SEgre [6], \$1. 
avente la dimensione (2), il quale allora risulterà anche doppio per M. Rileviamo che, in base alle (2), (3), se $M$ è un'ipersuperficie di $V(m=v-1), D$ ha la stessa dimensione $\delta$ di $\Delta$; negli altri casi $(m<v-1), D$ ha sempre dimensione inferiore $a \delta$. Poichè la varietà dei punti multipli di $M$, giusta quanto precede, dev' essere generalmente pura, così

Se $2 p \geq v$, l' eventuale varietà doppia impropria $D$ di $P$ apparliene generalmente a $\Delta$; le cose vanno però diversamente se $M$ è un'ipersuperficie di $V$, nel qual caso le due varietà $D$ e $\Delta$ hanno ugual dimensione e risultano in generale distinte.

50. A complemento delle precedenti considerazioni, dimostreremo ora il

Teorema. - Se valgono le $\left(1_{49}\right)$ ed è inoltre $m<v-1$, la varietà $\Delta d i$ cui al n. 49 - come varietà semplice di $P$ - vien fornita dall' equivalenza

$$
\Delta=\tilde{P}_{M, m-p+1} \text {. }
$$

Nell'ipotesi che invece si abbia $2 p>m=v-1$, la (1) dev'esser sostituita dalla

$$
\Delta=\tilde{P}_{M, v-p}-D,
$$

dove $D$ denota il luogo dai punti doppi impropri di $P$ (da pensarsi ciascuno come la sovrapposizione di due punti di P) e può quindi venir espresso mediante la $\left(1_{48}\right)$.

Introduciamo una varietà $U$ di dimensione $u=m+1$, che dovrà intendersi coincidente con $V$ nel secondo dei due casi contemplati nel teorema, mentre invece, nel primo caso, $U$ sarà una generica sottovarietà di $V$ avente dimensione $m+1$ e passante per $M$. A norma del n. 49 , la $\dot{U}$ potrà avere su $P$ un luogo singolare, di dimensione $2 p-m-2$; tuttavia, poichè questo numero risulta inferiore al numero $\delta$ espresso dalla $\left(3_{49}\right)$, così una siffatta singolariț non darà alcun disturbo nello studio del luogo $\Delta$ (di dimensione $\delta$ ), ossia - agli effetti di tale studio - potremo supporre la $U$ virtualmente priva $d i$ punti multipli.

Sia $P^{*}$ una varietà equivalente a $P$ ed appartenente ad $M$. In base ni nn. $48,49, M$ dovrà avere un luogo $\Delta^{*}$ di punti doppi impropri in punti generalmente semplici di $P^{*}$ ed inoltre, nel secondo dei due casi suddetti, $P^{*}$ dovrà avere un luogo $D^{*}$ di punti doppi impropri, pure doppi per $M$; poichè $M$ non ha generalmente punti singolari fuori di $P$, cosi $\Delta^{*}$ e $D^{*}$ debbono rispettivamente coincidere con $\Delta$ e $D$, ossia $P^{*}$ deve passare semplicemente per $\Delta$ e doppiamente per $D$. Consideriamo poi in $U$ una varietà $P^{\prime}$ equivalente a $P$ e non giacente in $M$, e denotiamo con $M^{\prime}$ una varieta di $U$ equivalente ad $M$ e passante per $P^{\prime}$. La varietà $M^{\prime}$ possiederà un luogo $\Delta^{\prime}$ di punti doppi in punti generalmente semplici di $P^{\prime}$, e (nel secondo di quei due casi) un luogo $D^{\prime}$ di punti doppi tanto per essa che per $P^{\prime}$. È chiaro che, quando $M^{\prime}$ - variando su $U$ entro ad un sistema d'equivalenza - tende ad $M$, le varieta $\Delta^{\prime}, D^{\prime}$ tendono rispettivamente alle $\Delta, D$; sicchè, per una nota proprietà in- 
finitesimale, può dirsi che la varietà $M^{\prime}$ - quand'è infinitamente prossima alla $M$ - passa semplicemente per le $\Delta, D$.

In virtù del n. 5, b) e c), fra le anzidette varietà intercedono le relazioni

$$
\begin{gathered}
\left\langle P P^{\prime}\right\rangle=\left(\left(P M^{\prime}\right)_{U}\left(P^{\prime} M\right)_{U}\right)_{\left(M M^{\prime}\right)_{U}}, \\
\left\langle\left(P P^{*}\right) M^{\prime}\right\rangle=\left(\left(P M^{\prime}\right)_{U}\left(P^{*} M^{\prime}\right)_{U}\right)_{\left(M M^{\prime}\right) U},
\end{gathered}
$$

ogni membro delle quali è una varietà di dimensione $\delta$; ne consegue la

$$
\left\langle P P^{\prime}\right\rangle=\left\langle\left(P P^{*}\right) \boldsymbol{M}^{\prime}\right\rangle
$$

Effettuiamo ora un passaggio al limite, facendo tendere $P^{*}$ e $P^{\prime}$ a $P$, ed $M^{\prime}$ ad $M$. Si ha intanto

$$
\lim \left\langle P P^{\prime}\right\rangle=\left(P^{[2]}\right)_{U}, \quad \lim \left\langle P P^{*}\right\rangle=\left(P^{[2\}}\right)_{M} .
$$

Relativamente al secondo limite, va però rilevato che, mentre la $\left(P P^{*}\right)$ passa semplicemente per $\Delta$ e - se $m=v-1$ - anche per $D$, la varieta $\left(P^{[:]}\right)_{M}$ deve ritenersi virtualmente non passante per le $\Delta, D$, in quanto queste sono varietà doppie di $M$. Se ne trae che è

$$
\lim \left\langle\left(P P^{*}\right\rangle M^{\prime}\right\rangle=\left(\left(P^{[2]}\right)_{M} M\right)_{U}+\Delta+D,
$$

ove - a norma di ciò che precede - il termine in $D$ va soppresso se $m<v-1$. Dalla (3) si ha quindi l'equivalenza

$$
\Delta=\left(P^{[2]}\right)_{U}-\left(\left(P^{[2]}\right)_{M} M\right)_{U}-D,
$$

con la medesima convenzione relativamente a $D$.

Poichè le varietà singolari di $M$ ed $U$ hanno dimensioni rispettivamente inferiori a quelle delle prime varietà caratteristiche di $P$ in $M$ ed $U$, cosi possiamo applicare il teorema del n. 23, deducendo per queste le equivalenze

$$
\left(P^{[2]}\right)_{U}=\tilde{P}_{U, m-p+1}, \quad\left(P^{[2]}\right)_{M}=\tilde{P}_{M, m-p} .
$$

Per analoghí motivi è lecito applicare al caso attuale la $\left(5_{t 8}\right)$ ed il teorema del n. 14, il che fornisce

$$
\tilde{P}_{U, m-p+1}=\sum_{i=0}^{m-p+1}\left(\tilde{P}_{M, m-p-i+1} \tilde{M}_{U, i}\right)_{M}=\tilde{P}_{M, m-p+1}+\left(\tilde{P}_{M, m-p} M\right)_{U}
$$

In base a questa ed alle (5), la (4) conduce senz'altro alle formule (1) e (2) che dovevano stabilire.

51. I risultati del n. 50 possono venir posti sotto forma equivalente, ma più comoda per le applicazioni, utilizzando il n. 48 e la $\left(6_{4,8}\right)$. Quest'ultima porge :

$$
\tilde{P}_{M, m-p+1}=\sum_{i=0}^{m-p+1}\left(\hat{P}_{V, m-p-i+1} M_{V, i}\right)_{M}
$$


sicchè, per $m=v-1$, risulta :

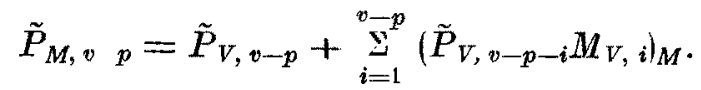

Usufruendo allora della $\left(1_{48}\right)$, si vede che:

$L a$ varietà $\Delta$, di cui il $n$. 50, se $m<v-1$ è data dalla

$$
\Delta=\sum_{i=0}^{m-p+1}\left(\tilde{P}_{V, m-p-i+1} M_{V}, i\right)_{M}
$$

mentre se $m=v-1$ si esprime con la

$$
\Delta=2 \tilde{P}_{V, v-p}-\left(P^{[?]}\right)_{V}+\sum_{i=1}^{v-p}\left(\tilde{P}_{V, v-p-i} M_{V, i}\right)_{M}
$$

\section{CAPITOLO QUINTO}

\section{SU1 PRODOTTI DIRETTI DI DUE O PIÙ VARIETA ALGEBRICHE}

\section{§. - Definizioni e prime proprietà.}

52. Si perviene, come mostreremo, a dare una portata assai più ampia a vari dei precedenti risultati ed a trarre da essi conseguenze d'un nuovo tipo, con l'uso delle cosidette varieta prodotto. Per chiarezza esporremo nel presente Capitolo le proprietà di tali varietà che ci occorrerrano in seguito, pur avvertendo che varie di esse possono considerarsi come note $\left({ }^{19}\right)$.

Date $s(\geq 2)$ varietà algebriche (semplici) qualsiansi :

$$
V^{1}, V^{2}, \ldots, V^{s}
$$

si può costruire - p. es. nel modo che indichiamo più sotto - una varietà algebrica, $V$, luogo di un punto $x$ che corrisponda algebticamente e biunivocamente alle $s$-ple ordinate di punti $x^{1}, x^{2}, \ldots, x^{\varepsilon}$ tratti rispettivamente dalle $V^{\prime}, V^{z}, \ldots, V^{s}$. La varietà $V$ risulta determinata a meno di una trasformazione birazionale senza eccezioni, e si chiama il prodotto diretto (taluni usano invece l'attributo di topologico), o anche semplicemente il pródotto, delle varietà (1). La relazione fra la $V$ e queste ultime e quella fra il punto $x$ e la corrispondente $s$-pla $x^{1}, x^{2}, \ldots x^{s}$ si usano esprimere simbolicamente con le scritture:

$$
V=V^{1} \times V^{2} \times \ldots \times V^{s}
$$

$$
x=x^{1} \times x^{2} \times \ldots \times x^{s} .
$$

(10) Per talune di esse ed altre proprietà, cfr. Severi [8], Hodge e PeDoe [1] Cap. XI e XII, Gamta [1]. Nel primo e nel terzo di questi lavori trovansi anche indicazioni biblio. grafiche ulteriori sull'argomento. 
5:3. Il più semplice modello proiettivo del prodotto di s spazi lineari $S_{c_{1}}^{1}, S_{c_{3}}^{z}, \ldots, S_{c_{s}}^{s}$, fu assegnato da CoRRado SEgRE come segue. Si introducano in $S_{c_{i}}^{i}$ coordinate proiettive omogenee di punto

$$
x_{0}^{i}, x_{1}^{i}, \ldots, x_{c_{i}}^{i}
$$$$
(i=1,2, \ldots, s)
$$

e si consideri uno spazio lineare $S_{d}$, di dimensione

$$
d=\left(c_{1}+1\right)\left(c_{2}+1\right) \ldots\left(c_{s}+1\right)-1,
$$

nel quale le coordinate proiettive omogenee vengano denotate da simboli $x_{j_{1} j_{2} \ldots j_{s}}$, che dipendano da $s$ indici $j_{i}$ assumenti $\mathrm{i}$ valori $j_{i}=0,1, \ldots, c_{i}$ $(i=1,2, \ldots, s)$. Allora la varietà di Segre

$$
T=S_{c_{1}}^{1} \times S_{c_{2}}^{2} \times \ldots \times S_{c_{s}}^{s}
$$

è la varietà rappresentata parametricamente in $S_{d}$ dalle equazioni

$$
x_{j_{1} j_{2} \ldots j_{s}}=x_{j_{1}}^{1} x_{j_{3}}^{2} \ldots x_{j_{s}}^{s} \quad\left(j_{i}=0,1, \ldots, c_{i} ; i=1,2, \ldots, s\right) .
$$

Si dimostra facilmente che $T$ è una varietà irriducibile, anzi birazionale, di dimensione $c_{1}+c_{2}+\ldots+c_{s}$ ed ordine

$$
\frac{\left(c_{1}+c_{2}+\ldots+c_{s}\right) !}{c_{1} ! c_{2} ! \ldots c_{s} !},
$$

mutata in sè da un grappo transitivo di omografie, imagini delle $s$-ple di sostitazioni lineari invertibili sulle $s$ serie di variabili (1). Da qui segue che $T$ risulta priva di punti multipli. Una dimostrazione di quest'ultimo fatto, e di taluno di quelli precedentemente enunciati, può venir presentata così.

Sia $x$ un qualunque punto di $T$, proveniente dalla $s$-pla di punti $x^{1}, x^{2}, \ldots, x^{s}$ degli spazi $S_{c_{1}}^{1}, S_{c_{2}}^{2}, \ldots, S_{c_{s}}^{s}$. Disponendo, se necessario, di opportune sostituzioni lineari sulle (1), possiamo ridurci ad avere in quei punti, e quindi altresi in certi loro intorni,

$$
x_{0}^{1}=x_{0}^{2}=\ldots=x_{0}^{s}=1, \quad \text { eppertanto } x_{00 \ldots 0}=1 .
$$

Allora le $d$ coordinate $x_{j_{1} j_{2} \ldots j_{s}}$ aventi le $j$ non tutte nulle sono coordinate proiettive non omogenee di punto in $S_{d}$, conservantisi finite in un intorno del punto $x$ su $T$; e precisamente, a norma delle $(3), c_{1}+c_{2}+\ldots+c_{s}$ di quelle $x_{j_{1} j_{2} \ldots j_{s}}$ uguagliano rispettivamente i parametri non omogenei

$$
x_{1}^{1}, \ldots, x_{c_{1}}^{4} ; x_{1}^{2}, \ldots, x_{0}^{2} ; \ldots ; x_{1}^{s}, \ldots, x_{c_{s}}^{s},
$$

e le altre sono dei polinomi in questi ultimi. Ciò dimostra che $x$ è punto semplice di $T$, e che $T$ ha di fatto dimensione $c_{1}+c_{2}+\ldots+c_{s}$. 
54. Possiamo ora costruire - come mostreremo - un modello proiettivo della più generale varietà prodotto $\left(2_{52}\right)$. Introdotte nello spazio $S_{e_{i}}^{i}$ ambiente di $V^{i}$ coordinate proiettive omogenee $\left(1_{53}\right)$, si consideri la varietà di Segre $\left(2_{53}\right)$ rappresentata dalle $\left(3_{53}\right)$. Il modello in questione è il luogo descritto dal punto

$$
x=x^{1} \times x^{2} \times \ldots \times x^{s}
$$

di quest' ultima, quando $x^{1}, x^{2}, \ldots, x^{s}$ descrivano rispettivamente le varietà $V^{1}, V^{2}, \ldots, V^{s}$. Ciò val quanto dire che le equazioni di $V$ si ottengono aggregando alle $\left(3_{53}\right)$ le equazioni nelle $x^{i} j_{i}$ che rappresentano $V^{i}$ nello spazio $S_{c_{i}}^{i}$ (per $i=1,2, \ldots, s)$.

Si vede facilmente che, se le $V^{1}, V^{*}, \ldots, V^{s}$ sono varieta pure, di dimensioni $v^{1}, v^{2}, \ldots, v^{*}$, anche la $V$ risulta pura, di dimensione $v=v^{1}+v^{2}+\ldots+v^{s}$; e che $V$ e irriducibile e non singolare se, e soltanto se, rispettivamente $\dot{e}$ tale ciascuna delle $V^{1}, V^{2}, \ldots, V^{s}$. Ad esempio, il fatto che il punto (1) risulta semplice per $V$ nell'ipotesi che le $V^{1}, V^{2}, \ldots, V^{r s}$ abbiano un punto semplice rispettivamente in $x^{4}, x^{2}, \ldots, x^{s}$, si dimostra nel modo seguente.

Si può intanto supporre, come nel n. 53, che entro opportuni intorni dei punti $x^{1}, x^{2}, \ldots, x^{s}, x$ sulle $V^{1}, V^{2}, \ldots, V^{s}, V$ ralgano le $\left(4_{53}\right)$. Allora l'ipotesi ammessa si traduce in ciò che, in un intorno del punto $x^{i}$ sulla $V^{i}$, certe $v^{i}$ fra le coordinate non omogenee $x_{1}^{i}, \ldots, x_{c_{i}}^{i}$ possano venir assunte come parametri uniformizzanti. Ma allora, tenuto conto del n. 53, si trae subito che, in un intorno del punto $x$ su $V$, certe $v=v^{1}+v^{2}+\ldots+v^{s}$ fra le coordinate non omogenee $x_{j_{1} j_{2} \ldots j_{s}}$ fungono da parametri uniformiz. zanti; e tanto basta per poter asserire che il punto $x$ è semplice per $V$, e che la dimensione di $V$ nell' intorno del punto $x$ vale $v$.

55. Conservando le notazioni dei nn. 52, 54, supporremo le $V^{i}$ pure e non singolari, sicchè tale risulterà altresì la $V$. Presa una qualunque sottovarietà $M^{i}$ di $V^{i}$, definiamo la sottovarietà $M^{\prime t}$ di $V$ assumendo

$$
M^{i}=V^{1} \times \ldots \times V^{i-1} \times M^{i} \times V^{i+1} \times \ldots \times V^{s} \quad(i=1,2, \ldots, s) ;
$$

in base al n. 54, la (1) è pura e priva di componenti multiple se tale è $M^{i}$, ed ha in ogni caso la dimensione

$$
m^{i}=v^{1}+\ldots+v^{i-1}+m^{i}+v^{i+1}+\ldots+v^{s}=v+m^{i}-v^{4} .
$$

In particolare, se $M^{t}$ è un' ipersuperficie di $V^{t}$, anche $M^{\prime i}$ risulta un'ipersuperficie di $V$; inoltre è subito visto che, se $M^{i}$ descrive su $V^{i}$ un sistema lineare, anche $M^{\prime i}$ varia su $V$ entro ad un sistema lineare. Da qui, tenuto conto del n. 2, si deduce più generalmente che:

Se $M^{i}$ descrive su $V^{i}$ un sistema elementare effettivo od un sistema d'equivalenza, anche $M^{\prime i}$ varia su $V$ rispettivamente entro ad un sistema elementare effettivo o ad un sistema d' equivalenza. 
Dalla definizione delle varietà (1) si ha poi subito che l'interferenza delle varietà

$$
M^{\prime 1}, M^{\prime 2}, \ldots, M^{\prime s}
$$

non è altro che il luogo dei punti giacenti sulla sottovarietà di $V$ che - a norma del n. 52 - può venir denotata col simbolo

$$
M^{1} \times M^{2} \times \ldots \times M^{s}
$$

e si noti che quest' ultima, come interferenza delle sottovarietà (2) di $V$, ha dimensione regolare:

$$
m^{1}+m^{2}+\ldots+m^{s}=m^{\prime 1}+m^{\prime 2}+\ldots+m^{s}-(s-1) v
$$

Con argomentazione analoga a quella accennata alla fine del n. 54, si prova inoltre che, nell'ipotesi che ciascuna delle $M^{i}$ sia effettiva e priva di componenti multiple, le (2) si segano semplicemente lungo la (3). Tenuto conto del n. 3 , ne consegue che è

$$
\left(M^{\prime 1} M^{\prime 2} \ldots M^{\prime s}\right)_{V}=M^{1} \times M^{2} \times \ldots \times M^{s} ;
$$

e la validità della (4) si estende subito al easo di varietà $M$ dotate di componenti multiple od anche virtuali, purchè in tali ipotesi si dia un'ovvia definizione per il secondo membro della (4). Ne discende che:

Quando le varietà (effettive o virtuali) $M^{1}, M^{2}, \ldots, M^{s}$ descrivono sulle $V^{\prime}$, $V^{2}, \therefore, V^{s}$ dei sistemi d'equivalenza, anche il prodotto diretto delle prime varia sul prodotto delle seconde entro ad un sistema d' equivalenza.

\section{§ II. - Riduzione del problema dell'intersezione con l'uso della varietà diagonale di nn prodotto.}

56. Approfondiamo ora lo studio della varietà di Segre $\left(2_{53}\right)$, nell'ipotesi ch'essa sia il prodotto di $s$ spazi aventi la stessa dimensione

$$
c_{\mathrm{i}}=c_{\mathrm{2}}=\ldots=c_{s}=c \text {. }
$$

In tal caso, questi spazi possono pensarsi come altrettante copie di un medesimo $S_{c}$, con l'intesa che punti $x^{1}, x^{2}, \ldots, x^{s}$ di $S_{c_{1}}^{4}, S_{c_{2}}^{2}, \ldots, S_{\mathcal{b}_{s}}^{s}$ imagini di uno stesso punto di $S_{r}$ abbiano le rispettive coordinate $\left(1_{53}\right)$ fra loro uguali (o proporzionali). Diremo che siffatti punti $x^{1}, x^{2}, \ldots, x^{s}$ sono fra loro coniu. gati. La varietà luogo dei punti $x=x^{1} \times x^{2} \times \ldots \times x^{s}$ di $T$ che corrispondono alle $\infty^{c} s$-ple di punti coniugati, chiamasi la varietà diagonale di $T$, e verrà denotata col simbolo $T^{\Delta}$.

Questa varietà si rappresenta con le $\left(3_{53}\right)$ in cui si faccia

$$
x_{j}^{4}=x_{j}^{2}=\ldots=x_{j}^{s} \quad(j=0,1, \ldots, c),
$$


ed è quindi una varietà di Veronese generalizzata, di dimensione $c$ ed ordine $s^{c}$, riferita birazionalmente ad $S_{c}$. Si ha inoltre che questa $T^{\Delta}$ appar. tiene ad un $S_{r}$ subordinato dello spazio $S_{d}$ di appartenenza di $T$, ove

$$
d=(c+1)^{s}-1, \quad \gamma==\left(\begin{array}{c}
c+s \\
s
\end{array}\right)-1 ;
$$

ed è subito visto che $T^{\Delta}$ è la completa interferenza di $T$ con $S_{Y}$, senza però generalmente risultare intersezione regolare di $T$ ed $S_{Y}$ in $S_{d}$, avendosi

$$
c s+\gamma-d<c \text { non appena sia } c>1, s \geq 2 \text { oppure } c=1, s>2
$$

(e cioè escluso soltanto il caso $c=1, s=2$ ). Proveremo tattavia che:

Preso un qualunque punto $x$ della varietà diagonale $T^{\Delta}$, si può determinare uno spazio $S_{a+c-c s}$ subordinato di $S_{a}$ (passante per $S_{\uparrow}$ ), per modo che - nell'intorno di $x$-tale $S_{d+c-c s}$ incontri T regolarmente e semplicemente lungo $T^{\perp}$.

Supposto, come non è restrittivo (n. 53), che il punto $x$ abbia diversa da zero la coordinata $x_{00 \ldots . .0}$, si potrà ammettere che in un intorno di $x$ valgano le $\left(4_{53}\right)$. Allora le $c(s-1)$ equazioni lineari indipendenti fornite dalle

$$
x_{j 00 \ldots 0}=x_{0 \ldots 0 j 0 \ldots 0},
$$

ove l'indice $j$ assuma $\mathrm{i}$ valori $1,2, \ldots, c$ ed a secondo membro si attribuisca ad esso uno qualunque degli $s-1$ posti che seguono il primo, rappresentano un $S_{d+c-s}$ subordinato di $S_{a}$. E subito visto che questo $S_{d+c-c s}$ nell' intorno di $x$ incontra $T$ precisamente lnngo $T^{\Delta}$; ed invero dalle (2), tenuto conto delle $\left(3_{\mathfrak{s} 3}\right),\left(4_{53}\right)$, seguono le (1). È poi chiaro che $T^{\Delta}$, come interferenza di $T$ ed $S_{d+c-c s}$ in $S_{d}$, ha dimensione regolare

$$
c s+(d+c-c s)-d=c .
$$

Inoltre $T^{\Delta}$ risulta intersezione semplice di $T$ ed $S_{d+c-c s}$, in quanto (n. 53) le cs coordinate $x_{j_{1} j_{2} \ldots j_{s}}$ che figurano nelle (2) sono parametri uniformizzanti relativamente all'intorno del punto $x$ su $T$.

57. Data una qualunque varietà algebrica irriducibile, di cui $V^{1}$ sia un modello proiettivo non singolare appartenente ad uno spazio lineare $S_{c}$ $\left(1 \leq v_{1}<c\right)$, si considerino $s(\geq 2)$ copie $S_{c}^{4}=S . . S_{c}^{2}, \ldots, S_{c}^{s}$ di questo spazio, ciascuna assieme con la relativa copia $V^{1}, V^{2}, \ldots, V^{s}$ di quella rarietà. Si potrà allora, giusta il n. 54, costruire la varietà

$$
V=V^{1} \times V^{2} \times . . \times V^{*}
$$

come varietì subordinata alla varietà di Segre

$$
T=S_{c}^{1} \times S_{c}^{2} \times \ldots \times S_{o}^{s} .
$$

La varietà $V$, cosl definita, si dirà ottenuta facendo il prodotto (diretto) della $V^{\prime} s$ volte per se stessa. 
Il luogo dei punti $x=x^{4} \times x^{2} \times \ldots \times x^{s}$ di $V$ che corrispondono alle $\infty^{v_{1}} s$-ple di punti coniugati di $V^{i}, V^{2}, \ldots, V^{s}$ (e cioè di punti imagini, sulle varie copie di $l^{\prime 1}$, di uno stesso punto di $\left.V^{i}\right)$, è una varietà algebrica birazionalmente identica a $V^{1}$ ed ha quindi la dimensione $v^{1}$. Questa varietà chiamasi la varietà diagonale $d i V$, e verrà denotata col simbolo $V^{\Delta}$.

Dalle precedenti definizioni delle $V, V^{\Delta}, T, T^{\Delta}$ si ha che le $\left(1_{56}\right)$ rappre. sentano tanto $T^{\perp}$ sopra $T$ quanto $V^{\lrcorner}$sopra $V$, sicchè risulta:

$$
V \Delta \doteq\left\langle V T^{\Delta}\right\rangle
$$

Va rilevato che, tuttavia, la $V \Delta$ non risulta mai intersezione regolare delle $V, T^{\Delta}$ in $T$ : ed invero, poichè attualmente si ha $v^{1}=v^{2}=\ldots=v^{s}$, le quattro varietà testè nominate hanno ora ordinatamente le dimensioni

$$
v^{1}, \quad s v^{1}, \quad c, s c \text {, }
$$

ed è $v^{1}+s v^{1}-s c<c$, essendo $s \geq 2, v^{1}<c$. Dimostreremo però che :

$L a$ varietà $V^{\Delta}$ risulta priva di punti multipli, ed è intersezione semplice (non regolare) di $V$ e $T^{\Delta} s u T$. Pì precisamente, denotando con $x$ un qualunque punto di $V^{\Delta}$, la varietà $V^{\Delta}$ - in un intorno di $x$ - può ottenersi come intersezione semplice e regolare $d i V$ con un opportuno spazio $S_{d+v^{1}-s v^{1}}$ giacente nell' $S_{d} d i$ appartenenza di $T$ e passante per lo spazio $S_{Y}$ di appartenenza di $T^{4}$.

Il punto $x^{1}$ di $V^{\prime}$, che corrisponde al punto $x$ suddetto di $V^{\Delta}$, ̀̀ punto semplice di $V^{\prime}$; si potranno quindi scegliere in $S_{c}^{1}$ coordinate $x_{0}^{1}, x_{1}^{1}, \ldots, x_{c}^{1}$ tali che - in quel punto $x^{1}-$ e quindi pure in un intorno di esso in $S_{c}^{1}-$ sia $x_{0}^{1}=1$, e le $x_{1}^{1}, x_{z}^{1}, \ldots, x_{v^{1}}^{1}$ risultino inoltre parametri uniformizzanti in un intorno di $x^{1}$ su $V^{1}$. Allora negli intorni dei punti $x^{4}, x^{z}, \ldots, x^{s}$ coniugati ad $x^{1}$ in $S_{c}^{1}, S_{c}^{2}, \ldots, S_{c}^{s}$, ed in un intorno di $x$ in $S_{d}$, potranno supporsi valide le $\left(4_{53}\right)$.

Consideriamo le equazioni

$$
x_{j 00 \ldots 0}=x_{0 \ldots 0 j 0 \ldots 0},
$$

ove l'indice $j$ assuma $\mathrm{i}$ valori $1,2, \ldots, v^{1}$ ed a secondo membro si attribuisca ad esso uno qualnnque degli $s-1$ posti che seguono il primo. Si hanno così in tutto $v^{1}(s-1)$ equazioni lineari indipendenti, rappresentanti un $S_{d+v^{1-s x^{2}}}$ di $S_{d}$. Da esse, tenuto conto delle $\left(3_{53}\right),\left(4_{53}\right)$, si traggono subito le

$$
x_{j}^{1}=x_{j}^{2}=\ldots=x_{j}^{8} \quad \text { per } j=0,1, \ldots, v^{1} ;
$$

e queste relazioni valgono pure per $j=v^{4}+1, v^{1}+2, \ldots, c$ negli intorni di $x^{4}, x^{2}, \ldots, x^{s}$ su $V^{1}, V^{2}, \ldots, V^{s}$, in conseguenza delle equazioni di tali intorni. Poichè - in un intorno di $x$ su $V$ - valgono queste ultime equazioni e le $\left(3_{53}\right)$, mentre d'altro canto le $\left(1_{56}\right)$ sono le equazioni di $V^{\Delta}$ su $V$, così - nel suddetto intorno - VA risulta l'intersezione di $V$ con lo spazio rappresentato dalle (3). 
Tale intersezione ̀̀ regolare, essendo

$$
v^{1}=s v^{1}+\left(d+v^{1}-s v^{1}\right)-d
$$

essa è inoltre semplice, onde il punto $x$ è semplice per $V \Delta$, in quanto le $s v^{\prime}$ coordinate $x_{j_{1} j_{2} \ldots j_{s}}$ che figurano nelle (3), essendo rispettivamente uguali ai parametri $x_{j}^{i}$ che compaiono nelle (4), risultano parametri uniformizzanti relativamente all' intorno del panto $x$ su $V$.

Osserviamo da ultimo che, in virtù del n. 9 e del precedente teorema, la (2) forniso l' equivalenza

$$
\left(V T^{\Delta}\right)_{T}^{V^{\Delta}}=0
$$

la quale risulta non banale non appena si supponga $v^{1} \geq\left(1-\frac{1}{s}\right) c$.

58. Siano $M^{1}, M^{2}, \ldots, M^{s} s$ varietà di $V^{t}$ incontrantisi regolarmente $\theta$ semplicemente lungo una varietà pura, $Q^{1}$, di dimensione

$$
q=m^{1}+m^{2}+\ldots+m^{s}-(s-1) v^{1} \geq 0 .
$$

Prendiamo $s$ copie $V^{1}, V^{2}, \ldots, V^{s}$ distinte di $V^{1}$ e, per non moltiplicare inutilmente le notazioni, denotiamo ancora con $M^{1}, M^{2}, \ldots, M^{s}$ le copie delle $M^{1}, M^{2}, \ldots, M^{s}$ ordinatamente sulle $V^{1}, V^{2}, \ldots, V^{s}$. Costruiamo, come nel n. 57, la varietà $V$ prodotto della $V^{1} s$ volte per se stessa e, entro questa, consideriamo il prodotto diretto

$$
M=M^{1} \times M^{2} \times \ldots \times M^{s} .
$$

Sulla $V$, vi è inoltre luogo a considerare la varietà diagonale $V \Delta$, birazionalmente identica a $V^{1}$ (n. 57); e sia $Q$ la varietà (di dimensione $q$ ) che corrisponde a $Q^{i}$ nel riferimento birazionale senza eccezioni fra $V^{1}$ e $V^{\Delta}$. Ci proponiamo di dimostrare che:

La varietà $Q$ risulla l'intersezione, regolare e semplice, delle varietà $M$ e VA entro $V$.

E anzitutto chiaro, dalle precedenti definizioni, che $Q$ è l'interferenza delle $M, V \Delta$, e cioè :

$$
Q \doteq\left(M V^{\Delta}\right)
$$

Poichè la dimensione $q$ di $Q$ si esprime con la (1), quella di $M$ - a norma della (1), e tenuto conto del n. 54 - vale $m^{i}+m^{2}+\ldots+m^{s}$, mentre $V^{\Delta}$ e $V$ hanno le dimensioni $v^{1}$ ed $s v^{1}$, cosi $Q$ e intersezione regolare di $M$ e $V^{\Delta}$ in $V$.

Sia $x^{1}$ un qualunque punto semplice di $V^{1}$, semplice per $Q^{1}$ e per ciascuna delle $M^{i}$, nel quale le $M^{1}, M^{2}, \ldots, M^{s}$ s'incontrino semplicemente (il che significa che gli spazi tangenti in $x^{1}$ alle $M^{4}, M^{2}, \ldots, M^{s}$ hanno per intersezione lo spazio tangente in $x^{1}$ a $\left.Q^{1}\right)$. Detti $x^{2}, \ldots, x^{s}$ i punti coniugati di $x^{1}$ sulle $V^{2}, \ldots, V^{s}$, i quali apparterranno quindi ordinatamente alle $M^{2}, \ldots, M^{s}$, si potranno allora, negli spazi $S_{c}^{i}$ di appartenenza delle $M^{i}(i=1,2, \ldots, s)$, 
introdurre coordinate $x_{0}^{i}, x_{1}^{i}, \ldots, x_{c}^{i}$ tali che, in un intorno del punto $x^{t}$, si abbia $x_{0}^{i}=1$ ed ivi inoltre le

$$
\begin{aligned}
& x_{1}^{i}, x_{2}^{i}, \ldots, x_{v}^{i}, \\
& x_{1}^{i} \ldots x_{4}^{i} x_{m}^{i}+\ldots+m^{i-1}-(i-2) q+1 \ldots x_{m}^{i}+\ldots+m^{i}-(i-1) q
\end{aligned}
$$

risultino parametri uniformizzanti ordinatamente su $V^{i}, M^{i}$. Ne consegue che nel punto $x=x^{i} \times x^{2} \times \ldots \times x^{s}$ può assumersi $x_{00 \ldots 0}=1$, ed inoltre - in un intorno di questo punto sulle varietè $V, M, V^{\Delta}, Q$ - possono fungere rispet. tivamente da parametri uniformizzanti :

le $c v^{4}$ coordinate $x_{0 . . .0 j 0 \ldots 0}$ $\left(j=1,2, \ldots, v^{1}\right)$

le $m^{1}+m^{2}+\ldots+m^{s}$ coordinate $x_{0 \ldots 0 j s . .0}$, ove l'indice $j$ sia all' $i$-mo posto $(i=1,2, \ldots, s)$ ed assuma gli $m^{i}$ valori $1,2, \ldots, q, m^{1}+\ldots+m^{i-1}-$ $-(i-2) q+1, \ldots, m^{1}+\ldots+m^{4}-(i-1) q$

le $v^{1}$ coordinate $x_{0 . \ldots 0}, x_{20} \ldots 0, \ldots, x_{v_{1} 0 \ldots 0}$

le $q$ coordinate $x_{10 \ldots 0}, x_{20 \ldots 0}, \ldots, x_{q 0 \ldots 0}$.

Cio implica che, entro lo spazio tangente nel punto $x$ a $V$, gli spazi ivi tangenti alle $M, V^{\Delta}$ s'incontrino regolarmente, secondo lo spazio tangente in $x$ a $Q$; e cio completa la dimostrazione dell'asserto.

Dalla proposizione testè stabilita, scrivendo $M$ in luogo di $M^{1}$ ed assumendo in particolare $M^{z}=V^{2}, \ldots, M^{s}=V^{s}$, si ricava che :

Nel riferimento birazionale che intercede fra una data varietà $V^{1}$ e la varietà diagonale $V^{\Delta}$ della varietà $V$, prodotto della $V^{1} s$ volte per se stessa, ad una qualunque sottovarietà $M$ di $V^{4}$ corrisponde la varietà $M^{\Delta}=\left(M^{\prime} V \Delta\right)_{V}$, ove $\boldsymbol{M}^{\prime}=M \times V^{2} \times \ldots \times V^{s}$.

59. Con argomentazioni del tutto analoghe a quelle del numero precedente, si vede che, più generalmente, se le varietà $M^{1}, M^{2}, \ldots, M^{s}$ dì $V^{1}$ s'incontrano semplicemente lango due varieta pure $Q^{\prime}$ e $P^{1}$, la prima di dimensione regolare $q$ e la seconda di dimensione $p(\geq q)$, allora

Le varietà $M$ e $V^{\Delta}$ s'incontrano semplicemente entro $V$ lungo le varietà $Q$ e $P$ imagini delle $Q^{1}$ e $P^{1}$ nel riferimento birazionale fra $V^{1}$ e $V^{\Delta}$.

Questo risultato puo naturalmente venire sfruttato anche localmente, il che permette di ricondarre lo studio del comportamento delle $M^{4}, M^{2}, \ldots, M^{s}$ nell'intorno di un punto $x^{1}$ ad esse comune, allo studio del comportamento delle $M, V^{\Delta}$ nell'intorno del punto omologo $x=x^{1} \times x^{2} \times \ldots \times x^{s}$. Ma allora, per quest' ultimo studio, la $V^{\Delta}$ può venir sostituita con uno spazio lineare $S_{d+v^{1}-s v^{1}}$, in virtù del a. 57 . L' uso delle varietà prodotto nei problemi d'intersezione entro una data varietà ambiente non singolare $\left({ }^{20}\right)$, offre quindi il

$\left.{ }^{20}\right)$ Esso trovasi già in B. SEGRE [5], o prima ancora parzialmente in Zeuthen, C. Segre, Severi, e fu quindi sfruttato in modo sistematico da WEIL [1] e da altri Autori; efr. spe. cialmente Severi [11]. 
vantaggio di sostituire ad nn qualunque numero $s$ di varietà da intersecare due sole varietà, una delle quali è anzi fissa e non singolare, e puo addirittura supporsi essere uno spazio lineare quando ci si limiti a questioni di carattere locale.

Aggiungasi che, tenuto conto dei nn. 3, 9, 55, e con le notazioni precedenti, da quanto sopra si trae che

$$
\begin{aligned}
& \text { la } Q^{1}=\left(M^{1} M^{z} \ldots M^{s}\right)_{V^{1}} \text { equivale alla } Q=\left(M V^{4}\right)_{V}, \\
& \text { la } Q^{1}=\left(M^{1} M^{2} \ldots M^{s}\right)_{V^{1}}^{\text {equivale alla } Q} Q=\left(M V^{\Delta}\right)_{V}^{P} .
\end{aligned}
$$

Così, ad esempio, la $\left(5_{57}\right)$ appare in tal guisa una conseguenza dell'ovvia relazione $\left(V^{1} V^{1} \ldots V^{1}\right)_{S_{c}^{1}}^{V^{1}}=0$.

\section{\$ III. - Successioni ed intersezioni di sottovarietà prodotti entro una varietà prodotto.}

60. Date $s(\geq 2)$ varietà irriducibili e non singolari $V^{2}, V^{2}, \ldots, V^{s}$, si considerino sulla $V^{i}$ due varietà pure $M^{i}, N^{i}$ qualsiansi, e si ponga:

$$
P^{i}=\left(M^{i} N^{i}\right)_{V i} \quad(i=1,2, \ldots, s) .
$$

Allora, a norma del n. 55, sulla varietà

$$
V=V^{2} \times V^{2} \times \ldots \times V^{s}
$$

restano definite le

$$
\begin{gathered}
M=M^{1} \times M^{2} \times \ldots \times M^{s}, \quad N=N^{1} \times N^{2} \times \ldots \times N^{s}, \\
P=P^{1} \times P^{2} \times \ldots \times P^{s} .
\end{gathered}
$$

Vogliamo dimostrare che

Dalle (1), (2), (3) segue la

$$
P=(M N)_{V} \text {. }
$$

A tal uopo, consideriamo su $V$ le varietà $M^{\prime i}$ determinate in funzione delle $M^{i}$ mediante le $\left(1_{55}\right)$, e le varieta $N^{\prime i}, P^{\prime i}$ similmente definite dalle $N^{i}, P^{i}$. Incominciamo col provare che

In forza della (1), vale l' equivalenza

$$
P^{\prime i}=\left(M^{\prime i} N^{\prime i}\right)_{V} \text {. }
$$

Basterà stabilire questo risultato nell'ipotesi in cui $M^{i}$ ed $N^{i}$ siano varietà effettive di $V^{i}$ segantisi regolarmente e semplicemente lungo la $P^{i}$, poichè da qui si deduce il risultato nel caso generale per somma e sottrazione, usufruendo della proposizione finale del n. 55. Ora, nell'ipotesi suddetta, si ha manifestamente

$$
P^{\prime i} \doteq\left\langle M^{\prime i} N^{\prime i}\right\rangle
$$

e le $M^{i}, N^{i}$ s'incontrano regolarmente in $V$ lungo $P$, poichè, posto

$$
k=v^{1}+\ldots+v^{i-1}+v^{i+1}+\ldots+v^{s}
$$


risulta $m^{\prime i}=m^{i}+k, \quad n^{\prime i}=n^{i}+k, p^{i}=p^{i}+k, \quad v=v^{i}+k$, sicchè dalla $p^{i}=m^{i}+n^{i}-v^{i}$ segue la $p^{i}=m^{i}+n^{i}-v$. Con argomentazioni analoghe a quelle svolte in casi consimili nel paragrafo precedente, si vede poi subito che $M^{\prime i}$ ed $N^{\prime i}$ si segano semplicemente lungo $P^{\prime i}$. Dalla (6) segue allora senz' altro la (5).

Cio premesso, basta applicare ripetutamente la $\left(4_{55}\right)$, assieme alle (1), (2), (3), (5), per vedere che dे

$$
\begin{aligned}
P & =P^{1} \times P^{2} \times \ldots \times P^{s}=\left(P^{\prime \prime} P^{\prime 2} \ldots P^{\prime s}\right)_{V}= \\
& =\left(\left(M^{\prime \prime} N^{\prime \prime}\right)_{V}\left(M^{\prime 2} N^{\prime 2}\right)_{V} \ldots\left(M^{\prime s} N^{\prime s}\right)_{V}\right)_{V}= \\
& =\left(\left(M^{\prime 1} M^{\prime 2} \ldots M^{\prime s}\right)_{V} \cdot\left(N^{\prime 1} N^{\prime 2} \ldots N^{\prime s}\right)_{V}\right)_{V}= \\
& =\left(\left(M^{1} \times M^{2} \times \ldots \times M^{s}\right) \cdot\left(N^{1} \times N^{2} \times \ldots \times N^{s}\right)_{V}=(M N)_{V},\right.
\end{aligned}
$$

donde la (4).

Il risultato testè ottenuto si estende immediatamente al caso in cui sulle $V^{i}$ si consideri uuo stesso numero, anche maggiore di due, di varietà $M^{i}, N^{i}, \ldots, R^{i}$. Si perviene cosi all' equivalenza;

$$
\begin{gathered}
\left(M^{1} N^{1} \ldots R^{1}\right)_{V^{1}} \times\left(M^{2} N^{2} \ldots R^{2}\right)_{V^{2}} \times \ldots \times\left(M^{s} N^{s} \ldots R^{s}\right)_{V^{s}}= \\
=\left(\left(M^{1} \times M^{2} \times \ldots \times M^{s}\right) \cdot\left(N^{1} \times N^{2} \times \ldots \times N^{s}\right) \ldots\left(R^{1} \times R^{z} \times \ldots \times R^{s}\right)_{V} .\right.
\end{gathered}
$$

61. Data sopra ciascuna delle $V^{i}$ una successione

$$
\left|V^{i}\right|=V_{0}^{i}, V_{1}^{i}, V_{2}^{i}, \ldots,
$$

di sostegno $V^{i}$, possiamo considerare sul prodotto $V=V^{4} \times V^{*} \times \ldots \times V^{s}$ dei relativi sostegni le varietà pure, di dimensione $v-j$, definite dalle formule

$$
V_{j}=\Sigma V_{h_{1}}^{\mathrm{l}} \times V_{h_{2}}^{\mathrm{s}} \times \ldots \times V_{h_{3}}^{\mathrm{s}} \quad(j=0,1,2, \ldots),
$$

dove - per $j$ fissato - la somma sia estesa a tutte le soluzioni in interi $h$ delle

(3) $j=h_{1}+h_{2}+\ldots+h_{s}, \quad h_{1} \geq 0, h_{2} \geq 0, \ldots, h_{s} \geq 0$.

E subito visto che le (2) costituiscono alla loro volta una successione $: \nabla !$, di sostegno $V$; chiameremo questa il prodotto diretto delle (1), e scriveremo che è :

$$
\left.|V|=\left|V^{1} i \times\right| V^{2}|\times \ldots \times| V^{s}\right\} .
$$

Semplificheremo questa scrittura ponendo in essa $V^{i}$ in luogo di $\left|V^{i}\right|$, nel caso particolare in cui la successione (1) si riduca alla

$$
\left.\mid \nabla^{i}\right\}=\nabla^{i}, 0,0, \ldots \text {; }
$$

questa notazione $\mathrm{e}$ in accordo col fatto che, allora, nella somma (2) basta limitarsi ai termini provenienti dal valore zero dell'indice $h_{i}$, sicchè in ogni termine di tale somma compare $V^{i}$ come $i$-mo fattore. 
Dalla (2), avato riguardo alla (3) ed al n. 6, segue subito che:

L'alternante del prodotto diretto di due o più successioni, non è altro che il prodotto diretto delle successioni alternanti di queste ultime.

Si considerino ora su ciascuna delle suddette varieta $V^{i}$ due sucoes. sioni $\left|V^{\prime} i\right|,\left|V^{\prime i}\right|$ che l'ammettano come sostegno, e si ponga

$$
\left|V^{i}\right|=\left(\left|V^{i}\right| \cdot \mid V^{\prime \prime} i\right)_{V^{i}} \quad(i=1,2, \ldots, s) .
$$

Ci proponiamo di mostrare che

Definite le $\left.|V|, \mid V^{\prime}\right\},\left|V^{\prime \prime}\right|$ con la $(3)$ e con le analoghe relazioni

$$
\left.\left|V^{\prime} i=\right| V^{\prime 1}|\times| \nabla^{\prime 2}|\times \ldots \times| V^{\prime s}|, \quad| \nabla^{\prime \prime}|=| V^{\prime 1}|\times| V^{\prime \prime 2}|\times \ldots \times| \nabla^{\prime \prime}\right\},
$$

in forza delle (5) risulta:

$$
|v|=\left(\left|V^{\prime}\right| \cdot \mid \nabla^{\prime \prime}\right)_{v}
$$

Dalle (2), (5) si trae infatti che è:

$$
\nabla_{j}=\Sigma\left(V_{h^{\prime}}^{\prime 1}, V_{h^{\prime \prime}}^{\prime \prime 1}\right)_{V^{1}} \times\left(V_{h^{\prime}}^{\prime \prime}, V_{h^{\prime \prime 2}}^{\prime 2}\right)_{V^{2}} \times \ldots \times\left(V_{h_{s}^{\prime}}^{\prime s}, V_{h_{s}^{\prime \prime}}^{\prime \prime s}\right)_{V^{\prime \prime}}
$$

dove la somma dev'essere estesa a tutte le $h \geq 0$ per cui siano soddisfatte le $(3)$ e le

$$
h_{1}^{\prime}+h^{\prime \prime}{ }_{1}=h_{1}, \quad h_{2}^{\prime}+h^{\prime \prime}=h_{2}, \ldots, h_{s}^{\prime}+h^{\prime \prime}{ }_{k}=h_{s} .
$$

In virtù del n. 60 , ne consegue:

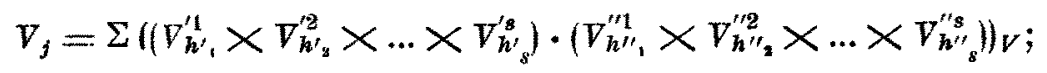

e si osservi che gli indici in quest' ultima somma debbono assumere tatti e soli $i$ valori interi $h \geq 0$ soddisfacenti alle

$$
h_{1}^{\prime}+h_{2}^{\prime}+\ldots+h_{s}^{\prime}=k, \quad h^{\prime \prime}{ }_{1}+h^{\prime \prime}{ }_{2}+\ldots+h^{\prime \prime}{ }_{s}=j-k,
$$

ove a $k$ si diano $i$ valori $0,1, \ldots, j$. Avuto riguardo al $n .7$ ed alle precedenti definizioni, si ha quindi la (6).

Assunto in particolare $\left.\left|\nabla^{\prime \prime i}\right|=\mid \tilde{V}^{i i}\right\}$, le (5), (4) forniscono le

$$
\left|\nabla^{i}\right|=\nabla^{i}, 0,0, \ldots, \quad|\nabla|=\nabla, 0,0, \ldots .
$$

Il precedente teorema mostra allora che:

L'inversa della successione prodotto diretto di due o più successioni, coincide col prodotto diretto delle inverse di queste ultime.

Aggiungasi che il suddetto teorema si estende subito induttivamente al caso in cui, su ciascuna delle $V^{i}$, si consideri - in un certo ordine - uno stesso numero $(\geq 2)$ arbitrario di successioni; sicche: il prodotto diretto delle intersezioni di queste, uguaglia l'intersezione dei prodotti diretti delle successioni aventi in quell' ordine uno stesso posto. 
62. Date due varieta $\nabla, W$ non singolari, consideriamone il prodotto

$$
V^{\prime}=\nabla \times W
$$

allora, detta $M$ una qualunque sottovarieta di $V$, denotiamo con $M^{\prime}$ la varieta di $\nabla^{\prime}$ definita dalla

$$
M^{\prime}=M \times W .
$$

Con tali notazioni, la prima proposizione del n. 60 fornisce in particolare l'uguaglianza

$$
\left(M^{1} M^{2} \ldots M^{s}\right)_{V}^{\prime}=\left(M^{1 \prime} M^{2 \prime} \ldots M^{s^{\prime}}\right)_{V^{\prime}}
$$

Più generalmente, avuto riguardo alla definizione del n. 9, si vede senza difficoltà che è

$$
\left[\left(M^{1} M^{2} \ldots M^{s}\right)_{V}^{P}\right]^{\prime}=\left(M^{1^{\prime}} M^{*^{\prime}} \ldots M^{s^{\prime}}\right)_{V^{\prime}}^{P^{\prime}} .
$$

Cio premesso, rifacciamoci alle considerazioni del n. 13 relative alle varietà $P, A^{1}, A^{2}, \ldots, A^{s}$ di $\nabla$, ed osserviamo che considerazioni analoghe possono svolgersi per le corrispondenti varietà $P^{\prime}, A^{t^{\prime}}, A^{2^{\prime}}, \ldots, A^{s^{\prime}}$ di $\nabla^{\prime}$. Più precisamente, poíchè $v^{\prime}=v+w, p^{\prime}=p+w$, se valgono le $\left(1_{13}^{\prime},\left(2_{13}\right)\right.$ si ha conseguentemente

$$
v^{\prime}-p^{\prime} \leq s \leq v^{\prime}-w, \quad t=s+p^{\prime}-v^{\prime} \quad\left(0 \leq t \leq p \text { o } p^{\prime}-w\right) ;
$$

inoltre le $A^{\prime}$ sono ipersuperficie di $V^{\prime}$ per le quali, con le notazioni del n. 13 , risulta :

$$
\nabla_{i}^{\prime}\left(A^{\prime}\right)=\left[\nabla_{i}^{s}(A)\right]^{\prime} .
$$

Pertanto, uguagliando i prodotti diretti per $W$ dei due membri della $\left(4_{13}\right)$ ed applicando il primo capoverso del presente numero, otteniamo

$$
\left(A^{1^{\prime}} A^{2^{\prime}} \ldots A^{s^{\prime}}\right)_{V^{\prime}}-\left(A^{1^{\prime}} A^{2^{\prime}} \ldots A^{s^{\prime}}\right)_{V^{\prime}}^{p^{\prime}}=\sum_{i=0}^{t}\left(P_{V, i}\right)^{\prime} \nabla_{t-i}^{s^{s}}\left(A^{\prime}\right) \text {. }
$$

Basta ora confrontare le relazioni che così si hanno per $t=0,1, \ldots, p$ (e cioè per. $s=v-p, v-p+1, \ldots, v)$, ordinatamente con le relazioni

$$
\left.\left(A^{1 \prime} A^{z^{\prime}} \ldots A^{s^{\prime}}\right)_{V^{\prime}}-\left(A^{1^{\prime}} A^{z^{\prime}} \ldots A^{s^{\prime}}\right)_{V^{\prime}}^{P^{\prime}}=\sum_{i=0}^{t} P^{\prime} V^{\prime}, i \nabla_{t-i}^{\prime s} A^{\prime}\right)
$$

del pari fornite dal n. 13, per dedurre le

$$
P^{\prime}{ }^{\prime}, i=\left(P_{V, i}\right)^{\prime} \quad \text { per } i=0,1, \ldots, p .
$$

Rileviamo infine che la (1) sussiste anche per $s=v+1, v+2, \ldots, v^{\prime}$ (e quindi per $t=p+1, p+2, \ldots, p^{\prime}$ ), purchè le $A^{\prime}$ stiano ivi per indicare $s$ ipersuperficie di $V^{\prime}$ passanti genericamente per $P^{\prime}$; e, come tali, possono assumersi le $A^{i} \times W$, dove le $A^{i}$ siano $s$ ipersuperficie di $\nabla$ passanti generi. camente per $P$. Corrispondentemente risulta

$$
\left(A^{y^{\prime \prime}} A^{z^{\prime}} \ldots A^{s^{\prime}}\right)_{V^{\prime}}=0, \quad\left(A^{\prime \prime} A^{2 \prime} \ldots A^{z^{\prime}}\right)_{V^{\prime}}^{\mathrm{P}^{\prime}}=0,
$$


sicchè le $w$ equazioni date dalla (1) per i suddetti valori di $s$ e $t$ forniscono le:

$$
\sum_{i=0}^{p+h} P_{V}^{\prime}, i \nabla_{p+h-i}^{\prime 2+h}\left(A^{\prime}\right)=0 \quad \text { per } h=1,2, \ldots, w .
$$

Ora ciascuna delle varietà $\left(P_{V, i} \nabla_{p+h-i}^{v+h}(A)\right)_{V}$ ha dimensione virtuale $-h<0$, ed $\theta$ quindi nulla. In virtù del primo capoverso del presente numero e delle (2), $i$ singoli termini delle somme a primo membro nelle (3) risultano nulli per $i=0,1, \ldots, p$. Le (3) riduconsi pertanto alle

$$
{\underset{i=p+1}{p+h} P_{V}^{\prime}, i}_{p+h-i}^{\prime v+h}\left(A^{\prime}\right)=0 \quad \text { per } h=1,2, \ldots, w
$$

le quali successivamente porgono le

$$
P^{\prime} v^{\prime}, i=0 \quad \text { per } i=p+1, p+2, \ldots, p^{\prime} .
$$

Queste mostrano che le (2) sussistono anche per tutti i valori di $i$ maggiori di $p$, onde le (2), (4) si compendiano con la

$$
\left|P^{\prime}{ }^{\prime}\right|=\left|P_{V}\right|^{\prime}
$$

ossia, sotto forma più esplicita, con la

$$
\left|(P \times W)_{V \times W}\right|=\left|P_{V}\right| \times W .
$$

63. Dimostreremo ora che

Date $s(\geq 2)$ varietà irriducibili e non singolari $V^{i}$, e scelta comunque ordinatamente in ciascuna di esse una varietà irriducibile e non singolare $M^{i}(i=1,2, \ldots, s)$, il prodotto $M=M^{1} \times M^{2} \times \ldots \times M^{s}$ è una sottovarietà della $V=V^{1} \times \nabla^{2} \times \ldots \times V^{s}$, per la quale risulta

$$
\left.\left|\boldsymbol{M}_{V}\right|=\left|\boldsymbol{M}_{V^{1}}^{1}\right| \times\left|\boldsymbol{M}_{V^{2}}^{2}\right| \times \ldots \times \mid \boldsymbol{M}_{V}^{s}\right\} \text {. }
$$

A tal uopo, consideriamo su $V$ le varietà $M^{\prime} i$ definite dalle $\left(1_{55}\right)$, e rammentiamo che vale la $\left(4_{55}\right)$. In forza del teorema del prodotto (n. 20), abbiamo quindi :

$$
\left|M_{V}\right|=\left|\left(M^{1} \times M^{2} \times \ldots \times M^{s}\right)_{V}\right|=\left(\left|M_{V}^{\prime}\right| \cdot\left|M_{V}^{\prime 2}\right| \ldots\left|M_{V}^{\prime s}\right|_{V} .\right.
$$

D' altra parte, avuto riguardo alle $\left(1_{55}\right)$, e tenuto conto della $\left(5_{62}\right)$, si ha:

$$
\left.M_{V}^{i}\right\}=V^{i} \times \ldots \times V^{i-1} \times\left|M_{V i}^{i}\right| \times V^{i+1} \times \ldots \times V^{s} .
$$

Basta dunque sostituire nella (2) queste espressioni in luogo delle $\mid M_{V}^{\prime i}$, applicare il n. 61 ed osservare che è

$$
\left(\mid M_{V i}^{i} ! V^{i} V^{i} \ldots V^{i}\right)_{V i}=\mid M_{V i}^{i},
$$

per dedurre la (1). 


\section{CAPITOLO SESTO}

\section{LE SUCCESSIONI CANONICHE SULLE VARIETA ALGEBRICHE}

\section{\&. - Successioni invarianti di dato sostegno.}

64. Data una qualunque varieta algebrica $V^{i}$, irriducibile e non singolare, si consideri il prodotto di $V^{\mathrm{i}}$ per se stessa:

$$
V=V^{1} \times V^{2}
$$

(dove $\nabla^{2}$ denoti una copia di $V^{1}$ ), e - su questo - la varietà diagonale, $V^{12}$ (n. 57). È chiaro che, se si sostituisce a $V^{1}$ una varieta $\bar{V}^{1}$ ad essa riferita in una corrispondenza birazionale $\theta$ che sia regolare (e cioè senza eccezioni), anche la (1) e l'analoga varietà

$$
\bar{V}=\overline{V^{1}} \times \overline{V^{2}}
$$

vengono a corrispondersi in una trasformazione regolare, $T$; e questa muta la $V^{18}$ nella varietà diagonale $\overline{V^{12}}$ di $\bar{V}$. In virtù del $\S \mathrm{I}$ del Cap. $2^{\circ}$, la $T$ muta conseguentemente la successione covariante $d^{\prime}$ immersione $\left|V_{V}^{i 2}\right|$ nella $\left\{\overline{\nabla_{\bar{V}}^{12}}\right\}$.

Rileviamo ora che la corrispondenza subordinata da $T$ fra $V^{12}$ e $\overline{V^{11}}$ non è che il prodotto $\Omega \Theta \overline{\mathbf{\Omega}}^{-1}$, dove $\Omega, \bar{\Omega}$ denotino le trasformazioni birazionali senza eccezioni intercedenti - giusta il n. 57 - fra le $V^{12}, \overline{V^{12}}$ e le $V^{\prime}, \overline{V^{1}}$. Ne consegue che le $\boldsymbol{\Omega}, \overline{\boldsymbol{\alpha}}$ debbono trasformare le $\left\{V_{V}^{12}\right\},\left\{\overline{V_{\bar{V}}^{12}}\right\}$ in due successioni $\left.\left\{V^{i}\right\}, \mid \overline{V^{i}}\right\}$, rispettivamente di sostegni $V^{i} \Theta \overline{V^{l}}$, le quali risultano mutate l'una nell'altra dalla trasformazione birazionale $\Theta$ fra $V^{1}$ e $V^{1}$. Pertanto:

La successione $\left.\mid V^{1}\right\}$, dianzi costruita su $V^{1}$ come trasformata di $\left|V_{V}^{12}\right|$ mediante $\Omega$, risulta invariante di fronte alle trasformazioni birazionali regolari di $\nabla^{1}$.

65. Si possono subito ottenere su $V^{\prime}$ altre successioni covarianti, operando in uno dei due modi seguenti.

Un primo modo $\theta$ quello di considerare un qualunque numero $s(\geq 2)$ di copie $V^{1}, V^{2}, \ldots, V^{s}$ di $V^{1}$, e di riferirsi alla varieta diagonale $V^{\Delta}$ del prodotto diretto

$$
V=V^{2} \times V^{2} \times \ldots \times V^{s}
$$

Fra $V^{\Delta}$ e $V^{1}$ intercede una trasformazione birazionale regolare, $\Omega$, la quale muta la successione covariante d'immersione $\left|V_{V}^{\Delta}\right|$ in una suceessione invariante di sostegno. $V^{\perp}$, che denoteremo con $\left\{V_{|s|}^{1}\right\}$. Ciò si vede mediante considerazioni perfettamente analoghe a quelle del n. 64 ; ed o chiaro che, con le notazioni ivi introdotte, risulta

$$
\left\{V_{|2|}^{*}\right\}=\left\{V^{1}\right\} .
$$

Annali di Matematica 
Un secondo modo si ottiene moltiplicando fra loro in $\mathfrak{A}_{V^{1}}$ le successioni dianzi ottenute, il che conduce - fra l'altro - alle successioni invarianti

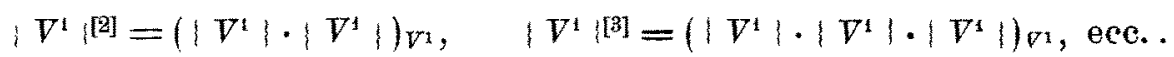

Dimostreremo che :

Le successioni invarianti suddette sono legate fra loro ed alla $\left\{V^{1}\right\}$ del n. 64 in modo estremamente semplice, risultando precisamente

$$
\left\{\nabla_{\{s\}}^{1}\right\}=\left\{V ^ { 1 } \left\{^{[s-1]}\right.\right. \text {. }
$$

Poichè se $s=2$ la (3) si riduce alla (2), basterà stabilire la (3) nell'ipotesi che sia $s \geq 3$. A tal uopo, denotando come dianzi con $V^{12}$ la varietà diagonale di $V^{1} \times V^{2}$, poniamo

$$
\overline{V^{12}}=V^{12} \times V^{3} \times \ldots \times V^{8},
$$

ed introduciamo dei simboli $V^{14}, \overline{V^{14}}$ aventi (per $i=3, \ldots, s$ ) significati analoghi a quelli testè specificati per $i=2$. Fra $V^{1 t}$ e $V^{t}$ intercede una trasformazione birazionale regolare, che denoteremo con $\Omega^{(i)}$, la quale - giusta il n. 64 - muta la successione $\left|V_{V^{i} \times V^{i}}^{1 i}\right|$ nella $\left\{V^{\prime}\right\}$.

Avendo dianzi indicato con $\Omega$ la corrispondenza i.irazionale $V^{\Delta}$ e $V^{1}$, ne consegue che fra $V^{i}$. e $V^{\Delta}$ intercede la corrispondenza birazionale $\Omega^{(i)} \mathbf{Q}^{-1}$. E si vede facilmente, riferendoci, per semplicità di scrittura, al caso $i=2$, che l'intersezione - entro $V-$ di $V^{\Delta}$ con la successione

$$
\left\{V_{V^{1} \times V^{2}}^{12}\right\} \times V^{3} \times \ldots \times V^{s}
$$

non è altro che la trisformata della $\left\{\nabla_{V^{1} \times V^{2}}^{12}\right\}$ mediante la $Q^{(2)} \Omega^{-1}$. Tale intersezione, che denoteremo con $\left\{\nabla_{\mid 2\}}^{\Delta}\right\}$, viene dunque trasformata da $\mathbf{\Omega}$ nella $\left\{V^{1}\right\}$; e si può qui - con ovvio significato dei simboli - invece dell'indice 2 porre uno qualunque dei numeri $3, \ldots, s$.

Ciò premesso, in virtù della definizione delle varietà diagonali (n. 56) e mediante argomentazioni consimili a quelle svolte nel § II del Cap. 5o, si ottiene l'equivalenza :

$$
V^{\Delta}=\left(\overline{V^{12}} \overline{\nabla^{13}} \ldots \overline{V^{1 s}}\right)_{V}
$$

Da qui, in forza del teorema del prodotto (n. 20), si trae la

$$
\left.\left.\left\{\nabla_{V}^{\Delta}\right\}=\left(\left|\overline{V_{V}^{1 \overline{2}}}\right| \cdot \mid \overline{V_{V}^{13}}\right\} \ldots \mid \overline{V_{V}^{1 s}}\right\}\right)_{V}
$$

ed a questa relazione, tenuto conto del n. 5, a') e del n. 8, si può dare la forma:

$$
\left.\mid V_{V}^{\Delta}\right\}=\left(\left(\left\{\overline{V_{V}^{18}} \mid V^{\Delta}\right)_{V} \cdot\left(\left|\overline{V_{V}^{13}}\right| \nabla^{\Delta}\right\rangle_{V} \ldots\left(\left|\overline{V_{V}^{i s}}\right| V^{\Delta}\right)_{V}\right)_{V^{\Delta}}\right. \text {. }
$$


D'altro tanto, poggiando sulle (1!, (4), $\left(5_{62}\right)$, si vede che è

$$
\left|\overline{\nabla_{V}^{12}}\right|=\left|\nabla_{V^{1} \times V^{2}}^{12}\right| \times \nabla^{3} \times \ldots \times \nabla^{s}
$$

sicchè, in virtù di quanto si è detto dianzi sulla (5), risulta

e, parimenti,

$$
\left.\left(\mid \overline{V_{V}^{12}}\right\} \nabla^{\Delta}\right)_{V}=\left\{\nabla_{\mid 2\}}^{\Delta}\right\}
$$

$$
\left(\left\{\overline{\nabla_{V}^{1 i}}\right\} \nabla^{\Delta}\right)_{V}=\left\{\nabla_{\{i}^{\Delta}\right\} \quad \text { per } i=3, \ldots, s .
$$

Così la (6) fornisce l'equivalenza

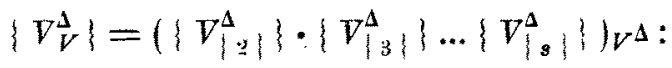

basta quindi trasformare i due membri di questa mediante la $Q$, per dedulne la (3).

\section{§ II. - Successioni canoniche e successioni covarianti d'immersione.}

66. Nel paragrafo precedente abbiamo ottenuto vari modi per costruire delle successioni invarianti sopra una data varietà algebrica irriducibile e non singolare, che allora chiamavamo $V^{\mathrm{t}}$, ma che ora designeremo con $V$, ed abbiamo anche mostrato come ciascuna di tali successioni possa esprimersi in funzione della $|V|$, definita quale la successione covariante d'immersione di $\nabla$ pensata come varietà diagonale entro la varietà prodolto $V \times V$. La considerazione della $|V|$ riescirebbe priva di utilità qualora questa successione si riducesse alla $V, 0,0, \ldots$; ma così non è per varietà $V$ generiche di dimensione positiva, come risulterà da quanto diremo in seguito.

Nel presente numero, indicheremo fra l'altro due casi particolari notevoli, costituenti i soli esempi nel presente ordine d'idee conosciati prima della pubblicazione del lavoro di B. SEGRE [9]. Questi esempi mostrano già l'opportunità di sostituire alla considerazione della suddetta successione invariante $\{\nabla\}$, quella dell' inversa della sua alternante. Porremo dunque

$$
\left.\mid V^{*}\right\}=\{\overline{\tilde{V}}\},
$$

e chiameremo $\left|V^{*}\right|$ la successione canonica della varietà $V$; corrispondentemente avremo da considerare su $\nabla$ la varietà invariante $V_{i}^{*}$ (per $i=1,2, \ldots, v$ ), che sarà una varietà pura (virtuale) di dimensione $v-i$, definita da $V$ a meno di un' equivalenza, da chiamarsi la $i$-ma varietà canonica, od anche la variet̀̀ canonica di dimensione $v-i$ o d'indice $i$, relativa a $V$ (rispettivamente ipersuperficie canonica o gruppo canonico di $\nabla$, se $i=1$ od $i=v$ ).

In particolare, per $i=v$, la (1) fornisce

$$
V_{v}^{*}=(-1)^{v} \tilde{V}_{v}=(-1)^{v} \tilde{V}_{V \times V, v} .
$$


E poichè $V \times V$ ha dimensione $2 v$, cosi - in base al teorema generale del n. 23 - da qui consegue la

$$
\nabla_{v}^{*}=(-1)^{v}\left(\nabla^{[2]}\right)_{V \times V} .
$$

Pertanto, con le definizioni dianzi adottate,

Un gruppo canonico della varietà $V$, preso col proprio segno o mutato di segno secondochè $l a$ dimensione $v$ di $\nabla$ è pari o dispari, risulta equivalente ad un gruppo caratteristico della $V$ pensata come varietà diagonale entro la varietà $\nabla \times \nabla$.

Nei casi $v=1$ e $v=2$, questo risultato si ricollega alle proprietà conosciute a cui alludevamo in principio, e sulle quali avremo poi occasione di di ritornare (n. 90). E precisamente, se $V$ è una curva di genere $p$, il confronto di quel risultato cul significato funzionale del principio di corrispondenza per le corrispondenze a valenza sopra una curva algebrica $\left({ }^{(1)}\right)$ mostra che:

La nozione di gruppo canonico $V_{1}^{*}$ di una curva $V$, secondo la nostra definizione, coincide con quella di gruppo canonico di $V$ nel senso classico (sicchè $\nabla_{1}^{*}$ consta di $2 p-2$ punti, se $p$ è il genere di $V$ ).

Se $V$ è una superficie, di eui $I$ denoti l'invariante di Zeuthen-Segre, basta rammentare il significato topologico della serie di Severi $\left({ }^{22}\right)$, per dedurre dal primo enunciato del presente numero che

La nozione di gruppo canonico $\nabla_{2}^{*}$ di una superficie $\nabla$, secondo la nostra definizione, coincide con quella di gruppo (di $I+4$ punti) della serie di equivalenza di Severi. $\left({ }^{(2)}\right)$.

67. Il legame intercedente fra varietà canoniche e varietà covarianti d'immersione può venire svincolato dalla considerazione delle varietà diagonali, come prova il seguente

Teorema. - Se $P$ e V sono due varietà irriducibili, la prima delle quali appartenga alla seconda, sussiste la

$$
\left|P^{*}\right|=\left(\left|\bar{P}_{V}\right| \cdot\left|V^{*}\right|\right)_{V}
$$

dove (secondo le convenzioni dei nn. 6,66) $\left\{P^{*}|,| \nabla^{*} \mid\right.$ indicano le successioni canoniche di $P, V$, e $\left|\bar{P}_{V}\right|$ denota l'alternante della successione covariante d'immersione di $P$ in $V$.

(21) Cfr. al riguardo Severi [6], n. 141.

(22) Tale significato topologico è di Comessater [1], il quale si è però limitato a trat. teggiarne una dimostrazione. Dimostrazioni compiute di altro tipo, furono poi date dal Severi [6], nn. 145, 154.

${ }^{(23)}$ Nuove dimostrazioni degli ultimi due enunciati, si avranno come casi particolari dal § III del Capitolo settimo. 
Identifichiamo, com'è lecito le $\nabla, P$ con le varietà diagonali dei prodotti

$$
V^{\prime}=V \times V, \quad P^{\prime}=P \times P .
$$

Risulterà allora

$$
P \subset V \subset \nabla^{\prime}, \quad P \subset P^{\prime} \subset V^{\prime}
$$

ed inoltre, a norma della $\left(1_{6 \sigma}\right)$, si avrà :

$$
\left\{V^{*}\right\}=\left\{\tilde{\bar{V}}_{V^{r}}\right\}, \quad\left\{P^{*} \mid=\left\{\tilde{\bar{P}}_{P^{\prime}}\right\}\right. \text {. }
$$

In forza del teorema dell' appartenenza (n. 18), dalle (3) si traggono le:

$$
\left|P_{V^{\prime}}\right|=\left(\left.\left|P_{V}\right| \cdot\left|V_{V^{\prime}}\right|\right|_{V}, \quad\left|P_{V^{\prime}}\right|=\left(\left|P_{P^{\prime}}\right| \cdot\left|P^{\prime} V^{\prime}\right| P_{P^{\prime}} .\right.\right.
$$

$D^{\prime}$ altro canto, in base alla (2) ed al n. 63 , risulta;

$$
\left.P^{\prime}{ }_{V^{\prime}}=\nmid(P \times P)_{V \times V}\right\}=\left\{P_{V}|\times| P_{V}\right\} \text {. }
$$

Basta quindi confrontare fra loro le (5) e servirsi delle (2), (4), (6), per ottenere l'equivalenza

$$
\left.\left(\mid P_{V}\right\} \cdot\left|\tilde{\bar{V}}^{*}\right|\right)_{V}=\left(\left|\tilde{\bar{P}}^{*}\right| \cdot\left(\left|P_{V}\right| \times \mid P_{V}\right\}\right)_{P \times P} .
$$

Notiamo ora che tanto un membro che l'altro della (7) risulta una successione appartenente alla varieta $P \times \nabla$. Ha quindi senso prendere i prodotti - entro questa varieta - di quei due membri per la successione $\left\{\tilde{P}_{V}\right\} \times V$. Cosi facendo, e tenendo conto della proposizione finale del n. 58, il primo membro della (7) si riduce a:

$$
\left.\left(\left(\mid P_{V}\right\} \cdot\left|\tilde{P}_{V}\right|\right)_{P} \cdot\left|\overline{\tilde{V}}^{*}\right|\right)_{V}=\left(P \cdot\left\{\tilde{\bar{V}}^{*} \mid\right)_{V}\right. \text {. }
$$

Il secondo membro invece, in virtù del n. 5, a) e del n 8 , diventa

$$
\left.[\} \tilde{\bar{P}}^{*}\right\} \cdot\left(\mid P_{V} \times\left\{P_{V}\right\}\right) \cdot\left(() \tilde{P}_{V}\{\times \nabla)(P \times P)_{P \times V}\right]_{P \times P}
$$

e poichè, in virtù del n. 60 , si ha

$$
\begin{gathered}
\left(\left(\left\{\tilde{P}_{V}\{\times V)(P \times P)\right)_{P \times V}=\left(\left\{\tilde{P}_{V}: P\right)_{P} \times(V P)_{V}=\left\{\tilde{P}_{V}\{\times P,\right.\right.\right.\right. \\
\left(( \{ P _ { V } \{ \times \} P _ { V } \} ) \cdot \left(\left\{\tilde{P}_{V}\{\times P)\right)_{P \times P}=\left(\left\{P_{V} \cdot \cdot\left\{\tilde{P}_{V}\right\}\right)_{P} \times\left(\left\{P_{V}\right\} \cdot P\right)_{P}=P \times \mid P_{V}\right\}\right.\right.
\end{gathered}
$$

cosi, usufruendo ancora della proposizione finale del n. 58, vediamo che la (9) può anche scriversi nella forma

$$
\left(\left\{\tilde{\bar{P}}^{*}\right\} \cdot\left(P \times\left\{P_{V}\right\}\right)_{P \times P}=\left(\left\{\tilde{\bar{P}}^{*}\right\} \cdot\left\{P_{V}\right\}\right)_{P}\right.
$$

In virtù di ciò che precede, questa successione deve uguagliare la (8). Si ha pertanto

$$
\left(P \cdot \mid \tilde{\bar{V}}^{*}\{)_{V}=\left(\left\{\tilde{\bar{P}}^{*}\right\} \cdot\left\{P_{V}\right\}\right)_{P}\right.
$$


Si rilevi infine che ognuno dei due membri della (10) è una successione di sostegno $P$. Moltiplicando ciascuno di essi per $\left\{\bar{P}^{*}\right\}-$ entro $P$ - ed applicando la $\left(5_{8}\right)$, ne deduciamo la

$$
\left.\left(\left|\bar{P}^{*}\right| \cdot\left|\tilde{\bar{V}}^{*}\right|\right)_{v}=\mid P_{V}\right\} .
$$

Ne consegue la (1), con l'uguagliare fra loro le successioni alternanti (n. 6) dei due membri della (11), e moltiplicare poi - entro $V$ - i due membri della relazione cosi ottenuta per $\left\{\nabla^{*}\right\}$.

\section{$\S$ III. - Successioni canoniche nei prodotti diretti.}

68. Ci proponiamo di stabilire il seguente

Teorema. - Sappiamo che una qualunque varietà algebrica $V$, irriducibile e non singolare, può venir identificata con la varietà diagonale della varietà $W$ che si ottiene facendo $i l$ prodotto diretto $d i \quad V$ un arbitrario numero $s(\geq 2) d i$ rolte per se stessa (n. 57). Ebbene, la successione canonica di $W$ sega su tale varielà diagonale la potenza s-ma della successione canonica a questa relativa.

Dalla $V \subset W$ si trae infatti anzitutto, in virtù del n. 67, la

$$
\left|\nabla^{*}\right|=\left(\left|\vec{\nabla}_{W}\right| \cdot\left|W^{*}\right|\right)_{W}
$$

D'altro canto, in base ai nn. 65,66 , risulta

$$
\left.\mid V_{W}\right\}=\mid \tilde{\bar{\nabla}}^{*}\{[s-1] \text {, }
$$

eppertanto :

$$
\left.\left\{\nabla^{*}\right\}^{[s-1]}=\mid \tilde{\bar{V}}_{W}\right\} \text {. }
$$

Sia i due membri della (1) che quelli della (2) sono successioni aventi per sostegno la varietà diagona!e $V$ di $W$. Basta quindi moltiplicare a membro a membro le (1), (2) - entro a $\nabla$ - per dedurne l'equivalenza

$$
\left.V^{*}\right\}^{[s]}=\left(V \cdot\left|W^{*}\right|\right)_{W}
$$

e questa prova l'asserto.

69. Dimostremo ora che

La successione canonica di un qualunque prodotto diretto

$$
W=M \times N \times \ldots \times P
$$

viene fornita dall' equivalenza

$$
\left|W^{*}\right|=\left\{M ^ { * } \left\{\times\left\{N^{*}\right\} \times \ldots \times\left\{P^{*}\right\} .\right.\right.
$$

Posto per abbreviare

$$
W^{\prime}=W \times W, M^{\prime}=M \times M, N^{\prime}=N \times N_{2}, \ldots, P^{\prime}=P \times P,
$$


la (1) fornisce

$$
W^{\prime}=M^{\prime} \times N^{\prime} \times \ldots \times P^{\prime},
$$

e le $W, M, N, \ldots, P$ - legate dalla (1) - possono venir ordinatamente identificate con le varietà diagonali delle $W^{\prime}, M^{\prime}, N^{\prime}, \ldots, P^{\prime}$. Il teorema del n. 63 allora porge:

$$
\left.\mid W_{W^{\prime}}\right\}=\left|M_{M^{\prime}}\right| \times \mid N_{N^{\prime}}\left\{\times \ldots \times\left\{P_{P^{\prime}}\right\} .\right.
$$

Inoltre, in virtù del n. 66, valgono le

$$
\left|W_{W^{\prime}}\right|=\left\{\tilde{\bar{W}}^{*}\right\},\left|M_{M^{\prime}}\right|=\left\{\tilde{\bar{M}}^{*}\right\}, \ldots,\left\{P_{P^{\prime}}\right\}=\left\{\tilde{\bar{P}}^{*}\right\}
$$

Ne consegue che è

$$
\left.\mid \tilde{\bar{W}}^{*}\right\}=\left\{\tilde{\bar{M}}^{*}|\times| \tilde{\bar{N}}^{*}|\times \ldots \times| \tilde{\bar{P}}^{*} \mid\right.
$$

Basta quindi uguagliare le inverse delle alternanti delle due successioni - di sostegno $W$ - costituenti i due membri di questa relazione, facendo uso del n. 61 , per ricavare la (2).

È interessante rilevare come il teorema del n. 68 possa anche dedursi quale semplice corollario del teorema testè stabilito. A tal nopo si definisca come dianzi la varietà $W$ con la (1), supponendo però che le $M, N, \ldots, P$ siano $s$ copie di una stessa rarietà $V$; ne seguirà così la (2). Si seghino allora i due membri di questa relazione con la varieta diagonale di $W$ : ció fornisce senz'altro il teorema del n. 68 , quando si faccia uso del n. 58.

\section{$\S$ IV. - Teoremi d'aggiunzione ed altre conseguenze.}

70. Sia $P$ una sottovarietà irriducibile e non singolare di $V$, interse. zione completa di $s=v-p$ ipersuperficie $A^{1}, A^{2}, \ldots, A^{s}$ di $V$. Sappiamo che allora (nn. 17, 7) risulta

$$
\bar{P}_{V, j}=P \cdot \tilde{\bar{\nabla}}_{j}(A)
$$

dove $\tilde{\bar{\nabla}}_{f}(A)$ denota la somma di tutti i prodotti delle $A$ prese con ripetizione a $j$ a $j$. Il teor. del n. 67 porge quindi senz'altro che:

La i-ma varietà canonica della suddetta varietà $P=\left(A^{ \pm} A^{*} \ldots A^{s}\right)_{V}$ si esprime mediante l' equivalenza

$$
P_{i}^{*}=P \cdot \sum_{j=0}^{i} \tilde{\bar{V}}_{j}(A) \nabla_{i-j}^{*} \quad(i=0,1,2, \ldots),
$$

dove le $\nabla^{*}$ sono le varietà canoniche di $\nabla$ e $\tilde{\bar{V}}_{j}(A)$ uguaglia la somma di tutti $i$ prodotti delle $A$ prese a $j$ a $j$ con ripetizione. 
In particolare, se $P=A$ è un'ipersuperficie di $\nabla$, utilizzando la (1) prima per il valore $i>0$ dell'indice e poi per il valore $i-1$, si ottiene:

$$
\begin{aligned}
A_{i}^{*} & =A \cdot \sum_{j=0}^{i} A^{[j]} V_{i-j}^{*}=A \cdot\left(V_{i}^{*}+A \cdot \sum_{j=0}^{i-1} A^{\left.[j] V_{i-j-1}^{*}\right)}=\right. \\
& =A \cdot\left(\nabla_{i}^{*}+A_{i-1}^{*}\right) .
\end{aligned}
$$

Le varietà $A_{\{i\}}$ definite a meno di un' equivalenza dalla

$$
A_{\{i\}}=\nabla_{i}^{*}+A_{i-1}^{*}
$$$$
(i=1,2, \ldots),
$$

sono varieta pure di dimensione $v-i$, associate covariantivamente ad $A$ entro a $\nabla$, e le diremo le $i$-me varietà aggiunte ad $A$ in $V$. Allora le (2), (3) forniscono il teorema dell'aggiunzione generalizzato:

$$
A_{i}^{*}=\left(A \cdot A_{|i|}\right) v
$$

enunciabile dicendo che su di un'ipersuperficie $A$ non singolare di $V$, le $i$-me varietà aggiunte di A segano varietà canoniche d'indice $i$.

Un' ipersuperficie aggiunta di $A$ in $\nabla$ nel senso olassico fottenibile p. es. sottraendo $v A$ dal sistema lineare jacobiano di $|A|)$. verra denotata secondo l'uso con $A^{\prime}$; indicheremo poi con $X(\nabla), X(A)$, ecc., un'ipersuperficie can onica (virtuale ed impura) di $\nabla, A$, ecc., nel senso classico. È ben noto che, se il sistema lineare $|A|$ soddisfa ad opportune condizioni di genericita, l'aggiunto $\left|A^{\prime}\right|$ può venir caratterizato mediante la proprietà di segare sulla generica $A$ ipersuperficie canoniche (classiche):

$$
X(A)=\left(A A^{\prime}\right)_{V}
$$

e che vale inoltre il te orema dell'aggi unzione, esprimibile nella forma:

$$
A^{\prime}=A \dashv X(\nabla)
$$

equivalente alla

$$
(A+B)^{\prime}=A^{\prime}+B=A+B^{\prime} .
$$

Ebbene, dimostreremo che:

Le nozioni classiche dianzi richiamate coincidono con casi particolari di quelle precedentemente poste nel presente numero, risultando precisamente (per ogni varietà $V$ irriducibile e non singolare di dimensione $v>0$, e per ogni ipersuperficie $A$ non singolare di $V$ ):

$$
X(V)=\nabla_{1}^{*}
$$

$$
A^{\prime}=A_{\{1 \mid} \text {. }
$$

In virtù del penultimo teorema del n. 66, la (7) sussiste se $\nabla$ è una curva, e cioè per $v=1$. Basterà dunque dimostrare la (7) nell'ipotesi che sia $v \geq 2$, ammettendo induttivamente ch'essa valga per varietà di dimensione $v-1$, talchè si avrà

$$
X(A)=A_{1}^{*}
$$


Ora, in virtù di quest' ultima relazione, le (4), (5) mostrano che le ipersuperficie $A^{\prime}$ ed $A_{\{1\}}$ di $V$ segano la generica $A$ lungo varietà equivalenti ; esse debbono dunque risultare fra loro equivalenti, a norma di un noto eriterio d'equivalenza di Severi, il che dímostra la (8). Siccome la (3) per $i=1$ riducesi alla

$$
A_{\{1\}}=\nabla_{1}^{*}+A \text {, }
$$

cosi basta raffrontare questa relazione con la (6), e tener conto della (8), per dedurre la (7).

71. Poichè la successione covariante d'immersione di una varietà in un'altra può venir espressa mediante le successioni canoniche inerenti alle due varietà (n. 67), cosi i risultati ottenuti precedentemente sulle succes. sioni covarianti d'immersione possono agevolmente venir trasformati in guisa da far comparire dappertutto in vece di quelle soltanto delle successioni canoniche. In successivi numeri del presente paragrafo daremo vari esempi in proposito; altri esempi consimili potrebbero venir oitenuti dal Lettore senza difficoltà.

Ora incominciamo col mostrare come, poggiando sul n. 67, si possa giungere assai rapidamente al teorema dell'appartenenza, già stabilito in modo diretto ma meno immediato nel $\mathrm{n}$. 18; il che costituirà un opportuno controllo. A tal uopo, supponiamo che valgano le $\left(2_{18}\right)$; a norma della $\left(11_{67}\right)$ ne conseguono le

$$
\left.\left\{P_{M}\right\}=\left(\mid \bar{P}^{*}\right\} \cdot\left\{\tilde{\bar{M}}^{*}\right\}\right)_{M}, \quad \mid M_{V} i=\left(\left\{\bar{M}^{*} ! \cdot i \tilde{\bar{V}}^{*}\right\}\right)_{V} .
$$

Avuto anche riguardo alle $\left(5_{8}\right),\left(11_{67}\right)$, da qui si trae ohe è:

$\left(\left\{P_{M}\right\} \cdot\left\{M_{V} !\right)_{M}=\left(\left(\left\{\overline{P^{*}} ! \cdot\left|\tilde{\bar{M}}^{*}\right| \cdot\left|\bar{M}^{*}\right|\right)_{M} \cdot\left\{\tilde{\overline{V^{*}}} \mid\right)_{V}=\left(\left\{\overline{P^{*}}|\cdot| \tilde{V^{*}} \mid\right)_{V}=\left\{P_{V}\right\}\right.\right.\right.\right.$;

e ciò appunto dimostra la $\left(3_{18}\right)$.

79. Prese in $V$ due sottovarietà $P$ e $V^{\prime}$, poniamo per abbreviare:

$$
P^{\prime}=\left(P V^{\prime}{ }_{V}, \quad\left\{P^{* \prime}\right\}=\left(\left\{P^{*}\right\} \nabla^{\prime}\right)_{V}\right. \text {. }
$$

Allora, poggiando sulla $\left(1_{5 \tau}\right)$ e sul teorema della sezione (n. 19), otteniamo:

$$
\left\{P^{* \prime}\right\}=\left(\left\{\bar{P}_{V}\right\} V^{\prime} \cdot\left\{\nabla^{*}\right\}\right)_{V}=\left(\left\{\bar{P}_{V}^{\prime}\right\} \cdot\left\{V^{*}\right\}\right)_{V}
$$

Basta quindi applicare la $\left(11_{67}\right)$, per dedurre la seguente modificazione intrinseca di quel teorema della sezione:

$$
\left\{P^{* *}\right\}=\left(\left(\left\{P^{*}\right\} \cdot\left\{\tilde{V}^{*}\right\}\right)_{V^{\prime}} \cdot\left\{V^{*}\right\}\right)_{V} .
$$

La (1) potrebbe anche venir stabilita come corollario della formula (3), a cui giungeremo più sotto con opportuna modifica del teorema del pro. 
dot to (n. 20). In base a quest' ultimo, assunto:

$$
R=(M N \ldots P)_{V},
$$

risulta :

$$
\left\{R_{V}\right\}=\left(\left\{M_{V}\right\} \cdot\left\{N_{V}\right\} \ldots\left\{P_{V}\right\}\right)_{V} .
$$

In virtù della $\left(11_{67}\right)$, si ha quindi

$$
\left.1\left\{\bar{R}^{*}\right\} \cdot\left\{\tilde{\bar{V}}^{*}\right\}\right)_{V}=\left(\left(\left\{\bar{M}^{*}\right\}\left\{\tilde{\overline{V^{*}}}\right\}\right)_{V^{\prime}} \cdot\left(\left\{\tilde{\bar{N}}^{*}\right\} \mid \tilde{\bar{V}}^{*}\right\}\right)_{V} \ldots\left(\left\{\bar{P}^{*}\right\}\left\{\tilde{\bar{V}^{*}}\right\}\right)_{V V} ;
$$

da qui, prendendo le alternanti dei due membri, moltiplicando ambo i membri per $\left\{V^{*}\right\}$ e ricordando la $(2)$, otteniamo la

$$
\left\{(M N \ldots P)_{V}^{*}\right\}=\left(\left.\left\{M^{*}\right\} \cdot\left\{N^{*}\right\} \ldots\left\{P^{*}\right\} \cdot\left\{\tilde{V}^{*}\right\}^{[s-1]}\right|_{V},\right.
$$

dove $s$ denota il $\mathrm{num} \theta \mathrm{ro}$ delle varietà $M, N, \ldots, P$ di $V$ che cosi intervengono.

Nel caso particolare in cui ciascuna delle $M, N, \ldots, P$ sia un'ipersu perficie di $V$ la (3) si riduce alla $\left(1_{\gamma_{0}}\right)$, com' ̀ subito visto applicando le $\left(2_{7_{0}}\right)$.

73. Rifacciamoci ora alle considerazioni del n. 25. Se $C$ denota un'iper. superficie non singolare di $V$, soddisfacente alla $\left(7_{25}\right)$, a norma della $\left(1_{67}\right)$ risulta

$$
C_{l}^{*}=\sum_{i=0}^{l} \bar{C}_{i} V_{l-i}^{*}
$$

d'altro canto, $\bar{C}_{i}$ si esprime con la $\left(8_{25}\right)$, ossia - sotto forma più esplicita mediante la

$$
\bar{C}_{i}=\sum_{(M)} c M_{\{i\}}=\sum_{\mid M)} c \bar{M}_{i-k+1}
$$

dove i coefficienti $c$ sono interi positivi e le $M$ sono monomi nelle $A$ del tipo $\left(2_{25}\right)$, di cui $k$ denoti il grado, tali che

$$
f(A)=\underset{M}{\Sigma} c M
$$

costituisca lo sviluppo in serie di potenze delle $A$ della funzione razionale $\left(6_{z_{5}}\right)$.

Dalle (1), (2) si trae:

$$
C_{l}^{*}=\sum_{(M)} c \sum_{i=0}^{l} \bar{M}_{i-k+1} \nabla_{l-i}^{*}=\sum_{(M)} c c_{j=0}^{l-k+1} \bar{M}_{j} \nabla_{l-k-j+1}^{*} ;
$$

sicchè, ancora in forza della $\left(1_{\sigma 7}\right)$, risulta

$$
C_{l}^{*}=\sum_{(M)} c M_{l-k+1}^{*} \quad(l=0,1,2, \ldots) .
$$

E si noti che il primo membro e ciascuno degli addendi a secondo membro nella (4) hanno di fatto la stessa dimensione $v-l-1$. Questa condizione basta per determinare gli indici inferiori a secondo membro, una volta che sia fissato l'indice $l$ a primo membro, ciò che permette - con ovvio 
significato dei simboli - di scrivere la (4) sotto la seguente forma compatta:

$$
\left\{C^{*}\right\}=\sum_{(M)} c\left\{M^{*}\right\}
$$

od anche, avuto riguardo alla (3), nella forma

$$
\left\{C^{*}\right\}=\left\{f(A)^{*}\right\} \text {. }
$$

La (5) fornisce la successione canonica di un'ipersuperficie $O$ di $V$ che equivalga alla somma di $s$ ipersuperficie $A^{1}, A^{2}, \ldots, A^{s}$ assegnate; più precisamente, mediante la (5), ogni varietà di quella successione si esprime come una combinazione lineare - a coefficienti interi non negativi - delle varietà canoniche di ugual dimensione inerenti alle $A$ ed alle varietà ottenute inter. secando le $A$ ripetutamente fra loro. Così, ad esempio, tenuto conto della $\left(9_{25}\right)$, si ha subito che

$L^{\prime}$ ipersuperficie canonica di $C=A^{1}+A^{z}+\ldots+A^{s}$ si esprime con la

$$
C_{1}^{*}=\Sigma\left(A^{i}\right)_{1}^{*}+2 \Sigma\left(A^{i} A^{j}\right)
$$

la varietò canonica d'indice due di $C$ è data dalla

$$
C_{2}^{*}=\Sigma\left(A^{i}\right)_{2}^{*}+2 \Sigma\left(A^{i} A^{j}\right)_{1}^{*}+3 \Sigma\left(A^{i} A^{j} A^{h}\right)+\Sigma\left(C A^{i} A^{j}\right),
$$

ecc., dove le somme vanno estese alle combinazioni semplici dei numeri $1,2, \ldots, s$, di classe uguale al rispettivo grado nelle $A .\left({ }^{4}\right)$

Inoltre, poggiando su quanto precede e sulla $\left(3_{29}\right)$, si vede che:

La varielà canonica d'indice $i$ del multiplo secondo $s$ di un' ipersuperficie A si esprime con l' equivalenza:

$$
(s A)_{i}^{*}=s(A)_{i}^{*}+s(s-1)\left(A^{[2]}\right)_{i-1}^{*}+s(s-1)^{2}\left(A^{[?]}\right]_{i-2}^{*}+s(s-1)^{3}\left(A^{[4]}\right)_{i-3}^{*}+\ldots
$$

I risultati precedenti rientrano come casi particolari in aliri, ottenibili similmente senza difficoltà dal n. 29. Di più, servendosi del n. 30 , si può del pari ricavare in modo immediato la snccessione canonica della dif. ferenza di due date ipersuperficie. Osserviamo infine che, poggiando sui successivi nn. 79,87 , dalle proposizioni suindicate se ne possono agevolmente ricavare altre consimili concernenti le varieià jacobiane generalizzate di un sistema lineare che sia somma o differenza di due o più altri.

74. Riprendiamo ora le considerazioni del Capitolo terzo, dal nostro nuovo punto di vista. Con le notazioni del n. 35, e tenuto conto del n. 67, vediamo subito che le $\left(1_{35}\right),\left(2_{35}\right)$ definiscono una medesima successione $\left\{P^{0}\right\}$; ed invero, sia dall' una che dall'altra di tali relazioni, esprimendo dappertutto

${ }^{(24)}$ Avuto riguardo ai risultati che verranno poi stabiliti nei nn. 89,94 , la proposizione testè ottenuta fornisce varie estensioni della classica formula di Noether per il genere di una curva comunque spezzata, assieme ai relativi significati funzionali. Per il caso particolare in cui sia $s=2$, efr. la formula (1.2) in Magphensos [1]. 
le successioni covarianti d'immersione che vi compaiono - in funzione delle successioni canoniche - mediante la $\left(11_{67}\right)$, si perviene alla:

$$
\left\{P^{0}\right\}=\left[\left(\ldots\left(\left\{\bar{P}^{*}\right\}\left\{\tilde{\bar{M}}^{* *}\right\}\right)_{M^{n}} \ldots\left\{\tilde{\bar{M}}^{s *}\right\}\right)_{M^{s}} \cdot\left\{{\overline{V^{*}}}^{*[s-1]}\right]_{V} .\right.
$$

Non v'è ora che da rifarsi ai nn. $35,37,40$ e da applicare la $\left(1_{14}\right)$, per ottenere il seguente

Teorema. - Se $M^{1}, M^{2}, \ldots, M^{s}$ sono $s(\geq 2)$ varietà di $V$ passanti semplicemente ed in modo generico per una varietà $P$ non singolare, e si suppone

$$
t=p+(s-1) v-\left(m^{1}+m^{2}+\ldots+m^{s}\right) \geq 0,
$$

allora l' equivalenza funzionale di $P$ nell' intersezione delle, $M^{\mathrm{t}}, M^{2}, \ldots, M^{8}$ in $\nabla$ $\grave{e}$ data dalla varietà $P_{t}^{0} d^{\prime}$ indice $t$ della successione (1), il che val quanto dire che l' intersezione $Q$ delle $M^{1}, M^{z}, \ldots, M^{s}$ residua a $P$ si esprime con la:

$$
Q=\left(M^{\prime} M^{2} \ldots M^{s}\right)_{V}-P_{t}^{0} \text {. }
$$

Quest'intersezione $Q$ residua, ha dimensione $q=p-t e$, se $q>0$, si ap. poggia a $P$ lungo una variotà $R-$ di dimensione $q-1$ - la quale soddisfa all'equivalenza

$$
R=P_{t+1}^{0} \text {. }
$$

Più generalmente, per ogni intero $k$ soddisfacente alle $0 \leq k \leq v-p-1$, risulta:

$$
R_{Q, k}=(-1)^{k}\left(R^{[k+1]}\right)_{Q}=P_{t+k+1}^{0},
$$

dove l'ultimo membro viene fornito intrinsecamente dalla (1).

75. Modificando le $\left(1_{48}\right),\left(2_{50}\right)$ con l'usufruire della $\left(11_{6}\right)$, vediamo che:

Se $P$ è una sottovarietà di $V$, dotata di un luogo $D$ di punti doppi impropri di dimensione regolare $d=2 p-v \geq 0$, tale luogo si esprime su $P$ mediante l'equivalenza

$$
D=\left(P^{[2]}\right)_{V}-(-1)^{v-p} \sum_{i=0}^{v-p}\left(\tilde{P}_{i}^{*} V_{v-p-i}^{*}\right)_{V}
$$

Qualunque sia $P$, una varietà $M$ di $V$ la cui dimensione $n$ soddisfi alle $p<m \leq 2 p-1, m<v$, e che passi genericamente per $P$, acquista di conseguenza su $P$ un luogo $\Delta$ di punti doppi (generalmente semplici per $P$ ), di dimensione $\delta=2 p-m-1$, espresso dalla

$$
\Delta=(-1)^{m-p+1} \sum_{i=0}^{m-p+1}\left(\tilde{P}_{i}^{*} M_{m-p-i+1}^{*}\right)_{M}
$$

oppure dalla

$$
\Delta=(-1)^{v-p} \sum_{i=0}^{v-p}\left(\tilde{P}_{i}^{*} M_{v-p-i}^{*}\right)_{M}-D
$$

nell' ipotesi che $M$ sia un' ipersuperficie di $V(m=v-1)$ e che $P$ possegga un luogo $D$ di punti doppi impropri [il quale allora viene fornito dalla (1)]. 


\section{$\S$ V. - Ampliamenti canonici entro un anello d'equivalenza.}

76. Dette $P$ e $V$ due qualunque varietì non singolari di cui la prima appartenga alla seconda, e tali che fra le loro dimensioni interceda la relazione $v \leq 2 p$, avuto riguardo ai nn. 22,23 ed alla $\left(11_{67}\right)$ risulta che:

Le varietà canoniche di due varietà $P, \nabla$, per le quali $P \subset \nabla, v \leq 2 p$, sono fra loro legate dalle equivalenze:

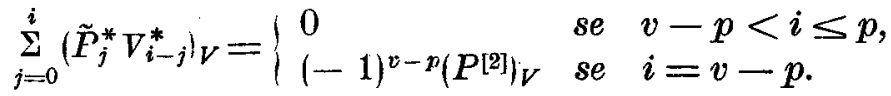

Possiamo facilmente - come controllo - verificare questo risultato nel caso particolare in cui $P$ sia la completa intersezione di $s=v-p \geq 1$ ipersuperficie di $\nabla$ :

$$
P=\left(A^{1} A^{2} \ldots A^{s}\right)_{V} .
$$

In tale ipotesi, la $\left(1_{\gamma_{0}}\right)$ fornisce:

$$
\tilde{P}_{j}^{*}=P \cdot \sum_{l=0}^{j}(-1)^{l} V_{l}(A) \tilde{V}_{j-l}^{*},
$$

dove le $V_{l}(A)$ sono le funzioni simmetriche elementari delle $A$ (definite in modo preciso nel n. 7). Ne consegue che è

$$
\sum_{j=0}^{i}\left(\tilde{P}_{j}^{*} V_{i-j}^{*}\right)_{V}=(-1)^{i} P \cdot \nabla_{i}(A) ;
$$

e queste implicano le (1), essendo (n. 7): $\nabla_{i}(A)=0$ se $i>s$ e $V_{s}(A)=P$.

77. Se $A$ denota un'ipersuperficie virtuale di $V$, da considerarsi virtual. mente non singolare, possiamo estendere la portata della $\left(2_{70}\right)$ e definire in ogni caso le varietà canoniche d'indice $i$ di $A$, assumendo

$$
A_{i}^{*}=A \cdot \sum_{j=0}^{i} A^{[j]} \nabla_{i-j}^{*} .
$$

Più generalmente, servendosi della $\left(1_{70}\right)$, si possono definire le varietà canoniche dei diversi indici di un' intersezione completa (virtuale):

$$
P=\left(A^{1} A^{z} \ldots A^{s}\right)_{V} .
$$

Da qui, tenuto anche conto del n. 73 , si perviene a definire le varietà cano. niche inerenti ad una qualunque sottovarietà virtuale di $\nabla$ (virtualmente priva di punti multipli). In particolare, acquista senso in ogni caso di parlare delle varietà canoniche di varietà canoniche $d i \quad V$, e sia queste varietà che le loro intersezioni hanno manifesto significato invariante. Ne consegue che il numero dei punti costituenti una siffatta varietà, nell'ipotesi ch'essa sia di dimensione nulla, risulta un invariante birazionale $d i V$. Ritorneremo più ampiamente nei nn. 94, 95 su questa osservazione. 
Si consideri ora un qualunque numero di varieta $M, N, \ldots$ di $V$, ed il sottoanello $\mathfrak{S B}$ di $\mathfrak{Z}_{V}$ da esse determinato. L'insieme delle varietá cano. niche inerenti alle singole varietà di $\mathfrak{T B}$ è un'ampliamento $\mathfrak{H B}^{*}$ di $\mathfrak{H B}$, a cui appartengono le varietà $M, N, M_{i}^{*}, N_{j}^{*}, \ldots$, il quale si dirà l'ampliamento canonico $d i \mathfrak{B}$. Relativamente ad esso possono porsi varie importanti questioni, come quelle di decidere se e quando $\mathfrak{B B}^{*}$ sia ancora un anello, o di dare condizioni affinche $\mathbb{B}^{*}$ coincida con $\mathfrak{B}$. In tale studio, dovranno giocare in modo essenziale i risultati dei nn. 72,73 ; ma non ci soffermiamo su esso, per non rendere eccessiva la mole del presente lavoro.

Ci limitiamo ad osservare in proposito che, in base alle ricerche di B. SEGRE [1] (p. 512), può dirsi che l'anello determinato dalle varietà canoniche di una varietà $\nabla$ a due o tre dimensioni, risulta chiuso di fronte agli ampliamenti canonici. Senza voler trattare qui la questione generale per una varietà $V$ di dimensione qualunque, daremo ora qualche esempio al riguardo.

Denotando $X=V_{1}^{*}$ un' ipersuperficie canonica di $V$ (n. 70), consideriamo una varieta caratteristica $P$ d'indice qualsiasi del sistema canonico $|X|$ :

$$
P=\left(X^{[s]}\right)_{V} \quad(1 \leq s \leq v-1) .
$$

A norma della $\left(1_{70}\right)$, le varietà canoniche di questa si esprimono con le

$$
P_{i}^{*}=P \cdot \sum_{j=0}^{i}\left(\begin{array}{c}
s+j-1 \\
j
\end{array}\right) X^{[j] \nabla_{i-j}^{*}} \quad(0 \leq i \leq v-s),
$$

ed appartengono quindi tutte all'ampliamento canonico dell'anello di base $\nabla$. In particolare, per $i=1$, la (2) - tenuto conto della (1) - fornisce la :

$$
P_{1}^{*}=(s+1) \cdot X^{[s+1]}
$$

e questa si riduce alla $P_{1}^{*}=v X^{[v]}$ se $s=v-1$, nel qual caso - in base alla (1) $-P$ ò una curva caratteristica del sistema canonico. Dunque:

Sopra una varietò di dimensione $v$, un gruppo canonico della curva caratteristica del sistema lineare delle ipersuperficie canoniche, equivale a volte un gruppo caratteristico di questo stesso sistema lineare $\left({ }^{25}\right)$.

78. Si ottengono subito altri risultati nell'indirizzo accennato nel numero precedente, assumendo - com' ̀े lecito - nella $\left(1_{76}\right)$ :

$$
P=V_{s}^{*} \quad(0<2 s \leq v) .
$$

(25) Cfr. B. Segre [2], n. 3. Dal punto di vista numerativo, il risultato funzionale ultimo ottenato fornisce l' uguaglianza $2\left(\Omega_{1}-1\right)=v \Omega_{0}$, mostrante che per $v$ dispari il grado $\Omega_{0}$ del sistema lineare canonico dev'esser pari; questa uguaglianza comprende, per $v=2$, 3, note formule di Noether e di Pannelli. Risultati numerativi in un ordine d'idee consimile, furono poi ottenuti da Todd e MaxweiL [1]. 
In tal guisa risulta: $p=v-s$, ond' '̀ $v \leq 2 p$, e la $\left(1_{76}\right)$ diventa :

$$
\left.\sum_{j=0}^{i} \widetilde{\left(\left(\nabla_{s}^{*}\right)_{j}^{*}\right.} V_{i-j}^{*}\right)_{V}= \begin{cases}0 & \text { se } s<i \leq v-s, \\ (-1)^{s}\left(V_{s}^{*[2]}\right)_{V} & \text { se } i=s .\end{cases}
$$

Le (1) porgono vari legami fra le varietò canoniche di $V$ e le varietà canoniche di tali varietà canoniche. Così, ad esempio, assumiamo $v \geq 3, s=1$, $i=2$, e poniamo $\operatorname{com} \theta$ dianzi

$$
X=\nabla_{1}^{*}
$$

In virtù del n. 5 valgono le:

$$
\tilde{X}_{1}^{*}=-X_{1}^{*}, \quad \tilde{X}_{2}^{*}=\left(X_{1}^{*[2]}\right)_{X}-X_{2}^{*} ;
$$

inoltre dalle $\left(1_{77}\right),\left(3_{77}\right)$ si trae

$$
X_{1}^{*}=2 X^{[2]}
$$

Pertanto, dopo facili riduzioni, la (1) fornisce l'equivalenza

$$
X_{2}^{*}=X\left(\nabla_{2}^{*}+2 X^{[2]}\right) \text {, }
$$

la quale mostra che una varietà canonica d'indice 2 di un'ipersuperficie canonica $X$ di $V$ pù̀ venir segata su $X$ dalla somma di una varietà canonica d'indice 2 di $V$ col doppio d'una prima varietà caratteristica di $|X|\left({ }^{26}\right)$.

\section{CAPITOLO SETIIMO}

\section{RAFFRONTI ED ESEMPI}

\section{§ I. - Varietà canoniche nel senso classico e varietà jacobiane.}

79. Sia $A$ una qualunque ipersuperficie abbastanza generale di $\nabla\left({ }^{27}\right)$ : ad esempio, la sezione di $\nabla$ con una forma del suo spazio ambiente. Allora, se $h$ denota un intero qualsiasi soddisfacente alle limitazioni $0 \leq h \leq v-1$, un generico sistema lineare $\infty^{h+1}$ appartenente totalmente ad $|A|$ ammette una varietà jacobiana, che denoteremo con $J^{h}(A)$, la quale è una varietà pura di dimensione $h$, definita da $A$ a meno di un'equivalenza $\left({ }^{28}\right)$. Una siffatta varietà può venir espressa funzionalmente con l'uso delle varietà canoniche - intese nel senso classico - al modo seguente $\left({ }^{29}\right)$.

Presa una qualunque varietà $W$ (irriducibile e non singolare), denoteremo con $X^{h}(W)$ una sua varieta canonica nel senso classico, di

(26) Per $v=3$, efr. B. Segre [1], formula [82].

(27) Il significato preciso di questa locuzione, viene implicitamente fornito dal perioro seguente del testo.

(28) Ciò si prova generalizzando gli sviluppi dati per $v=3$ in B. SEGRE [1], nn. 23, 26.

(29) Cfr. Eger [B], n. 20, J. A. Todd [7], n. 9. 
dimensione $h \leq w$, e porremo

$$
X^{h}(W)=0 \quad \text { se } h>w, \text { oppure se } h<0 .
$$

In particolare, in ogni caso risulta

$$
X^{m}(W)=W .
$$

Vale allora l'equivalenza

$$
J^{h}(A)=X^{h}\left((A+\nabla)^{[h+2])} \quad(h=0,1,2, \ldots),\right.
$$

che è una forma compatta per scrivere la

$$
J^{h}(A)=\sum_{j=0}^{h+2}\left(\begin{array}{c}
h+1-2 \\
i
\end{array}\right) X^{h}\left(A^{[i]}\right) \quad\left(A^{[0]}=1=\nabla\right) .
$$

Per $h=0$, la (3) [ossia la (4)] si scrive:

$$
J^{0}(A)=X^{0}(V)+2 X^{0}(A)+X^{0}\left(A^{[2]}\right),
$$

e porge un' equivalenza su $V$ legante il gruppo jacobiano di un generico fascio d'ipersuperficie $A$ ni gruppi canonici di $V$, di $A$ e di una varietà caratteristica $\left(A^{[2]}\right)_{V}$. Pertanto, detti ordinatamente $\delta, K_{v}, K_{v-1}, K_{v-z}$ i numeri dei punti di tali gruppi, vale la relazione

$$
\delta=K_{v}+2 K_{v-1}+K_{v-2} \text {. }
$$

Se $\nabla$ è una e urva di genere $p(v=1), A$ è un gruppo di un certo numero $a$ di punti di $V$ e, in base alle (1), (2), risulta

$$
K_{v-1}=a, \quad K_{v-2}=0 .
$$

Pertanto la (5) fornisce

$$
K_{1}=\delta-2 a=2 p-2 .
$$

Se $V$ è una superficie $\left(v=2\right.$, di cui $I_{2}$ denoti l'invariante di Zeuthen-Segre, $A$ e una curva, di cui $a$ e $p$ rispettivamente siano il grado ed il genere. Attualmente si ha

$$
K_{1}=2 p-2, \quad K_{0}=a,
$$

sicehè la (5) porge:

$$
K_{\mathrm{2}}=\delta-4 p-a+4=I_{2}+4 .
$$

Più in generale, se $v \geq 2$ ed $I_{v}$ denota l'invariante di Zeuthen-Segre di $V$, tenuto conto della definizione di questo invariante (cfr. C. SEGRE [1]), dalla (5) si ricava senz' altro:

$$
K_{v}=I_{v}+(-1)^{v} 2 v .
$$


80. Ci proponiamo ora di dimostrare il seguente

TEOREмa. - Fissati comunque due interi $h, s$ soddisfacenti alle

$$
0 \leq h \leq v-1, \quad s>v-h,
$$

e scelte ad arbitrio su $\nabla$ s ipersuperficie (abbastanza generali) $A_{1}, A_{2}, \ldots, A_{s}$, risulta:

$$
\begin{aligned}
X^{h}(V)= & \Sigma J^{h}\left(A_{i}\right)-\Sigma J^{h}\left(A_{i}+A_{j}\right)+\Sigma J^{h}\left(A_{i}+A_{j}+A_{l}\right)- \\
& -\ldots+(-1)^{s-1} J^{h}\left(A_{1}+A_{2}+\ldots+A_{s}\right),
\end{aligned}
$$

dove le somnie vanno rispettivamente estese a tutte le combinazioni semplici delle $A$ ad 1 ad 1, a 2 a $2, a 3$ a 3 , ecc. .

Consideriamo il polinomio nelle $A$ :

$$
\begin{gathered}
f(A)=\Sigma\left(1+A_{i}\right)^{h+2}-\Sigma\left(1+A_{i}+A_{j}\right)^{n+2}+\Sigma\left(1+A_{i}+A_{j}+A_{\imath}\right)^{n+2}- \\
-\ldots+(-1)^{s-1}\left(1+A_{i}+A_{i}+\ldots A_{s}\right)^{n+2}
\end{gathered}
$$

e scriviamolo, dopo aver sviluppato e ridotto $i$ termini simili, nella forma

$$
f(A)=c_{0}+\Sigma c A_{i_{1}}^{\left[k_{1}\right]} A_{i_{2}}^{\left[k_{2}\right]} \ldots A_{i_{n}}^{\left[k_{n}\right]}=c_{0}+\Sigma c P,
$$

dove le $c_{v}, c$ sono costanti numeriche, $i_{1}, i_{2}, \ldots, i_{\text {, denota una combinazione }}$ di $n$ fra $\mathrm{i}$ numeri $1,2, \ldots, s(1 \leq n \leq s)$, e gli esponenti $k_{i}$ sono interi positivi. In virtù della $\left(4_{79}\right)$, il secondo membro della (2) vale:

$$
c_{0} X^{h}(V)+\Sigma c X^{h}(P) \text {. }
$$

Ora, il confronto delle (3), (4) dà subito:

$$
c_{0}=\left(\begin{array}{l}
s \\
1
\end{array}\right)-\left(\begin{array}{l}
s \\
2
\end{array}\right)+\left(\begin{array}{l}
s \\
3
\end{array}\right)-\ldots+(-1)^{s-1}\left(\begin{array}{l}
s \\
s
\end{array}\right)=1 \text {. }
$$

Inoltre. posto per abbreviare

si ha:

$$
k=k_{1}+k_{2}+\ldots+k_{n}
$$

e

$$
n \leq k \leq h+2
$$

risulta una varietà pura di dimensione

$$
p=v-k \text {. }
$$

In virtù della $\left(1_{79}\right)$, se si vuole che non valga l'equivalenza $X^{h}(P)=0$ occorre supporre che si abbia $h \leq p$, e cioè :

$$
k \leq v-h .
$$

In quest' ipotesi, dalle (8), (1) si trae:

$$
1 \leq n \leq k<s .
$$


Allo scopo di determinare con quale coefficiente $c$ compare nella (4) il monomio $P$ fornito dalla (9), osserviamo che - fissato questo monomio (e quindi fissate le $i, k, n)$ in modo che valgano le (7), (8), (10) - il monomio stesso si ottiene unicamente dallo sviluppo di quegli addendi della (3) che sono della forma

$$
(-1)^{m-1}\left(1+A_{i_{1}}+A_{i_{2}}+\ldots+A_{i_{n}}+A_{i_{n+4}}+\ldots+A_{i_{m}}\right)^{n+2},
$$

dove $m$ denoti un qualunque intero soddisfacente alle $n \leq m \leq s$, ed $i_{n+1}, \ldots, i_{m}$ sia una combinazione arbitraria di classe $m-n$ degli $s-n$ numeri $1,2, \ldots, s$ distinti da $i_{1}, i_{2}, \ldots, i_{n}$. Risulta pertanto:

$$
c=\sum_{m=n}^{s}(-1)^{m-1}\left(\begin{array}{c}
s-n \\
m-n
\end{array}\right) \frac{(h+2) !}{k_{1} ! k_{2} ! \ldots k_{n} !(h+2-k) !}=0 .
$$

Avuto anche riguardo alla (6), la (5) equivale quindi alla $X^{h}(\nabla)$, ciò che prova la (2).

81. Applicando il teorema del numero precedente nell'ipotesi che si assuma

$$
A_{1}=A_{2}=\ldots A_{s}=A \text {, }
$$

per ogni $s>v-h$ e per ogni ipersuperficie $A$ abbastanza generale di $V$ otteniamo :

$$
X^{h}(V)=\left(\begin{array}{l}
s \\
1
\end{array}\right) J^{h}(A)-\left(\begin{array}{l}
s \\
2
\end{array}\right) J^{h}(2 A)+\left(\begin{array}{l}
s \\
3
\end{array}\right) J^{h}(3 A)-\ldots+(-1)^{s-1} J^{h}(s A) .
$$

Se ora sottraiamo la (1) a membro a membro dilla relazione che da essa si deduce ponendovi $s+1$ in luogo di $s$, vediamo che:

Per ogni ipersuperficie A (abbastanza generale) $d i V$ e per ogni $s>v-h$, sussistono le equivalenze:

$$
\sum_{i=0}^{s}(-1)^{i}\left(\begin{array}{l}
s \\
i
\end{array}\right) J^{h}((i+1) A)=0 \quad(h=0,1,2, \ldots, v-1) .
$$

In secondo luogo, denotiamo con $C$ un'altra ipersuperficie effettiva (sufficientemente generale) di $V$, ed applichiamo la $\left(2_{80}\right)$ facendovi

$$
A_{1}=A_{z}=\ldots=A_{s-1}=A, \quad A_{s}=C .
$$

Otteniamo così l'equivalenza:

$$
X^{h}(V)=\sum_{i=1}^{s-1}(-1)^{i-1}\left(\begin{array}{c}
s-1 \\
i
\end{array}\right) J^{h}(i A)+\sum_{i=0}^{s-1}(-1)^{i}\left(\begin{array}{c}
s-1 \\
i
\end{array}\right) J^{n}(i A+C) .
$$

Basta quindi sottrarre la (3) a membro a membro dalla (1) per dedurre la

$$
\sum_{i=0}^{s-1}(-1)^{i}\left(\begin{array}{c}
s-1 \\
i
\end{array}\right) J^{h}((i+1) A)=\sum_{i=0}^{s-1}(-1)^{i}\left(\begin{array}{c}
s-1 \\
i
\end{array}\right) J^{n}(i A+C)
$$

valida per ogni $s>v-h$, nell'ipotesi che $A$ e $C$ siano abbastanza generali. 
Confrontando la (3) con la relazione che da essa si deduce scrivenduvi $A$ e $C^{\prime}$ in luogo di $A$ e $C$, otteniamo

$$
\begin{gathered}
\sum_{i=1}^{s-1}(-1)^{i}\left(\begin{array}{c}
s-1 \\
i
\end{array}\right)\left(J^{h}(i A)-J^{h}\left(i A^{\prime}\right)\right)= \\
=\sum_{i=0}^{s-1}(-1)^{i}\left(\begin{array}{c}
s-1 \\
i
\end{array}\right)\left(J^{n}(i A+C)-J^{n}\left(i A^{\prime}+C^{\prime}\right)\right) .
\end{gathered}
$$

Dalla (4), ponendovi $s+1$ in luogo di $s$ ed utilizzando la (2), ricaviamo la

$$
\sum_{i=0}^{s}(-1)^{i}\left(\begin{array}{l}
s \\
i
\end{array}\right) J^{n}(i A+C)=0
$$

questa relazione vale anche se si pone $C-A$ in luogo di $C$, sicchè, scrivendo in pari tempo $i+1$ in luogo di $i$, risulta:

$$
0=\sum_{i=-1}^{s-1}(-1)^{i}\left(\begin{array}{c}
s \\
i+1
\end{array}\right)\left(J^{h}(i A+C)-J^{h}\left(i A^{\prime}+C^{\prime}\right)\right) \text {. }
$$

Sottraendo questa relazione a membro a membro dalla (5), otteniamo la:

$$
\begin{gathered}
\sum_{i=1}^{s-1}(-1)^{i}\left(\begin{array}{c}
s-1 \\
i
\end{array}\right)\left(J^{h}(i A)-J^{h}\left(i A^{\prime}\right)\right)= \\
=\sum_{i=0}^{s-1}(-1)^{i}\left(\begin{array}{c}
s-1 \\
i
\end{array}\right)\left[J^{h}((i-1) A+C)-J^{h}\left((i-1) A^{\prime}+C^{\prime}\right] ;\right.
\end{gathered}
$$

e si noti che il termine dell' ultima somma che proviene dal valore 0 dell'in dice $i$ va a zero, se si suppone $C-A=C^{\prime}-A^{\prime}$.

82. La $\left(2_{80}\right)$ permette di definire la varietà jacobiana (virtuale) $J^{h}(B)$, per ogni ipersuperficie $B$ di $V$ (anche virtuale). A tal nopo, consideriamo un intero $t \geq v-h$ e scegliamo su $\nabla$, com'è certamente possibile, un'ipersuperficie $A$ tale che - secondo l'accezione elementare richiamata nel n. $79-$ abbia senso considerare le varietà jacobiane $J^{h}$ relative a ciascuno dei sistemi lineari

$$
|A|,|2 A|, \ldots,|t A|,|A+B|,|2 A+B|, \ldots,|t A+B|
$$

(ognuno dei quali dovrà dunque in particolare risultare elfettivo ed almeno $\infty^{n+1}$. Se ora applichiamo la $\left(2_{81}\right)$ assumendovi

$$
s=t+1, \quad A_{1}=A_{2}=\ldots=A_{s-1}=A, \quad A_{s}=B,
$$

otteniamo una formula ove ogni addendo ha un preciso significato, tranne $J^{h}(B)$. Da essa traiamo:

$$
\left.J^{h}(B)=X^{h}(V)+\sum_{i=1}^{t}(-1)^{i}\left(\begin{array}{l}
t \\
i
\end{array}\right) \cdot J^{h}(i A)-J^{h}(i A+B)\right) ;
$$

ed è lecito assumere la (1) come definizi one di $J^{h}(B)$, purchè si dimostri che il secondo membro della (1) non dipende nè da $t(\geq v-h)$ nè da $A$, 
Ora, per provare che il sécondo membro della (1) non dipende da $t$, basta assicurarsi ch'esso equivale all'espressione che se ne deduce scrivendovi $s=t+1$ in luogo di $t$, e ciò̀ che risulta :

$$
\sum_{i=0}^{t}(-1)^{i}\left(\begin{array}{l}
t \\
i
\end{array}\right)\left[J^{n}((i+1) A)-J^{n}((i+1) A+B)\right]=0 .
$$

All' uopo non v'è che da osservare che, posto $C=A+B$, si può sempre supporre - con opportuna scelta di $A$ - che $C$ sia effettiva ed abbastanza generale; con cio la (2) certamente sussiste, in forza della $\left(4_{8}\right)$.

Per dimostrare che il secondo membro della (1) non dipende da $A$, occorre stabilire ch'esso uguaglia l'espressione che se ne deduce assumendovi, in luogo della $A$, un'analoga ipersuperficie $A^{\prime} d i \quad V$. Posto come dianzi $C=A+B$, similmente, $C^{\prime}=A^{\prime}+B$, talchè risulta $C-A=C^{\prime}-A^{\prime}$, ̀̀ lecito supporre che le ipersuperficie $A, A^{\prime}, C, C^{\prime}$ siano abbastanza generali in guisa che valga il risultato finale del n. 81 ; a questo appunto pruva quanto testè asserito.

Dalla suddetta definizione (1) della rarietà jacobiana virtuale $J^{n}(B)$, segue subito in particolare (per $B=0$ ) la notévole equivalenza:

$$
J^{h}(0)=X^{h}(V) \text {. }
$$

83. Una nuova interessante definizione possibile per le varietà canoniche $X^{h}(V)$ vien fornita dalla $\left(2_{8 n}\right)$. In base al $n .79$, si ha intanto che il secondo membro di questa relazione varia entro ad un sistema d'equivalenza, comunque varino le $s(>v-h)$ ipersuperficie $A_{1}, A_{2}, \ldots, A_{s}$ entro a sistemi lineari. Affinchè si possa asserire che quel sistema d'equivalenza è un sistema invariante di $\nabla$, basta provare ch'esso non muta se si cangiano arbitrariamente le ipersuperficie $A_{1}, A_{2}, \ldots, A_{s}$, il che risulta senz'altro vero, non appena si sappia che tale proprietò ha luogo quando si tengano fisse $s-1$ di quelle ipersuperficie e si muti la rimanente ad arbitrio.

Posto $t=s-1$, talchè dovrà supporsi

$$
t \geq v-h
$$

sarà dunque sufficiente mostrare che il secondo membro della $\left(2_{80}\right)$ si mantiene equivalente a se stesso quando vi si assuma

$$
A_{s}=A \quad \text { oppure } \quad A_{s}=A^{\prime} \quad\left(\text { con } A, A^{\prime} \text { ipersuperficie arbitrarie di } V\right)
$$

lasciando invece inalterate le altre $A_{i}(i=1,2, \ldots, t)$. Ciò val quanto dire che:

Nell'ipotesi che valga la (1), il sistema d'equivalenza determinato dalla

$$
\begin{gathered}
J^{h}(A)-\Sigma J^{h}\left(A+A_{i}\right)+\Sigma J^{h}\left(A+A_{i}+A_{j}\right)- \\
-\ldots+(-1)^{t} J^{h}\left(A+A_{1}+A_{2}+\ldots+A_{t}\right)
\end{gathered}
$$

(dove le somme vanno rispettivamente estese a tutte le combinazioni semplici degli indici $1,2, \ldots, t$ ad 1 ad 1, a 2 a 2 , ecc.), risulta indipendente da $A$. 
Non ci tratterremo a dare una dimostrazione diretta di questo fatto, indipendente cioè dalla $\left(2_{80}\right)$, ciò che porterebbe a stabilire ex novo la $\left(2_{81}\right)$, e quindi pure le formule $\left(3_{79}\right),\left(4_{79}\right)$ (di Eger e Todd) da cui eravamo partiti. Ci limiteremo invece ad osservare che, poichè il fatto testè asserito certamente sussiste, in forza della $\left(2_{80}\right)$, cosl per valutare l'espressione (2) si potrà in essa particolarizzare $A$ ad arbitrio, facendovi ad esempio (n. 82) $A=0$. In tal guisa, usufruendo delle $\left(2_{80}\right),\left(3_{87}\right)$ si ottiene che:

Se $t>v-h$, comunque si scelgano su $V$ le $t+1$ ipersuperficie $A, A_{1}$, $A_{2}, \ldots, A_{t}$, la varietà (2) risulta equivalente allo zero.

\section{§ II. - Le varietà canoniche negli spazi lineari.}

84. Esaminiamo ora particolareggiatamente il caso, specialmente importante per le applicazioni, in cui la varieta ambiente $V$ sia uno spazio proiettivo (complesso) [v], di dimensione $v$. Denoteremo secondo l'uso con [i] uno spazio subordinato di $[v]$, di dimensione $i(0 \leq i \leq v)$; inoltre, in conformità coi nn. 66, 79, le varietà canoniche di $[v]$ secondo la nostra definizione e nel senso classico si designeranno rispettivamente con $[v]_{i}^{*}$ e con $X^{h}([v])$ (queste avendo ordinatamente dimensione $v-i$ ed $h$ ). Vogliamo dimostrare che, per gli spazi proiettivi v'è identitò fra le due definizioni delle varietà canoniche, risultando

$$
[v]_{i}^{*}=X^{h}([v]) \quad \text { se } h=v-i .
$$

Incominciamo con l'osservare che, poichè - notoriannente $\left({ }^{30}\right)$ - la totalità delle varietà effettive di ordine e dimensione dati, di un [v], è contenuta totalmente in un unico sistema elementare di varieta virtuali dello stesso ordine e della stessa dimensione, così, detta $M$ una qualunque varietà pura contenuta in $[v]$, di ordine $\mu$ e dimensione $m$, risulta (in $[v])$ :

$$
M \cdot[v-m]=\mu \cdot[0], \quad M=\mu \cdot[m] .
$$

$\mathrm{Ne}$ consegue che per stabilire la (1), basta provare che $\grave{e}$

$$
\varphi(v, i)=f(v, h) \quad \text { se } h=v-i,
$$

dove $\varphi(v, i)$ ed $f(v, h)$ denotino rispettivamente l'ordine di $[v]_{i}^{*}$ e ai $X^{h}([v])$, e cioè ove le $\varphi, f$ si definiscano ponendo:

$$
[v]_{i}^{*}=\varphi(v, i) \cdot[v-i], \quad X^{h}([v])=f(v, h) \cdot[h] .
$$

85. Se $A$ denota un' ipersuperficie di $V=[v]$, d'ordine $a \geq 2$, in base al n. 79 ed a proprietà elementari di geometria proiettiva, la jacobiana $J^{h}(A)$ ha (dimensione $h$ ed) ordine

$$
\left(\begin{array}{l}
v+1 \\
h+1
\end{array}\right)(a-1)^{v-n}
$$

(30) Cfr. Severi [6], n. 43. 
Pertanto, con le notazioni del n. 84, identificando nelle ipotesi attuali gli ordini dei due membri della $\left(1_{s_{1}}\right)$ otteniamo

$$
\begin{gathered}
f(v, h)=\left(\begin{array}{l}
v+1 \\
h+1
\end{array}\right)\left(\begin{array}{l}
s \\
1
\end{array}\right)(a-1)^{v-n}-\left(\begin{array}{l}
s \\
2
\end{array}(2 a-1)^{v-n}+\left(\begin{array}{l}
s \\
3
\end{array}\right)(3 a-1)^{v-n}-\right. \\
-\ldots+(-1)^{s-1}(s a-1)^{v-n} !
\end{gathered}
$$

dove $s$ denoti un qualunque intero $>v-h$

L'espressione qui scritta entro graffe un polinomio in $a$ :

$$
(-1)^{v-n} \sum_{i=0}^{v-h}(-1)^{i}\left(\begin{array}{c}
v-h \\
i
\end{array}\right) k_{s, i} a^{i}
$$

dove, per abbreviare, si è posto:

$$
k_{s, i}=\left(\begin{array}{l}
s \\
1
\end{array}\right)-2^{i}\left(\begin{array}{l}
s \\
2
\end{array}\right)+3^{i}\left(\begin{array}{l}
s \\
3
\end{array}\right)-\ldots+(-1)^{s-1} s^{i}\left(\begin{array}{l}
s \\
s
\end{array}\right)
$$

Si ha quindi:

$$
\begin{aligned}
& k_{s, 0}=1 \\
& k_{s, i}=s\left(k_{s, i-1}-k_{s-1, i-1}\right)
\end{aligned}
$$

onde si trae induttivamente

$$
0=k_{s, 1}=k_{s, 3}=k_{s, 3}=\ldots .
$$

Risulta pertanto

$$
f(v, h)=(-1)^{v-n}\left(\begin{array}{l}
v+1 \\
h+1
\end{array}\right),\left({ }^{31}\right)
$$

sicchè la seconda delle $\left(3_{84}\right)$ fornisce:

$$
X^{h}([v])=(-1)^{v-h}\left(\begin{array}{l}
v+1 \\
h+1
\end{array}\right) \cdot[h] .
$$

Posto per abbreviare

$$
i=v-h, \quad X^{h}([v])=[v]_{i},
$$

la (2) può venire scritta nella forma

$$
[v]_{i}=(-1)^{i}\left(\begin{array}{c}
v+1 \\
i
\end{array}\right) \cdot[v-1]
$$

e mostra che le $[v]_{0}=[v],[v]_{1},[v]_{2}, \ldots$ formano una successione di sostegno $[v]$ (n. 6). Consideriamo la successione di sostegno [v] formata dalle varietà :

$$
[v]_{j}^{\prime}=\left(\begin{array}{c}
v+j \\
j
\end{array}\right) \cdot[v-j] \quad(j=0,1,2, \ldots)
$$

(3i) In particolare: $f(v, 0)=(-1)^{v}(v+1)$; sicchè, in base alla $\left(6_{79}\right)$, si vede che l'invariante di ZEUTHEN-SegRe di uno spazio proiettivo di dimensione $v$ vale $(-1)^{v-1}(v-1)$. 
rogliamo mostrare ch' essa è l' inversa della precedente, il che (nn. 7, 84) equivale a dire che, posto

$$
\psi_{v, k}=\sum_{i=0}^{k}(-1)^{i}\left(\begin{array}{c}
v+1 \\
i
\end{array}\right)\left(\begin{array}{c}
v+k-i \\
k-i
\end{array}\right)
$$

risulta

$$
\psi_{v, k}=0 \text { per } k>0
$$

A tal nopo osserviamo che la (6) porge:

$$
\begin{gathered}
\psi_{v, 0}=1 \quad \text { se } \quad v>0, \quad \psi_{0, k}=0 \quad \text { se } k>0, \\
\psi_{v, k}-\psi_{v-1, k}=\psi_{v, k-1}-\psi_{v-1, k-1} .
\end{gathered}
$$

Per indazione rispetto a $k$ ne consegue che, se $k>0, v>0$, risulta $\psi_{v, k}-\psi_{v-1, k}=0$; e da qui si deduce tosto la $(7)$, procedendo per induzione rispetto a $v$.

86. Avuto riguardo alle $\left(1_{85}\right),\left(3_{84}\right)$, per stabilire la $\left(2_{84}\right)$ basta mostrare che - per $i=1,2, \ldots, v$ - valgono le:

$$
[v]_{i}^{*}=(-1)^{i}\left(\begin{array}{c}
v+1 \\
i
\end{array}\right) \cdot[v-i]
$$

relazioni, queste, che, avuto riguardo a ciò che sì è detto nel n. 85 circa le $\left(5_{8 \overline{3}}\right)$, equivalgono alle

$$
[\tilde{v}]_{i}^{*}=\left(\begin{array}{c}
v+i \\
i
\end{array}\right) \cdot[v-i] .
$$

A tal uopo, procederemo per induzione completa rispetto alla differenza $v-i$. Quando questa differenza è zero, la (1) diventa

$$
(-1)^{v}[v]_{v}^{*}=(v+1) \cdot[0]
$$

e quindi esprime che il primo membro della (3) è un gruppo di $v+1$ punti di $[v]$. Ora, a norma del n. 66, il numero dei punti di tale gruppo uguaglia il grado della varietà diagonale sul prodotto $[v] \times[v]$. D'altro canto, questa varieta diagonale appartiene ad un sistema continuo di varietà $v$-dimensionali di quel prodotto, rappresentative delle omografie di $[v]$ in sè; e poichè la generica di queste nmografie ammette $v+1$ punti uniti (semplici e distinti), ne consegue che quel grado vale proprio $v+1$, cio che dimostra la (3).

Supponiamo ora che la differenza $v-i$ abbia un valore positivo fonde sarà $v \geq 2$; e - ragionando per induzione completa - ammettiamo di aver già stabilita la (1) per tutti $\mathrm{i}$ valori di $v$ ed $i$ in corrispondenza ai quali la differenza $v-i$ assume un valore inferiore al suddetto. Sarà dunque in particolare:

$$
\begin{aligned}
{[v-1]_{j}^{*} } & =(-1)^{j}\left(\begin{array}{l}
v \\
j
\end{array}\right) \cdot[v-j-1] & \text { per } j=0,1, \ldots, i \\
{[v]_{j}^{*} } & =(-1)^{j}\left(\begin{array}{c}
v+1 \\
j
\end{array}\right) \cdot[v-j] & \text { per } j=0,1, \ldots, i-1 .
\end{aligned}
$$


Avato riguardo a cio che si è osservato nel primo capoverso del presente numero, e tenuto conto del n. 7 , si ha che le (5) implicano le

$$
\left[\tilde{v}_{j j}^{*}=\left(\begin{array}{c}
v+j \\
j
\end{array}\right) \cdot[v-j] \quad \text { per } j=0,1, \ldots, i-1\right.
$$

e che, in forza delle (5) o (6), le (1) e (2) sono fra loro equivalenti. Denotato quindi con $\lambda$ l'ordine della $[\tilde{v}]_{i}^{*}$, posto ciod:

$$
[\tilde{v}]_{i}^{*}=\lambda \cdot[v-i]
$$

per dimostrare la (2), e quindi la (1), basta provare che è

$$
\lambda=\left(\begin{array}{c}
v+i \\
i
\end{array}\right) \text {. }
$$

All' uopo rileviano anzitutto che, in forza della $\left(1_{14}\right)$, la successione covariante d'immersione di un iperpiano $[v-1]$ di $[v]$ in questo spazio è quella costituita dalle varieta

$$
(-1)^{k} \cdot[v-k-1] \quad(k=0,1,2, \ldots) .
$$

D'altro canto, in forza della $\left(11_{67}\right)$, l'alternante di tale successione equivale alla

sicchè dev' essere

$$
\left\{[v-1]^{*}\right\} \cdot\left\{[\tilde{v}]^{*}\right\}
$$

$$
\sum_{j=0}^{k}[v-1]_{k-j}^{*}[v]_{j}^{*}=[v-k-1] \quad(k=0,1,2, . .)
$$

Se $k<i$, possiamo esprimere le varieta canoniche che compaiono nella (9) mediante le (4), (6), il che conduce all'identità algebrica

$$
\sum_{j=0}^{k}(-1)^{k-j}\left(\begin{array}{c}
v \\
k-j
\end{array}\right)\left(\begin{array}{c}
v+j \\
j
\end{array}\right)=1
$$

questa può venire stabilita direttamente senza difficolta $\left({ }^{32}\right)$, e vale anche naturalmente per $k \geq i$. Se ora facciamo nella (9) $k=i$, ed esprimiamo le varietà canoniche che compaiono nella relazione risultante mediante le (4), (6) e (7), otteniamo:

$$
\sum_{j=0}^{i-1}(-1)^{-j}\left(\begin{array}{c}
v \\
i-j
\end{array}\right)\left(\begin{array}{c}
v+j \\
j
\end{array}\right)+\lambda=1 .
$$

Se quindi confrontiamo quest' uguaglianza con quella fornita per $k=i$ dalla (10), ne deduciamo la (8); e cio completa la dimostrazione di quanto asse: rito nel n. 84 .

(32) All' uopo. denotato il primo membro della (10) con $\left(_{r, k}\right.$, si osservi dhe e. $m_{v, 0}=1$ e che, per $k>0$, risulta:

$$
\phi_{v, k}=\omega_{y, k}-\omega_{v, k-1},
$$

dove $\psi_{y, k}$ si definisca con la $\left(6_{85}\right)$. Ma allora la $\left(7_{85}\right)$ prova che

onde segue la (10).

$$
\left(w_{v, k}=\omega_{n, k-1}=\omega_{p, k-2}=\ldots=\omega_{v, 0}=1\right. \text {. }
$$




\section{\& III. - Identità fra la nuova e la vecchia definizione delle varietà canoniche.}

87. Nel presente paragrafo, estenderemo a varieta qualunque il risultato enunciato nel n. 84 per gli spazi proiettivi. A tal nopo, sarà opportuno modi. ficare leggermente la notazione delle varietà canoniche introdotta nel n. 79, denotando ora con ${ }^{*} W_{i}$ nna varietà canonica di $W$ di dimensione $w-i$, intesa nel senso classico; assumiamo cioè:

$$
{ }^{*} W_{i}=X^{w-i}(W)
$$

Si tratterà quindi di dimostrare che

Per ogni variet⿳亠㐅े irriducibile e non singolare $W$, le due successioni cano. niche $\left\{W^{*}\right\}$ e $\left\{{ }^{*} W\right\}$ coincidono.

Si noti che questo risultato è già acquisito nel caso che $W$ sia un qualunque spazio proiettivo, in base al paragrafo precedente.

85. Incominciamo col far vedere che, analogamente alla $\left(11_{67}\right)$,

Se $P$ denota una qualunque sottovarietà $d i \nabla$ ( $P$ e $V$ essendo virtual. mente prive di punti multipli), la successione covariante d'immersione di $P$ in $V$ si esprime in funzione delle successioni canoniche classiche di $P$ e di $V$ mediante la:

$$
\left\{P_{V}\right\}=(\{* \bar{P}\} \cdot\{* \tilde{\bar{V}}\})_{V} .
$$

La formula (1) da stabilire equivale chiaramente alla

$$
\{* P\}=\left(\left\{\bar{P}_{V}\right\} \cdot\{* V\}\right)_{V} ;
$$

e questa non è che un modo compatto di scrivere le:

$$
{ }^{*} P_{i}=\sum_{j=0}^{i}(-1) j P_{V, j} \cdot{ }^{*} V_{i-j} \quad(i=0,1,2, \ldots) .
$$

Si noti poi ehe, in forza delle $\left(5_{13}\right) \cdot\left(2_{79}\right)$, la (3) certamente sussiste per $i=0$.

Ineominciamo dal caso in cui $P$ sia un' i persuperficie di $V$, e cioè supponiamo risulti $v-p=1$; allora vale la $\left(1_{14}\right)$, onde la (3) riducesi alla

$$
* P_{i}=\sum_{j=0}^{i} P^{[j+1]} \cdot * V_{i-j} \text {. }
$$

Questa essendo orviamente soddisfatta per $i=0$, potremo supporre $i \geq 1$ e procedere per induzione rispetto ad $i$, ammettendo quindi che sia

$$
{ }^{*} P_{i-1}=\sum_{j=1}^{i} P^{[j]} \cdot{ }^{*} \nabla_{i-j} \text {. }
$$


In base al teorema dell' aggiunzione generalizzato $\left({ }^{33}\right)$, si ha inoltre:

$$
{ }^{*} P_{i}=P \cdot\left({ }^{*} P_{i-1}+{ }^{*} V_{i}\right) \text {. }
$$

Basta così eliminare ${ }^{*} P_{i-1}$ fra le ultime due relazioni, per dedurne la (4). Passiamo al caso in cui la differenza $v-p$ abbia un valore superiore all'unita. Procederemo allora per induzione rispetto a tale differenza, ammettendo il teorema per varietà le cui dimensioni abbiano una tal differenza inferiore a $v-p$. Scelta in $\nabla$ una sottovarietà irriducibile $M$ (virtualmente priva di punti multipli) che passi per $P$, possiamo quindi applicare la (1) a $P \subset M$ ed a $M \subset V$, ciò che fornisce le

$$
\left.\mid P_{M}\right\}=\left(\{* \bar{P} \mid \cdot\{* \tilde{\bar{M}}\})_{M}, \quad\left|M_{V}\right|=(\mid * \bar{M}\} \cdot|* \tilde{\bar{V}}|\right)_{V}
$$

D'altro canto, in virtù del teorema dell' appartenenza (n. 18), vale la $\left(3_{18}\right)$. Esprimendo in quest' ultima relazione $\left.\mid P_{M}\right\},\left|M_{V}\right|$ mediante le (5) ed applicando la $\left(5_{8}\right)$ otteniamo:

$$
\begin{aligned}
\left|P_{V}\right| & \left.=(|* \bar{P}| \cdot|* \tilde{\bar{M}}|)_{M} \cdot(|* \bar{M}| \cdot|* \tilde{\bar{V}}|)_{V}\right)_{M}= \\
& =\left(\left.\left(1^{*} \bar{P}|\cdot| * \tilde{\bar{M}}|\cdot| * \bar{M} \mid\right)_{M} \cdot\right|^{*} \overline{\bar{V}} \mid\right)_{V}= \\
& =\left(1^{*} \bar{P} \mid \cdot\{* \tilde{\bar{V}} \mid)_{V} .\right.
\end{aligned}
$$

donde segue la (1).

89. Riferiamoci ora ad una qualunque varieta $P$, irriducibile e non singolare, e denotiamo con $V$ uno spazio lineare che la contenga. In virtù del n. 84 , si ha conseguentemente

$$
\left|V^{*}\right|=1^{*} V \mid
$$

inoltre, in forza dei nn. 67,88 , sussistono le $\left(1_{67}\right),\left(2_{88}\right)$. Basta quindi confrontare queste due relazioni fra loro, tenendo presente la (1), per vedere che $\theta$ :

$$
\left|P^{*}\right|={ }^{*} P \mid \text {. }
$$

E oiò dimostra il teorema del n. 87.

90. Tenuto conto del n. 66 , si vede subito come il risultato stabilito nei nn. 87.89 fornisca in particolare nuove dimostrazioni per il principio di corrispondenza per le corrispondenze a valenza sopra una curva algebrica, assieme al relativo significato funzionale, nonchè per il teorema (ivi citato) che assegna il significato topologico della serie di Severi di una superficie. Vale infatti il primo teorema del n. 66, ove $\mathrm{i}$ gruppi canonici di $V$ possono ormai venir intesi nel senso classico; in virtù del n. 79 , il numero deí punti di tali gruppi è quindi quello fornito dalla $\left(6_{79}\right)$.

( ${ }^{33}$ ) Cfr. Eger [3], n. 22, Todd [7], n. 8 e Todd [8], n. 33. 
Basta ora combinare quest' ultimo risultato con la ben nota proprieta topologica $\left({ }^{34}\right)$ secondo cui l' indice di $\mathrm{Kronecker}$ della varietà diagonale di $\nabla \times V$ con se stessa sopra $\nabla \times V$ (e cioè il grado di tale varietà diagonale in $V \times \nabla$ ) uguaglia la caratteristica di Eulero-Poincaré (della riemanniana, a $2 v$ dimensioni) di $V$, per ottenere il teorema di Alexanner [1], affermante che :

La caratteristica di Eulero-Poincaré di una varietà algebrica irriducibile non singolare $V$, avente la dimensione $v$ e l'invariante di Zeuthen-Segre $I_{w}$, vale $(-1)^{v} I_{v}+2 v$. In altri termini, sussiste l' uguaglianza

$$
\left.D_{i v}=I_{v}+2(-1)^{v}(v-1)+2 \sum_{i=1}^{v-1}-1\right)^{v-i-i} R_{i},
$$

dove $R_{i}$ (per $i=1,2, \ldots, v$ ) denota $i l$ numero di Betti di dimensione $i$ relativo alla riemanniana ( $a 2 v$ dimensioni) di $V$.

La (1) mostra che $R_{v}$ ed $I_{v}$ hanno in ogni caso la stessa parità. E. poichè, notoriamente $\left({ }^{3 i}\right), R_{v}$ risulta pari se $v$ è dispari, cosı (ofr. B. SEgre [2]):

$L$ 'invariante di Zeuthen-Segre e l'ordine della serie di equivalenza cano. nica di ogni varietà algebrica di dimensione dispari, sono sempre numeri pari.

\section{§ IV. - I sistemi canonici sulle intersezioni complete.}

91. Detti $V=[v]$ uno spazio proiettivo di dimensione $v$, ed $A=[v-1]$ un suo iperpiano, in virtù dei nn. 84,86 risulta:

$$
V_{i}^{*}=(-1)^{i}\left(\begin{array}{c}
v+1 \\
i
\end{array}\right) A^{[i]}, \quad \quad \text { (2) } \quad \tilde{V}_{i}^{*}=\left(\begin{array}{c}
v+i \\
i
\end{array}\right) A^{[i]}
$$

$$
A_{i}^{*}=(-1)^{i}\left(\begin{array}{l}
v \\
i
\end{array}\right) A^{[i+1]} \text {. }
$$

D'altro canto, se $C$ denota una forma di $V$, d'ordine $c$, si ha

ed inoltre la $\left(2_{70}\right)$ porge :

$$
O=c A
$$

$$
C_{i}^{*}=\sum_{j=0}^{i} C^{[j+1]} \nabla_{i-j}^{*}
$$

Da qui, avuto riguardo alle (1), (4), consegue che əे

$$
C_{i}^{*}=\gamma(i) \cdot A^{[i+1]}
$$

dove $\gamma(i)$, e ciò̀ l'ordine della varietà canonica d'indice $i$ di una forma $C$ d' ordine $c$ in $[v]$, ̀̀ dato da

$$
\gamma(i)=\sum_{j=0}^{i}(-1)^{i-j}\left(\begin{array}{c}
v+1 \\
i-j
\end{array}\right) c^{j+1} \quad(i=0,1, \ldots, v-1)
$$

(34) Cfr. per esempio Lefschetz [1], p. 272.

(35) Ved. ad esempio Lefscherz [1], p. 218. 
Allo stesso risultato si può dare forma un po' diversa, ricorrendo al n. 73 . In virtù delle $(4),\left(6_{25}\right)$, se si considera la funzione razionale di $A$ :

$$
f(A)=\frac{c A+2\left(\begin{array}{l}
c \\
2
\end{array}\right) A^{[2]}+3\left(\begin{array}{l}
c \\
3
\end{array}\right) A^{[3]}+\ldots+c A^{[c]}}{1-\left(\begin{array}{l}
c \\
2
\end{array}\right) A^{[2]}-2\left(\begin{array}{l}
c \\
3
\end{array}\right) A^{[?]}-\ldots-(c-1) A^{[c]}},
$$

con le notazioni del n. 73 risulta

$$
\left\{C^{*}\right\}=\left\{f(A)^{*}\right\}
$$

e ciò significa che, se

$$
f(A)=\sum_{k} c_{k} A^{[k]}
$$

è lo sviluppo di $f(A)$ in serie di potenze di $A$, risulta:

$$
C_{i}^{*}=\sum_{k} c_{k}\left(A^{[k]}\right)_{i-k+1}^{*}=\sum_{k}(-1)^{i-k+1}\left(\begin{array}{l}
v-k+1) \\
i-k+1
\end{array} c_{k} \cdot A^{[i+1]}\right.
$$

Il confronto con la (6) porge quindi

$$
\gamma(i)=\sum_{k}(-1)^{i-k+1}\left(\begin{array}{c}
v-k+1 \\
i-k+1
\end{array}\right) c_{k}
$$

scrivendo che quest'espressione coincide con quella fornita dalla (7) si ottengono delle notevoli identità algebriche, che potrebbero nataralmente anche venir verificate direttamente.

Notiamo che, in base alle $\left(6_{79}\right),(7)$, risulta in particolare che la generica forma d'ordine $c$ di $[v]$ ha l'invariante di Zeuthen-Segre:

$$
I=\gamma(v-1)+(-1)^{v} 2(v-1)=(-1)^{v}\left(v+\frac{1}{c}-3\right)+\frac{1}{c}(c-1)^{v+1} .
$$

92 Se $M, N, \ldots, P$ denotano $s(\geq 2)$ varietà di $V$, che si incontrino regolarmente lungo la varietà

$$
R=(M N \ldots P)_{V},
$$

di dimensione $r=m+n+\ldots+p-(s-1) v>0$, per determinare la successione canonica di quest'ultima basta applicare la formula $\left(3_{72}\right)$. In tale formula compare la successione $\left\{\tilde{V}^{*}\right\}^{[s-1]}$, fornita dalla $\left(2_{91}\right)$ nell'ipotesi che sia $V=[v]$. Più precisamente, avuto riguardo alla $\left(2_{91}\right)$ ed al n. 6 , si ha allora:

$$
\left.\tilde{V}^{*}\right\}_{i}^{[s-1]} \ldots \Sigma\left(\begin{array}{c}
v+i_{1} \\
i_{1}
\end{array}\right)\left(\begin{array}{c}
v+i_{2} \\
i_{2}
\end{array}\right) \ldots\left(\begin{array}{c}
v+i_{s-1} \\
i_{s-1}
\end{array}\right) \cdot A^{[i]}
$$

ove la somma dev'essere estesa a tutte le $(s-1)-$ ple $i_{1}, i_{2}, \ldots, i_{s-1}$ soddisfacenti alle

$$
i_{1}+i_{2}+\ldots+i_{s-1}=i, \quad i_{1} \geq 0, i_{2} \geq 0, \ldots, i_{s-1} \geq 0
$$


Risulta pertanto:

$$
\begin{gathered}
\left|\tilde{V}^{*}\right|_{0}^{[s-1]}=V, \quad\left\{\left.\tilde{V}^{*}\right|_{1} ^{[s-1]}=(s-1)(v+1) \cdot A,\right. \\
\left|\tilde{V}_{2}^{*}\right|_{2}^{[s-1]}=\left[(s-1)\left(\begin{array}{c}
v+2 \\
2
\end{array}\right)+\left(\begin{array}{c}
s-1 \\
2
\end{array}\right)(v+1)^{*}\right] \cdot A^{[2]}, \quad \text { ecc. }
\end{gathered}
$$

I calcoli possono venir condotti in modo più semplice, osservando che la serie di potenze formali coordinata (n. 6) alla successione $\left(1_{91}\right)$ vale:

$$
\left[\left|V^{*}\right|, x\right]=(1-A x)^{v+1} \text {. }
$$

Pertanto, la serie di potenze coordinata alla $\left\{\check{V}^{*}\left\{^{[s-1]}\right.\right.$ vale:

$$
\left[1 \tilde{V}^{*}\{[s-1], x]=(1-A x)^{-(s-1)(v+1)}\right. \text {; }
$$

e da qui, sviluppando il secondo membro con la serie binomiale, si ricavano altre espressioni per le $\left|\tilde{V}^{*}\right|_{i}^{[s-1]}$.

Rileviamo infine che, in base alle $(1),\left(5_{91}\right)$, la serie di potenze coordinata alla successione canonica $\left\{C^{*}\right\}$, dove $C$ denoti una qualunque ipersuperficie $\left(4_{91}\right)$ di $[v]$, vale:

$$
\left[\left\{C^{*}\right\}, x\right]=C(1-A x)^{v+1}(1-c A x)^{-1} \text {. }
$$

Questa relazione porge - per le funzioni $\gamma(i)$ definite nel n. 91 - espressioni diverse dalle (ma equivalenti alle) $\left(7_{91}\right),\left(8_{91}\right)$.

93. Le considerazioni del numero precedente si semplificano, nel caso particolare in eui oi si riferisca ad una varieta

$$
P=\left(A^{\mathrm{i}} A^{2} \ldots A^{s}\right)_{V},
$$

di dimensione $p=v-s>0$, che sia l'intersezione completa di $s$ forme $A^{1}, A^{2}, \ldots, A^{s} d i \quad V=[v]$. In tale ipotesi valgono le $\left(1_{17}\right)$; sicchè, usufruendo delle $\left(1_{67}\right),\left(1_{92}\right)$, risulta che :

La serie di potenze formali coordinata alla successione canonica della varietà (1) suddetta, vale

$$
\begin{aligned}
{\left.\left[i P^{*}\right\}, x\right] } & =P(1-A x)^{v+1} \prod_{h=1}^{s}\left(1-A^{h} x\right)^{-1}= \\
& =P(1-A x)^{v+1} \prod_{h=1}^{s}\left(1-a_{h} A x\right)^{-1},
\end{aligned}
$$

denotando con $A$ un iperpiano di $V$ e con $a_{h} l$ l'ordine di $A^{h}$.

Questa proposizione $\left({ }^{36}\right)$, fornisce senz'altro le varietà canoniche di $P$ delle diverse dimensioni. Si ottiene cosi ad esempio subito che:

Le ipersuperficie canoniche della (1) sono quelle segate su essa dalle forme $d i V=[v]$ d' ordine $a_{1}+a_{2}+\ldots+a_{s}-v-1\left({ }^{37}\right)$.

(36) Includente un risultato di EGer [3], n. 31.

(37) Per una dimostrazione elementare di questa proposizione nel caso particolare che $P$ sia una superficie $(p=2)$, cfr. Godeaux [1]. 
Più in generale, per le $P_{i}^{*}$ vale il seguente risultato. Denotiamo con $\alpha_{i}$ la somma di tutti $i$ prodotti delle $a_{1}, a_{2}, \ldots, a_{s}$ prese con ripetizione ad $i$ ad $i$. Allora la (2) mostra che:

Le varietà canoniche $P_{i}^{*}$ d'indice $i$ della varietà $P$, definita dalla (1), vengono ivi segate da varietà di $V$ aventi dimensione $v-i$, il cui ordine risulta un polinomio di grado $i$ nelle $a_{1}, a_{2}, \ldots, a_{s}$, avente la somma $\alpha_{i}$ sud. detta come parte omogenea di grado $i$.

94 Riferendoci ad una qualunque varietà $P$ di dimensione $p$, che sia irriducibile e non singolare, denotiamo con $i_{1}, i_{2}, \ldots, i_{h}$ una partizione di $p$, ossia un qualsiasi insieme di un certo numero $h$ di numeri naturali $(1 \leq h \leq p)$, considerati a prescindere dall' ordine, che soddisfino alla

Allora è chiaro che

$$
i_{1}+i_{2}+\ldots+i_{h}=p
$$

$$
\left(P_{i_{1}}^{*} P_{i_{2}}^{*} \ldots P_{i_{h}}^{*}\right)_{P}
$$

risulta un gruppo di punti, definente su $P$ una serie di equivalenza invariante; pertanto il numero dei punti di tale gruppo, che denoteremo col simbolo

$$
\left[i_{1} i_{2} \ldots i_{h}\right]_{P},
$$

sarà un intero $(0)$ invariante di fronte alle trasformazioni birazionali regolari di $P$.

Detto $\pi(p)$ il numero delle partizioni distinte di $p$, talchè si ha

$\pi(1)=1, \quad \pi(2)=2, \quad \pi(3)=3, \quad \pi(4)=5, \quad \pi(5)=7, \quad \pi(6)=11, \quad \pi(7)=15 \ldots$, si ottengono su $P$ in tal guisa $\pi(p)$ serie di equivalenza (1) invarianti, e quindi altrettanti invarianti birazionali (2) di $P$. Ci proponiamo di dimostrare il seguente

Teorema. - Tanto le $\pi(p)$ serie di equivalenza invarianti $(1)$, quanto $i \pi(p)$ invarianti birazionali (2), risultano fra loro linearmente indipendenti,

con eiò intendendo significare che tanto una relazione del tipo

$$
\sum_{i} c_{i_{1} i_{2} \ldots i_{h}}\left(P_{i_{1}}^{*} P_{i_{2}}^{*} \ldots P_{i_{h}}^{*}\right)_{P}=0
$$

quanto una relazione del tipo

$$
\sum_{i} c_{i_{1} i_{2} \ldots i_{h}}\left[i_{1} i_{2} \ldots i_{h}\right]_{P}=0
$$

dove $c_{i_{1} i_{2} \ldots i_{h}}$ sia un intero dipendente eventualmente dalla partizione $i_{1}, i_{2}, \ldots, i_{n}$ ma non da $P$, può sussistere soltanto per valori tutti nulli delle $c$.

Poichè la (3) implica la (4), così manifestamente basterà stabilire la seconda parte del teorema. Supponiamo dunque che valga la (4) per ogni $P$; 
ed applichiamo in particolare la (4) alla varietà $P$ definita dalla $\left(1_{93}\right)$, per la quale conserviamo le notazioni del $\mathbf{n} .93$, facendo in più l'ipotesi che sia

$$
s \geq p \quad \text { (e ciò̀ } v \geq 2 p \text { ). }
$$

In base al risultato finale del $\mathrm{n}$. 93, il primo membro della (4) ò un poli. nomio di grado $s+p=v$ nelle $a_{1}, a_{2}, \ldots, a_{s}$, in quanto i coefficienti $c$ non possono dipendere dalle $a$, il quale contiene a fattore il prodotto $a_{1} a_{2} \ldots a_{s}$. Soppresso tale fattore ed uguaglia to a zero la parte omogenea di grado mas. simo nelle $a$ che cosi si ottiene, si giunge alla relazione omogenea di grado $p$ nelle $a$ :

$$
\sum_{i} c_{i_{1} i_{2} \ldots i_{h}} \alpha_{i_{1}} \alpha_{i_{2}} \ldots \alpha_{i_{h}}=0
$$

la quale - nelle nostre ipotesi - dev'essere identicamente soddi. sfatta.

Si osservi ora che, in virtù della (5), le $\alpha_{1}, \alpha_{2}, \ldots, \alpha_{p}$ sono $p$ funzioni delle $a_{1}, a_{2}, \ldots, a_{s}$ fra loro algebricamente indipendenti. Ne consegue che la (6) implica l'annullarsi di tutte le $c$, onde l'asserto $\left({ }^{38}\right)$.

95. Su di una varietà $P$ immersa in un'altra varietà $V$ (non necessariamente lineare) vi è luogo a considerare la successione canonica $\left|P^{*}\right|$, assieme alla successione covariante d'immersione $\left\{P_{V} \mid\right.$ (n. 13); ebbene, in virtù della $\left(11_{67}\right)$ e del numero precedente, queste due successioni sono f $\mathrm{ra}$ loro indipendenti. Mediante le intersezioni delle varietà di tali successioni si puo determinare su $P$ un certo numero, $\chi(p)$, di serie di equivalenza covarianti (o, in particolare, invarianti) fra loro linearmente indipendenti. Più precisamente, in corrispondenza ad ogni partizione $i_{1}, i_{2}, \ldots, i_{n}$ del numero $p$ si ottengono diverse serie di equivalenza siffatte, date dalla $\left(1_{\vartheta_{j}}\right)$ e dalle serie di equivalenza che si deducono dalla $\left(1_{94}\right)$ scrivendo in questa espressione al posto di uno o più dei fattori $P_{i}^{*}$ il corrispondente $P_{V, i}$. Gli ordini delle $\chi(p)$ serie di equivalenza suddette constituiscono altrettanti covarianti (o, in particolare, invarianti) numerici d'immersione di $P$ in $V$, fra loro linearmente indipendenti.

È facile vedere che si ha:

$$
\chi(1)=2, \quad \chi(2)=5, \quad \chi(3)=10, \quad \chi(4)=20, \quad \chi(5)=36, \ldots\left(^{39}\right) .
$$

(38) Un'argomentazione del tipo di quella testè usata, trovasi già in B. SEGre [7].

( $\left.{ }^{39}\right)$ Il fatto che una superficio (entro ad una varieta a quattro dimensioni) ammette cinque serie d'equivalenza covarianti fra loro indipendenti, trovasi già stabilito in $\mathrm{B}$. StGRE [7]. 


\section{§ V. - Alcuni complementi ed esempi.}

96. Sia $P$ una varieta algebrica, inriducibile e non singolare, appartenente ad uno spazio proiettivo $V$; e supponiamo che le dimensioni $p, v$ di $P, V$ soddisfino alla disuguaglianza

$$
2 p<v .
$$

Denoteremo con $p_{0}$ l' ordine di $P$ e, più generalmente, con $p_{k}$ (per $k=1$. $2, \ldots, p)$ l'ordine della varietà covariante d'immersione di $P$ in $V$ d'indice $k, P_{V, k}$ (la quale ha dimensione $p-k$ ). L'ordine di $P$ verrà anche indicato col simbolo $\pi_{0}$, in quanto designeremo più generalmerte con $\pi_{k}$ la classe $k-\mathrm{ma}$ di $P$ (e cioè l'ordine della varietà $(p-k)$-dimensionale luogo dei punti di contatio dei $[p]$ tangenti a $P$ che si appoggiano ad un dato $[v-p+k-2]$ secondo un $[k-1]$ ). Ci proponiamo di dimostrare che:

I suddetti caratteri $p_{k}$ si esprimono in funzione delle classi $\pi_{k}$ con le formule

$$
p_{k}=(-1)^{k} \sum_{j=0}^{k}\left(\begin{array}{c}
v-p+k-1 \\
k-j
\end{array}\right) \pi_{j} \quad(k=0,1, \ldots, p) .
$$

A tal nopo, consideriamo $v$ ipersuperficie $A^{1}, A^{2}, \ldots, A^{v}$ generiche di $V$ passanti per $P$; esse si segano in un gruppo $Q$ di punti fornito dalla relazione $\left(4_{13}\right)$, la quale attualmente si sorive

$$
\left(A^{1} A^{z} \ldots A^{y}\right)_{V}-Q=\sum_{i=0}^{p} P_{V, i} V_{p-i}^{v}(A) .
$$

Il numero $q$ dei punti del grappo $Q$ è quindi dato da :

$$
q=a_{1} a_{2} \ldots a_{v}-\sum_{k=0}^{p} p_{k} s_{n-k}
$$

dove $a_{1}, a_{2}, \ldots, a_{v}$ denotino gli ordini delle $A^{4}, A^{*}, \ldots, A^{v}, s_{0}=1 \mathrm{e}$, per $h \geq 1, \quad s_{h}$ designi la somma dei prodotti delle $a$ prese ad $h$ ad $h$ senza ripetizione.

D'altro canto, se indichiamo con $s^{\prime}{ }_{h}$ l'espressione che si deduce dalla suddetta $s_{h}$ sostituendo in essa ciascuna $a_{i}$ con $a_{i}-1$, quel numero $q$ - nell'ipotesi ammessa che valga la (1) - può anche venir espresso con la formula $\left({ }^{+0}\right)$ :

$$
\begin{gathered}
q=a_{1} a_{2} \ldots a_{v}-\left[\pi_{0} s_{p}^{\prime}+\left(\pi_{0}-\pi_{1}\right) s_{p-1}^{\prime}+\ldots+\left(\pi_{0}-\pi_{1}+\ldots \mp \pi_{p-1}\right) s_{1}^{\prime}+\right. \\
\left.+\left(\pi_{0}-\pi_{1}+\ldots \pm \pi_{p}\right) s_{0}^{\prime}\right] .
\end{gathered}
$$

Osserviamo ora che, dalla definizione delle $s, s^{\prime}$, seguono le

$$
s_{h}^{\prime}=\sum_{i=0}^{h}(-1)^{i}\left(\begin{array}{c}
v-h+i \\
i
\end{array}\right) s_{n}{ }^{-} i
$$

(40) Cfr. Severt [1], n. 35. 
talchè la (4) può anche venire scritta nella forma :

$$
q=a_{1} a_{2} \ldots a_{v}-\sum_{j=0}^{p} \sum_{k=j .}^{p} \sum_{h=j}^{k}(-1)^{j+h+k}\left(\begin{array}{c}
v-p+k \\
k-h
\end{array}\right) s_{p-k} \pi_{j} .
$$

Si verifica facilmente l'identità

$$
\sum_{h=j}^{k}(-1)^{h}\left(\begin{array}{c}
v-p+k \\
k-h
\end{array}\right)=(-1)^{s}\left(\begin{array}{c}
v-p+k-1 \\
k-j
\end{array}\right),
$$

mediante la quale l'ultima relazione si riduce alla

$$
\begin{aligned}
q & =a_{1} a_{2} \ldots a_{v}-\sum_{j=0}^{p} \sum_{k=j}^{p}(-1)^{k}\left(\begin{array}{c}
v-p+k-1 \\
k-j
\end{array}\right) s_{p-k} \pi_{j}= \\
& =a_{1} a_{2} \ldots a_{v}-\sum_{k=0}^{p} \sum_{j=0}^{k}(-1)^{k}\left(\begin{array}{c}
v-p+k-1 \\
k-j
\end{array}\right) \pi_{j} s_{p-k} .
\end{aligned}
$$

Basta dunque sottrarre questa a membro a membro dalla (3), per dedurre la

$$
\sum_{k=0}^{p}\left[p_{k}-(-1)^{n} \sum_{j=0}^{k}\left(\begin{array}{c}
v-p+k-1 \\
k-j
\end{array}\right) \pi_{j} \mid s_{p k}=0 .\right.
$$

Ora, mentre le espressioni qui scritte entro parentesi quadre non dipendono dalle $a_{1}, a_{2}, \ldots, a_{v}$, le $s_{n}=1, s_{1}, \ldots, s_{p}$ sono $p$ funzioni delle $a$ fra loro funzionalmente indipendenti. Stante l'arbitrarietà delle $a$, ne segue che ciascuna di quelle espressioni deve annullarsi, ciò che fornisce appunto le (2).

Le relazioni $(2)$, così stabilite, dànno rispettivamente per $k=0,1, \ldots$ le

$$
p_{0}=\pi_{n}, \quad-p_{1}=(v-p) \pi_{0}+\pi_{1}, \ldots,
$$

la prima delle quali era evidente a priori. La seconda, nell'ipotesi che $P$ sia una curva $(p=1)$ di genere $\pi$, pud anche venir scritta nella forma

$$
-p_{1}=(v+1) \pi_{0}+2 \pi-2,
$$

essendo allora $\pi_{1}=2\left(\pi_{n}+-\pi-1\right)$ in virtù della formula di Riemann per il genere. $\dot{\mathrm{E}}$ chiaro come le (2), risolte rispetto alle $\pi_{j}$, forniscano le classi $\pi_{j}$ in funzione dei caratteri d'immersione $p_{k}$.

97. Applicheremo ciò che precede alla determinazione del genere $\pi$ della curva $P$ secondo cui si segano due varietà $M, N$ (di dimensioni $m, n$ ) gene. ricamente situate in $V=[v]$, quando sia

$$
m+n=v+1
$$

supponendo di conoscere gli ordini $m_{0}, n_{0}$ ed i generi sezionali $\mu, \vee$ delle $M, N$. All' uopo osserviamo che, in virtù del teorema del prodotto (n. 20), dalla $P=(M N)_{V}$ segue la

$$
P_{1}=M N_{1}+M_{1} N
$$


In base alla $\left(5_{96}\right)$, la varietà $P_{\ell}$ ha l'ordine

$$
-\left[(v+1) m_{0} n_{0}+2 \pi-2\right] \text {. }
$$

Del pari, la $\left(5_{96}\right)$ combinata col teorema della sezione (n. 19) fornisce per gli ordini delle $M_{1}, N_{1}$ rispettivamente i valori

$$
-\left[(n+1) m_{0}+2 \mu-2\right], \quad-\left[(m+1) n_{0}+2 \nu-2\right] .
$$

Basta quindi uguagliare gli ordini dei due membri della (2), nsufruendo della (1), per ricavare la formula richiesta $\left({ }^{41}\right)$ :

$$
\pi=\left(m_{0}-1\right)\left(n_{0}-1\right)+m_{0} v+n_{n} \mu .
$$

98. Riferendoci di nuovo alla varietà generale $P$ di cui al n. 96 , si voglia determinare il numero $c$ dei punti del gruppo intersezione residua di $v$ forme $A^{1}, A^{2}, \ldots, A^{v}$ che passino genericamente per $P$ con date molteplicita $k_{1}$, $k_{2}, \ldots, k_{v}$. Questo problema è stato trattato dal SEverI ([1], n. 38), nell'ipotesi che le molteplicita $k$ assumano soltanto il valore 1 od il valore 2 ; e tale Autore (in [1], n. 39) si arresta quindi di fronte al caso generale, rilevando che «le espressioni che (per esso) si otterrebbero sarebbero algebrica"mente assai complicate». Qui siamo in grado di dare $c$ in forma esplicita per valori arbitrari delle $k$, come conseguenza numerativa di una particolarizzazione dei risultati funzionali conseguiti nel n. 45 .

Più precisamente, con le notazioni del n. 96 , dalla $\left(1_{45}\right)$ si ricava senz'altro $\operatorname{che} \Theta$ è

$$
c=a_{1} a_{2} \ldots a_{v}-\sum_{l=0}^{p} p_{l} r_{p-l}
$$

dove $r_{p-l}$ denoti la somma dei $\left(\begin{array}{l}v \\ l\end{array}\right)$ prodotti del tipo

$$
k_{i_{1}} k_{i_{2}} \ldots k_{i_{l}} a_{j_{1}} a_{j_{2}} \ldots a_{j_{v-l}}
$$

ottenibili assumendo per $i_{1}, i_{z}, \ldots, i_{l}$ una combinazione di classe $l$ dei numeri $1,2, \ldots, v$ e per $j_{1}, j_{2}, \ldots, j_{v-l}$ la combinazione complementare.

Nella (1), volendo, si possono far comparire - in luogo dei caratteri $p_{l}-$ le classi $\pi_{j}$, servendosi delle $\left(2_{96}\right)$. Ed è poi facile verificare come il risultato cosi ottenuto collimi con quello di Severi, nel caso - già da lui studiato in cui parte delle $k$ abbiano il valore 1 e le altre assumano il valore 2.

99. Sulla varieta $P$ vi è anche luogo a considerare la successione canonica $\left\{P^{*}\right\}$ e l'inversa $\left\{\tilde{P}^{*}\right\}$ di questa; denoteremo rispettivamente con $p_{i}^{*}, \tilde{p}_{i}^{*}$ gli ordini delle relative varietà $P_{i}^{*}, \tilde{P}_{i}^{*}$ (sicchè sarà $\left.p_{0}^{*}=\tilde{p}_{0}^{*}=p_{0}\right)$.

(41) Cfr. Longhi [1], n. 2. 
Senza fare ora nessuna restrizione circa la dimensione $v$ dello spazio $V$ di appartenenza di $P$, possiamo applicare le $\left(1_{67}\right),\left(11_{67}\right)$ a $P$ e $V$, ottenendo così le equivalenze:

$$
P_{i}^{*}=\sum_{j=0}^{i}(-1)^{j} P_{V, j} V_{i-j}^{*}, \quad \tilde{\bar{P}}_{V, i}=\sum_{j=0}^{i} \tilde{P}_{j}^{*} V_{i-j}^{*} .
$$

Detti quindi $p_{i}, \tilde{p}_{i}$ gli ordini delle $P_{V, i}, \tilde{P}_{V, i}$, e tenuto conto del n. 86 , da qui si ricavano senz' altro le relazioni numerative

$$
p_{i}^{*}=(-1)^{i} \sum_{j=0}^{i}\left(\begin{array}{c}
v+1 \\
i-j
\end{array}\right) p_{j}, \quad \quad \text { (2) } \quad \tilde{p}_{i}=\sum_{j=0}^{i}(-1)^{j}\left(\begin{array}{c}
v+1 \\
i-j
\end{array}\right) \tilde{p}_{i}^{*}
$$

Queste possono venir risolte rispetto alle $p, p^{*}$, poggiando sulle proprietà delle fanzioni $\left(6_{85}\right)$ espresse dalle $\left(7_{85}\right),\left(8_{85}\right)$; in tal guisa si ricavano le:

$$
p_{i}=(-1)^{i} \sum_{j=0}^{i}\left(\begin{array}{c}
v+i-j \\
i-j
\end{array}\right) p_{j}^{*}, \quad \text { (4) } \quad \tilde{p}_{i}^{*}=\sum_{j=0}^{i}(-1)^{j}\left(\begin{array}{c}
v+i-j \\
i-j
\end{array}\right) \tilde{p}_{j}
$$

E inoltre chiaro, in base ai nn. 6, 7, che per $i>0$ valgono le identità:

$$
\sum_{j=0}^{i} p_{j} \tilde{p}_{i-j}=0, \quad \sum_{j=0}^{i} p_{j}^{*} \tilde{p}_{i-j}^{*}=0
$$

le quali risultano fra loro equivalenti in forza delle (1), (4) [o delle (2), (3)].

100. Sulla $P$, appartenente a $V=[v]$, considereremo le varietà $M_{i}, N_{i}$, di dimensione $p-i$, rispettivamente luoghi dei punti di $P$ relativamente ai quali lo spazio $[p]$ tangente a $P$ risulta appoggiato ad un dato $[v-p+i-2]$ secondo un $[i-1]$, oppure risulta appoggiato (generalmente in un punto) ad un dato $[v-p-i]$. La varietà $M_{i}$ è definita per $i=0,1, \ldots, p$, ed ha per ordine l'i-ma classe $\pi_{i}$ di $P$ (n. 96). La varietà $N_{i}$ è definita per $i=0,1, \ldots, p$ se $v \geq 2 p$, od altrimenti soltanto per $i=0,1, \ldots, v-p$; il suo ordine, $\omega_{i}$, ஓे quello che - con Severi [1] - chiamasi il ceto $i-\mathrm{mo}$ di $P$.

Detto $A$ un iperpiano di $[v]$, le suddette varietà $M, N$ si esprimono con le equivalenze $\left({ }^{42}\right)$ :

$$
M_{i}=\sum_{j=0}^{i}\left(\begin{array}{c}
p-i+j+1 \\
j
\end{array}\right) P_{i-j}^{*} A^{[j]}, \quad N_{i}=\sum_{j=0}^{i}\left(\begin{array}{c}
p+i \\
j
\end{array}\right) \tilde{P}_{i-j}^{*} A^{[j]}
$$

dalle quali si deducono le relazioni numerative:

$$
\pi_{i}=\sum_{j=0}^{i}\left(\begin{array}{c}
p-i+j+1 \\
j
\end{array}\right) p_{i-j}^{*}, \quad \text { (2) } \quad \omega_{i}=\sum_{j=0}^{i}(-1)^{i-j}\left(\begin{array}{c}
p+i \\
j
\end{array}\right) \tilde{p}_{i-j}^{*}
$$

(4:) Cfr. J. A. Topd [8], formule (311) e (3.12). 
e queste a loro rolta forniscono le:

(3) $\quad p_{i}^{*}=\sum_{j=0}^{i}(-1)^{j}\left(\begin{array}{c}p-i+j+1 \\ j\end{array}\right) \pi_{i-j}, \quad$ (4) $\quad \tilde{p}_{i}^{*}=\sum_{j=0}^{i}(-1)^{i-j}\left(\begin{array}{c}p+i \\ j\end{array}\right) \omega_{i-j}$.

Si ottengono immediatamente equazioni equivalenti alle $\left(2_{96}\right)$, eliminando le $p^{*}$ fra le $\left(3_{99}\right)$ e le $(3)$.

101. Applicheremo da ultimo le formule precedenti, per compiere qualche verifica numerativa dei risultati funzionali conseguiti nei nn. 48 , 50. Per semplicità algoritmica ci rifuriremo ad un caso estremanente particolare, supponendo (con le notazioni di tali numeri):

$$
V=[v], \quad p=v-2 \geq 2, \quad m=v-1 .
$$

Pertanto, $P$ sarc̀ una varietà di dimensione $p$ appartenente ad nno spazio di dimensione

$$
v=p+2,
$$

ed $M$ sarà un'ipersuperficie di questo spazio costretta a passare per $P$, l'ordine della quale verrà designato con $\mu$.

La varietà $P$ ammetterà allora un luogo $D$ di punti doppi impropri (n. 46), di dimensione

$$
d=v-4 \text {, }
$$

il eni ordine denoteremo con $d_{\theta}$. Inoltre $M$, fuori di $D$, avrà su $P$ un luogo $\Delta$ di punti doppi, di dimensione (n. 49)

$$
\delta=v-4
$$

il cni ordine sarà similmente indicato con $\delta_{v}$.

Dalle $\left(1_{48}\right),\left(2_{51}\right)$, avuto anche riguardo alla $\left(1_{14}\right)$ ed a ciò che s'è detto alla fine del n. 48 , si ricavano senzi altro le

$$
\begin{gathered}
d_{0}=\frac{1}{2} p_{0}^{2}-\frac{1}{2} \tilde{p}_{2}, \\
\delta_{0}=2 \tilde{p}_{2}-p_{0}^{2}-\tilde{p}_{1} \mu+p_{0} \mu^{2} .
\end{gathered}
$$

Ora le $\left(2_{100}\right)$, tenuto conto della (1), attualmente forniscono:

$$
\omega_{0}=p_{0}, \quad \omega_{1}=(v-1) p_{0}-\tilde{p}_{1}^{*}, \quad \omega_{2}=\left(\begin{array}{l}
v \\
2
\end{array}\right) p_{0}-\cdots \tilde{p}_{1}^{*}+\tilde{p}_{\mathfrak{z}}^{*},
$$

sicohe, in base anche alle $\left(2_{99}\right)$, risulta:

$$
\omega_{0}+\omega_{1}+\omega_{2}=\left(\begin{array}{c}
v+1 \\
2
\end{array}\right) p_{0}-(v+1) \tilde{p}_{1}^{*}+\tilde{p}_{2}^{*}=\tilde{p}_{2} .
$$


In virtù delle (4), (5), la (2) si riduce alla

$$
d_{0}=\frac{1}{2}\left(\omega_{0}^{2}+\omega_{0}+\omega_{1}+\omega_{2}\right)
$$

e ciò collima con una formula di Severi ([1], n. 3).

Dalle $\left(2_{99}\right),\left(4_{100}\right),(4)$ si ha inoltre:

$$
\begin{aligned}
\tilde{p}_{1}=(v+1) p_{0}-\tilde{p}_{1}^{*} & =(v+1) p_{0}-(v-1) \omega_{0}+\omega_{1}= \\
& =2 \omega_{9}+\omega_{1} .
\end{aligned}
$$

Basta quindi applicare le (4), (5), (6) per mettere la (3) sotto la forma

$$
\delta_{0}=(\mu-1)^{2} \omega_{0}-(\mu-1) \omega_{1}+\omega_{2}+\left(\omega_{1}+\omega_{2}\right)-\omega_{0}\left(\omega_{0}-1\right),
$$

d'accordo ancora con un risultato di SeverI ([1], n. 7).

Il lato numerativo dei risultati funzionali precedentemente ottenuti può naturalmente venir esplicitato senza sostanziali difficoltà in ogni caso, procedendo in modo consimile a quello seguito nei casi semplici ai quali ci siamo limitati - per controllo ed esempio - nel presente paragrafo $\left({ }^{43}\right)$.

\section{BIBLIOGRAFIA}

[1] G. ALBANESE, Formule fondamentali della geometria sopra una varietò algebrica, . Annali di Mat. ", (4) 4 (1927), 154-184.

[1] J. W. ALEXANDER, Sur les cycles des surfaces algébriques et sur une definition topologique de l' invariant de Zeuthen-Segre, "Rend. Acc. Naz. Lincei ", (5) 23 (1914) 2 , 55.62.

[1] J. W. Archbold, Multiple intersections on an algebraic $V_{4}$, "Proc London Math. Soc. ", (2) $47(1941), 101 \cdot 122$.

[1] C. C. H. BARKeR, Intersections and contacts of surfaces on a $V_{3}$, * Journ. London Math. Soc. », 26 (1951), 125-131.

[1] S. S. Chern, Characteristic classes of Hermitian Manifolds, "Ann. of Math. ", 47 (1946), 85-121.

[1] A. Comessatru, Sulla serie canonica duna superficie algebrica, "Rend. Acc. Naz. Lincei », (6) 16 (1932), 555.560.

[1] F. Conforto, Lo stato attuale della teoria dei sistemi d'equivalenza e delle corrispondenze algebriche tra varietò, "Atti Convegno Mat. * tenuto in Roma nel 1942, pp. 49.83.

[1] I. Eger, Determinazione del gruppo dei punti doppi acquisiti da una forma dell' $S_{2 k}$ passante per una data $V_{k}$, \& Boll. Un. Mat. Ital. ", (1) 15 (1936), 56-61.

[2] M. EGRR, Sur les systèmes canoniques d'une variété algébrique, "Comptes Rendus Ac. Sc. ", 204 (1937), 92-94 e 217-219.

[3] M. EGER, Les systèmes canoniques d'une variété algébrique à plusieurs dimensions (Thèse), «Ann. Ec. Norm. Sup. , (1943).

[1] F. ENRIQUES, Intorno ad alcune serie invarianti di gruppi di punti sopra una superficie algebrica, «Rend. Acc. Naz. Lincei », (6) 16 (1932), 533-540.

(43) Il contenuto di questa Memoria ha formato oggetto di un Corso di conferenze presso l' Università di Liegi, tenuto dall'autore nell'aprile 1953, nel quadro dell' accordo culturale italo-belga, e di una serie di lezioni presso l'Istituto Nazionale di Alta Matematica. 
[1] F. GAETA, Sui sistemi lineari appartenenti al prodotto di pì̀ varietà algebriche, *Ann. di Mat. ", (4) 33 (1952), 91-118.

[1] L. Godmaux, Sur les surfaces algébriques intersections complètes d'hypersurfaces, · Revista de Tucuman, 2 (1941), 211.216.

[1] W. V. D. HodqE, The characteristic classes on algebraic varieties, Proc. London Math. Soc. ", (3) 1 (1951), 138-151.

[1] W. V. D. Hodge-D. Pedow, Methods of algebraic geometry, vol. II, Cambridge, Univ. Press, 1952.

[1] S. Lewschetz, Topology, •Colloquium Pubbl. s, t. 12 (New York, 1930).

[2] S. Lewschetz, Algebraic topology, "Colloquium Pubbl.", t. 27 (New York, 1942).

[1] A. Longhr, Sulla intersezione di due o più varietà algebriche, "Comm. Math. Helv. ", $18(1.945-46), 45-51$.

[1] R. E. Mavpherson, Canonical systems on a redueible variety, "Proc. Cambridge Phil. Soc. ", 35 (1939), 389-393.

[1] B. Segre, Nuovi contributi alla Geometria sulle varietà algebriche, "Mem. Acc. d'Italia ", 5 (1934), 479-576.

[2] B. SEGRE, Intorno alla parità di alcuni caratteri di una varietà di dimensione dispari, - Boll. Un. Mat. Ital. ", (1) 13 (1934), 93.95.

[3] B. Segre, Quelques résultats nouveaux dans la géométrie sur une $V_{3}$ algébrique (Mémoire couronné), "Mem. Ac. Roy. Belgique s; (2) 14 (1936), 3.99.

[4] B. SEGRE, Un problema di geometria numerativa, *Boll. Un. Mat. Ital. ", (1) 15 (1986), $49-55$.

[5] B. SEgRE, On limits of algebraic varieties, in particular of their intersections and tangential forms, "Proc. London Math. Soe. ", (2) 47 (1942), 351-408.

[6] B. SEGRE, Sullo scioglimento delle singolarità delle varietò algebriche, Ann di Mat. , (4) 33 (1952), 5.48.

[7] B. SEGRE, Sulla serie caratteristica d'una superficie sopra una varietà algebrica a quattro dimensioni, "Rend. Acc. Naz. Lincei", (6) 17 (1933), 917.918.

[8] B. Segre, L'anneau d'équivalence sur une variété algébrique, "Comptes Rendus Ac. Sc. "; 234 (1952), 1663-5.

[9] B. SEGRE, Variétés covariantes d'immersion et variétés canoniques sur une variété algé. brique on topologique, "Comptes Rendns Ac. Sc. ", 234 (1952), 17B1.3.

[1] C. SEGRE, Intorno ad un carattere delle superficte e delle varietà superiori algebriche, "Atti Acc. Se. Torino », 31 (1896), 485̄501.

[1] F. SEVERI, Sulle intersezioni delle varietà algebriche e sopra i loro caratteri e singolarità proiettive, "Mem. Acc. Sc. Torino», (2) 52 (1902), 61.118, oppure Memorie scelte, vol. I (Bologna, Zuffi, 1950), 41-115.

[2] F. SrverI, Fondamenti per la geometria sulle varietà algebriche, a Rend. Circ. Mat. Pa. lermo», 28 (1909), 33-87.

[3] F. SeVeri, La serie canonica e la teoria delle sevie principali di gruppi di punti sopra una superficie algebrica, "Comm. Math. Helv.", (1932), 287.345.

[4] F. SEvERI, Un nuovo campo di vicerche nella geometria sopra una superficie e sopra una varietà algebrica, "Mem. Acc. d'Italia ", 3 (1932), 5-52.

[5] F. Severi, Contributi alla teoria delle serie e dei sistemi di equivalenza sulle varietì algebriche, "Mem. Acc. d'Italia ", 8 (1937), 387-410.

[6] F. SEVERT, Serie, sistemi d'equivalenza e corrispondenze algebriche sulle varietà algebriche, vol. I (a cura di F. Conforto e di E. Martinfilli), Roma, Cremonese, 1942.

[7] F. SeverI, Il punto di vista gruppale nei vari tipi di equivalenza sulle varietà algebriche, "Cornm. Math. Helv. ", 21 (1948), 189.224.

[8] F. Severr, Fondamenti per una teoria generale dei connessi, Acta Salmanticensia, 1950. 
[9] F. SEVERI, Fondamenti per la geometria sulle varietà algebriche (Seconda Memoria), - Ann. di Mat. », (4) 32 (1951), 1.81.

[10] F. Stever, Equivalenza d'una curva come gruppo virtuale parziale d'una serie d'equivalenza sopra una superficie, Scritti mat. offerti a L. Berzolari (Pavia, 1936), 77-84.

[11] F. SEVRT, Sulle molteplicità d'intersezione delle varietà algebriche ed analitiche e sopra una teori. geometrica dell'eliminazione, a Math. Zeitsch. », 52 (1950). 827-851.

[1] P. Thullen, Determinazione della serie di equivalenza individuata dal gruppo dei punti doppi impropri d'una superficie dell' $S_{4}$, e Rendic. del Circ. Mat. di Palermos, 59 (1935), 256-260.

[1] J. A. TODD, Some group-theoretic considerations in algebraic geometry, Ann of Math.", (2) 35 (1934), 702.704.

[2] J. A. Tood, The geometrical invariants of algebraic loci, "Proe. London Math. Soc.", (2) 43 (1937), 127-138.

[3] J. A. Tond, The arithmetical invariants of algebraic loci, "Proc. London Math. Soc. *, (2) 43 (1937), 190.225.

[4] J. A. Tond, Birational transformations with isolated fundamental points, "Proc. Edinburgh Math. Soc. *, (2) 2 (1987), 117.124.

[5] J. A. ToDD, Intersections of loci on an algebraic $V_{4}$, "Proc. Cambridge Phil. Soc. ", 33 (1987), 425-437.

[6] J. A. TodD, Birational transformations possessing fundamental curves, * Proc. Cumbridge Phil. Soc. „, 34 (19 8), 144-155.

7] J. A. ToDd, The geometrical invariants of algebraic loci (second paper), "Proc. London Math. Soc. ", (2) 45 (1939), 410.424.

[8] J. A. ToDD, Invariant and covariant systems on an algebraic variety, « Proc. London Math. Soc. ", (2) 46 (1940), 199.230.

[1] J. A. TODD - E. A. MAXWELL, Note on the invariants of the canonical systems of an algebraic variety, * Proc. Cambridge Phil. Soc.", 33 (1987), 438-443.

[1] A. WEIL, Foundations of algebraic geometry, "Colloquium Pubbl. ", t. 29 (New York, 1946).

[1] A. L. Yoxail, Note on a paper by J. A. Todd, "Proc. Cambridge Phil. Soc.", 35 (1939), 125-126.

[1] O. Za Ariski, The fundamental ideas of abstract algebraic geometry, *Proc. of the Internat. Congr. Cambridge", 1950, vol. II (Amer. Math. Soc., 1952), 77-89. 